\title{
A Study of the Effects of Strong Magnetic Fields on the Image Resolution of PET Scanners
}

\section{DISSERTATION}

\author{
Presented in Partial Fulfillment of the Requirements for \\ the Degree Doctor of Philosophy in the \\ Graduate School of The Ohio State University \\ By
}

Don J. Burdette, B.S.

$* * * * *$

The Ohio State University

2009

Dissertation Committee:

Approved by

Klaus Honscheid, Adviser

Harris Kagan

Thomas Humanic

Adviser

Terrence Walker

Graduate Program in

Physics 
(C) Copyright by

Don J. Burdette

2009 


\begin{abstract}
Very high resolution images can be achieved in small animal PET systems utilizing solid state silicon pad detectors. In such systems using detectors with sub-millimeter intrinsic resolutions, the range of the positron is the largest contribution to the image blur. The size of the positron range effect depends on the initial positron energy and hence the radioactive tracer used. For higher energy positron emitters, such as ${ }^{68} \mathrm{Ga}$ and ${ }^{94 m} \mathrm{Tc}$, the variation of the annihilation point dominates the spatial resolution. In this study two techniques are investigated to improve the image resolution of PET scanners limited by the range of the positron. One, the positron range can be reduced by embedding the PET field of view in a strong magnetic field. We have developed a silicon pad detector based PET instrument that can operate in strong magnetic fields with an image resolution of $0.7 \mathrm{~mm}$ FWHM to study this effect. Two, iterative reconstruction methods can be used to statistically correct for the range of the positron. Both strong magnetic fields and iterative reconstruction algorithms that statistically account for the positron range distribution are investigated in this work.
\end{abstract}




\section{ACKNOWLEDGMENTS}

One day, as a young child, I skipped school to avoid a spelling test. During that

day, as I acted out all sorts of ailments, I watched a television program that opened my eyes to the wonders of science. The narrator of this program, Carl Sagan, postulated the following question: what if the speed of light was $50 \mathrm{~km} / \mathrm{hr}$ and you went out on your motor bike for a tour of the country side? What would you find when you returned? If you are unfamiliar with the concepts of special relativity, as I was at the time, the answers will shock you. The concepts explained in this program are more than enough motivation to influence any young mind. Mine was no exception. Now, twenty-three years later, I have completed the requirements for a $\mathrm{Ph}$. D. in physics and am heavily reliant on my spell checker.

Although this particular day has a special place in my memory, I would not be where I am today without the love and support of my family and the guidance and encouragement of my mentors and peers. Specifically, I would like to thank my parents (Jeanne and John Burdette) and sisters (Christine and Jeanne-Marie Burdette) for their constant encouragement throughout my entire life. I would also like to thank Mr. Likovitch, my high school physics instructor, for teaching me not to burn bridges but to break them. I would like to thank Mrs. Ligner and Mrs. Manion for freeing me from shyness and opening the door to creativity and humor. I do not want to thank Mr. Grind. 
The greatest influence on my professional physics development is due to my graduate advisor Dr. Klaus Honscheid. I would not be where I am today without his guidance, patience, and knowledge. I could never have asked for a better research adviser. I would also like to thank Dr. Harris Kagan for everything that he has taught me. I would like to thank the CIMA collaboration for all of their help and support. In particular I would like to thank Neal Clinthorne for sharing his expertise on every aspect of my project. I would like to thank Sang-June Park, Andrej Studen, Gabriela Llosa, and Sam Huh for getting me started on my research. This project could not have been completed without the assistance of Bob Wells (for his excellent craftsmanship), Shane Smith (for his in depth knowledge of electronics), and Jim Burns (for his wire bonding and encapsulating favors). I would also like to thank Michael Knopp, Petra Scholbrock, Jonda Lesser, Amir Abduljalil, and Frankie Aguila from the Wright Center of Innovation for allowing me to use their 7 T MRI magnet while making me feel welcome at the same time (you threw the best pot-luck parties).

I would like to thank all of my friends that have helped me throughout graduate school: Iulian Hetel, Mike Boss, James Morris (thanks for the pens), Joe Regensberger, Lee Mosbacker, Dirk Hufnagel, Even Froderman, Aaron Sander, Greg Mack, Caitlin Molone, Jacob Editing and the entire pizza crew. I would also like to thank all of my Sharon Pennsylvania friends for making me feel welcome every time I come home to visit. I also extend my gratitude to my friends Bill, Frank, Louise, and Zoey for helping me relieve stress and never letting me get left behind.

Most importantly, I would like to thank Christina Kwapich for showing me that there is more to life than either physics or Star Trek can offer. 


\section{VITA}

\begin{tabular}{|c|c|}
\hline October 17, 1979 & Born in Sharon Pennsylvania \\
\hline June 1998 & $\begin{array}{l}\text { Graduated from Sharon High School, } \\
\text { Sharon PA }\end{array}$ \\
\hline June 2002 & $\begin{array}{l}\text { Graduated with a B.S. in Physics from } \\
\text { the Indiana University of Pennsylvania, } \\
\text { Indiana PA }\end{array}$ \\
\hline June2002 - Present & $\begin{array}{l}\text { Ph.D. Candidate, The Ohio State Uni- } \\
\text { versity, Columbus } \mathrm{OH}\end{array}$ \\
\hline
\end{tabular}

Major Field: Physics 


\section{TABLE OF CONTENTS}

Page

Abstract .......................... ii

Acknowledgments .......................... iii

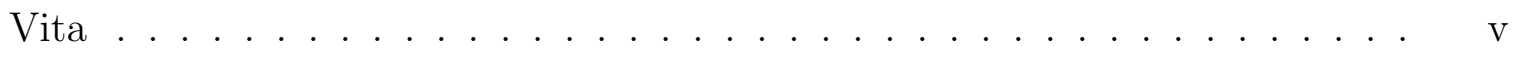

LIST OF TABLES $\quad \mathrm{x}$

LIST OF FIGURES Xii

Chapters:

1. An Introduction to Positron Emission Tomography 1

1.1 The Basics of the PET Principle . . . . . . . . . . . 1

1.2 PET Design Considerations . . . . . . . . . . . . . 5

1.2.1 Spatial resolution and sensitivity of the photon detectors . . 6

$1.2 .2 \quad$ Positron Range . . . . . . . . . . . . . . . . . . . . 9

1.2 .3 Doppler broadening . . . . . . . . . . . . . . . . . 10

1.2 .4 Timing resolution $\ldots \ldots \ldots \ldots \ldots \ldots$

$1.2 .5 \quad$ System Dead Time . . . . . . . . . . . . . . . . 13 
1.2 .6 Sensitivity Considerations _ . . . . . . . . . . . 13

1.3 Applications of PET Technology . . . . . . . . . . . . . . . . . . 14

1.4 Thesis Overview: Techniques to reduce the image blur due to the positron range . . . . . . . . . . . . . . . 16

\section{PET Physics and Simulations 19}

2.1 Overview . . . . . . . . . . . . . . . . . . . . . . . . . . . . 19

2.2 The Electron Gamma Shower Simulation Package (EGS4) _ . . . . 19

2.3 Simulating Positron Energy Spectrum . . . . . . . . . . . . . . . 24

2.4 Positron Penetration Through Matter . . . . . . . . . . . . 27

2.4.1 Molière's multiple scattering theory . . . . . . . . . . . . . 29

2.4 .2 Bhabha Scattering . . . . . . . . . . . . . . . 31

2.4.3 Inelastic energy losses due to soft collisions . . . . . . . . . . 33

2.5 The effect of magnetic fields on charged particles . . . . . . . . 38

2.6 Positron-electron annihilation and Doppler Broadening . . . . . . . 40

2.7 Results of Positron Range Simulations _. . . . . . . . . . . . . 42

2.8 Photon Interactions with matter . . . . . . . . . . . . 55

2.8 .1 Compton Scattering _ . . . . . . . . . . . . . 55

2.8.2 Coherent Rayleigh Scattering _ . . . . . . . . . . . . 59

2.8.3 Photo-electric Absorption . . . . . . . . . . . . . 61

$2.8 .4 \quad$ Pair Production . . . . . . . . . . . . . . . . 62

2.9 Full EGS4 simulation . . . . . . . . . . . . . . . . . . . . . 62

$\begin{array}{ll}\text { 3. Silicon Detectors } & 67\end{array}$

3.1 Why use silicon as a PET detector? . . . . . . . . . . 67 
3.2 Basic Silicon Detector Operation _. . . . . . . . . . . 69

3.3 Silicon Detector Physics . . . . . . . . . . . . . . . . 72

3.3.1 Position Resolution . . . . . . . . . . . . . . . . 74

3.3.2 Timing Resolution . . . . . . . . . . . . . . . 75

3.3.3 Energy Resolution . . . . . . . . . . . . . . . . . 77

3.4 Our Silicon Detectors . . . . . . . . . . . . . . . . 80

3.4.1 VATAGP3 ASICS . . . . . . . . . . . . . . 83

3.4.2 Performance...................... 87

4. Experimental Setup 106

4.1 Experimental Setup Overview . . . . . . . . . . . . . . 106

4.2 Rotation Mechanism . . . . . . . . . . . . . . . 108

4.3 Choosing a Coincidence Timing Window . . . . . . . . . . . . . . 108

4.3.1 Coincidence Timing Set-up . . . . . . . . . . . . . . . . . . . . 112

4.4 Coincidence Timing Module Details . . . . . . . . . . . . . . . . . 115

4.5 Turntable Tilt . . . . . . . . . . . . . . . . . . 117

4.6 Aligning the silicon detectors . . . . . . . . . . . . . . 122

4.7 Post processing the data . . . . . . . . . . . . . . . . . . . 124

5. Image Reconstruction 130

5.1 Sinograms (Radon Transformation) . . . . . . . . . . . . . . . . 132

5.2 Iterative Image Reconstruction: The ML-EM Algorithm . . . . . . 133

5.3 3D ML-EM Positron Range Correction Algorithm . . . . . . . . . . . 142

5.4 Determining Detector Positions from Calibration Data . . . . . . . . 145

6. Experimental Results 
6.1 Introduction: Purpose of Chapter . . . . . . . . . . . . . . 155

6.2 Expected Image Resolution _. . . . . . . . . . . . . . . . 155

6.3 Comparison of Measured and Simulated Results . . . . . . . . . . . 158

6.4 Comparison to Other Results . . . . . . . . . . . . . . . 165

6.5 Artifacts from off-plane source measurements . . . . . . . . . . . . . 167

$\begin{array}{ll}\text { 7. Resolution Noise Study } & 173\end{array}$

7.1 Purpose of Chapter . . . . . . . . . . . . . . . . 173

7.2 Simulating Poisson Distributed Data . . . . . . . . . . . . 176

7.3 Testing the 3D ML-EM positron range correction algorithm: simple

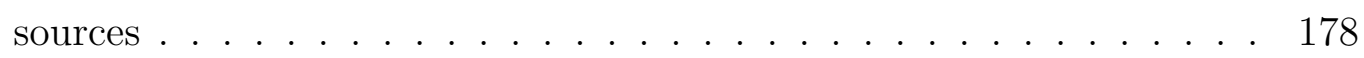

7.4 Testing the 3D ML-EM positron range correction algorithm: complex sources . . . . . . . . . . . . . . . . . . . . 184

7.5 Resolution versus Noise Study . . . . . . . . . . . . . . . . . 198

7.5.1 An algorithm for creating noise-resolution trade-off curves . . 204

7.5.2 Source geometry . . . . . . . . . . . . . . 207

7.5.3 Creating the PSFs ................ . . 210

7.5.4 Calculating the Noise-Resolution Curve . . . . . . . . . . . . . 227

7.6 Conclusions and Future Work . . . . . . . . . . . . . . . 233

$\begin{array}{ll}\text { BIBLIOGRAPHY } & 238\end{array}$ 


\section{LIST OF TABLES}

Table

Page

1.1 Physical properties of detector materials used in PET. *For LSO, BGO, and $\mathrm{NaI}(\mathrm{Tl})$ a 20 percent photo-multiplier tube (PMT) quantum efficiency is assumed $[4][5][6] . \ldots \ldots \ldots \ldots \ldots$

2.1 Maximum positron energies and half-lives of some commonly used positron emitters. . . . . . . . . . . . . . . . .

2.2 Relative physics interaction probabilities for 994,698 EGS4 interaction steps from 1000 simulated ${ }^{68} \mathrm{Ga}$ positron trajectories with initial kinetic energies randomly selected from the ${ }^{68} \mathrm{Ga}$ positron range distribution.

2.3 Fit parameters for $0 \mathrm{~T}$ positron range fits . . . . . . . . . . . . 48

2.4 Simulation results of the positron annihilation distribution projected onto the $\mathrm{x}$-axis for both a ${ }^{22} \mathrm{Na}$ and a ${ }^{68} \mathrm{Ga}$ point source for magnetic field strengths of $0 \mathrm{~T}$ and $7 \mathrm{~T}$ in water. The magnetic field is oriented in the positive $\mathrm{z}$ direction for the $7 \mathrm{~T}$ simulation results. The rms values are calculated using the magnitude of the positron range distribution.

$2.5 \mathrm{X}$-projection of the positron range for various positron emitters from four separate simulations. . . . . . . . . . . . . . . 
2.6 Simulation results of the positron annihilation distribution projected onto the $\mathrm{x}$-axis for both a ${ }^{22} \mathrm{Na}$ and $\mathrm{a}{ }^{68} \mathrm{Ga}$ point source for magnetic field strengths of $0 \mathrm{~T}$ and $7 \mathrm{~T}$ in lung tissue (water scaled by one third of the density). The magnetic field is oriented in the positive $\mathrm{z}$ direction for the $7 \mathrm{~T}$ simulation results. The rms values are calculated using the magnitude of the positron range distribution. . . . . . . .

3.1 $N_{\text {pairs }}=E_{\text {recoil }} / \eta$ where $\eta$ is the energy required to create an electronhole pair, I (nA) are rough estimates of the current assuming collection times $\tau$ of 200 ns (close to the $p^{+}$side) or 30 ns (close to the $n^{+}$ side), and $\Delta \mathrm{I}(\mathrm{pA})$ is the average variation of the current due to the uncertainty in the number of charge carriers $\sigma_{\text {pairs }} . \ldots . . . .$.

5.1 FWHM and FWTM values for projections onto the x-axis of a ${ }^{68} \mathrm{Ga}$ point source in water using the 3D ML-EM reconstructed images with and without the positron range correction (ML-EM iteration 200). Both distributions have been blurred by a $0.7 \mathrm{~mm}$ FWHM Gaussian system resolution. . . . . . . . . . . . . . . . . . . . . . . . 148

6.1 Measured and simulated results for a $0.25 \mathrm{~mm}$ diameter ${ }^{22} \mathrm{Na}$ point source and a $1.0 \mathrm{~mm}$ diameter ${ }^{68} \mathrm{Ga}$ point source in water. . . . . . . 167

6.2 Experimental results of the reconstructed positron range for both our small animal PET prototype and for Hammer and Christensen's measurement for ${ }^{22} \mathrm{Na}$ [55]. The diameter of the Hammer and Christensen source is $0.5 \mathrm{~mm}$ while a $0.25 \mathrm{~mm}$ diameter point source was used in this experiment. . . . . . . . . . . . . . . . 167 
6.3 Experimental results of the reconstructed positron range for both our small animal PET prototype and for Hammer and Christensen's mea-

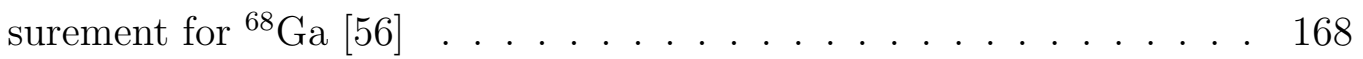

7.1 Table of the relative intensity of an individual rod in each region of the phantom shown in Fig. 7.10. The maximum activity is arbitrarily set

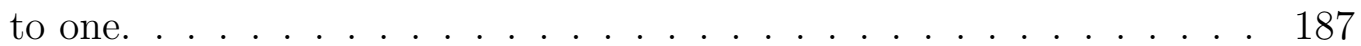

7.2 Table of PSF of FWHM and FWTM in the $\mathrm{x}$ and $\mathrm{z}$ directions. . . . . 220

\section{LIST OF FIGURES}

Figure

Page

1.1 An illustration of the basic physics principles of PET imaging. . . . . 2

1.2 The basic principle of PET detection. . . . . . . . . . . . . . . . 3

1.3 The three types of PET coincidence events: true, scattered, and random coincidence events. The dotted line in the figures represent the back-projected line-of-response (LOR) . . . . . . . . . . . .

1.4 Illustration of positioning errors that can occur when photons penetrate PET crystals at an angle relative to the face of the detector and penetration into neighboring detector elements prior to interacting. This depth of interaction results in back-projected LORs that do not pass through the positron-electron annihilation position. . . . . . 
1.5 Illustration of the error on the back-projected LORs due to photon acollinearity and the diameter of the PET detection ring. . . . . . . . 11

2.1 Block diagram of EGS4 simulation struction. . . . . . . . . . . . 22

2.2 Theoretical positron kinetic energy spectrum for various radioisotopes commonly used in PET. . . . . . . . . . . . . . . . . 26

2.3 Simulated energy spectrums for positron emitting radioisotopes commonly used in PET applications. The same number of events are simulated for each distribution. . . . . . . . . . . . . . . 27

2.4 Generic two body scattering diagram. . . . . . . . . . . . . . . 32

2.5 Energy loss per unit length $d E / d x$ for beta particles as a function of kinetic energy due to inelastic collisions and Bremsstrahlung radiation in water and lead. Image taken from [1]. . . . . . . . . . . . . . . 34

2.6 Example shape of $\frac{d E}{d x}$ versus penetration depth due to the interaction of a beta particle scattering through matter as it losses energy via inelastic collisions with atomic electrons. Image taken from [1]. . . .

2.7 Simulated positron trajectories projected onto a plane for ten $1900 \mathrm{keV}$ positrons emitted at various angles in water. . . . . . . . . .

2.8 Simulated positron trajectories projected onto a plane of ten $1900 \mathrm{keV}$ positrons emitted at various angles in water. A $7 \mathrm{~T}$ magnetic field is directed transverse to the plane. . . . . . . . . . . . . .

2.9 Histogram of random photon acollinearity angles. The distribution is Gaussian with a $0.5 \mathrm{~mm}$ FWHM. The bin width is 0.01 degrees. . . 
2.10 EGS4 simulation results of the x-projections of the ${ }^{68} \mathrm{Ga}$ positron annihilation distribution in water at $0 \mathrm{~T}$ and with a $7 \mathrm{~T}$ magnetic field applied in the z-direction. The area under the plots has been normalized to one. . . . . . . . . . . . . . . . . . . . 42

2.11 Two dimensional density histogram of the EGS4 simulation results of ${ }^{22} \mathrm{Na}$ positron annihilation positions in water at $0 \mathrm{~T} . \ldots . .$.

2.12 Projections of the ${ }^{22} \mathrm{Na} 0 \mathrm{~T}$ positron-electron annihilation distribution in water along the $\mathrm{x}, \mathrm{y}$, and $\mathrm{z}$ axes, respectively. . . . . . . . .

2.13 Two dimensional density histograms of the EGS4 simulation results of ${ }^{22} \mathrm{Na}$ positron annihilation positions in water at $7 \mathrm{~T}$ magnetic field applied transverse to the image plane (left) and a $7 \mathrm{~T}$ magnetic field in the positive z-direction (right) . . . . . . . . . . . . . 44

2.14 Projections of the ${ }^{22} \mathrm{Na} 7 \mathrm{~T}$ positron-electron annihilation distribution in water along the $\mathrm{x}, \mathrm{y}$, and $\mathrm{z}$ axes, respectively. . . . . . . . .

2.15 EGS4 simulation results of ${ }^{68} \mathrm{Ga}$ positron annihilation positions in wa-

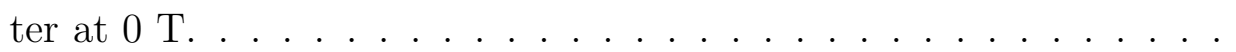

2.16 Projections of the ${ }^{68} \mathrm{Ga} 0 \mathrm{~T}$ positron-electron annihilation distribution in water along the $\mathrm{x}, \mathrm{y}$, and $\mathrm{z}$ axes, respectively. . . . . . . . . . . . . 47

2.17 EGS4 simulation results of ${ }^{68} \mathrm{Ga}$ positron annihilation positions in water at $7 \mathrm{~T}$ magnetic field (left) applied transverse to the image plane and (right) $7 \mathrm{~T}$ magnetic field in the positive $\mathrm{z}$-direction. . . . . . .

2.18 Projections of the ${ }^{68} \mathrm{Ga} 7 \mathrm{~T}$ positron-electron annihilation distribution in water along the $\mathrm{x}, \mathrm{y}$, and $\mathrm{z}$ axes, respectively. . . . . . . . . 
2.19 Fit of ${ }^{22} \mathrm{Na}$ EGS4 positron range annihilation x-projection of a point source at $0 \mathrm{~T}$ in water using the double exponential fit function 2.30 . The FWHM and FWTM are $0.06 \mathrm{~mm}$ and $0.60 \mathrm{~mm}$, respectively. Each bin in the plot corresponds to $0.005 \mathrm{~mm} . . . . . . . . . . . .$.

2.20 Fit of ${ }^{22} \mathrm{Na}$ EGS4 positron range annihilation x-projection of a point source at $7 \mathrm{~T}$ in water fit to a double Gaussian function. The FWHM and FWTM are $0.03 \mathrm{~mm}$ and $0.27 \mathrm{~mm}$, respectively. Each bin in the plot corresponds to $0.005 \mathrm{~mm}$. . . . . . . . . . . . . . .

2.21 Fit of ${ }^{68} \mathrm{Ga}$ EGS4 positron range annihilation x-projection of a point source at $0 \mathrm{~T}$ in water using the double exponential fit function 2.30 . The FWHM and FWTM are $1.02 \mathrm{~mm}$ and $5.45 \mathrm{~mm}$, respectively. Each bin in the plot corresponds to $0.005 \mathrm{~mm} \ldots \ldots . . . . . .$.

2.22 Fit of ${ }^{68} \mathrm{Ga}$ EGS4 positron range annihilation x-projection of a point source at $7 \mathrm{~T}$ in water using a standard double Gaussian fit function. The FWHM and FWTM are $0.84 \mathrm{~mm}$ and $2.28 \mathrm{~mm}$, respectively. Each bin in the plot corresponds to $0.005 \mathrm{~mm} . \ldots . . . . . . . .$.

2.23 Density plots of the positron-electron annihilation positions of a ${ }^{22} \mathrm{Na}$ point source in "lung" tissue (water scaled by $1 / 3$ of the density) at 0 $\mathrm{T}$ (left) and with a $7 \mathrm{~T}$ magnetic field applied transverse to the plane

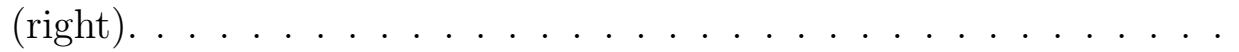

2.24 Density plots of the positron-electron annihilation positions of a ${ }^{68} \mathrm{Ga}$ point source in "lung" tissue (water scaled by $1 / 3$ of the density) at 0 $\mathrm{T}$ (left) and with a $7 \mathrm{~T}$ magnetic field applied transverse to the plane

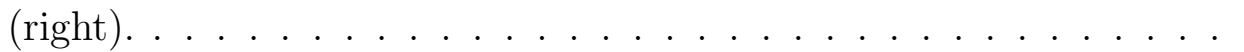


2.25 The absorption cross-section versus energy for the photo-electric effect, Compton scattering, and pair production for Si and Ge [33]. . . . . . 56

2.26 A diagram of the Compton scattering process. . . . . . . . . . 58

2.27 The energy of both the scattered photon and recoil electron energies versus the photon scattering angle for $511 \mathrm{keV}$ incident photons. . . . 58

2.28 Plot of Klein-Nishina scattering probability for silicon using $511 \mathrm{keV}$ incident photons. The area under the plot is normalized to one. . . . 60

2.29 Energy spectrum of the Compton recoil electrons using $511 \mathrm{keV}$ incident photons for one million events in silicon simulated in EGS4. . . . 60

2.30 Block diagram of total simulation. . . . . . . . . . . . . . 63

2.31 An illustration of the equivalence of rotating a symmetric positron source between two stationary silicon detectors (Left) and rotating the silicon detectors around a stationary symmetric positron source (Right). 65

2.32 An illustration that rotating the source between two stationary silicon detectors (Left) is not equivalent to rotating the detectors around a stationary, nonsymettric source (Right). . . . . . . . . . . . 
3.1 Diagram of the basics of silicon detector operations. Incident gamma radiation liberates a bound electron with sufficient energy that it ionizes additional atoms, creating a surge of electron-hole pairs. The number of free charge carriers is directly proportional to the initial kinetic energy of the recoil electron. The free charge carriers are swept out of the depletion region by an electric field created by a reverse bias voltage. The electrons and holes are collected at either end of the silicon detector volume and form the basis for determining the energy and position of the photon interaction. . . . . . . . . 70

3.2 Charge collection times for a fully depleted $1.0 \mathrm{~mm}$ thick silicon detector as a function of the interaction depth. $\mathrm{x}=0 \mathrm{~mm}$ corresponds to the $p^{+} \mathrm{n}$ boundary, and $\mathrm{x}=1.0 \mathrm{~mm}$ corresponds to the $n n^{+}$boundary. $\quad 77$

3.3512 pad $1.0 \mathrm{~mm}$ thick silicon detector. Each pad is $1.4 \mathrm{~mm} \times 1.4 \mathrm{~mm}$ in area. The detector is readout via four VATAGP3 ASICs that each readout 128 channels. . . . . . . . . . . . . . . . . . . .

3.4 Cross section of a single silicon pad. . . . . . . . . . . . .

3.5 Example block schematic of the electronic circuits used to trigger and shape the signal for two silicon channel. There are 128 identical channels on each VATAGP3 chip. Image taken from [44]. A resistor was added in parallel to the capacitor in the charge sensitive preamplifier. This resister will discharge the capacitor overtime with a characteristic time much greater than the total charge collection time. This resister was mistakenly omitted in the previous publication. . . . . . . . . . 
3.6 VATAGP3 readout cycle for an example silicon event. Image taken from $[44] . \ldots \ldots \ldots \ldots 6$

3.7 OSU1 (left) and OSU2 (right) pedestals versus channel number. Masked channels, OSU1: 1-10, 223,224 OSU2: 190,223,224 . . . . . . . . . 90

3.8 OSU1 and OSU2 pedestals at actual pad locations. Masked channels, OSU1: $1-10,223,224$ OSU2: 190, 223, $224 \ldots \ldots 90$

3.9 Histograms of OSU1 common mode noise per chip. . . . . . . . . . . 92

3.10 Histograms of OSU2 common mode noise per chip. As can be seen in the histograms there is a double peak that indicates the system is oscillating between two average noise values. The calculated average noise will fall between the two peaks. . . . . . . . . . . . . . . . . 92

3.11 Example process for determining the gain of each channel. (Left) Single pedestal and common mode noise corrected spectrum from example channel 281 from OSU1. (Right) Zoomed in view of the photoabsorption peak and associated Gaussian fit. The mean is extracted from the Gaussian fit and is used to determine the gain. . . . . . .

3.12 OSU1 and OSU2 gains versus channel number. Masked channels, OSU1: $1-10,223,224$ OSU2: 1-64, 190, 223, $224 \ldots \ldots 94$

3.13 OSU1 and OSU2 gains versus channel number. Masked channels, OSU1: 1-10, 223, 224 OSU2: 1-64, 190, 223, $224 \ldots \ldots \ldots$

3.14 OSU1 pedestal and common mode noise corrected ${ }^{241} \mathrm{Am}$ spectrums for each channel (Left) without a gain correction and (Right) with a gain correction. Masked channels, OSU1: 1-10, 223, 224 OSU2: 1-64, $190,223,224 \ldots \ldots \ldots \ldots \ldots \ldots$ 
3.15 OSU2 pedestal and common mode corrected ${ }^{241} A m$ spectrums for each channel (Left) without a gain correction and (Right) with a gain correction. Masked channels, OSU1: 1-10, 223, 224 OSU2: 1-64, 190, 223,

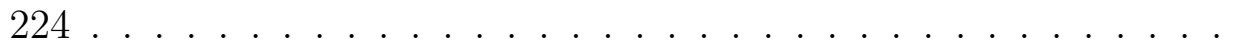

3.16 Sigma value of Gaussian resolution peak versus channel for OSU1 and OSU2, respectively. . . . . . . . . . . . . . .

3.17 Sigma value of Gaussian resolution peak at the actual channel location for OSU1 and OSU2, respectively. . . . . . . . . . . .

3.18 OSU1 and OSU2 pedestal, common mode noise, and gain corrected ${ }^{241} \mathrm{Am}$ total energy spectrums. . . . . . . . . . . . . . . . .

3.19 Photograph of the wirebond testing apparatus used to determine the reason why the wire bonds were breaking in the $8 \mathrm{~T}$ magnetic field, and to test if encapsulating the wirebonds could prevent the wirebonds from breaking. The holes drilled into the top of the device on the right are used to view the wirebonds. . . . . . . . . . . . . . . . . . 100

3.20 Schematic of a single wirebond circuit used to test the wirebonds in the 8 T magnet field. . . . . . . . . . . . . . . . . . 100

3.21 Image of the two magnetic field orientations tested on the wirebonds with alternating current $I(t)$ and the associated force on the wirebond $F(t)$. The magnetic field $B$ has a constant magnitude of 8 T. . . . . . 101

3.22 Image of wire bonds broken at the feet due to mechanical stress. . . . 103

$3.23{ }^{241} \mathrm{Am}$ energy spectrum at $0 \mathrm{~T}$ and $8 \mathrm{~T}$. The two distributions overlap almost perfectly. . . . . . . . . . . . . . . . 105

4.1 Cross-sectional diagram of the small animal PET test-bench. . . . . . 107 
4.2 Photograph of the small animal PET test-bench as viewed from above relative to the cross-sectional diagram shown in Fig. 4.1. . . . . . . . 107

4.3 Block diagram of source rotation mechanism used to collect data with full angular sampling. The fiber optic mount is use to confirm that the source is properly rotated. . . . . . . . . . . . . . . . . 109

4.4 Coincidence measurement setup consisting of a collimated ${ }^{18} \mathrm{~F}$ line source position in the center of the FOV of the two silicon detectors. The output triggers from OSU1 were sent directly to the START input of a time to digital converter (TDC), while the output triggers from OSU2 were delayed by 384 ns before being sent to the STOP input of the TDC. The TDC output (the time difference between the two trigger signals) is digitized by a multi-channel analyzer (MCA). . . . 110

4.5 Coincidence measurement performed at the University of Michigan using a collimated ${ }^{18} \mathrm{~F}$ line source at the center of the field of view. Note: The 200 ns coincidence timing window was chosen for the previous small animal PET prototype. A 300 ns timing window was chosen for the setup described in the document. Image taken from [44]. . . . . . 111

4.6 Block diagram of the small animal PET prototype readout electronics. 113

4.7 Small animal PET coincidence timing set-up . . . . . . . . . . . . . . 115

4.8 Turntable tilt geometry for a tilt where the normal vector to the plane always points in the same direction. . . . . . . . . . . . . . 118

4.9 Turntable tilt geometry for a tilt where the normal vector to the plane precesses about the ideal rotation axis. This is the turntable geometry of our setup. . . . . . . . . . . . . . . . . . . 119 
4.10 Measurement results of the deviation distance $X$ of the turntable. . . 120

4.11 Geometry used to calculate the tilt angle. . . . . . . . . . . . . . . . 120

4.12 Plot of $h(x)$, the offset from the detector plane, for source positions between -100 and 100 mm radius. . . . . . . . . . . . . . . . . . 122

4.13 Coincidence frequency versus turntable angle used to confirm correct detector alignment. . . . . . . . . . . . . . . .

4.14 Comparison of the hit map between simulated and real data for the source at angle 60 degrees. The excellent agreement between the simulated and real data is further evidence of a properly aligned system.

4.15 Diagram of source rotation transformation. The primed variables have been rotated by the angle $\theta$. . . . . . . . . . . . . . . . 126

4.16 2D density plot of the transformed $x^{\prime}$ and $y^{\prime}$ photon annihilation posi-

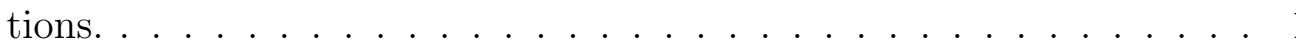

4.17 Histogram of the penetration depth of each photon interaction calculated using $\sqrt{x^{2}+y^{2}} \ldots \ldots \ldots \ldots \ldots$

4.18 EGS4 simulated silicon penetration depth fit to an exponential. The decay parameter of the exponential is the attenuation coefficient which is $0.202 \mathrm{~cm}^{-1}$ extracted from the fit which is in excellent agreement with the the accepted value of $0.20 \mathrm{~cm}^{-1} \ldots \ldots \ldots$. . . . . . . . 129

5.1 Example of EGS4 simulated data of three ${ }^{22} \mathrm{Na}$ point sources at $0 \mathrm{~T}$ in water reconstructed with the analytical filtered back projection method (left) [48] and 20 iterations of the ML-EM algorithm. . . . . . . . . . 131 
5.2 Figure illustrating the variables of a sinogram. The distance $r$ is the shortest distance from the origin to the LOR, and $\theta$ is the angle with respect to a user defined axis. . . . . . . . . . . . . . . . . . 132

5.3 Example sinograms of a 0.25 diameter ${ }^{22} \mathrm{Na}$ (left) and 1.0 diameter ${ }^{68} \mathrm{Ga}$ point source point source located $6 \mathrm{~mm}$ off the center of the FOV embedded in water at $0 \mathrm{~T}$. One can note that the sources will be reconstructed by 180 degrees apart due to the difference in phase of the sinogram shapes. . . . . . . . . . . . . . . .

5.4 Example sinograms of two 0.25 diameter ${ }^{22} \mathrm{Na}$ point source point source located $6 \mathrm{~mm}$ off the center of the FOV embedded in water at $0 \mathrm{~T}$ on opposite sides of the center of the field of view. . . . . . . . . . .

5.5 The first step of the ML-EM algorithm takes the current image estimate $\lambda^{(k)}$ and forward projects the image into the sinogram space creating $\tilde{y}^{(k)} \ldots \ldots \ldots \ldots \ldots \ldots \ldots \ldots$

5.6 In second step of the ML-EM algorithm the measured data $y(j)$ (in sinogram form) is compared to the current estimate of the image sinogram $\tilde{y}^{(k)}$ by a simple, pixel-by-pixel division resulting in a estimated sinogram. . . . . . . . . . . . . . . . .

5.7 In step 3 of the ML-EM algorithm, the estimated sinogram from step 2 is forward projected into the image space by the use of the system matrix $a(i, j)$ to create a temporary image $C^{(k)}$. . . . . . . . . . 140 
5.8 The $C^{(k)}$ image is used to modify the current estimate $\lambda^{(k)}$ by multiplying each pixel element of the current image estimate $\lambda^{(k)}$ by the temporary matrix $C^{(k)}$ resulting in the next ML-EM image estimate $\lambda^{(k+1)} \ldots \ldots \ldots \ldots \ldots \ldots \ldots$

5.9 The next step of the ML-EM algorithm is the same as step 1, but with a new guess for the image estimate $\lambda^{(k+1)}$. The ML-EM algorithm is repeated until the image has sufficiently converged. . . . . . . . . . . 141

5.10 FWHM and FWTM resolution verses ML-EM iteration number for a $0.25 \mathrm{~mm}{ }^{22} \mathrm{Na}$ point source embedded in water. . . . . . . . . . 142

5.11 Central 3D slice of ${ }^{68} \mathrm{Ga}$ point sources in water at $0 \mathrm{~T}$ with (left) and without (right) the positron range correction for ML-EM iteration 200. 100,000 Poisson distributed events were used and the final reconstructed images where blurred with a 3D $0.7 \mathrm{~mm}$ FWHM Gaussian

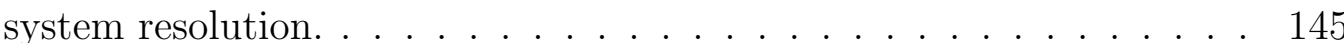

5.12 Projections along the $\mathrm{x}$-axis of the images shown in Fig. 5.11. The plots are normalized such that the value of the peak in one. The FWHM values are $0.77 \mathrm{~mm}$ and $1.92 \mathrm{~mm}$ for the corrected and the uncorrected data, respectively. The FWTM values are $1.55 \mathrm{~mm}$ and $6.12 \mathrm{~mm}$ for the corrected and uncorrected data, respectively. . . . . 146 
5.13 Central 3D slice of ${ }^{68} \mathrm{Ga}$ point sources in water at $7 \mathrm{~T}$ with (left) and without (right) the positron range correction for ML-EM iteration 200 using three orthogonal $7 \mathrm{~T}$ data sets simultaneously to reconstruct the images. 100,000 Poisson events were used and the final reconstructed images where blurred with a 3D $0.7 \mathrm{~mm}$ FWHM Gaussian system resolution. ...................... 146

5.14 Slices along the $\mathrm{x}$-axis through the maxima of the images in Fig. 5.13. The plots are normalized such that the peak values are one. The FWHM values are $0.75 \mathrm{~mm}$ and $1.42 \mathrm{~mm}$ for the corrected and the uncorrected data, respectively. The FWTM values are $1.52 \mathrm{~mm}$ and $3.50 \mathrm{~mm}$ for the corrected and uncorrected data, respectively. . . . . 147

5.15 FWHM and FWTM resolutions of a ${ }^{68} \mathrm{Ga}$ point source in water at $0 \mathrm{~T}$ versus iteration number using the ML-EM 3D positron range correction algorithm. The image has been blurred for a $0.7 \mathrm{~mm}$ Gaussian system resolution. . . . . . . . . . . . . . . . . . 147

5.16 FWHM and FWTM resolutions of a ${ }^{68} \mathrm{Ga}$ point source in water at $7 \mathrm{~T}$ versus iteration number using the ML-EM 3D positron range correction algorithm reconstructed with three orthogonal data sets with the positron range distribution from $\mathrm{x}, \mathrm{y}$, and $\mathrm{z}$ magnetic field orientations. The image has been blurred for a $0.7 \mathrm{~mm}$ Gaussian system resolution. 148

5.17 The data that was used to create this sinogram is used in the detector estimation algorithm. The sinogram shown here is not corrected for detector shifts. . . . . . . . . . . . . . . . . . 151 
5.18 This sinogram is created using the same data with the detector shifts determined by the detector estimation algorithm. The shifts in $\mathrm{x}$ and $\mathrm{y}$ are $(-1.71 \mathrm{~mm}, 0.29 \mathrm{~mm})$ for the first detector and $(-2.23 \mathrm{~mm}, 0.29$ $\mathrm{mm}$ ) for the second detector. The discontinuities in the sinogram are no longer present. . . . . . . . . . . . . . . . . . 152

5.19 This sinogram is created without any detector shift corrections assuming the detectors are positioned perfectly. On close inspection it can be observed that this figure has rough discontinuities along its length.

5.20 This sinogram is created using the same data that was used to create the sinogram in Fig. 5.19, but uses the detector shifts of $(-1.71 \mathrm{~mm}$, $0.29 \mathrm{~mm})$ and $(-2.23 \mathrm{~mm}, 0.29 \mathrm{~mm})$. Note that the discontinuities are no longer present. The difference in the final reconstructed images is $1.0 \mathrm{~mm}$ FWHM using the uncorrected sinogram compared to $0.8 \mathrm{~mm}$ FWHM using the corrected sinogram. . . . . . . . . . . . .

6.1 Left: two dimensional density histogram of the positron-electron annihilation positions in the detector plane of a ${ }^{18} \mathrm{~F} 0.254 \mathrm{~mm}$ diameter liquid source contained within a $0.127 \mathrm{~mm}$ thick steel syringe. Right: The projection of Fig. 6.1 onto the x-axis. . . . . . . . . . . . . . . 156

6.2 ML-EM reconstruct image (iteration 20) of the EGS4 simulated ${ }^{18} \mathrm{~F}$ source contained in a $0.254 \mathrm{~mm}$ inner diameter steel syringe. . . . . . 157

6.3 The x-projection of the image shown in Fig. $6.2 \ldots$. . . . . . . . 158

6.4 Positron emitting sources embedded in a water equivalent plastic of density $1.08 \mathrm{~g} / \mathrm{cm}^{3}$ manufactured by Eckert \& Ziegler Isotope Products [53]. . . . . . . . . . . . . . . . . . 159 
6.5 ML-EM reconstructed image of measured data (left image) and simulated data (middle image) of a $0.25 \mathrm{~mm}$ diameter ${ }^{22} \mathrm{Na}$ point source encased in a water equivalent plastic at $0 \mathrm{~T}$. The image on the right is the comparison of the measured and simulated data projected onto the x-axis. . . . . . . . . . . . . . . . 160

6.6 ML-EM reconstructed image of measured data (left image) and simulated data (middle image) of a $0.25 \mathrm{~mm}$ diameter ${ }^{22} \mathrm{Na}$ point source encased in a water equivalent plastic with a $7 \mathrm{~T}$ magnetic field applied transverse to the plane of the image. The image on the right is the comparison of the measured and simulated data projected onto the

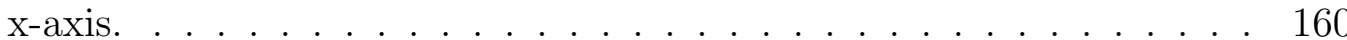

6.7 ML-EM reconstructed image of measured data (left image) and simulated data (middle image) of two $0.25 \mathrm{~mm}$ diameter ${ }^{22} \mathrm{Na}$ point source separated by $1.5 \mathrm{~mm}$ from their centers. The sources are encased in a water equivalent plastic at $0 \mathrm{~T}$. The image on the right is the comparison of the measured and simulated data projected onto the x-axis.

6.8 ML-EM reconstructed image of measured data (left image) and simulated data (middle image) of two $0.25 \mathrm{~mm}$ diameter ${ }^{22} \mathrm{Na}$ point source separated by $1.5 \mathrm{~mm}$ from their centers. The sources are encased in a water equivalent plastic at $7 \mathrm{~T}$. The image on the right is the comparison of the measured and simulated data projected onto the x-axis. . 162

6.9 Comparisons of the measured (left) and simulated (right) x-projections of the dual ${ }^{22} \mathrm{Na}$ study from figures 6.7 and $6.8 \ldots \ldots . \ldots 3$ 
6.10 ML-EM reconstructed image of measured data (left image) and simulated data (middle image) of a $1.0 \mathrm{~mm}$ diameter ${ }^{68} \mathrm{Ga}$ point source encased in a water equivalent plastic at $0 \mathrm{~T}$. The image on the right is the comparison of the measured and simulated data projected onto the x-axis. . . . . . . . . . . . . . . . 163

6.11 ML-EM reconstructed image of measured data (left image) and simulated data (middle image) data of $1.0 \mathrm{~mm}$ diameter ${ }^{68} \mathrm{Ga}$ point source encased in a water equivalent plastic with a $7 \mathrm{~T}$ magnetic field applied transverse to the image plane. The image on the right is the comparison of the measured and simulated data projected onto the x-axis. . . 164

6.12 ML-EM image of two $1.0 \mathrm{~mm}$ diameter ${ }^{68} \mathrm{Ga}$ point sources separated by $3.6 \mathrm{~mm}$ from their centers at $0 \mathrm{~T}$ (left) and in a $7 \mathrm{~T}$ (right) magnetic field directed transverse to the image plane. The source on the right possess a factor of 10 higher activity than the source on the left. Both of the image are reconstructed from measured data. . . . . . . . . . 165

6.13 The x-projections of two $1.0 \mathrm{~mm}$ diameter ${ }^{68} \mathrm{Ga}$ point sources separated by a $3.6 \mathrm{~mm}$ from their centers at 0 Tesla (left) and in a $7 \mathrm{~T}$ magnetic field directed transverse to the image plane (right). The source on the left possesses 10 times the activity as the source on the left. . . . . . 166 
6.14 Left: Measured data of four $1.0 \mathrm{~mm}$ diameter ${ }^{68} \mathrm{Ga}$ point sources positioned within the detector plane and at $1 \mathrm{~mm}, 2 \mathrm{~mm}$, and $3 \mathrm{~mm}$ outside of the detection plane at $0 \mathrm{~T}$. Right: Measured data of four 1.0 $\mathrm{mm}$ diameter ${ }^{68} \mathrm{Ga}$ point sources positioned within the detector plane and at $1 \mathrm{~mm}, 2 \mathrm{~mm}$, and $3 \mathrm{~mm}$ outside of the detection plane at $7 \mathrm{~T}$ (applied transverse to the image plane). The artifacts from off-plane sources are more sharply defined in the strong magnetic field. . . . .

6.15 Left: Projection along the x-axis of the measured data of four $1.0 \mathrm{~mm}$ diameter Ga-68 point sources positioned within the detector plane and at $1 \mathrm{~mm}, 2 \mathrm{~mm}$, and $3 \mathrm{~mm}$ outside of the detection plane at $0 \mathrm{~T}$. Right: Projection along the x-axis of the measured data of four 1.0 mm diameter Ga-68 point sources positioned within the detector plane and at $1 \mathrm{~mm}, 2 \mathrm{~mm}$, and $3 \mathrm{~mm}$ outside of the detection plane at $7 \mathrm{~T}$ (applied transverse to the image plane). The artifacts from off-plane sources are more sharply defined in the strong magnetic field. . . . . . 170

6.16 A diagram demonstrating the LORs that can be detected using a). a stack of 2D PET detectors to create 3D images and b). the LORs that would be detected in a fully 3D PET system. . . . . . . . . . . . . . . 171 
7.1 This figure illustrates the three cases studied in this chapter. The general shape of the positron range distributions are illustrated in each case. Each case uses the ${ }^{68} \mathrm{Ga}$ positron range distribution with or without the application of a $7 \mathrm{~T}$ magnetic field. Case 1: The $0 \mathrm{~T}^{68} \mathrm{Ga}$ positron range distribution in water is used to simulate the data and as input to the reconstruction algorithm. Case 2: The ${ }^{68} \mathrm{Ga}$ positron range distribution in water in the presence of a $7 \mathrm{~T}$ magnetic field directed transverse to the image planes (axially) is used to simulate the data and as input to the reconstruction algorithm. Case3: Three independent data sets are simulated with the ${ }^{68} \mathrm{Ga}$ positron range distribution in water at $7 \mathrm{~T}$ along the $\mathrm{x}, \mathrm{y}$, and $\mathrm{z}$ axis. The three data sets are reconstructed simultaneously using the $\mathrm{x}, \mathrm{y}$, and $\mathrm{z}$ positron range distributions of ${ }^{68} \mathrm{Ga}$ in water. . . . . . . . . . . . . . . 175

7.2 Example of sinograms created from a single pixel point source located $6.0 \mathrm{~mm}$ from the center of the FOV blurred with the $0 \mathrm{~T}^{68} \mathrm{Ga}$ positron range without (left) and with (right) Poisson distributed events. The sinogram with Poisson distributed events contains 100,000 LORs. The source image was also blurred with a $0.7 \mathrm{~mm}$ Gaussian system resolution and the $0.41 \mathrm{~mm}$ blur due to photon acollinearity prior to being forward projected into sinogram space in order to compare to the sinograms created from the EGS4 simulation. . . . . . . . . . . . . . . 177

xxix 
7.3 Sinogram created from the full EGS4 simulation of a ${ }^{68} \mathrm{Ga}$ point source in water at $0 \mathrm{~T}$ located $6.0 \mathrm{~mm}$ from the center of the FOV. This sinogram is used to compare to the sinograms created using the convolution technique discussed in this section. . . . . . . . . . . . . 177

7.4 Projection of the sinogram shown on the right in Fig. 7.2 and the sinogram from the full EGS4 simulation shown in Fig. 7.3 onto the angular axis. The differences in the sinograms is due to the discrete angular intervals at which the EGS4 data was collected in comparison to the blurring-convolution method that assumes a complete detector

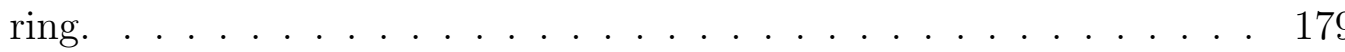

7.5 Left: 3D ML-EM reconstruction with the positron range correction turned off for simulated data at $0 \mathrm{~T}$ for four $1.0 \mathrm{~mm}{ }^{68} \mathrm{Ga}$ points positioned within the detection plane and at $0.4 \mathrm{~mm}, 0.8 \mathrm{~mm}$, and 1.2 mm outside of the detection plane. Right: 3D ML-EM reconstruction with the positron range correction turned off for simulated data at $7 \mathrm{~T}$ for four $1.0 \mathrm{~mm}{ }^{68} \mathrm{Ga}$ points positioned within the detection plane and at $0.4 \mathrm{~mm}, 0.8 \mathrm{~mm}$, and $1.2 \mathrm{~mm}$ outside of the detection plane. Each image contains 100,000 Poisson distributed events. These images show that the artifacts from off-plane sources are more sharply defined in a strong magnetic field directed transverse to the image plane. . . . . . 
7.6 Left: 3D ML-EM reconstruction with the positron range correction turned on for data simulated at $0 \mathrm{~T}$ for four $1.0 \mathrm{~mm}{ }^{68} \mathrm{Ga}$ point sources positioned within the detection plane and at $0.4 \mathrm{~mm}, 0.8 \mathrm{~mm}$, and $1.2 \mathrm{~mm}$ outside of the detection plane. Right: 3D ML-EM reconstruction with the positron range correction turned on (using the $7 \mathrm{~T}$ (axial) correction) for simulated data at $7 \mathrm{~T}$ for four $1.0 \mathrm{~mm}{ }^{68} \mathrm{Ga}$ point sources positioned within the detection plane and at $0.4 \mathrm{~mm}, 0.8 \mathrm{~mm}$, and $1.2 \mathrm{~mm}$ outside of the detection plane. Each image contains 100,000 Poisson distributed events. The positron range contribution to the final image resolution has been completely removed for both the $0 \mathrm{~T}$ and 7 T data. . . . . . . . . . . . . . . . . 181

7.7 Left: The projection of the two images contained in Fig. 7.5 onto the $\mathrm{x}$-axis demonstrating that the artifacts from positron annihilations within the current image plane from sources located in adjacent planes are more highly defined in strong magnetic fields. Right: The projection of the two images contained in Fig. 7.6 onto the x-axis demonstrating that the positron range has been completely removed. The artifacts still present from off-plane sources is due to the $0.7 \mathrm{~mm}$ FWHM Gaussian system resolution and the $0.41 \mathrm{~mm}$ photon acollinearity uncertainty. 182

7.8 Left: 3D ML-EM positron range corrected image using three, independent magnetic field orientations along the $\mathrm{x}, \mathrm{y}$, and $\mathrm{z}$ axes. This image has been blurred with the $0.7 \mathrm{~mm}$ FWHM Gaussian system resolution and the $0.41 \mathrm{~mm}$ uncertainty introduced by the photon acollinearity. Right: The $\mathrm{x}$ projection of the image on the left. . . . . . . . . . . . . 183 
7.9 A photograph of a complex liquid source holder that is used to characterize the performance of PET scanners (also known as a Jaszczak phantom [58]). The source distribution simulated in this section is based off of this phantom, but with two neighboring planes rotated at an angle with respect to each other. Photograph courtesy of Sang-June Park $[44] . \ldots \ldots \ldots \ldots \ldots \ldots \ldots \ldots \ldots \ldots \ldots \ldots$

7.10 Phantom source distribution for plane 5 and 7 of a ten plane phantom. Each plane is $0.4 \mathrm{~mm}$ thick. All other planes do not contain positron

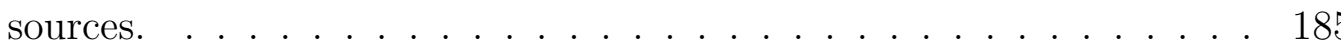

7.11 A simple illustration of the phantom source consisting of ten $0.4 \mathrm{~mm}$ thick planes. Only planes 5 and 7 contain sources. Note that the sources in planes 5 and 7 are rotated with respect to each other such that positron penetration from neighboring planes will be evident. .

7.12 Normalized log-likelihood (multiplied by -1 and normalized to converge to 1.0) of the reconstructed phantom versus iteration number for the three cases using the 3D ML-EM positron range correction algorithm.

7.13 Images of phantom planes 5, 6, and 7 (from left to right) blurred with the $0 \mathrm{~T}^{68} \mathrm{Ga}$ positron range distribution in water. Note that plane six does not have any sources and that all annihilation positions are from the two neighboring planes. These are not reconstructed images, but instead are images of the phantoms blurred by the positron range. . 
7.14 Images of phantom planes 5, 6, and 7 (from left to right) blurred with the $7 \mathrm{~T}^{68} \mathrm{Ga}$ positron range distribution in water with a $7 \mathrm{~T}$ magnetic field oriented in the z-direction. Note that plane six does not have any sources and that all annihilation positions are from the two neighboring planes. These are not reconstructed images, but instead are images of the phantoms blurred by the positron range. . . . . . . . . . . . . . . 190

7.15 Images of phantom planes 5, 6, and 7 blurred with the ${ }^{68}$ Ga positron range distribution in water with a $7 \mathrm{~T}$ magnetic field oriented in the y-direction. Note that plane six does not have any sources and that all annihilation positions are from the two neighboring planes. These are not reconstructed images, but instead are images of the phantoms blurred by the positron range. . . . . . . . . . . . . . . . . . . . . 191

7.16 Left: Example sinogram created by forward projecting plane 5 of the $0 \mathrm{~T}^{68} \mathrm{Ga}$ blurred phantom. Right: The same sinogram after varying each pixel by a random number from the Poisson distribution. . . . . 191

7.17 Phantom planes 5, 6, and 7 using the $0 \mathrm{~T}^{68} \mathrm{Ga}$ positron range in water for the 3D ML-EM positron range correction algorithm, respectively . 192

7.18 Phantom planes 5, 6, and 7 using the $7 \mathrm{~T}^{68} \mathrm{Ga}$ positron range with the magnetic field oriented in the axial z-direction using the 3D ML-EM positron range correction algorithm. . . . . . . . . . . .

7.19 Phantom planes 5, 6, and 7 using three orthogonal $7 \mathrm{~T}$ magnetic field orientations of the ${ }^{68} \mathrm{Ga}$ positron range in water for the $3 \mathrm{D}$ ML-EM positron range correction algorithm, respectively. . . . . . . . . 
$7.200 \mathrm{~T}$ (left), $7 \mathrm{~T}$ (3 orientations) (middle), and $7 \mathrm{~T}$ (axial) images zoomed in on the small $1.2 \mathrm{~mm}$ diameter sources from the reconstructed images shown in Figs. 7.17 through 7.19. . . . . . . . . . . . . . . . 194

7.21 Images of $1.2 \mathrm{~mm}$ rods that have been corrected for the positron range with the 3D ML-EM algorithm positron range correction and post blurred for a $0.7 \mathrm{~mm}$ Gaussian system resolution: (left) $0 \mathrm{~T}$, (middle) $7 \mathrm{~T}$ with three orientations, and (left) a $7 \mathrm{~T}$ magnetic field in the axial z-direction.

7.22 The red line represents the projections shown in the following figures for the 1.2 mm rods shown in Figs. 7.24 through 7.26. . . . . . . .

7.23 Images of $1.2 \mathrm{~mm}$ rods that have been corrected for the positron range with the 3D ML-EM algorithm positron range correction and post blurred for a $0.7 \mathrm{~mm}$ Gaussian system resolution: (left) $0 \mathrm{~T}$, (middle) $7 \mathrm{~T}$ with three orientations, and (left) a $7 \mathrm{~T}$ magnetic field in the axial z-direction.

7.24 Slices through the $1.2 \mathrm{~mm}$ rods of the positron range corrected images at $0 \mathrm{~T}$ without blurring for the $0.7 \mathrm{~mm}$ FWHM system resolution (left) and with blurring for the system resolution (right). . . . . . . . 196

7.25 Slices through the $1.2 \mathrm{~mm}$ rods of the positron range corrected images at $7 \mathrm{~T}$ (three magnetic field orientations) without blurring for the $0.7 \mathrm{~mm}$ FWHM system resolution (left) and with blurring for the system resolution (right). . . . . . . . . . . . . . . . . . . 196 
7.26 Slices through the $1.2 \mathrm{~mm}$ rods of the positron range corrected images at $7 \mathrm{~T}$ (axial z-direction) without blurring for $0.7 \mathrm{~mm}$ FWHM the system resolution (left) and with blurring for the system resolution

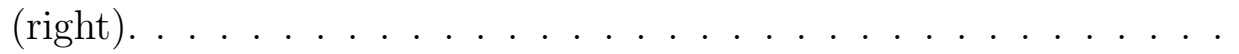

7.27 The 3D ML-EM positron range corrected image of a ${ }^{68} \mathrm{Ga}$ point source possessing $1 / 10$ of the activity of the ${ }^{68} \mathrm{Ga}$ constant background distribution at $0 \mathrm{~T}$ (in water) in the absence of Poisson distributed events (left) at 1000 iterations and (right) at 6000 iterations. The source is located in voxel $(40,40)$, and is surrounded by the the black ring in the images. . . . . . . . . . . . . . . . . . . . . 200

7.28 The 3D ML-EM positron range corrected image of a ${ }^{68} \mathrm{Ga}$ point source possessing $1 / 10(1 / 100$ is used later in the study - $1 / 10$ is used here such that the source is more easily identified) of the activity of the ${ }^{68} \mathrm{Ga}$ constant background distribution at $0 \mathrm{~T}$ (in water) with Poisson distributed events (left) at 1000 iterations and (right) at 6000 iterations. The source is located in voxel $(40,40)$, and is surrounded by the red ring in the images. For both cases the source is obscured by the variation in the background events. This plot also demonstrates that the variation in the noise increases as the resolution increases. . . . . 201 
7.29 A projection along the bin passing through the ${ }^{68} \mathrm{Ga}$ point source in the constant ${ }^{68} \mathrm{Ga}$ background distribution from the images in Fig. 7.27 without (blue) and with (red) Poisson distributed events. The projection on the left is for 1000 iterations and the image on the right is for 6000 iterations of the 3D ML-EM positron range correction algorithm. For both cases the source is obscured by the variation in the background events. This plot also demonstrates that the variation in the noise increases as the resolution increases. . . . . . . . . . . . 202

7.30 Example noise versus resolution plot that displays the characteristic trade-off between the resolution and the noise for two hypothetical systems. The system with the red resolution noise curve will yield images with lower noise for any given system resolution. . . . . . . . 203

7.31 Left: Central slice of the uniform background source. Right: Central slice of the uniform background source blurred for the $0 \mathrm{~T}^{68} \mathrm{Ga}$ positron range in water. . . . . . . . . . . . . . . . . . . . 208

7.32 Background blurred with two orientations of the $7 \mathrm{~T}$ magnetic field. The magnetic field is oriented in the positive y-direction for the image on the left, and in the positive x-direction for the image on the right. Note the blurring of the background distribution in the directions transverse to the magnetic field vector. . . . . . . . . . . . 208

7.33 Background blurred with the ${ }^{68} \mathrm{Ga}$ positron range distribution in water with a $7 \mathrm{~T}$ magnetic field in the axial z-direction. Note that the blurring along the edges of the source have been significantly reduced. . . . . . 209 
7.34 Sinogram of the $0 \mathrm{~T}^{68} \mathrm{Ga}$ positron range blurred background without (left) and with (right) Poisson distributed events applied. . . . . . . . 209

7.35 Reconstructed images with $10^{8}$ Poisson distributed events for $0 \mathrm{~T}$ and 7 $\mathrm{T}$ (3 orientations) simulated data for 1000 iterations of the 3D ML-EM positron range correction algorithm. . . . . . . . . . . . . 210

7.36 A graphical representation of the projection slices shown in this section.211

$7.370 \mathrm{~T}$ and $7 \mathrm{~T}$ (3 orientations) PSFs in the xy-plane. Note that the positron range correction is having difficulty in this environment. In particular note the crossing artifact in the $7 \mathrm{~T}$ data suggesting that the algorithm is slow to remove the positron range. . . . . . . . . . 213

$7.380 \mathrm{~T}$ and $7 \mathrm{~T}$ (3 orientations) PSFs in the xz-plane. Note that the positron range correction is having difficulty in this environment. In particular note the crossing artifact in the $7 \mathrm{~T}$ data suggesting that the algorithm is slow to remove the positron range. . . . . . . . . . 214

7.39 Slice through maximum in $\mathrm{x}$ and $\mathrm{z}$ direction for $0 \mathrm{~T}$ and $7 \mathrm{~T}$ (3 orientation) data. The widths for the $\mathrm{x}$-slices are: $\mathrm{FWHM}_{0 T}=1.50 \mathrm{~mm}$ $\mathrm{FWTM}_{0 T}=3.19 \mathrm{~mm}, \mathrm{FWHM}_{7 T}=1.40 \mathrm{~mm}, \mathrm{FWTM}_{7 T}=2.84 \mathrm{~mm}$. The width for the z-slices are: $\mathrm{FWHM}_{0 T}=0.87 \mathrm{~mm}, \mathrm{FWTM}_{0 T}=2.13$ $\mathrm{mm}, \mathrm{FWHM}_{7 T}=0.96 \mathrm{~mm}, \mathrm{FWTM}_{7 T}=1.58 \mathrm{~mm} \ldots \ldots \ldots . . .214$

7.40 FWHM resolution along the $\mathrm{x}$-axis versus iteration for a small perturbative source in a constant background at both $0 \mathrm{~T}$ and $7 \mathrm{~T}$ using three orthogonal magnetic field orientations. . . . . . . . . . . . 215

7.41 Resolution versus iteration number for $0 \mathrm{~T}$ ML-EM 3D positron range correction algorithm. . . . . . . . . . . . . . . 216 
7.42 The $\mathrm{x}$ and $\mathrm{z}$ projections through the maxima of the $0 \mathrm{~T}$ and $7 \mathrm{~T}(3$ orientation) PSFs reconstructed with 3400, 3600, and 3800 iteration. The purpose of this plot is to show that all three iterations numbers are close matches to the $0 \mathrm{~T}$ PSF. Fig. 7.44 shows why iteration 3600 iterations (black line) of the 3D ML-EM positron range correction algorithm yields the closest match of the $7 \mathrm{~T}$ (3 orientations) PSF to the 0 T PSF. . . . . . . . . . . . . . . . 218

7.43 The $\mathrm{x}$ and $\mathrm{z}$ projections through the maxima of the $0 \mathrm{~T}$ and $7 \mathrm{~T}$ (axial) PSFs reconstructed with 3200, 3400, and 3600 iterations. The purpose of this plot is to show that all three iterations numbers are the closest match to the 0 T PSF. Fig. 7.44 shows why iteration 3200 iterations (black line) of the 3D ML-EM positron range correction algorithm yields the closest match of the $7 \mathrm{~T}$ (axial) PSF to the $0 \mathrm{~T}$ PSF. . . . 219

7.44 Zoomed in images on the peaks of the $\mathrm{x}$ projection from $7 \mathrm{~T}$ (3 orientations) and $7 \mathrm{~T}$ (axial) PSFs. The purpose of this plot is to show that 3600 and 3200 iterations (black line) of the 3D ML-EM positron range correction algorithm yields the closest match of the $7 \mathrm{~T}$ (3 orientation) PSF to the 0 T PSF. . . . . . . . . . . . . . . . . . 219 
7.45 Projections through the maximum of the PSFs along the $\mathrm{x}$ and $\mathrm{z}$ directions of a 0 T PSF (iteration 6000) and a $7 \mathrm{~T}$ three B-field orientation PSF (iteration 3600) using the 3D ML-EM positron range correction algorithm. The difference between these two point spread functions is small, and can thus be used as a better estimate of the difference in the resolution-noise curves between the two data types. Table 7.2 contains the FWHM and FWTM values for the $\mathrm{x}$ and $\mathrm{z}$ projections. . . . . . . 220

7.46 Reconstructed 0 T, $7 \mathrm{~T}$ (3 orientations), and $7 \mathrm{~T}$ (axial) PSFs reconstructed to iterations 6000,3600 , and 3200, respectively. The purpose of the plot is to shown that the difference discussed between the PSF is relatively small when compared to the entire FOV. . . . . . . . . . 222

7.47 Zoomed in images of the reconstructed $0 \mathrm{~T}, 7 \mathrm{~T}$ (3 orientations), and 7 T (axial) PSFs reconstructed with iterations 6000, 3600, and 3200, respectively. . . . . . . . . . . . . . . . . . 223

7.48 Left: the image of the difference between the $7 \mathrm{~T}$ (3 orientations) PSF and the 0 T PSF shown in Fig. 7.46. Right: the image of the difference between the $7 \mathrm{~T}$ (axial) PSF and the $0 \mathrm{~T}$ PSF shown in Fig. 7.46. . . 224

7.49 Difference plot for $0 \mathrm{~T}$ and $7 \mathrm{~T}$ small perturbative source in a constant background yz plane. The sum of the voxel content (in 3D) sums to 1.0 for both $0 \mathrm{~T}$ and $7 \mathrm{~T}$. Note that the positron range correction is having difficulty in this environment. In particular note the crossing artifact in the $7 \mathrm{~T}$ data suggesting that the algorithm is slow to remove the positron range. . . . . . . . . . . . . . . 225 
7.50 Left: the image of the difference between the $7 \mathrm{~T}$ (3 orientations) PSF and the 0 T PSF shown in Fig. 7.49. Right: the image of the difference between the $7 \mathrm{~T}$ (axial) PSF and the $0 \mathrm{~T}$ PSF shown in Fig. 7.46. . . 226

7.51 Images of the central slice of a uniform background using $10^{8}$ Poisson distributed events reconstructed for $0 \mathrm{~T}$ and $7 \mathrm{~T}$ data in three orientations. For the $0 \mathrm{~T}$ data 6000 iterations were used, and 3600 iterations for the $7 \mathrm{~T}$ (3 orientations) data. . . . . . . . . . . . 226

7.52 Example of the x-projection through the central maximum of the $0 \mathrm{~T}$ PSF without any system blurring (blue) and blurred with either a 0.4 mm (red) or $2.0 \mathrm{~mm}$ (black) FWHM 3D Gaussian. . . . . . . . . . . 228

7.53 Bar graph of the voxel intensities blurred for a $0.4 \mathrm{~mm}$ FWHM Gaussian system resolution for the $0 \mathrm{~T}$ and $7 \mathrm{~T}$ (3 orientation) for $253 \mathrm{D}$ ML-EM reconstructions realizations used to calculate the first standard deviation in the noise value. . . . . . . . . . . . . .

7.54 Bar graph of the voxel intensities blurred for a $2.0 \mathrm{~mm}$ FWHM Gaussian system resolution for the $0 \mathrm{~T}$ and $7 \mathrm{~T}$ (3 orientation) 3D ML-EM reconstructions realizations used to calculate the first standard deviation in the noise value. . . . . . . . . . . . . . . . . . . . . . . . . . 229

7.55 Plot of the standard deviation of the noise versus FWHM resolution in the x-direction. The error bars represent the uncertainty of the standard deviation. For 25 realization the uncertainty for every noise point is 14.43 percent. . . . . . . . . . . . . . . . . 231 
7.56 Plot of the standard deviation of the noise versus FWHM resolution in the z-direction. The error bars represent the uncertainty of the standard deviation. For 25 realization the uncertainty for every noise point is 14.43 percent. . . . . . . . . . . . . . . . . . . 232

7.57 Relative advantage . . . . . . . . . . . . . . . 234 


\section{CHAPTER 1}

\section{An Introduction to Positron Emission Tomography}

\subsection{The Basics of the PET Principle}

Positron Emission Tomography (PET) is a non-invasive medical imaging technique that is used to create images of metabolic processes in the body, and is thus an important tool for both clinical and research applications. PET imaging relies on a radioactive tracer that is inserted into the body attached to common molecules that the body uses to function, such as glucose. The basic physics principles used in PET imaging are illustrated in Fig. 1.1. The radioactive tracer emits a positron as it decays into a lower energy state. This positron is ejected from the nucleus of the atom and scatters through the tissue of the patient. With each scattering interaction the positron losses kinetic energy and eventually annihilates with an electron already present in the patient's body. Both energy and momentum must be conserved in the positron-electron annihilation. As a result, two $511 \mathrm{keV}$ gamma rays are emitted at nearly 180 degrees relative to each other ${ }^{1}$. The gamma rays are sufficiently energetic that typically around 40 percent of the photon pairs will penetrate through the body

${ }^{1}$ Slight deviations in the $511 \mathrm{keV}$ gamma ray energies and the 180 degree scattering angle will occur for positron-electron annihilations that occur when the momentum of the center-of-mass of the system is non-zero. 


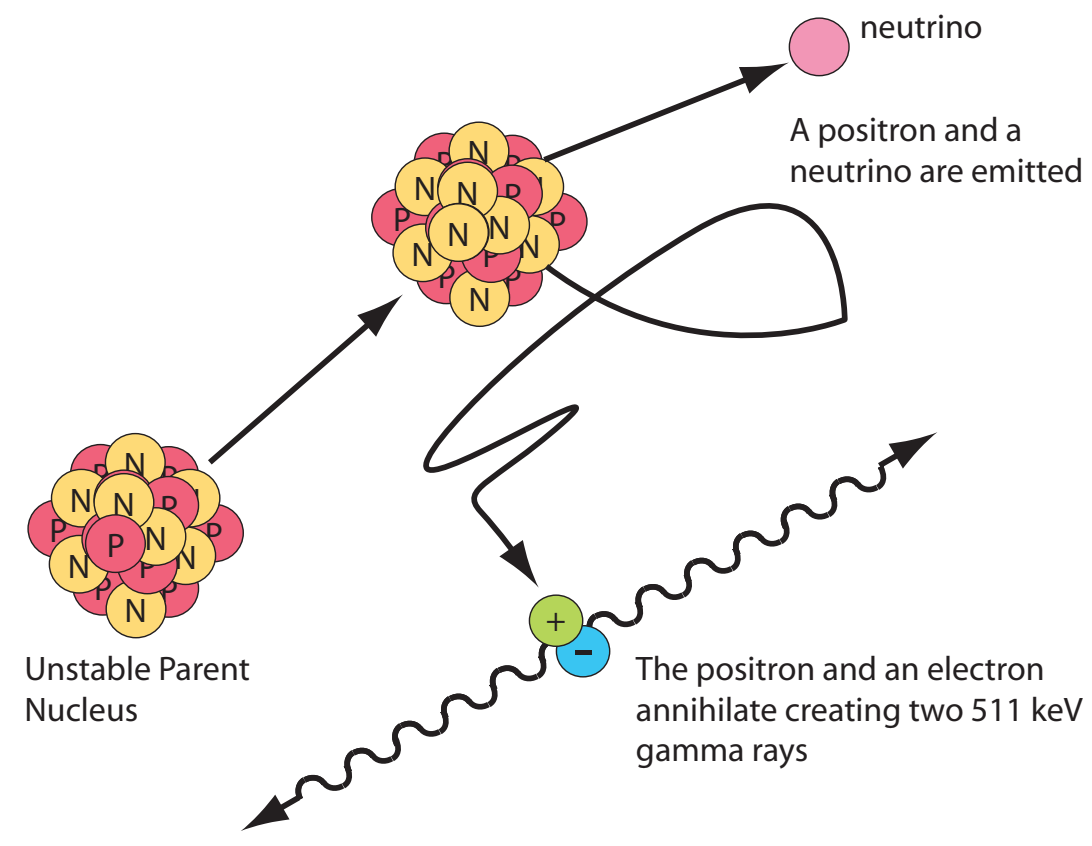

Figure 1.1: An illustration of the basic physics principles of PET imaging.

undeflected $^{2}$. A ring of photon detectors is set up around the patient to detect the positions and energies of the annihilation photons (Fig. 1.2). Timing electronics are used to determine if the photons arrive within a sensitive timing window. If so, then the energy and position of the two photons are recorded. It is then known that the annihilation event occurred somewhere along the line joining the two detected photons. By taking a large enough data sample, many lines can be back-projected between all of the detected photon pairs. A reconstruction algorithm is applied to this data to produce an image of the radio-active tracer concentration.

${ }^{2}$ This value was calculated using a $10 \mathrm{mCi}$ cylindrical line source $20 \mathrm{~cm}$ in diameter and $70 \mathrm{~cm}$ in length according to the 2001 National Electrical Manufacturers Association (NEMA) protocol collected from a clinical full body BGO scanner with a lower energy threshold of $375 \mathrm{keV}$ [1]. 


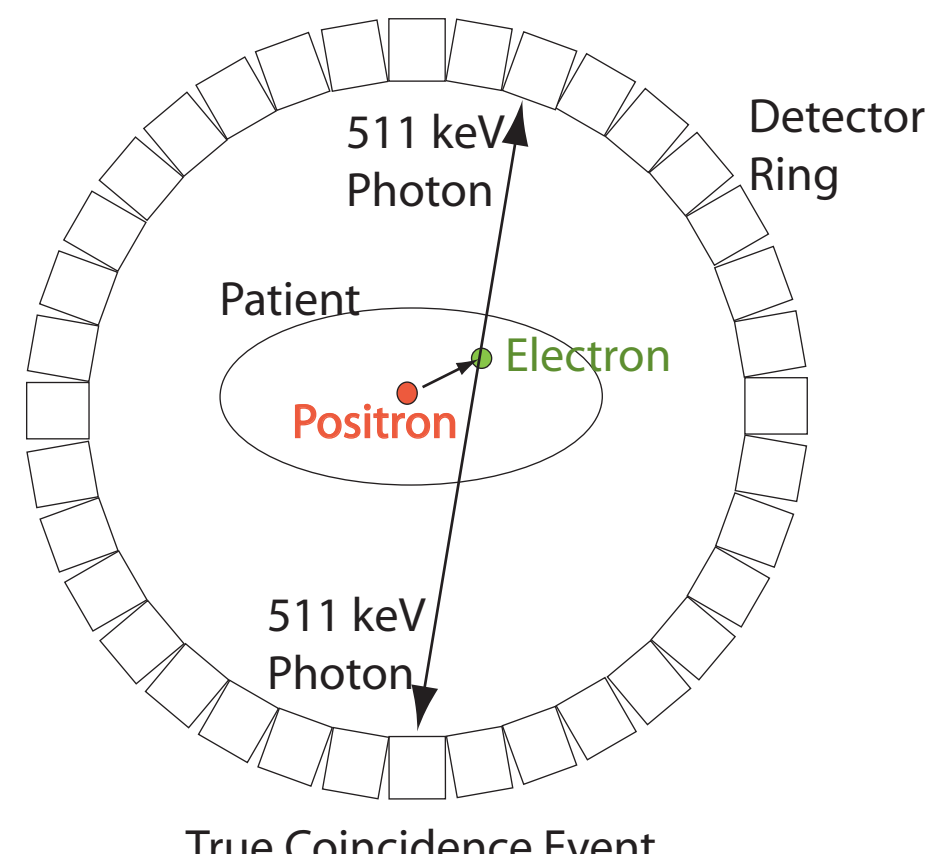

True Coincidence Event

Figure 1.2: The basic principle of PET detection.

PET imaging is useful because the PET tracers are used by the body as if they were the natural, unmodified molecules. Fluorodeoxyglucose (FDG), a glucose molecule with a ${ }^{18} \mathrm{~F}$ atom attached, is currently the most widely used radioactive tracer employed in clinical PET scanners. The advantage to using FDG is that cancer cells in the body have a higher metabolism than normal, healthy cells. As a consequence, cancer cells will metabolize more FDG, thus accumulating a larger concentration of the radiotracer than the surrounding normal, healthy cells. The role of PET systems is to create images of the distribution and concentration of the radiotracer in the patient.

There are three types of coincidence events that are detected by PET scanners: true, scattered, and random events (see Fig. 1.3). True coincidence events occur 


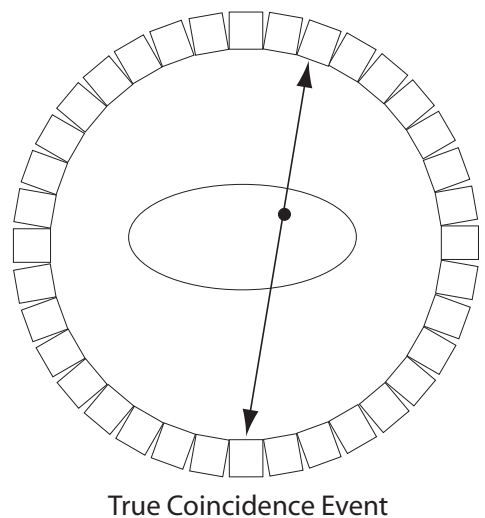

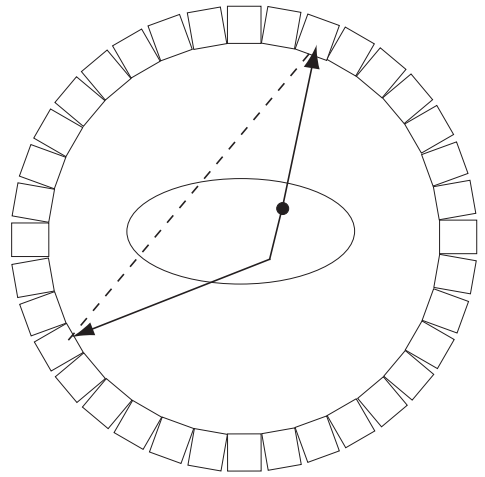

Scattered Coincidence Event

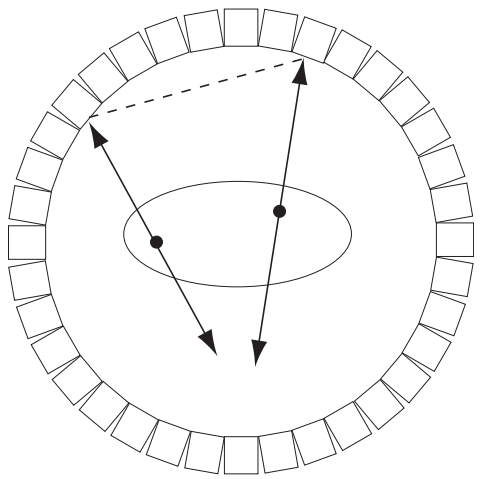

Random Coincidence Event

Figure 1.3: The three types of PET coincidence events: true, scattered, and random coincidence events. The dotted line in the figures represent the back-projected lineof-response (LOR)

when the two emitted gamma rays pass through the patient and the air undeflected and are detected by opposing detector elements. Neglecting uncertainties of the detection process that will be discussed shortly, the line-of-response (LOR) that is back-projected through the image space will pass through the annihilation point for true coincidence events. Scattered coincidence events occur when one or both of the gamma rays is scattered off of an atom in the patient or surrounding air prior to being detected by the PET ring. In this case, the two photons will still arrive within the coincidence timing window, but the LOR joining the two detectors will not pass through the annihilation point. The scattered photon's energy will be less than 511 $\mathrm{keV}$. Depending on the energy resolution of the photon detector, an energy window can be used that will only record events that have photon energies peaked around $511 \mathrm{keV}$. However, this only works if all of the energy of the photon is absorbed in the PET detector. Higher energy resolution PET detectors can have finer energy cuts that require the detected photon energy to be tightly peaked around $511 \mathrm{keV}$, 
thus resulting in a reduced scattered coincidence rate. Recording scattered events introduces background and statistical noise into the reconstruction process and can degrade the image quality. The final type of events detected by PET systems are random events which occur when two independent annihilation events occur simultaneously and two photons from the opposing pairs are accidently collected within the coincidence timing window. The likelihood of detecting random events increases with high activity tracer doses and for wider coincidence timing windows. State-of-the-art PET coincidence timing windows are on the order of a 200 ps - 2 ns [2].

\section{$1.2 \quad$ PET Design Considerations}

There are two important quantities to consider when designing a PET system: image resolution and sensitivity. The image resolution is a measure of the level of detail that can be distinguished in the images, while the sensitivity refers to the ratio of the number of coincidence photons detected compared to the total number of annihilation events. The ideal PET scanner would possess both excellent image resolution and high sensitivity. When using PET scanners to detect cancer, higher resolution images translate into earlier detection and treatment of the disease which ultimately leads to an increased likelihood of recovery. High sensitivity is important in reducing the scan times which reduces the likelihood of blurring due to patient movement. More efficient PET system also allow lower activity doses of radiation to be administered to the patient, minimizing the patient's radiation exposure.

The following list briefly summarizes the factors that contribute to the image resolution and sensitivity of PET scanners. 


\subsubsection{Spatial resolution and sensitivity of the photon detec- tors}

Historically, the image resolution of PET scanners has been limited by the ability to localize the photon interaction positions within the PET detector ring. By minimizing the uncertainty of the photon interaction positions, finer LORs can be back-projected into the image space resulting in the potential for higher resolution images. Thus, it has been the trend to use finer and finer detector elements in order to reduce the uncertainty of the photon interaction positions.

The two main draw backs of using finer detector elements include increased cost and complexity due to the larger number of readout channels and the possibility of depth of interaction errors which occur when photons enter the detector at an angle and subsequently penetrate a few neighboring detector elements prior to interacting. As shown in Fig. 1.4, the back-projected LOR will not pass through the annihilation location and will ultimately lead to poorer system resolutions.

PET systems have traditionally been designed using scintillator crystals coupled to an array of photo-multiplier tubes (PMTs). In these systems the incident $511 \mathrm{keV}$ gamma rays interact with the scintillator by either Compton scattering off of atomically bound electrons or by photo-electric absorption. The energy liberated by the incident photon is transformed into flashes of light that illuminate the PMTs. The scintillation light is sufficiently energetic that photo-electrons are created when it strikes the first photo-cathode of the PMT. An electric field guides the liberated electrons to anther electrode resulting in additional electrons. This process is continued ultimately resulting in a surge of current proportional to the energy deposited by the incident gamma ray photon. 


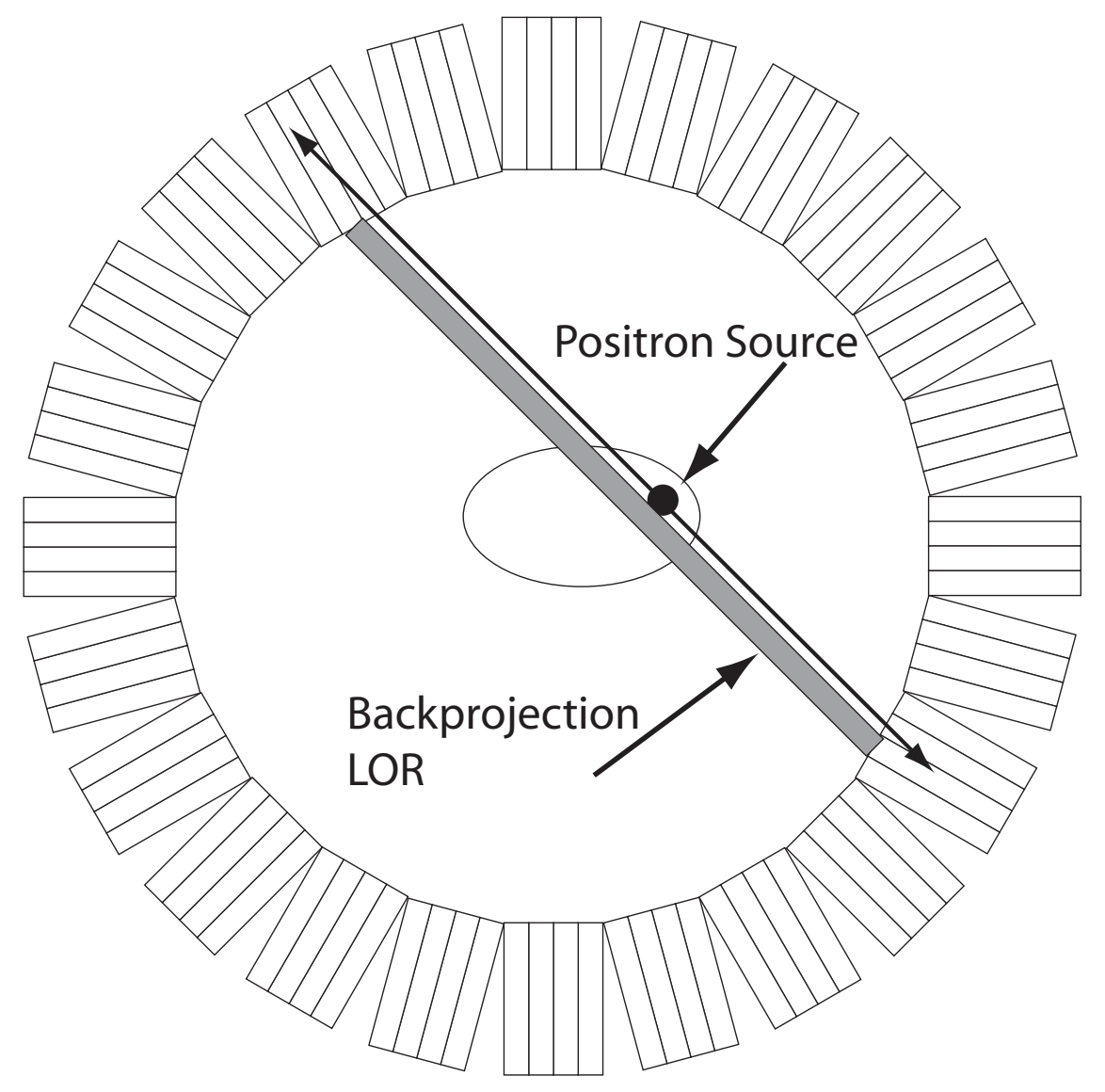

Figure 1.4: Illustration of positioning errors that can occur when photons penetrate PET crystals at an angle relative to the face of the detector and penetration into neighboring detector elements prior to interacting. This depth of interaction results in back-projected LORs that do not pass through the positron-electron annihilation position. 


\begin{tabular}{|c|c|c|c|c|c|c|}
\hline Property & LSO & BGO & $\mathrm{NaI}(\mathrm{Tl})$ & $\mathrm{Si}$ & $\mathrm{Ge}$ & $\mathrm{CZT}$ \\
\hline Effective atomic number & 66 & 75 & 51 & 14 & 32 & 50 \\
\hline Density $\left(\mathrm{g} \mathrm{cm}^{-3}\right)$ & 7.40 & 7.13 & 3.67 & 2.33 & 5.32 & 5.80 \\
\hline $\begin{array}{l}\text { Linear attenuation coefficient } \\
\left(\mathrm{cm}^{-1}\right)\end{array}$ & 0.87 & 0.96 & 0.34 & 0.20 & 0.43 & 0.53 \\
\hline Photoelectric fraction & 0.32 & 0.41 & 0.17 & 0.002 & 0.04 & 0.17 \\
\hline Compton fraction & 0.63 & 0.54 & 0.79 & 0.99 & 0.93 & 0.79 \\
\hline $\begin{array}{l}\text { Mean number of electrons } \\
\text { created per } \mathrm{MeV}^{*}\end{array}$ & 4,400 & 1,200 & 7600 & $2.8 \times 10^{5}$ & $3.4 \times 10^{5}$ & $2 \times 10^{5}$ \\
\hline
\end{tabular}

Table 1.1: Physical properties of detector materials used in PET. *For LSO, BGO, and $\mathrm{NaI}(\mathrm{Tl})$ a 20 percent photo-multiplier tube (PMT) quantum efficiency is assumed $[4][5][6]$.

An advantage of scintillator PET systems is their high stopping power due to their large effective atomic number Z (see Table 1.1). This high stopping power translates into better system efficiencies. However, the large stopping power also translates into an increased probability of multiple scattering events which degrades the spatial resolution of the detector. One technique for improving the spatial resolution of scintillator/PMT PET systems is to construct an array of fine scintillation crystals elements. The ideal dimensions of an individual scintillation crystal used in the array is to possess a small area face incident to the annihilation radiation (for improved spatial resolution) and to be long and tightly packed (such that sensitivity is not lost) $[3]$.

Solid state detectors can also be used to construct PET systems. ${ }^{3}$ The main advantage of using solid state detectors in PET is that the detection elements (pixels)

\footnotetext{
${ }^{3}$ The physics of solid state detectors is discussed in chapter 3 .
} 
can be made relatively small (on the order of a $1.0 \mathrm{~mm}^{2}$ or less). As previously discussed, small detection elements will yield higher resolution images because finer LORs can be back-projected into the image space. Solid state detectors also posses excellent energy resolutions because they directly collect the charge carriers liberated by the incident radiation whereas scintillators have the complication of collecting the emitted light through photomultipliers. Due to the collection technique and the larger number of free charge carriers created in semiconductors compared to scinitllator/PMT systems (see Table 1.1) the statistical fluctuations will be smaller for large number of charge carriers, thus leading to superior energy resolutions. For identical detector volumes, scintillators typically have a higher efficiency than semiconductor detectors due to their larger effective atomic number Z (with the exception of CZT). However, the spatial resolution is typically poorer in scinitillators due to the increased probability of multiple scattering events due to their higher atomic numbers and densities. Also, depth of interaction effects can degrade the spatial resolution for finely segmented scintillation crystals when annihilation photons enter at an angle relative to the normal vector defined by the detector face (solid state pad detectors do not have this problem due the geometry of the detection elements). Additional disadvantages of semiconductors include a limitation on a single crystal's thickness (which can be solved with a stack of thin detectors at the expense of increased readout complexity) and difficulty with achieving adequate timing resolutions [7] [8] [9].

\subsubsection{Positron Range}

In PET imaging it is desired to reconstruct the positions of the nuclei that emit the positrons; however, the positrons are ejected into the tissue of the patient with 
nonzero kinetic energy and as a result annihilate at some distance away from their points of emission. Some tracers, such as ${ }^{18} \mathrm{~F}$, emit low energy positrons that do not penetrate far into the tissue in comparison to the image resolution of most PET scanner. These positron emitters will not seriously degrade the image resolution; however, higher energy positron emitters such as ${ }^{68} \mathrm{Ga}$ and ${ }^{94 m} \mathrm{Tc}$ emit positrons that can penetrate several millimeters before annihilating. These higher energy radioisotopes will seriously degrade PET scanners with resolutions in the $1 \mathrm{~mm}$ regime, such as state-of-the-art small animal PET scanners [7] [10].

The work contained in this document investigates techniques that reduce the effects the positron range have on the image resolution of PET scanners.

\subsubsection{Doppler broadening}

The electrons bound to the atoms in the patient possess a non-zero momentum. Both momentum and energy must be conserved in the annihilation event between the positron and the atomically bound electron. As a result, the photons are not emitted exactly at 180 degrees with respect to one another as they would be if the positron-electron system annihilated at rest. Instead there is a small deviation from the ideal 180 degree emission angle. This small angular deviation is referred to as the acollinearity angle. For annihilation events in water, the acollinearity angle is a Gaussian distribution that has been measured to be $0.5 \mathrm{~mm}$ FWHM [11]. This effect is known as Doppler broadening. Figure 1.5 demonstrates that the image blur due to the acollinearity angle will become more severe as the inner diameter of the PET ring is increased. 


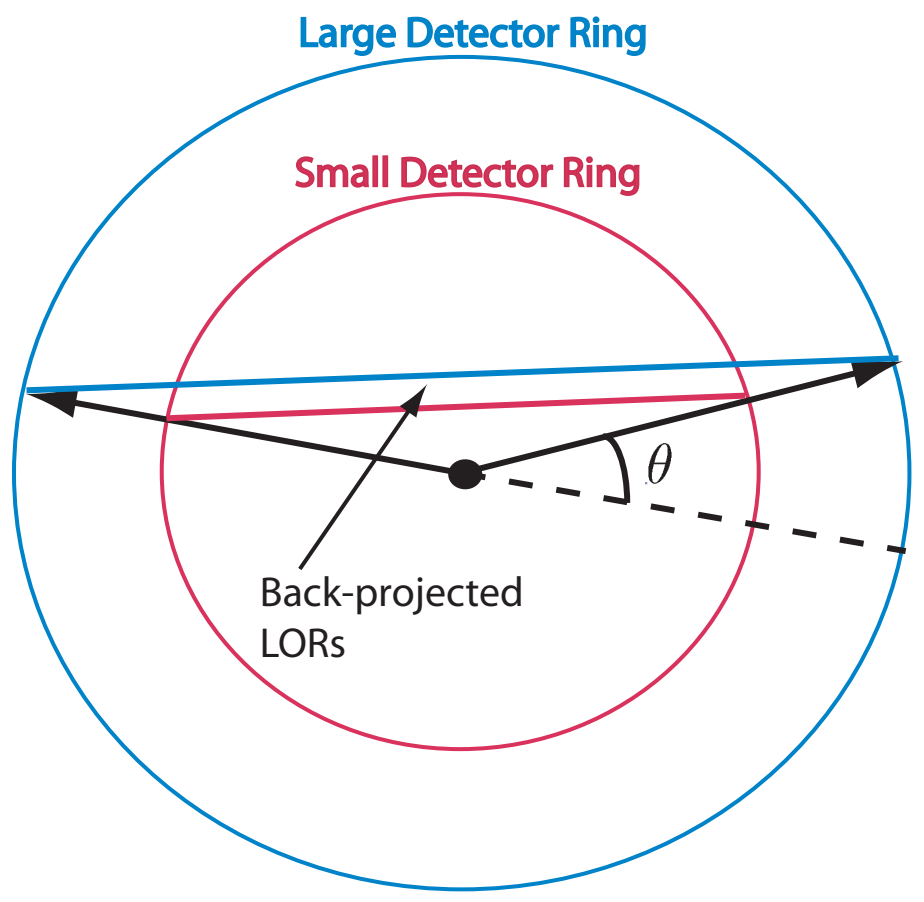

Figure 1.5: Illustration of the error on the back-projected LORs due to photon acollinearity and the diameter of the PET detection ring. 
It should also be noted that the change in energy of the annihilation photons due to the kinetic energy of the center-of-mass of the system is negligible in comparison to the energy resolution of current state-of-the-art detectors used in PET. For example, using a 0.5 degree acollinearity angle will yield a deviation of $0.02 \mathrm{keV}$ in the energy of an annihilation photon. Since the best energy resolution of current PET detectors are approaching $1.0 \mathrm{keV}$, this shift in the annihilation photon energy cannot be resolved and is neglected.

\subsubsection{Timing resolution}

The timing resolution is important in order to reduce random coincidence events that degrade the image quality for PET scanners imaging high activity sources. The timing resolution is extremely important for clinical applications in order to collect an adequate data sample in as short a time as possible. Shorter scan times will reduce the chance of image blur due to patient movement and will also reduce the stress on the patient due to the confinement of the PET imaging process. PET systems with poor timing resolutions must use wider coincidence windows such that the sensitivity is not sacrificed. Large coincidence windows lead to increased random coincidence rates for a fixed source activity. Thus, excellent timing resolution (on the order of $5 \mathrm{~ns}$ or less for a $200 \mathrm{MHz}$ PET systems) is desired to reduce random coincidences that degrade the image quality.

Fast timing resolution can also be used to make time-of-flight corrections to the LORs. For fine enough timing resolution, the time difference between the two detected coincidence photons can be determined. Using this time difference and the known speed of the photons, the back-projected LOR can be weighted based on the 
probability that the photon pair originated from a restricted region of the LOR. The fastest time-of-flight PET systems have coincidence timing resolutions on the order of $100 \mathrm{ps}$, which translates into a position uncertainty of $30 \mathrm{~mm}$ [2]. Smaller diameter detector rings demand even finer timing resolution due to the shorter distance the photons must travel before reaching the detectors. The coincidence timing used in this experiment is far too course to employ time of flight corrections and will not be discussed further.

\subsubsection{System Dead Time}

Dead time is the amount of time that the system takes to process an event. During this time, no additional events can be detected. Fast readout electronics is important for reducing the dead-time of the readout cycle. The time it takes to process and readout an event should be as short as possible. Fast readout times translate into less dead-time and higher event collection rates, i.e. better sensitivity. This has direct implications on the radiation dose that can be administered to the patient. Fast readout electronics reduce the dead-time and allows for a lower does of radiation to be administered to the patient without sacrificing image quality.

\subsubsection{Sensitivity Considerations}

The sensitivity of PET scanners is important in order to collect a large enough data sample to adequately reconstruct the data in as short a time as possible (the average clinical PET scan takes approximately 30 minutes). High sensitivities directly reduce the amount of radiation that needs to be administered to a patient without sacrificing image quality. Techniques to improve sensitivity include: using photon

detectors with high stopping power (detect as many gamma ray photons as possible), 
increasing the area of PET detectors surrounding the patient in order to cover a larger solid angle (increase the length of the cylindrical volume for 3D PET), fast coincidence timing with fast readout electronics (less dead time equates to a lower radiation dose).

\subsection{Applications of PET Technology}

PET technology is an important tool for the diagnosis and treatment of disease in humans [12] [13] [14]; however, other applications exist for PET systems. One area of study that has been the focus of many research groups is the development of PET systems for use in imaging small animals [15] [16] [17]. Dedicated small animal PET systems are important for biomedical, pharmaceutical, and genetic manipulation applications. One prominent example is in the use of animals as human disease models. Mice have become the choice animal for modeling and testing treatments on human diseases due to the similarity between our genetic codes (most human genes have a related mouse gene) [18]. Geneticists now have the ability to knock out and replace genes suspected to cause disease. These genetically altered mice have offspring, thus passing down their genetically altered state. Conventionally, the disease studies done on these mice demand sacrificing large number of the offsprings at various stages of disease development or treatment. These studies can become expensive due to the number of mice needed. Adding to the equation genetically altered mice, the cost can quickly escalate to over a thousand dollars per mouse [1]. Hence the ability to study the same mouse through the entire development and treatment of a disease without sacrificing the animal would be preferable. A small animal PET system allows for this opportunity. 
There are challenges that must be overcome to convert the technology developed for conventional PET into the small animal domain. One of the main issue that needs to be addressed is the spatial resolution. The spatial resolution needs to be improved over conventional PET due to the large difference in the volume being imaged between human full body scans and mice, which represents about a 200 fold decrease in volume. In order to achieve the same image quality seen in conventional, human imaging PET, the resolution to volume ratio must be the same for both imaging modalities. This corresponds to an order of magnitude improvement in resolution for small animal PET systems over full-body human PET systems. A typical PET system has a resolution of approximately $10 \mathrm{~mm}$ for full human body scans, thus for the small animal set-up to have the same image quality a resolution of less than $1 \mathrm{~mm}$ needs to be achieved $[1]$.

One may think that the efficiency when imaging small animals may not be as large a problem as when imaging humans because there are no radiation exposure limits for animals. This is not the case. One of the fundamental principals of tracer kinetic experiments is that the mass levels of the tracer can not interfere with normal biological functions. Many circumstances exist in which the mass of the tracer will limit the amount of radioactivity that can be injected into the animal, thus an increase in efficiency over conventional PET is desirable. For full body human scans the absolute efficiencies of PET detectors are in the range of $0.3-0.6$ percent for $2 \mathrm{D}$ acquisition and 2-4 percent for 3D acquisition [1]. To achieve the same sensitivity to volume ratio between human and small animal PET scanners a three order of magnitude increase in sensitivity is needed. Unfortunately, this is not possible even with 100 percent efficient detectors and full angular coverage. The best that can be 
achieved is a 200 fold increase in sensitivity in 2D mode and a 30 fold increase in 3D mode [19]. These numbers are not as bad as it may seem. Small animal PET has an important advantage over conventional PET in that the amount of material the photon pairs must pass through is much less for small animals than for humans. Therefore, significantly less photons will scatter within the patient leading to a much higher ratio of true coincidence events to scattered coincidence events. This means that less events need to be collected if more of the events are true coincidences. Iterative reconstruction techniques that model the physics of PET systems also relax the demand on the sensitivity of the small animal PET system in contrast to using analytical reconstruction techniques such as filtered back projection (FBP).

\subsection{Thesis Overview: Techniques to reduce the image blur due to the positron range}

With the image resolution of state-of-the-art small animal PET systems in the sub-millimeter regime, the most dominate factor influencing image blur is due to the displacement of the positron from its parent nucleus to its annihilation position. For true coincidence events the back-projected LORs between two detected coincidence photons will pass through the positron-electron annihilation point. However, the desired distribution to be imaged is the location of the parent nucleus that emitted the positron, not the annihilation position. For low energy positron emitters the annihilation position and the location of the parent nucleus are nearly indistinguishable for current resolution PET systems because the FWHM range of commonly used ${ }^{18} \mathrm{~F}$ is approximately $0.1 \mathrm{~mm}$, well below the $1.0 \mathrm{~mm}$ FWHM resolution of state-of-the-art PET scanners. However, for higher energy positron emitters, such as ${ }^{68} \mathrm{Ga}$ and ${ }^{94 m} \mathrm{Tc}$, 
the average range of the positron is on the order of millimeters and is the dominate factor contributing to the final image resolution.

It has been suggested by Raylman, Hammer, and Christensen to embed the field of view of a PET scanner in a strong magnetic field in order to reduce the average positron range [20]. The positrons will experience a Lorentz force as they travel through a magnetic field. This force will cause the positrons to spiral in the plane transverse to the applied magnetic field; however, the component of the positron's velocity aligned with the magnetic field vector will not be affected. In the absence of a scattering medium, the path of a positron within a static, homogeneous magnetic field will trace out a helix. However, in PET applications the scattering medium is the tissue of the patient, and consequently, the positron will follow a tortuous path as it scatters off of the atoms in the patient, losing energy with each interaction. The net result of the applied magnetic field is to curve the positron between physics interactions, thus reducing the final displacement of the positron annihilation position from its parent nucleus. The displacement of the positron from its point of emission, on average, will be reduced in strong magnetic fields allowing for the potential of higher resolution images.

The image resolution of PET scanners can also be improved using a three dimensional maximum likelihood expectation-maximization reconstruction algorithm (ML-EM) that statistically corrects for the positron range distribution; however, the improvement in image resolution is coupled to an increase in noise due to the Poisson nature of the data. The ultimate purpose of this study is to compare the trade-off between the resolution and the noise using a 3D ML-EM algorithm that statistically corrects for the positron range at $0 \mathrm{~T}$ and in strong magnetic fields. 
This dissertation investigates the effects strong magnetic fields and iterative reconstruction techniques that statistically correct for the range of the positron have on the image quality of PET scanners. The culmination of this thesis is a study that examines the resolution-noise properties of PET systems utilizing both strong magnetic fields and an iterative reconstruction algorithm that statistically corrects for the range of the positron.

The structure of this thesis is as follows. Chapter 2 explains the physics relevant to the PET imaging process along with the simulation techniques that model the physics followed by the relevant simulation results. In this study, solid state silicon detectors are used to detect the annihilation photons. Chapter 3 describes the physics of silicon detectors, and the details and performance characteristics of the silicon detectors used in this study. Chapter 4 describes the small animal PET prototype that is used to measure the reduction of the positron range in strong magnetic fields. Chapter 5 describes the $2 \mathrm{D}$ and $3 \mathrm{D}$ iterative reconstruction techniques. Chapter 6 presents the measured, experimental results of the positron range at $0 \mathrm{~T}$ and transverse to a 7 T magnetic field for both low and high energy positron emitting point sources. The results are compared to the simulations detailed in chapter 2. Finally, chapter 7 investigates the improvement in image resolution using the 3D ML-EM positron range correction algorithm for simple and complex source distributions. The second half of chapter 7 compares the resolution-noise properties of systems using both strong magnetic fields and iterative reconstruction algorithms that statistically corrected for the positron range. 


\section{CHAPTER 2}

\section{PET Physics and Simulations}

\section{$2.1 \quad$ Overview}

This chapter addresses the physics relevant to PET imaging along with the Electron Gamma Shower simulation code (EGS4) that models the physics interactions between electrons, positron, and photons as they traverse through matter. The topics covered in this chapter include the nuclear decay of exited atoms that result in the emission of a positron, the interactions of positrons as they scatters through matter, the physics of positron-electron annihilation, and the interaction of photons with matter. This chapter is structured into sections that describe first the physics, followed by the EGS4 simulation techniques and simulation results for each set of physical interactions. The next section gives an overview of the EGS4 simulation package.

\subsection{The Electron Gamma Shower Simulation Package (EGS4)}

Electron Gamma Shower (EGS4) is a Monte Carlo simulation code that tracks the transport of electrons, positions, and photons through matter in the energy range of $1 \mathrm{keV}$ through $2.0 \mathrm{TeV}$ for photons and $10 \mathrm{keV}$ through $2.0 \mathrm{TeV}$ for electrons and positrons taking into account all of the relevant physics interactions (with the 
exception of Doppler broadening). The following physics interactions are included in the EGS4 simulation:

- Bremsstrahlung - Occurs when a high energy electron is deflected by the electric field of a charge distribution such as the nucleus of the atom.

- Positron-electron annihilation - Occurs when a positron and electron come into contact and are replaced by two nearly collinear $511 \mathrm{keV}$ gamma rays.

- Coulomb scattering of electrons and positrons off of atomic nuclei.

- Molière's scattering - the cumulative scattering angle of beta particles (electrons and positrons) traveling through a thickness of material resulting in the deflection of the incident particle from its original trajectory due to the elastic Coulomb scattering with the local atomic nuclei.

- Bhabha scattering - the inelastic scattering of an electron and a positron.

- Continuous energy loss of charged particles by both Bremsstrahlung and a collisional loss terms determined by the Bethe-Block equation.

- Pair Production - the inverse physical process of positron-electron annihilation. This process can only occur for photons with energies greater or equal to 1022 $\mathrm{MeV}$ (the combined rest mass of the positron and electron) and in the presence of an atomic nuclei in order to conserve both energy and momentum.

- Compton scattering - The scattering of a photon off of an electron where a portion of the photon's energy and momentum are transferring to the electron.

- coherent Rayleigh scattering 
- Photo-Electric absorption - occurs when a photon transfers all of its energy to an atomic electron, ejecting it from the atom. An electron from a higher energy shell will transition into the vacancy left by the excited electron resulting in the emission of a photon. The energy of the photon is simply the energy difference between the two electron orbital states.

It is important to note that Doppler broadening, an important physical effect that degrades PET image resolution, is not included in the EGS4 simulation package. However, it is still included in this study. Doppler broadening is added "by-hand" between key EGS4 simulation steps. The details of this operation are described in section 2.6.

In order to properly simulate the path of electrons, positrons, and photons through matter, the EGS4 code must know the cross sections for all of the physical interactions that each particle type will experience as it scatters through the media. The cross sectional data used in the EGS4 code are created by the PEGS4 (pre-EGS4) program [21]. PEGS4 creates cross sectional data based on user specified inputs such as material composition (element, mixture, or compound) density, electron and photon lower threshold cut-off energies, and an optional flag to include ICRU (International Commission on Radiation Units) density corrections. The ICRU density correction file for liquid water is used in this study with lower threshold energy cuts of $10 \mathrm{keV}$ for both electron and positron transport and $1 \mathrm{keV}$ for photon transport.

The EGS4 code is written in Mortran3, a Fortran 77 based macro language [21]. The EGS4 code is split into two sections: user written code and EGS code. The user written code controls the initial conditions of the incident particle (particle type, initial kinetic energy, velocity, position), the HOWFAR routine which describes the 


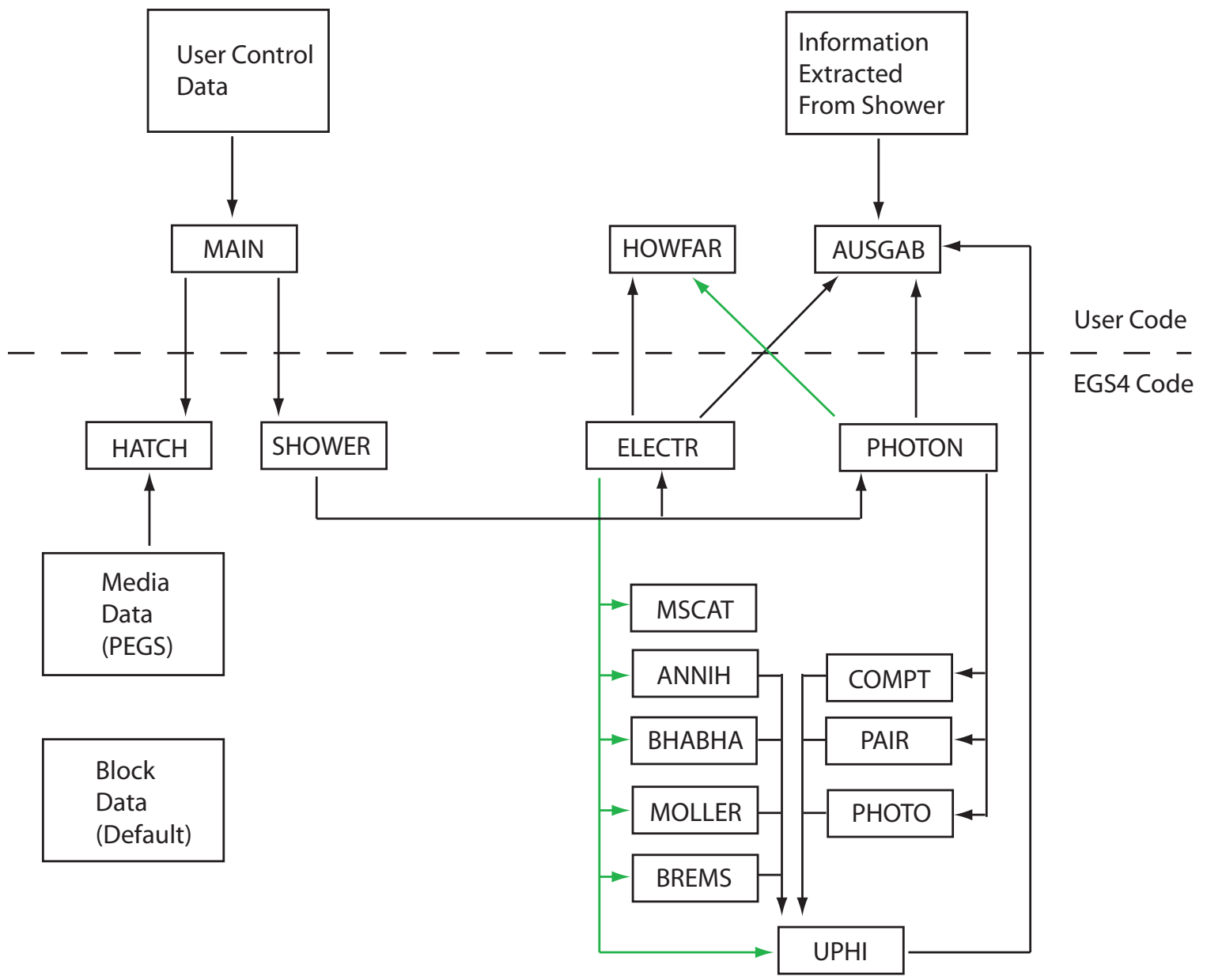

Figure 2.1: Block diagram of EGS4 simulation struction.

geometry, and the AUSGAB (German for output) routine is used for outputting the desired simulation results. The EGS, behind-the-scenes, portion of the code is responsible for transporting and tracking the incident particle and all secondary particles created in the shower process.

The structure of the EGS4 code is shown in Fig. 2.1. The dashed line in the figure represents the division between user written code and the EGS4 (behind-the-scenes) code. The user written section of the code is in the MAIN program that specifies the 
initial conditions of the particles and assigns the scattering media to each region. The MAIN program also makes calls to the EGS4 HATCH and SHOWER subroutines to both set-up and execute the simulation, respectively. Also included in the user written code is the HOWFAR routine that describes the geometry of the simulation and checks to see if the simulated particles will cross a media boundary on the next simulation step. The last section of the user written code is the AUSGAB routine that outputs the results of the simulation to a standard ASCII text file. The EGS4 portion of the simulation code includes all of the physics of the simulation and is not modified by the user.

The following paragraph outlines the procedural steps of the EGS4 simulation. First, the MAIN program calls the HATCH subroutine which loads the cross-sectional data created by the PEGS4 program. EGS4 then initiates the simulation with a call to the SHOWER subroutine. The SHOWER subroutine uses the user defined initial conditions for the incident particle as inputs. Depending on the charge of the incident particle, either a call to the ELECTR or PHOTON subroutines is performed. The ELECTR subroutine is responsible for tracking charged particles through the scattering material as they continuously lose energy and determines when discrete interactions, such as an annihilation event, are to occur based on the PEGS4 cross-sections. Likewise, the PHOTON subroutine is responsible for tracking photons through matter. Unlike charged particles, photons do not continuously lose energy as they traverse through a scattering medium. Instead, photons only interact through discrete interactions such as Compton scattering, photo-absorption, and pair production. Before each step of the simulation the PHOTON subroutine determines which (if any) discrete interaction is to occur. After each call to either the ELECT or PHOTON 
subroutine is made, EGS4 consults the HOWFAR routine to determine if a boundary between geometric regions is to be crossed during the next simulation step. A call to the AUSGAB routine is made at this time where data on the current state of the particle can be written out to a file. Each particle created in the shower is followed until either its energy falls below the threshold energy or the particle enters a region that is not of interest to the user. It should also be noted that the PRESTA subroutine (parameter reduced estimated step transport algorithm) is used in this simulations. PRESTA yields improved results for particle transport across media boundary and improves tracking of low energy $\beta$ particles below $50 \mathrm{keV}[22]$.

\subsection{Simulating Positron Energy Spectrum}

PET imaging is possible due to the nuclear transmutation of unstable elements into stable elements. Examples of elements that undergo nuclear transmutation are ${ }^{18} \mathrm{~F},{ }^{11} \mathrm{C},{ }^{15} \mathrm{O},{ }^{68} \mathrm{Ga}$, and ${ }^{94 m} \mathrm{Tc}$. During the nuclear transmutation process a proton in the nucleus decays into a neutron, a positron, and an electron neutrino via the weak force. The general equation for a nuclear transmutation is given by:

$$
{ }_{Z}^{A} X \rightarrow{ }_{Z-1}^{A} Y+{ }_{1}^{0} \beta^{+}+\nu_{e}
$$

where $\mathrm{A}$ is the atomic weight, $\mathrm{Z}$ is the atomic number, $\mathrm{X}$ is the parent element, $\mathrm{Y}$ is the daughter element, $\beta^{+}$is a position, and $\nu_{e}$ is an electron neutrino. An orbital electron is also ejected from the atom in order to balance charge. The positron emitted in this reaction is the key to PET imaging.

The sum of the kinetic energy of the positron and neutrino is a constant; however, the kinetic energy is randomly divided between the two particles. The theoretical 
positron emission kinetic energy distribution is given by

$$
N(E) d E=g F(Z, E) p E\left(E_{\max }-E\right)^{2} d E
$$

where $\mathrm{N}(\mathrm{E})$ is the number of events (decays) at energy $\mathrm{E}, \mathrm{g}$ is a coupling constant (approximately $10^{-6}$ ), $E_{\max }$ is the maximum positron energy in units of $m c^{2}, \mathrm{p}$ is the momentum in units of $m c, \mathrm{~F}(\mathrm{Z}, \mathrm{E})$ is the Fermi function, and $\mathrm{Z}$ is the atomic number of the daughter atom. The Fermi function describes the interaction of the Coulomb force between the daughter nucleus and the emitted beta particle. For the case a beta decay resulting in a positron emission this force is repulsive due to the positive charge of both the nucleus and the positron. Although the emitted positrons are relativistic, the non-relativistic approximation of the Fermi function yields an excellent match to experimental data and is used to generate the positron kinetic energy spectra as shown by $\mathrm{Wu}[23]$. The non-relativistic Fermi function is given by

$$
F(Z, E)=\frac{2 \pi \eta}{1-e^{-2 \pi \eta}}
$$

where $\eta=\frac{-Z \alpha E}{p}$, where $\alpha$ is the fine structure constant $\alpha=\frac{1}{137}[23]$.

Equation 2.2 was integrated over the energy numerically in $1 \mathrm{keV}$ steps to create the positron kinetic energy spectrums for each radioisotope (Fig. 2.2). Random values consistent with the kinetic energy spectra were then generated for the isotope of interest. Plots of the number of emissions versus positron kinetic energy for commonly used radioisotope are shown in Fig. 2.3. The same number of events were generated for each positron source. A file containing the random positron emission energies is used as the input positron kinetic energy in the EGS4 simulation described in section 2.4 that tracks the positrons from their emission points to their annihilation positions. 


\begin{tabular}{lll}
\hline Isotopes & $E_{\max }(\mathrm{keV})$ & Half Life \\
\hline${ }^{22} \mathrm{Na}$ & 535 & 951 days \\
${ }^{18} \mathrm{~F}$ & 635 & $109.77 \mathrm{~min}$ \\
${ }^{11} \mathrm{C}$ & 970 & $20.39 \mathrm{~min}$ \\
${ }^{13} \mathrm{~N}$ & 1190 & $9.965 \mathrm{~min}$ \\
${ }^{15} \mathrm{O}$ & 1720 & $122.24 \mathrm{sec}$ \\
${ }^{68} \mathrm{Ga}$ & 1899 & $67.71 \mathrm{~min}$ \\
\hline
\end{tabular}

Table 2.1: Maximum positron energies and half-lives of some commonly used positron emitters.

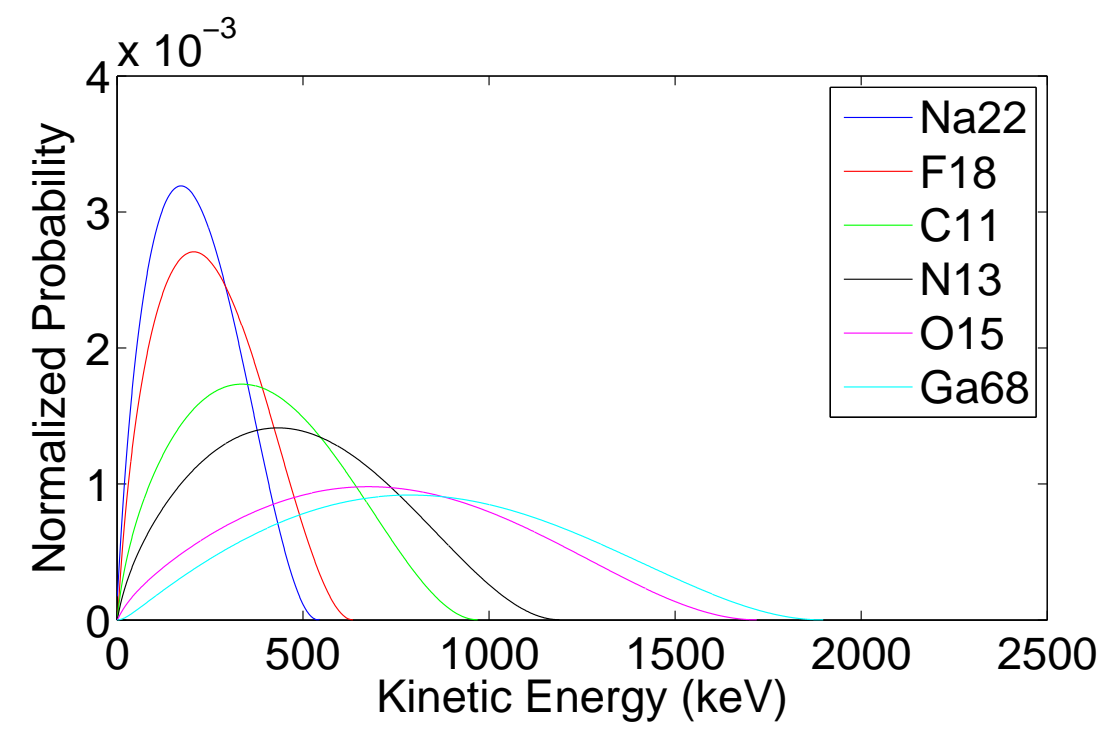

Figure 2.2: Theoretical positron kinetic energy spectrum for various radioisotopes commonly used in PET. 


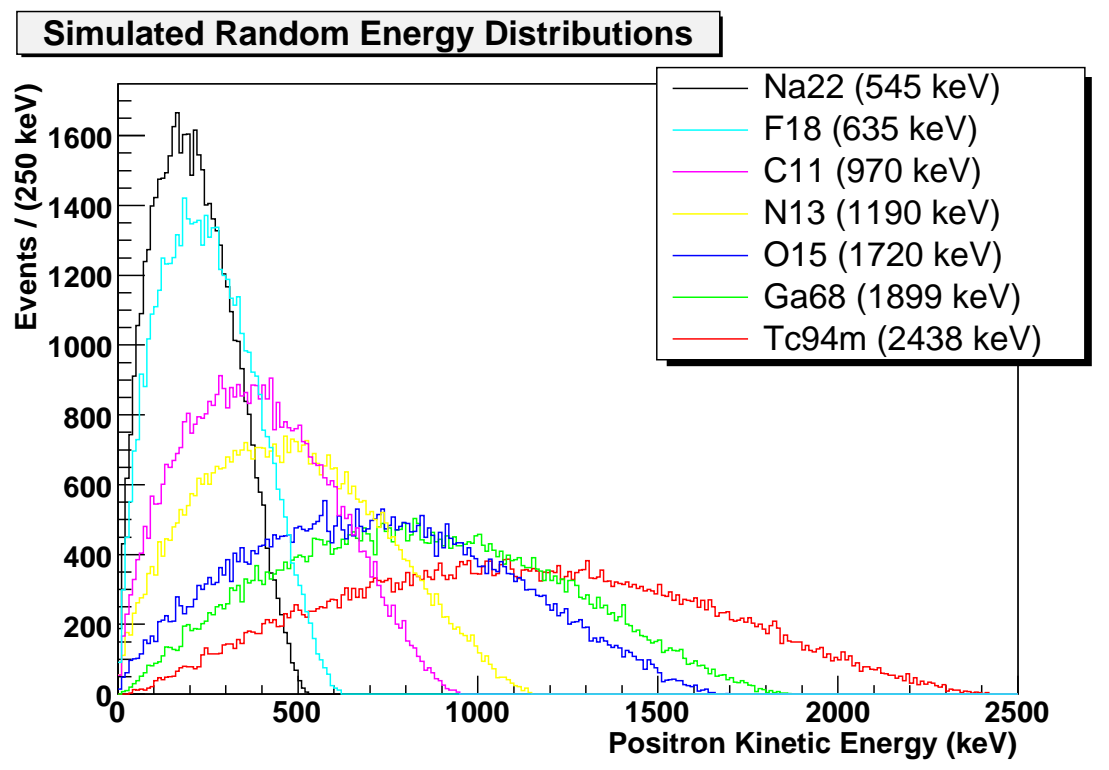

Figure 2.3: Simulated energy spectrums for positron emitting radioisotopes commonly used in PET applications. The same number of events are simulated for each distribution.

\subsection{Positron Penetration Through Matter}

The fate of the positron emitted during the nuclear decay is to eventually annihilate with an electron already present in the patient's body. However, the positronelectron annihilation will not occur immediately after the positron emission. Instead the positron will scatter off of the atoms in the patient's body losing energy with each interaction. The positron range is defined as the displacement between the emission atom and the electron-positron annihilation position. This section outlines the physical interactions positrons with energies ranging between $10 \mathrm{keV}$ and $10 \mathrm{MeV}$ experience as they traverse matter. The scattering media used in this study is water due to its close analog to human tissue. 
For the energy range of positrons used in PET, there are two main physical processes that determine the positron's path. The majority of the positron's deflection is due to elastic collisions with atomic nuclei. Bhabha scattering with orbital electrons also occurs (although not nearly as frequently) and can result in large angular deflections due to the equal masses of the positron and the electron. The majority of the energy lost is due to interactions with the atomic electrons, while only a small amount of energy will be lost to Bremsstrahlung radiation. The deflection of beta particles due to the Coulomb force with atomic nuclei and the continuous energy loss due to interactions with atomic electrons are described by Molière's theory and the Bethe-Bloch equation, respectively. EGS4 uses modifications of these theories to track the path of each positron and all secondary charged particles created in the shower process (The secondary particles are of no interest to this study and will not be discussed further).

The EGS4 simulation uses several separate routines to simulate the energy loss and scattering angle of the positron for each simulation step. For positrons traveling through a medium the relevant interactions for PET based applications include the continuous energy losses due to both Bremsstrahlung interactions and inelastic Coulomb scattering off of the atomic electrons and nuclei, elastic scattering with atomic nuclei of the medium, and Bhabha scattering. The change in energy that occurs over each simulation step is estimated by the energy lost due to both Bremsstrahlung radiation and the Coulomb forces experienced between the scattering particle and the electrons of the atoms (these interactions have a small scattering angle). A change in positron trajectory is calculated at the end of each simulation step using Molière's scattering theory. The change in positron's kinetic energy is 


\begin{tabular}{c|cc} 
Event Type & Number of Interactions & Percent of Total \\
\hline Molière + Continuous Energy Loss & 948,194 & 95.3 \\
Bhabha Scattering & 46,469 & 4.7 \\
Bremsstrahlung & 35 & $3.5 \times 10^{-3}$
\end{tabular}

Table 2.2: Relative physics interaction probabilities for 994,698 EGS4 interaction steps from 1000 simulated ${ }^{68} \mathrm{Ga}$ positron trajectories with initial kinetic energies randomly selected from the ${ }^{68} \mathrm{Ga}$ positron range distribution.

simulated using a the combination of the Bethe-Bloch equation and Bremsstrahlung losses. Table 2.2 tabulates the number of each type of interaction that occurs for positrons emitted from a ${ }^{68} \mathrm{Ga}$ point source in water. One thousand random events with initial kinetic energies sampled from the ${ }^{68} \mathrm{Ga}$ positron energy spectrum were used to generate this data. As can be seen from the table, the majority of the events undergo Molière scattering, and the positron kinetic energy is modified by the continuous energy loss approximation. Bhabha scattering events occur, but are very rare and will not significantly affect the PET data. As can be seen from the simulated data, Bremsstrahlung events are very rare.

\subsubsection{Molière's multiple scattering theory}

Molière's multiple scattering theory describes the probability that an incident beta particle will scatter into an angle $\theta$ due to the net Coulomb force of from the local atomic nuclei in a scattering material with atomic number $Z$ after traversing through a given thickness $t$. Molière scattering is considered to be elastic due to the large mass difference between the scattered beta and the atomic nuclei. Coulomb scattering off the nucleus is the dominant process responsible for the deflection of the positron's path through the body. The small angle deflection due to the interactions between 
the positron and the atomic electrons can thus be neglected when calculating the scattering angle due to the Coulomb force.

The following physical constants are used in the Molière's multiple scattering theory: $\alpha$ is the fine structure constant, $\lambda^{\prime}$ is the electron DeBroglie wavelength, and $a_{0}$ is the Bohr radius. Molière's scattering theory is described by the following set of equations [24]:

$$
f(\theta) \theta d \theta=\lambda d \lambda \int_{0}^{\infty} y d y J_{0}(\lambda y) \exp \left[\frac{1}{4} y^{2}\left(-b+\ln \left(\frac{1}{4} y^{2}\right)\right)\right]
$$

where $y$ is a integration variable, $\lambda=\theta / \chi_{c}$, and $J_{0}$ is the zeroth order Bessel function. The value $b$ is given by

$$
b=\ln \left[\frac{\chi_{c}^{2}}{1.167 \chi_{a}^{2}}\right]
$$

where $e^{b}$ is an estimate of the number of scattering interactions $\Omega_{0} \cdot \chi_{c}$ sets a limit on the minimum scattering angle that can occur and is given by

$$
\chi_{c}^{2}=\frac{4 \pi N t e^{4} Z(Z+1)}{(p v)^{2}}
$$

$\chi_{a}$ is the characteristic screening angle given by

$$
\chi_{a}^{2}=\chi_{0}^{2}\left(1.13+3.76 \alpha^{2}\right)
$$

Finally, $\chi_{0}$ is the critical angle given by

$$
\chi_{0}=\frac{\lambda^{\prime}}{0.885 a_{0} Z^{-1 / 3}}
$$

Scattering angles below $\chi_{0}$ deviate from the Rutherford scattering law due to nuclear effects [24].

Molière's scattering theory, as described by Bethe [25], is accurate only if the number of scattering interactions $\Omega_{0}$ per unit length $t$ is greater than 20 [24]. Molière's 
multiple scattering theory will begin to break down for positron's with energy less than $50 \mathrm{keV}$ (they will only travel $0.06 \mathrm{~mm}$, and $\Omega_{0} \approx 20$ ) [24]. As a consequence, this puts a lower limit on the size of ESTEPE in the EGS4 simulation. If the step size is too small, resulting in less than 20 collisions per step, Molière's theory will not be valid leading to an incorrect scattering angle. However, if the ESTEPE is made too large the magnetic field bending subroutine will no longer yield a good approximation

of the magnetic force (see section 2.5). For our simulation an ESTEPE of 1 percent of the particle's current total energy is recommended for accurately tracking positrons below $1500 \mathrm{keV}$ [21]. This step size is small enough to accurately model magnetic field influences (discussed in section 2.5) and still properly simulate multiple elastic scattering interactions.

\subsubsection{Bhabha Scattering}

Bhabha is the inelastic scattering of an electron and a positron. In our simulation, Bhabha scattering occurs when a positron transfers a portion of its kinetic energy to an electron much greater than the atomic ionization energy. In this situation the electron can be considered to be both free and at rest.

The physics used to determine the energy transfered to the electron and the angle of scattering of both particles is a two body scattering problem (see Fig. 2.4). A straight forward technique to evaluate the physics of this scattering interaction is to use the four-momentum of each particle, which is simply each particle's momentum in three dimensions plus its energy. The momentum four vectors for the initial and 


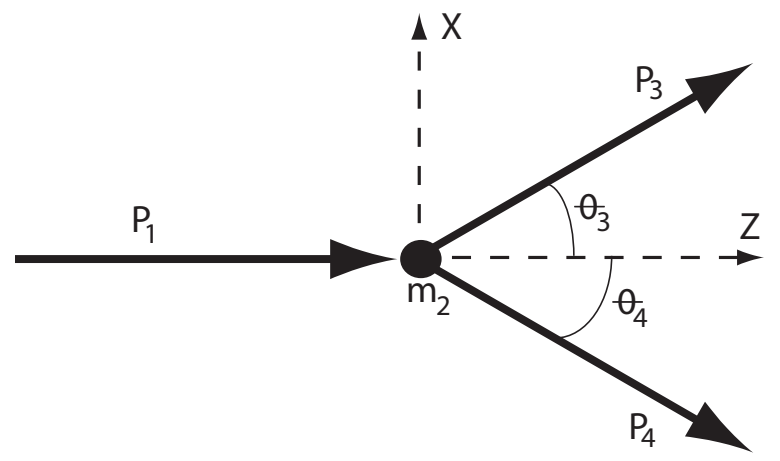

Figure 2.4: Generic two body scattering diagram.

final states are given by :

$$
\begin{array}{r}
P_{1}=\left(E_{1}, 0,0, p_{1}\right) \\
P_{2}=\left(m_{2}, 0,0,0\right) \\
P_{3}=\left(E_{3}, p_{3} \sin \theta_{3}, 0, p_{3} \cos \theta_{3}\right) \\
P_{4}=\left(E_{4},-p_{4} \sin \theta_{4}, 0, \cos \theta_{4}\right)
\end{array}
$$

where $\mathrm{c}=1$ in the following analysis, $E_{1}, E_{3}$, and $E_{4}$ are the total energy of each particle, $m_{2}$ is the rest mass energy of the electron and $\theta_{3}$ and $\theta_{4}$ are the scattering angles defined in Fig. 2.4.

The total four-vector momentum of the system must be conserved:

$$
P_{1}+P_{2}=P_{3}+P_{4}
$$

Solving for $P_{4}$ in equation 2.13 and squaring the result yields

$$
P_{4}^{2}=P_{1}^{2}+P_{2}^{2}+P_{3}^{2}-2\left(P_{1}+P_{2}\right) \cdot P_{3}+2 P_{1} \cdot P_{2}
$$

The scattering angle of the positron $\theta_{3}$ can be found by taking the dot products between $P_{1}$ and $P_{3}, P_{2}$ and $P_{3}, P_{1}$ and $P_{2}$ and substituting $P^{2}=m^{2}$ in equation 2.14 . 
The result for the positron scattering angle is given by

$$
\theta_{3}=\cos ^{-1}\left(\frac{m_{4}^{2}-m_{3}^{2}-m_{2}^{2}-m_{1}^{2}+2\left(E_{1}+m_{2}\right) E_{3}-2 E_{1} m_{2}}{2 p_{1} p_{3}}\right)
$$

where $p_{1}$ and $p_{3}$ are the magnitudes of the $P_{3}$ and $P_{4}$ four momenta, respectively.

The differential cross sectional data generated in PEGS4 and used in the EGS4 simulation for Bhabha scattering is given by [21]:

$$
\frac{d \Sigma_{\text {Bhabha }}\left(E_{0}\right)}{d E_{-}}=\frac{X_{0} n 2 \pi r_{0}^{2} m}{T_{0}^{2}}\left[\frac{1}{\mathcal{E}}\left(\frac{1}{\mathcal{E} \beta^{2}}-B_{1}\right)+B_{2}+\mathcal{E}\left(\mathcal{E} B_{4}-B_{3}\right)\right]
$$

where $E_{0}$ is the incident positron total energy $(\mathrm{MeV}), T_{0}$ is the incident positron kinetic energy $(\mathrm{MeV}), r_{0}$ is the classical electron radius in $\mathrm{cm}, X_{0}$ is the radiation length in $\mathrm{g} \mathrm{cm}^{-2}, \beta=v / c, E_{-}$is the secondary electron energy $(\mathrm{MeV}), \mathcal{E}=\left(E_{-}-\right.$ $m) / T_{0}, B_{1}=2-y^{2}, B_{2}=B_{4}+(1-2 y)^{2}, B_{4}=(1-2 y)^{3}, y=1 /(\gamma+1)$, and $\gamma=E_{0} / m$. Integrating equation 2.16 between two energy values $E_{1}$ and $E_{2}$

$$
\sigma_{E_{1}-E_{2}}=\int_{E_{1}}^{E_{2}} \frac{d \Sigma_{\text {Bhabha }}\left(E_{0}\right)}{d E_{-}} d E_{-}
$$

will give the cross section for an electron to be scattered with an energy between $E_{1}$ and $E_{2}$. Once the energy of the scattering particles are selected from the Bhabha cross sectional data, the scattering angles for the positron and electron are determined using equation 2.15. A random angle $\phi$ from 0 to $2 \pi$ is randomly chosen as the azimuthal angle about the z-axis.

\subsubsection{Inelastic energy losses due to soft collisions}

In the EGS4 simulation the continuous energy loss per unit of distance due to both inelastic Coulomb scattering with the atomic electrons and in the creation of a Bremsstrahlung photons is given by

$$
-\left(\frac{d E_{ \pm}}{d x}\right)_{\text {TotalCont }}=-\left(\frac{d E_{ \pm}}{d x}\right)_{\text {SoftBrems }}-\left(\frac{d E_{ \pm}}{d x}\right)_{\text {Sub-cutoff-Atomic-Elect }}
$$




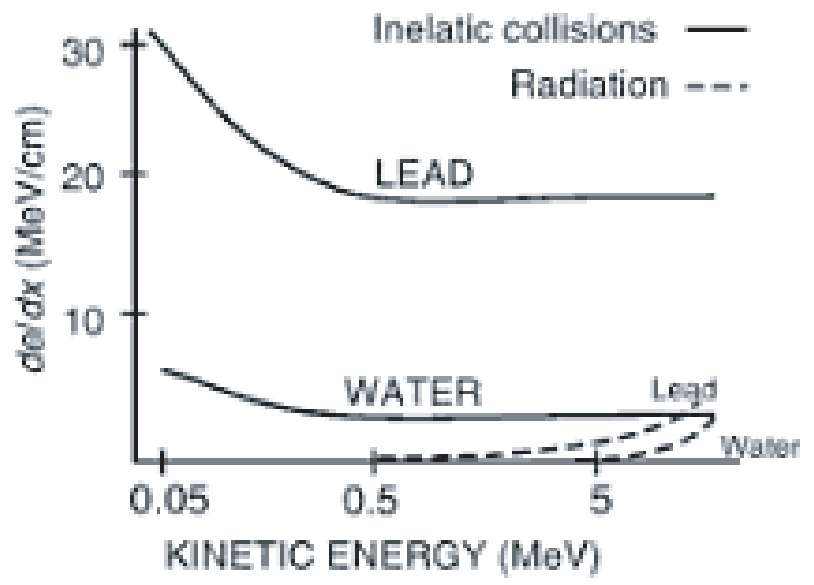

Figure 2.5: Energy loss per unit length $d E / d x$ for beta particles as a function of kinetic energy due to inelastic collisions and Bremsstrahlung radiation in water and lead. Image taken from [1].

The energy transfered to the orbital electrons in this approximation is insufficient to ionize the atom and no secondary electrons are created in the energy transfer.

Figure 2.5 is a plot comparing the change in energy per unit distance as a function of the kinetic energy of a charged particle in lead and water for energy lost by both Bremsstrahlung and inelastic atomic electron excitations. As can be seen from the plot, the continuous energy loss due to the Coulomb interactions between the incident charged particle and the atomic electrons is the dominant contribution to the incident particle's energy loss. It is not until the incident particle's kinetic energy is above $5 \mathrm{MeV}$ that there is a noticeable rise in the probability of Bremsstrahlung photon production in water. The maximum positron energy used in PET imaging is on the order of $2.5 \mathrm{MeV}$; consequently, energy loss due to Bremsstrahlung radiation is not a 
dominant physical mechanism for the energy loss of positrons through the patient's body (as was previously shown using the EGS4 simulation, Table 2.2).

The cross-section data used in EGS4 for Bremsstaulung scattering is taken from a review article by Koch and Motz [26]. The following equation illustrates the dominant quantities that influence Bremsstraulung interactions without the complications that would be introduced by discussing the full scattering formula.

$$
\sigma_{\text {Brems }} \sim Z^{2}\left(\frac{e^{2}}{m_{e} c^{2}}\right)^{2} f(E)
$$

where $f(E)$ is a strongly increasing function of the incident beta energy. The energy loss per unit length lost by the incident beta traversing matter is given by:

$$
\left(-\frac{d E}{d x}\right)_{\text {Brems }} \sim N E \sigma_{\text {Brems }}
$$

where $\mathrm{N}$ is the atomic density of the material.

The energy lost per unit distance for charged particles inelastically scattering off of the electrons in a media is given by the Bethe-Bloch equation [6]:

$$
\frac{d E}{d x}=4 \pi r_{0}^{2} \frac{m c^{2}}{\beta^{2}} N Z(A+B)
$$

where

$$
A=\ln \frac{\beta \gamma \sqrt{\gamma-1} m c^{2}}{I}
$$

and

$$
B=\frac{1}{2 \gamma^{2}}\left[\frac{(\gamma-1)^{3}}{8}+1-\left(2 \gamma^{2}+2 \gamma-1\right) \ln 2\right]
$$

where $r_{o}$ is the classical electron radius $=2.82 \times 10^{-13} \mathrm{~cm}, \gamma=(1-\beta)^{-1 / 2}, \beta=v / c$, $\mathrm{N}$ is atomic density of the atoms in the scattering medium, $\mathrm{Z}$ is the atomic number, and $\mathrm{I}$ is the mean excitation potential of the medium measured in $\mathrm{eV}$. 
The original Bethe-Block equation is valid in the limit that the incident particle interacts only with a single atom at a time, which is a good approximation for gases. However, for solids and liquids the atoms are more closely packed and the electrical polarization of the material must be taken into account. For dense scattering media the dielectric constant of the material will weaken the effective electric field of the atom at a distance. This decrease in the electric field will result in less energy transfered from the incident charged particle to the atomic electrons. This effect is only relevant for charged particle velocities approaching the speed of the light. An extensive study of this effect for electrons traveling through various materials including water and silicon can be found in [27] and [28] respectively.

The EGS4 simulation uses a modified version of the Bethe-Block equation that takes into account a density correction leading to a restricted stopping power for the continuous energy loss of electrons and positrons scattering through a medium [21], [29], [30]. The modified Bethe-Bloch equation is given by:

$$
\left(-X_{0} \frac{d E_{ \pm}}{d x}\right)_{\text {sub-cut-atom-elec }}=\frac{2 \pi X_{0} n r_{0}^{2} m}{\beta^{2}}\left[\ln \frac{2(r+2)}{(I / m)}+F^{ \pm}(r, \Delta)-\delta\right]
$$

with

$$
\begin{aligned}
& F^{-}(r, \Delta)=-1-\beta^{2}+\ln [(r-\Delta) \Delta]+\frac{r}{r-\Delta}+\left[\frac{\Delta^{2}}{2}+(2 r+1) \ln \left(1-\frac{\Delta}{r}\right)\right] / \gamma^{2}(2.25) \\
& F^{+}(r, \Delta)=\ln (r \Delta)-\frac{\beta^{2}}{r}\left[r+2 \Delta-\frac{3 \Delta^{2} y}{2}-\left(\Delta-\frac{\Delta^{3}}{3}\right) y^{2}-\left(\frac{\Delta^{2}}{2}-\frac{r \Delta^{3}}{3}+\frac{\Delta^{4}}{4}\right) y^{3}\right]
\end{aligned}
$$

where $E_{0}$ is the incident beta energy $(\mathrm{MeV}), X_{0}$ is the radiation length $(\mathrm{cm}), \delta=$ $E_{0} / m$ is the relativistic factor, $\eta=\sqrt{\gamma^{2}-1}=\beta \gamma=p_{0} c / m, \beta=\sqrt{1-\gamma^{-2}}=v / c$, $r=\gamma-1, y=(\gamma+1)^{-1}, T_{E}^{\prime}$ is the electron cut off energy $(\mathrm{MeV}), T_{\text {max }}^{\prime}$ is the maximum energy transfer ( $\mathrm{r}$ for positrons, $\mathrm{r} / 2$ for electrons), $\Delta=\min \left(T_{E}^{\prime}, T_{\max }^{\prime}\right.$ ) is 


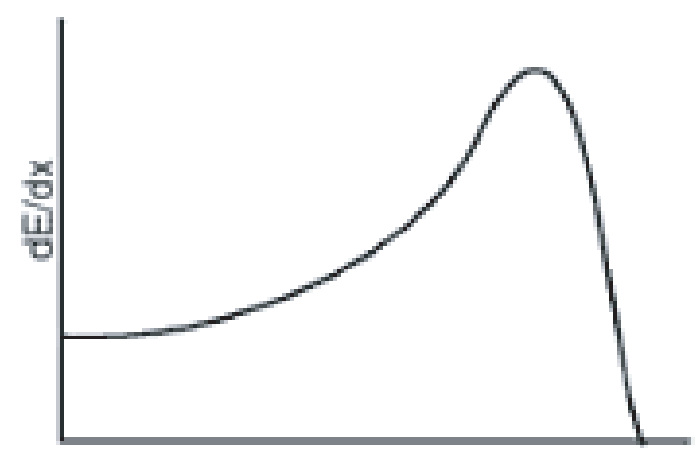

PENETRATION DEPTH

Figure 2.6: Example shape of $\frac{d E}{d x}$ versus penetration depth due to the interaction of a beta particle scattering through matter as it losses energy via inelastic collisions with atomic electrons. Image taken from [1].

the restricted maximum energy transfer,$I$ is the average mean ionization energy, and $\delta$ is density effect correction. A list of I values for elements, mixtures, and compounds can be found in [21]. A detailed explanation and derivation of the density correction factor $\delta$ for various materials is given in [27] [28]. Figure 2.6 is a plot of the typical change in energy over distance $\frac{d E}{d x}$ versus penetration depth for a beta particle due to inelastic electron scattering described by the Bethe-Bloch equation. As can be seen in the plot the particle deposits the majority of its energy at the end of its trajectory. The peak in this image is known as the Bragg peak.

The positron follows a tortuous path as it scatters through the media as a result of the physical interactions discussed in this section. Figure 2.7 is an example of ten positron paths projected onto a single plane for $1900 \mathrm{keV}$ positrons emitted in ten random directions in water. Each positron trajectory ends with an annihilation event which is discussed in section 2.6 . 


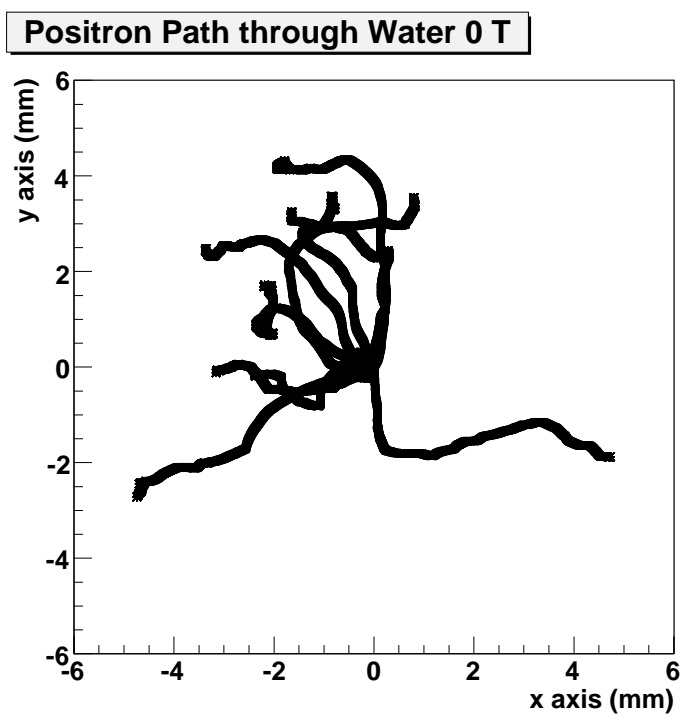

Figure 2.7: Simulated positron trajectories projected onto a plane for ten $1900 \mathrm{keV}$ positrons emitted at various angles in water.

\subsection{The effect of magnetic fields on charged particles}

Charged particles moving through a magnetic field will experience a Lorentz force transverse to both the velocity and magnetic field vectors. The equation for the magnetic force on a charged particle moving through a magnetic field is given by:

$$
\mathbf{F}=q(\mathbf{v} \times \mathbf{B})
$$

where $\mathrm{q}$ is the charge of the particle, $\mathbf{v}$ is the velocity, and $\mathbf{B}$ is the magnetic field. The magnitude of the magnetic force is given by:

$$
F=q v B \sin \theta
$$

where $\theta$ is the angle between the velocity and magnetic field vectors. As can be seen from equation 2.27, the component of the velocity aligned with the magnetic 
field vector will not experience a force; however, the component of the particle's velocity transverse to the magnetic field will experience a force perpendicular to the instantaneous direction of motion. As a result, a charged particle traveling through a magnetic field in vacuum will trace out a helix. For the EGS4 simulations, the positron scatters through water, and thus, its path will not trace out a simple helix. Instead, the positron will follow a tortuous path as discussed in the previous sections. The advantage of using a magnetic field is that the magnetic force will cause the positron to curve between physics interactions, resulting in a positron-electron annihilation event that will be (on average) closer to the positron emission point.

The magnetic force that acts the positron is implemented in the EGS4 simulation by modifying the direction of the positron's trajectory after each simulation step using the UCBEND subroutine [21]. The change in the particle's direction in UCBEND is calculated by using the approximation that both the distance between each simulated step $\Delta l$ and the change in the particle's energy over that step are small. With this approximation the direction of the velocity unit vector at the end of each step can be expressed as [21]:

$$
\hat{v}=\hat{B}\left(\hat{v_{0}} \cdot \hat{B}\right)+\left[\hat{v}_{0}-\hat{B}\left(\hat{v_{0}} \cdot \hat{B}\right)\right] \cos \alpha-\left(\hat{v}_{0} \times \hat{B}\right) \sin \alpha
$$

where $\alpha=\Delta l / R, R=p / q B$ is the radius of curvature of the particle, $p$ is the particle

momentum, $\hat{B}$ is the magnetic field unit vector, $\hat{v}_{0}$ is the particle's initial velocity unit vector, and $\hat{v}$ is the particle's velocity unit vector after a step $\Delta l$.

Figure 2.8 is an example of ten $1900 \mathrm{keV}$ positron trajectories in water projected onto a plane transverse to a $7 \mathrm{~T}$ magnetic field. It is evident that the $7 \mathrm{~T}$ positron paths terminate closer to the origin (the positron's emission point) in comparing the $0 \mathrm{~T}$ trajectories in water (Fig. 2.7. Although the total path length traveled by the 


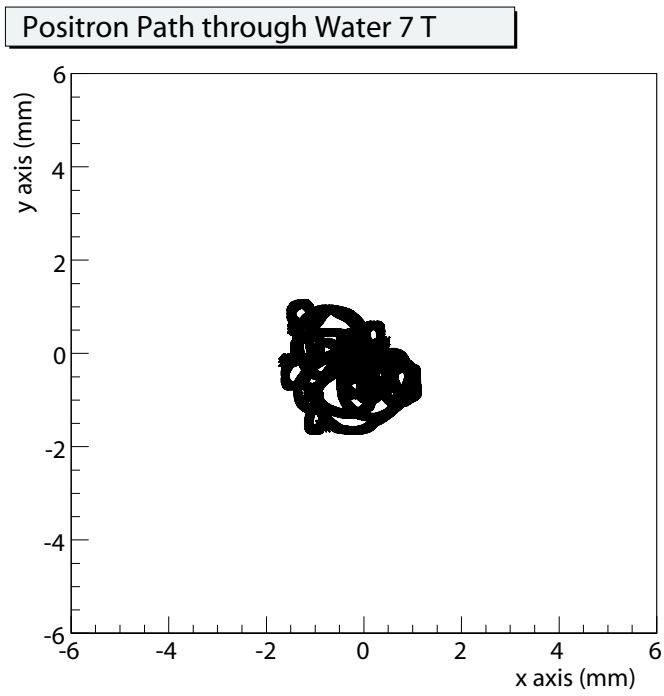

Figure 2.8: Simulated positron trajectories projected onto a plane of ten $1900 \mathrm{keV}$ positrons emitted at various angles in water. A $7 \mathrm{~T}$ magnetic field is directed transverse to the plane.

positron is unchanged in the presence of a magnetic field, the displacement (range) of the average final annihilation position will be reduced at $7 \mathrm{~T}$ compared to $0 \mathrm{~T}$. By reducing the range of the positron with a strong magnetic field higher resolution images can be obtained in the plane transverse to the magnetic field; however, no improvement in resolution will be observed in the direction of the magnetic field.

\subsection{Positron-electron annihilation and Doppler Broadening}

An annihilation event occurs when a positron and electron come into contact and are replace by two nearly collinear $511 \mathrm{keV}$ gamma rays. The two gamma rays produced in the annihilation event will be emitted perfectly collinear only if the total momentum of the positron-electron system is zero. However, the positron usually annihilates with an electron that is bound to an atom in the patient's body. This 


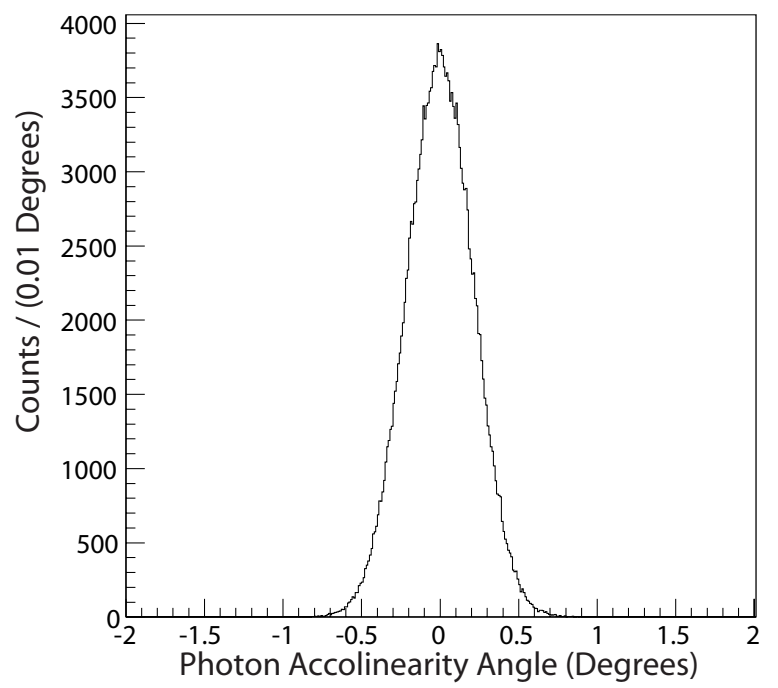

Figure 2.9: Histogram of random photon acollinearity angles. The distribution is Gaussian with a $0.5 \mathrm{~mm}$ FWHM. The bin width is 0.01 degrees.

bound electron is rotating about the nucleus and has a non-zero momentum. In this situation, the momentum of the positron-electron system is non-zero. In order for momentum to be conserved the photons are emitted at an angle that deviates slightly from the ideal 180 degree emission angle. This effect is known as Doppler broadening and is a function of the medium in which the annihilation event occurs.

The annihilation photon acollinearity angle in water has been measured experimentally by Shibuya, et al. for $511 \mathrm{keV}$ photons [11]. In this study it was found that the acollinearity angle is Gaussian with a width of 0.5 degrees FWHM. Figure 2.9 is a plot of randomly generated photon acollinearity angles used in this simulation. The photon acollinearity angles are used as inputs for the EGS4 photon tracking simulation. 


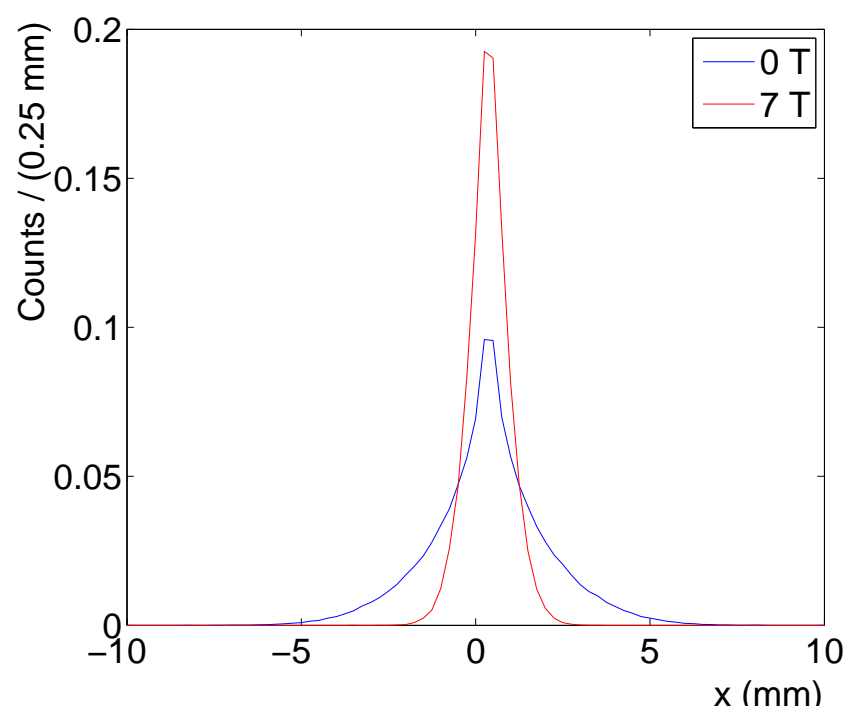

Figure 2.10: EGS4 simulation results of the x-projections of the ${ }^{68} \mathrm{Ga}$ positron annihilation distribution in water at $0 \mathrm{~T}$ and with a $7 \mathrm{~T}$ magnetic field applied in the z-direction. The area under the plots has been normalized to one.

\subsection{Results of Positron Range Simulations}

Several groups have performed Monte Carlo simulations to study the range of the positron through water for various radioisotopes commonly used in PET [24] [31] [32]. Our simulation results are consistent with the referenced studies for common radioisotope point sources embedded in water at $0 \mathrm{~T}$. Due to the cusp like distribution and the associated tails of the projected annihilation points, the full-width-at-tenthmaximum (FWTM), in addition to the full-width-at-half-maximum (FWHM), is an important quantity to consider (see Fig. 2.10). This is especially true when dealing with higher energy positron emitters. The FWHM and FWTM values are extracted from the projection of the three dimensional positron annihilation distribution onto a single axis. 


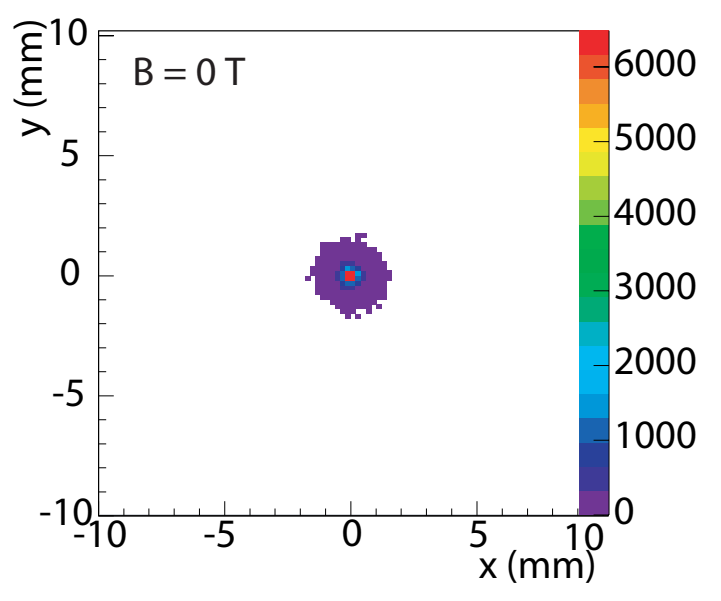

Figure 2.11: Two dimensional density histogram of the EGS4 simulation results of ${ }^{22} \mathrm{Na}$ positron annihilation positions in water at $0 \mathrm{~T}$.

The results of our EGS4 simulation for the low energy positron emitter ${ }^{22} \mathrm{Na}(535$ keV maximum positron energy) are shown in Figs. 2.11 and 2.13. Figure 2.11 is the positron annihilation distribution projected onto the xy-plane at 0 T. Figure 2.13 are the ${ }^{22} \mathrm{Na}$ positron range distributions projected onto two orthogonal planes in the presence of a $7 \mathrm{~T}$ magnetic field directed along the z-axis. The left image in Figure 2.13 is the positron annihilation distribution projected onto the xy-plane (transverse to the magnetic field) while the image on the right is a projection onto the xz-plane. Table 2.4 summarizes the results of this simulation by listing the FWHM and FWTM values of the $0 \mathrm{~T}$ and $7 \mathrm{~T}^{22} \mathrm{Na}$ positron annihilation distributions in water projected onto the x-axis (these projections are shown in Figs. 2.12 and 2.14 for $0 \mathrm{~T}$ and $7 \mathrm{~T}$, respectively). These simulation results suggest that the effect of the positron range on the image resolution of PET scanners for low energy positron emitters may improve slightly in the presence of strong magnetic fields in normal, water equivalent tissue. 

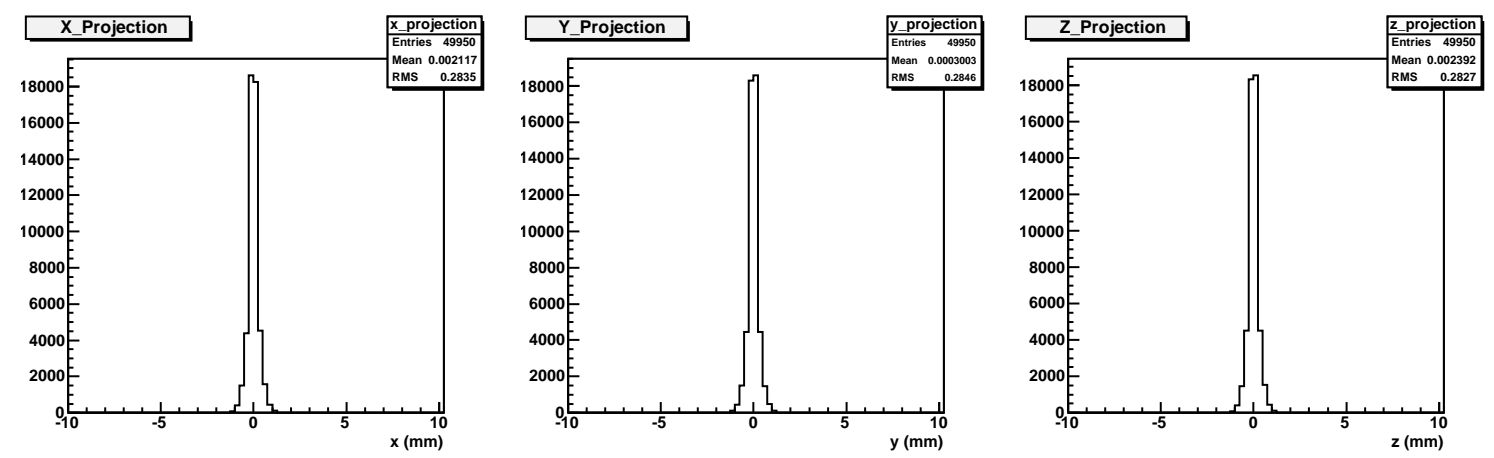

Figure 2.12: Projections of the ${ }^{22} \mathrm{Na} 0 \mathrm{~T}$ positron-electron annihilation distribution in water along the $\mathrm{x}, \mathrm{y}$, and $\mathrm{z}$ axes, respectively.

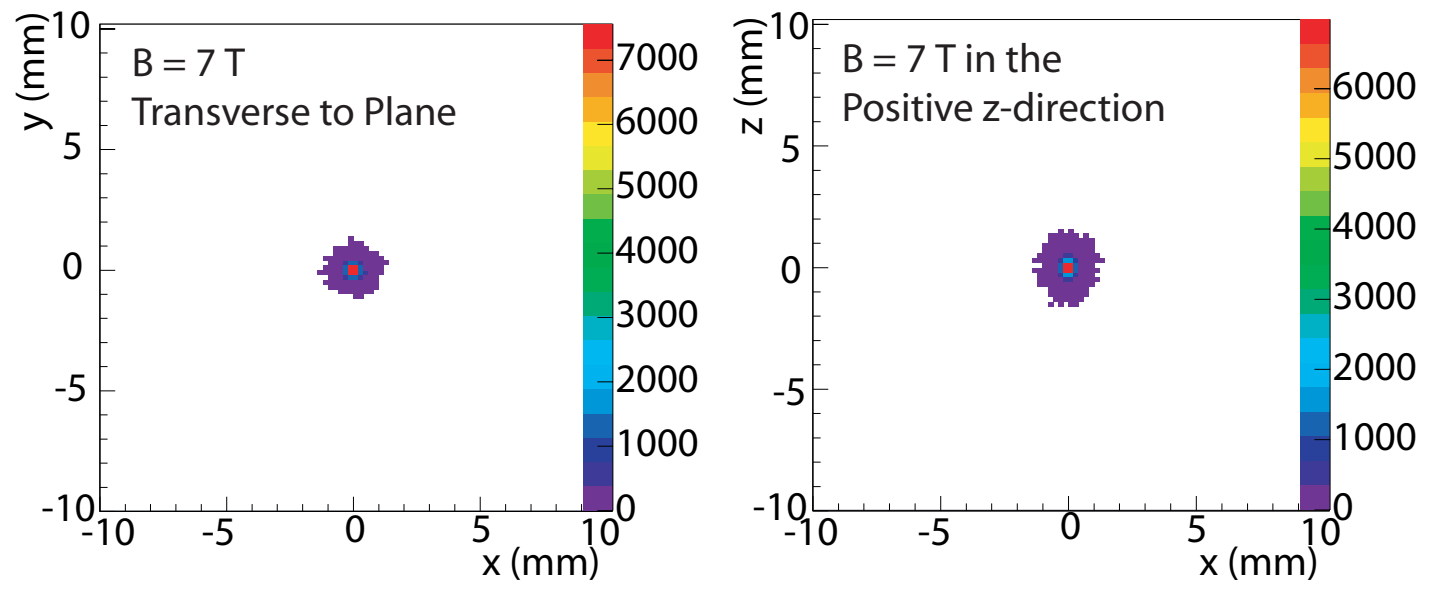

Figure 2.13: Two dimensional density histograms of the EGS4 simulation results of ${ }^{22} \mathrm{Na}$ positron annihilation positions in water at $7 \mathrm{~T}$ magnetic field applied transverse to the image plane (left) and a $7 \mathrm{~T}$ magnetic field in the positive z-direction (right) 

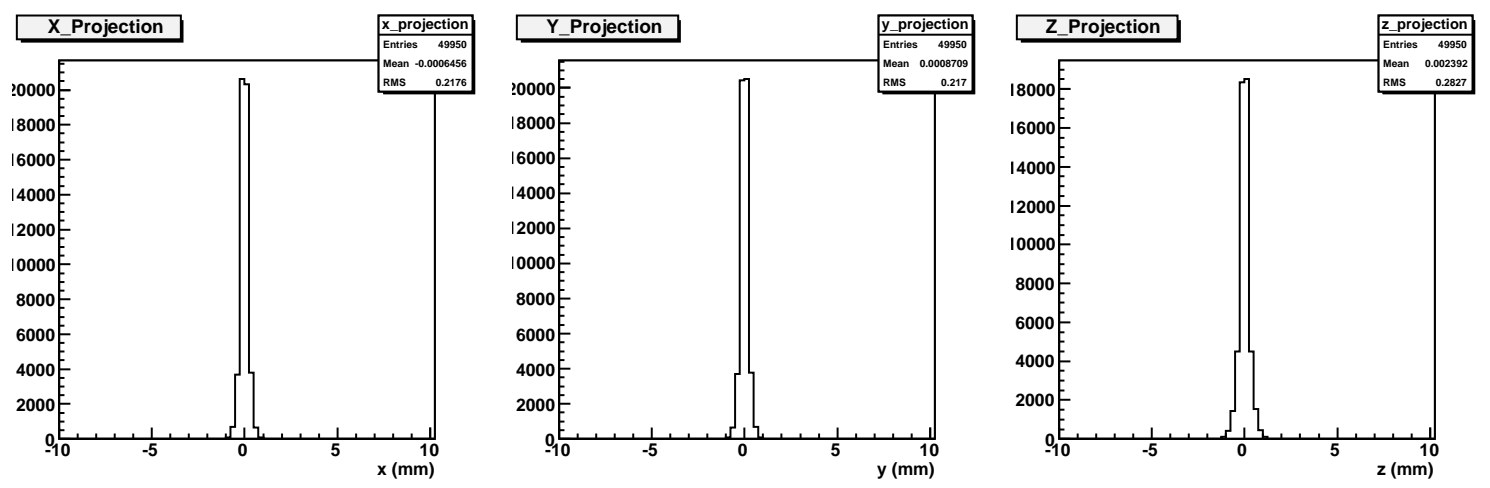

Figure 2.14: Projections of the ${ }^{22} \mathrm{Na} 7 \mathrm{~T}$ positron-electron annihilation distribution in water along the $\mathrm{x}, \mathrm{y}$, and $\mathrm{z}$ axes, respectively.

The effect of the magnetic field on the positron annihilation positions is especially impressive for higher energy radioisotopes such as ${ }^{68} \mathrm{Ga}(1899 \mathrm{keV}$ maximum positron energy). The scatter plots shown in Figs. 2.15 and 2.17 are the projections of the ${ }^{68} \mathrm{Ga}$ distribution of the positron annihilation points onto a plane for both $0 \mathrm{~T}$ and 7 T. Figure 2.15 is a scatter plot of the positrons' annihilation points in the xyplane in the absence of a magnetic field. For the images in Fig. 2.17 a uniform $7 \mathrm{~T}$ magnetic field has been applied in the positive z-direction. The image on the left is a projection of the positron annihilation positions onto the plane transverse to the $7 \mathrm{~T}$ magnetic field (xy-plane), while the image on the right is a projection onto the xzplane. As expected, the variations in the annihilation points are reduced in the plane transverse to the applied magnetic field. Likewise, the projection into the xz-plane shows a reduction in the positron range in the $\mathrm{x}$-direction, but no improvement in the z-direction (the direction of the applied magnetic field). Figure 2.10 compares the $0 \mathrm{~T}$ and $7 \mathrm{~T}$ simulation results of the projection of the annihilation points onto the $\mathrm{x}$-axis for a ${ }^{68} \mathrm{Ga}$ point source embedded in water. Table 2.4 summarizes the results 
of this simulation by listing the FWHM and FWTM values of the $0 \mathrm{~T}$ and $7 \mathrm{~T}^{68} \mathrm{Ga}$ positron annihilation distributions in water projected onto the $\mathrm{x}$-axis (the projections are shown in Figs. 2.16 and 2.18 for $0 \mathrm{~T}$ and $7 \mathrm{~T}$, respectively). It is evident that with the application of a strong magnetic field the tails of the positron range can be greatly reduced, thus allowing for the potential of higher resolution images.

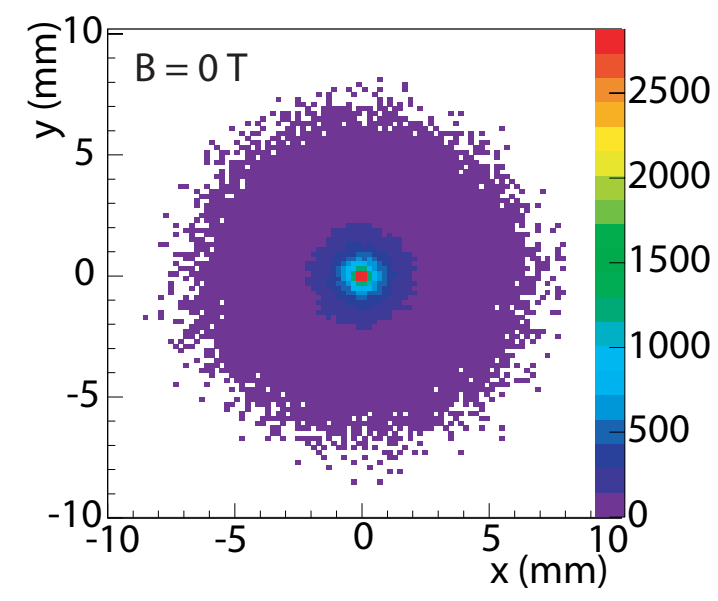

Figure 2.15: EGS4 simulation results of ${ }^{68} \mathrm{Ga}$ positron annihilation positions in water at $0 \mathrm{~T}$.

In order to extract the FWHM and FWTM values of the positron range distributions, the following procedure was followed. Since the positron range distribution is symmetric about the $\mathrm{x}$-axis for both $0 \mathrm{~T}$ and $7 \mathrm{~T}$ magnetic fields directed in the z-direction, the magnitude of the x-component of the positron range for each annihilation event is plotted. Each plot is normalized, such that the maximum value of the peak is equal to one. The $0 \mathrm{~T}$ distributions are then fit to the following function [24]:

$$
P(x)=C \exp \left(-k_{1} x\right)+(1-C) \exp \left(-k_{2} x\right)
$$



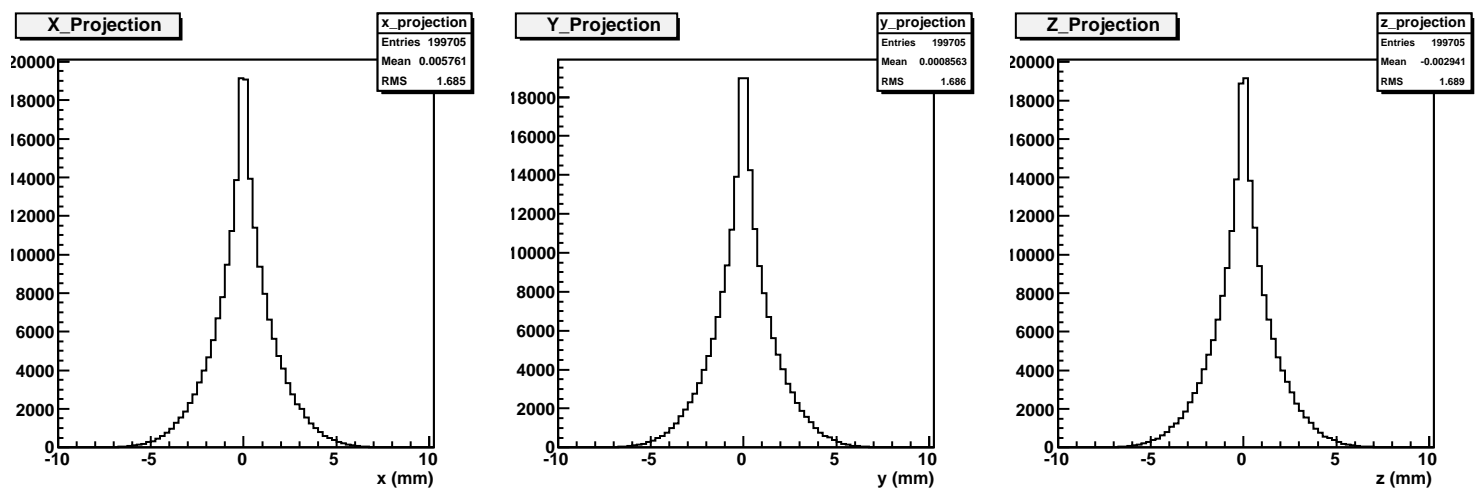

Figure 2.16: Projections of the ${ }^{68} \mathrm{Ga} 0 \mathrm{~T}$ positron-electron annihilation distribution in water along the $\mathrm{x}, \mathrm{y}$, and $\mathrm{z}$ axes, respectively.
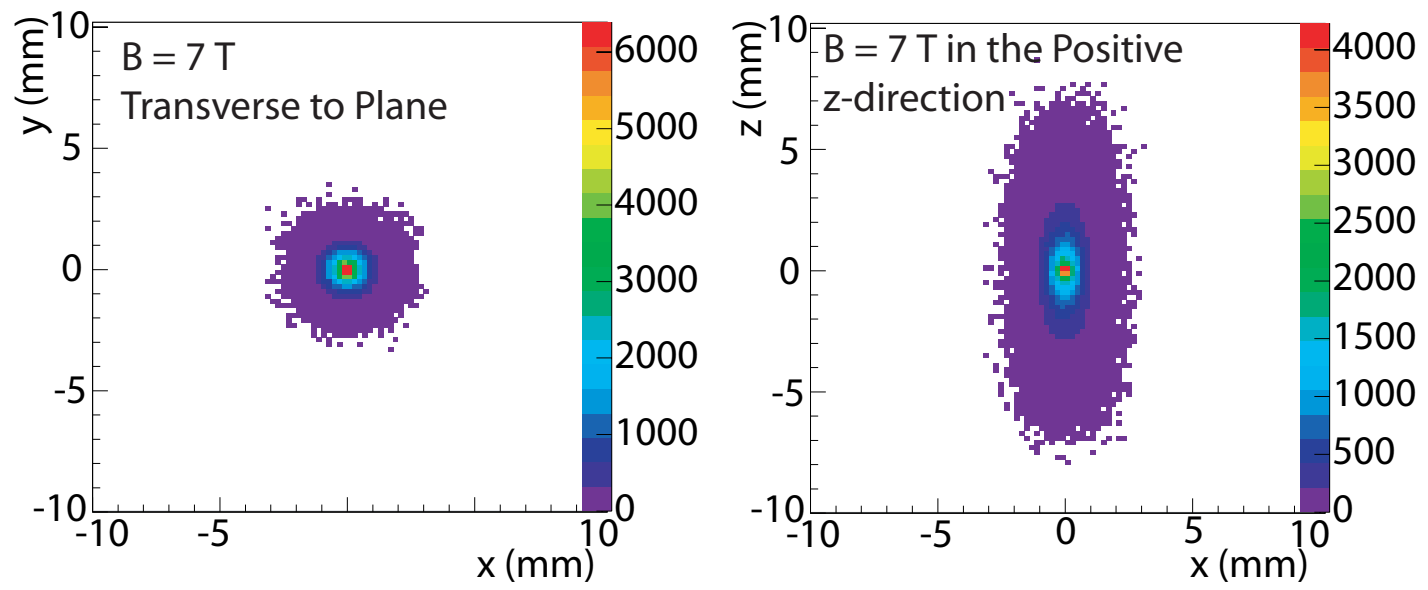

Figure 2.17: EGS4 simulation results of ${ }^{68} \mathrm{Ga}$ positron annihilation positions in water at $7 \mathrm{~T}$ magnetic field (left) applied transverse to the image plane and (right) $7 \mathrm{~T}$ magnetic field in the positive z-direction. 

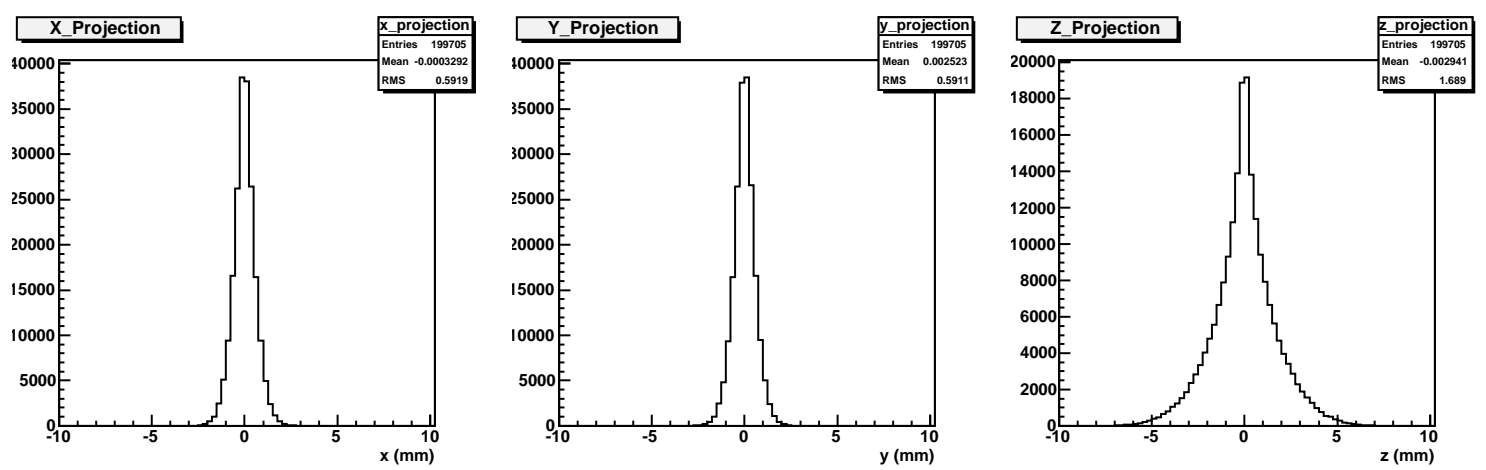

Figure 2.18: Projections of the ${ }^{68} \mathrm{Ga} 7 \mathrm{~T}$ positron-electron annihilation distribution in water along the $\mathrm{x}, \mathrm{y}$, and $\mathrm{z}$ axes, respectively.

\begin{tabular}{c|ccc} 
source & $\mathrm{C}$ & $k_{1}$ & $k_{2}$ \\
\hline${ }^{22} \mathrm{Na}$ & $0.608 \pm 0.006$ & $0.196 \pm 0.004$ & $0.0235 \pm 3 \times 10^{-4}$ \\
${ }^{68} \mathrm{Ga}$ & $0.282 \pm 0.005$ & $0.047 \pm 0.002$ & $3.62 \times 10^{-3} \pm 2.4 \times 10^{-5}$
\end{tabular}

Table 2.3: Fit parameters for $0 \mathrm{~T}$ positron range fits

where $C, k_{1}$, and $k_{2}$ are independent fit parameters and $x$ is the range in bins. Figures 2.19 and 2.21 are the $0 \mathrm{~T}$ fits to the $\mathrm{x}$-projections using equation 2.30. Table 2.3 contains the values of the fit parameters. Table 2.5 is a comparison between our 0 T EGS4 simulation results and the positron range simulation results of Levin and Hoffman, Sanchez et al., and Champion and Le Loirec [24] [31] [32]. Figures 2.20 and 2.22 are the magnitude of the annihilation positions projected onto the $\mathrm{x}$-axis for the $7 \mathrm{~T}$ magnetic field simulations for ${ }^{22} \mathrm{Na}$ and ${ }^{68} \mathrm{Ga}$, respectively. Both plots are fit to a standard double Gaussian function. The FWHM and FWTM were then extracted from the fits by finding the width at both half and tenth maximum and multiplying the result by two. The values are given in Table 2.4 


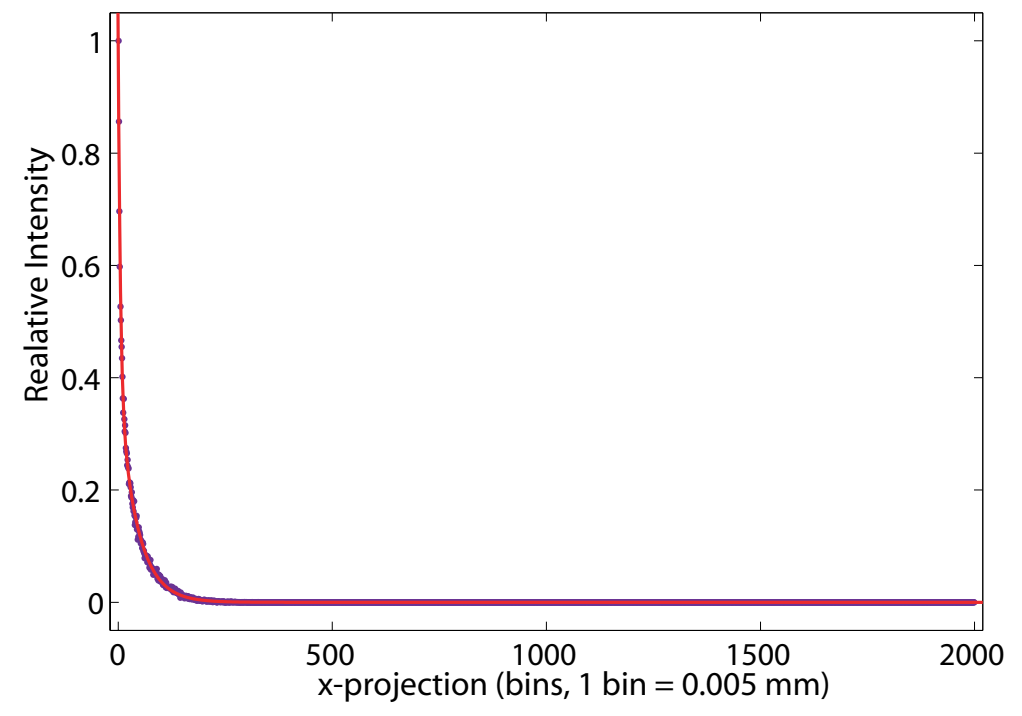

Figure 2.19: Fit of ${ }^{22} \mathrm{Na}$ EGS4 positron range annihilation x-projection of a point source at $0 \mathrm{~T}$ in water using the double exponential fit function 2.30. The FWHM and FWTM are $0.06 \mathrm{~mm}$ and $0.60 \mathrm{~mm}$, respectively. Each bin in the plot corresponds to $0.005 \mathrm{~mm}$.

\begin{tabular}{c|c|c|c|c} 
& ${ }^{22} \mathrm{Na}(0 \mathrm{~T})$ & ${ }^{22} \mathrm{Na}(7 \mathrm{~T})$ & ${ }^{68} \mathrm{Ga}(0 \mathrm{~T})$ & ${ }^{68} \mathrm{Ga}(7 \mathrm{~T})$ \\
\hline FWHM $(\mathrm{mm})$ & $0.082 \pm 0.002$ & $0.070 \pm 0.004$ & $1.01 \pm 0.06$ & $0.84 \pm 0.14$ \\
FWTM (mm) & $0.96 \pm 0.01$ & $0.54 \pm 0.02$ & $5.45 \pm 0.12$ & $2.28 \pm 0.14$ \\
rms (mm) & $0.25 \pm 2 \times 10^{-4}$ & $0.22 \pm 2 \times 10^{-4}$ & $1.15 \pm 1 \times 10^{-3}$ & $0.38 \pm 3 \times 10^{-4}$
\end{tabular}

Table 2.4: Simulation results of the positron annihilation distribution projected onto the $\mathrm{x}$-axis for both a ${ }^{22} \mathrm{Na}$ and a ${ }^{68} \mathrm{Ga}$ point source for magnetic field strengths of $0 \mathrm{~T}$ and $7 \mathrm{~T}$ in water. The magnetic field is oriented in the positive $\mathrm{z}$ direction for the $7 \mathrm{~T}$ simulation results. The rms values are calculated using the magnitude of the positron range distribution. 


\begin{tabular}{llll}
\hline Isotope & Simultion & FWHM $(\mathrm{mm})$ & FWTM (mm) \\
\hline${ }^{18} \mathrm{~F}$ & EGS4 & $0.082 \pm 0.002$ & $0.96 \pm 0.01$ \\
& Levin and Hoffman & 0.10 & 1.03 \\
& Sanchez, Andreo, and Larsson & 0.19 & 0.91 \\
& Champion and Le Loirec & 0.077 & 1.005 \\
${ }^{11} \mathrm{C}$ & EGS4 & $0.11 \pm 0.01$ & $1.40 \pm 0.02$ \\
& Levin and Hoffman & 0.188 & 1.860 \\
& Sanchez, Andreo, and Larsson & 0.28 & 1.70 \\
& Champion and Le Loirec & 0.088 & 1.855 \\
${ }^{13} \mathrm{~N}$ & EGS4 & $0.21 \pm 0.01$ & $1.85 \pm 0.04$ \\
& Levin and Hoffman & 0.282 & 2.530 \\
& Sanchez, Andreo, and Larsson & 0.33 & 2.12 \\
& Champion and Le Loirec & 0.152 & 2.599 \\
${ }^{15} \mathrm{O}$ & EGS4 & $0.28 \pm 0.03$ & $3.70 \pm 0.11$ \\
& Levin and Hoffman & 0.501 & 4.140 \\
& Sanchez, Andreo, and Larsson & 0.41 & 3.10 \\
& Champion and Le Loirec & 0.2341 & 5.157 \\
${ }^{68} \mathrm{Ga}$ & EGS4 & $1.01 \pm 0.06$ & $5.45 \pm 0.12$ \\
& Levin and Hoffman & - & - \\
& Sanchez, Andreo, and Larsson & 0.49 & 3.70 \\
& Champion and Le Loirec & 1.039 & 7.943 \\
\hline
\end{tabular}

Table 2.5: X-projection of the positron range for various positron emitters from four separate simulations. 

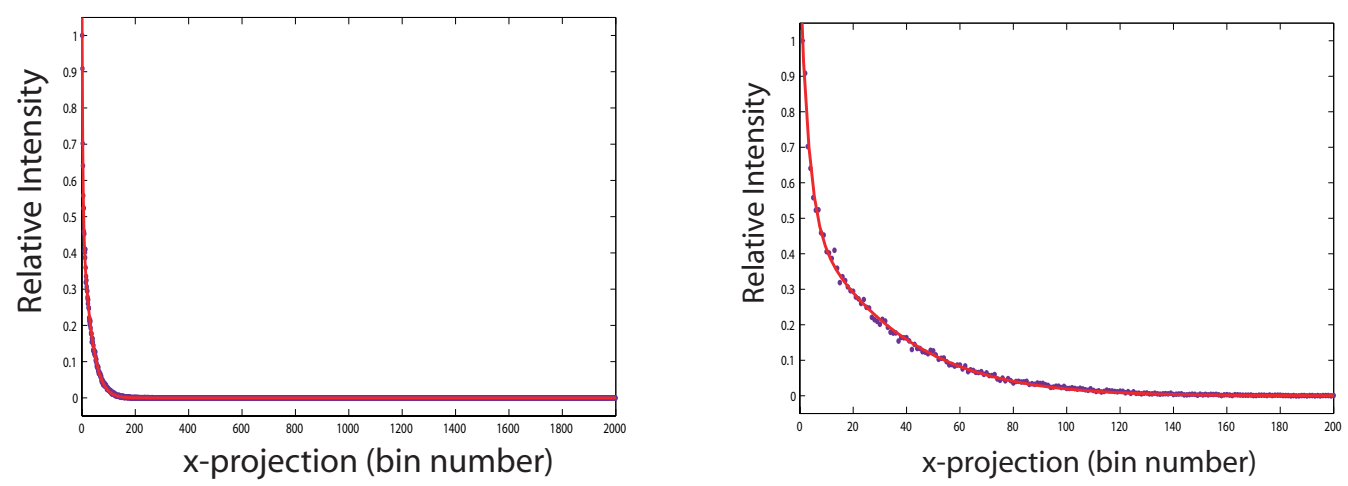

Figure 2.20: Fit of ${ }^{22} \mathrm{Na}$ EGS4 positron range annihilation x-projection of a point source at $7 \mathrm{~T}$ in water fit to a double Gaussian function. The FWHM and FWTM are $0.03 \mathrm{~mm}$ and $0.27 \mathrm{~mm}$, respectively. Each bin in the plot corresponds to 0.005 $\mathrm{mm}$.

The reduction of the positron range in strong magnetic fields is especially impressive in lung tissue. The following density plots are rough estimates of the positron annihilation positions in lung tissue. The PEGS4 cross-sections used in this study were calculated by scaling the density of water by one third. Figures 2.23 and 2.24 are images of ${ }^{22} \mathrm{Na}$ and ${ }^{68} \mathrm{Ga}$ point sources in "lung" tissue at $0 \mathrm{~T}$ (left) and with a 7 T magnetic field directed transverse to the plane (right). Table 2.6 lists the FWHM and FWTM of the x-projections of the distributions. It is important to note that these plots only use a very rough estimate for lung tissue, and as a result are not meant as a rigorous study. These plots are included to illustrate that the positron range correction in lung is likely to be greatly improved in the presence of strong magnetic field. For an in depth study of the positron range in lung, soft, fat, and bone tissues consult the simulation study by Sanchez, et. al. [31]. 


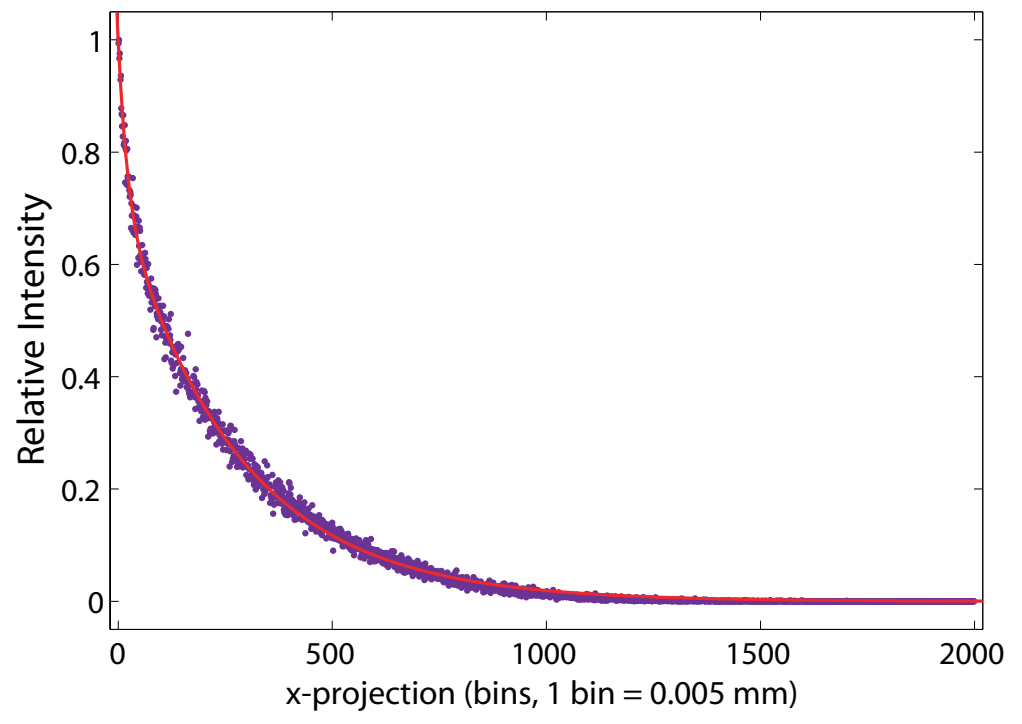

Figure 2.21: Fit of ${ }^{68} \mathrm{Ga}$ EGS4 positron range annihilation x-projection of a point source at $0 \mathrm{~T}$ in water using the double exponential fit function 2.30. The FWHM and FWTM are $1.02 \mathrm{~mm}$ and $5.45 \mathrm{~mm}$, respectively. Each bin in the plot corresponds to $0.005 \mathrm{~mm}$.

\begin{tabular}{c|c|c|c|c} 
& ${ }^{22} N a(0 \mathrm{~T})$ & ${ }^{22} \mathrm{Na}(7 \mathrm{~T})$ & ${ }^{68} \mathrm{Ga}(0 \mathrm{~T})$ & ${ }^{68} \mathrm{Ga}(7 \mathrm{~T})$ \\
\hline FWHM (mm) & $0.18 \pm 0.005$ & $0.30 \pm 0.006$ & $3.60 \pm 0.07$ & $1.10 \pm 0.02$ \\
FWTM (mm) & $1.80 \pm 0.04$ & $0.95 \pm 0.02$ & $18.4 \pm 0.17$ & $2.52 \pm 0.05$ \\
rms (mm) & $0.84 \pm 2 \times 10^{-4}$ & $0.66 \pm 1 \times 10^{-4}$ & $4.57 \pm 9 \times 10^{-3}$ & $3.74 \pm 7 \times 10^{-3}$
\end{tabular}

Table 2.6: Simulation results of the positron annihilation distribution projected onto the $\mathrm{x}$-axis for both a ${ }^{22} \mathrm{Na}$ and $\mathrm{a}^{68} \mathrm{Ga}$ point source for magnetic field strengths of $0 \mathrm{~T}$ and $7 \mathrm{~T}$ in lung tissue (water scaled by one third of the density). The magnetic field is oriented in the positive $\mathrm{z}$ direction for the $7 \mathrm{~T}$ simulation results. The rms values are calculated using the magnitude of the positron range distribution. 


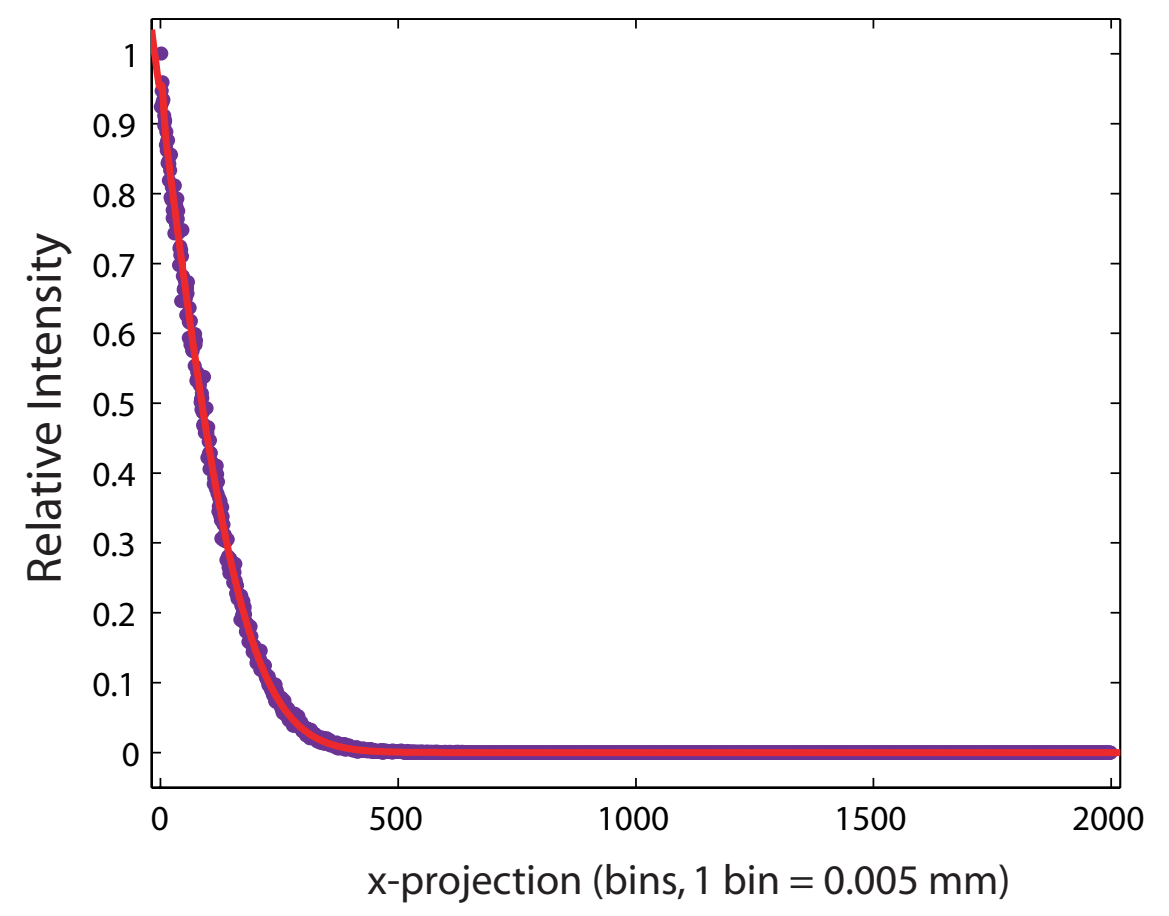

Figure 2.22: Fit of ${ }^{68} \mathrm{Ga}$ EGS4 positron range annihilation x-projection of a point source at $7 \mathrm{~T}$ in water using a standard double Gaussian fit function. The FWHM and FWTM are $0.84 \mathrm{~mm}$ and $2.28 \mathrm{~mm}$, respectively. Each bin in the plot corresponds to $0.005 \mathrm{~mm}$. 

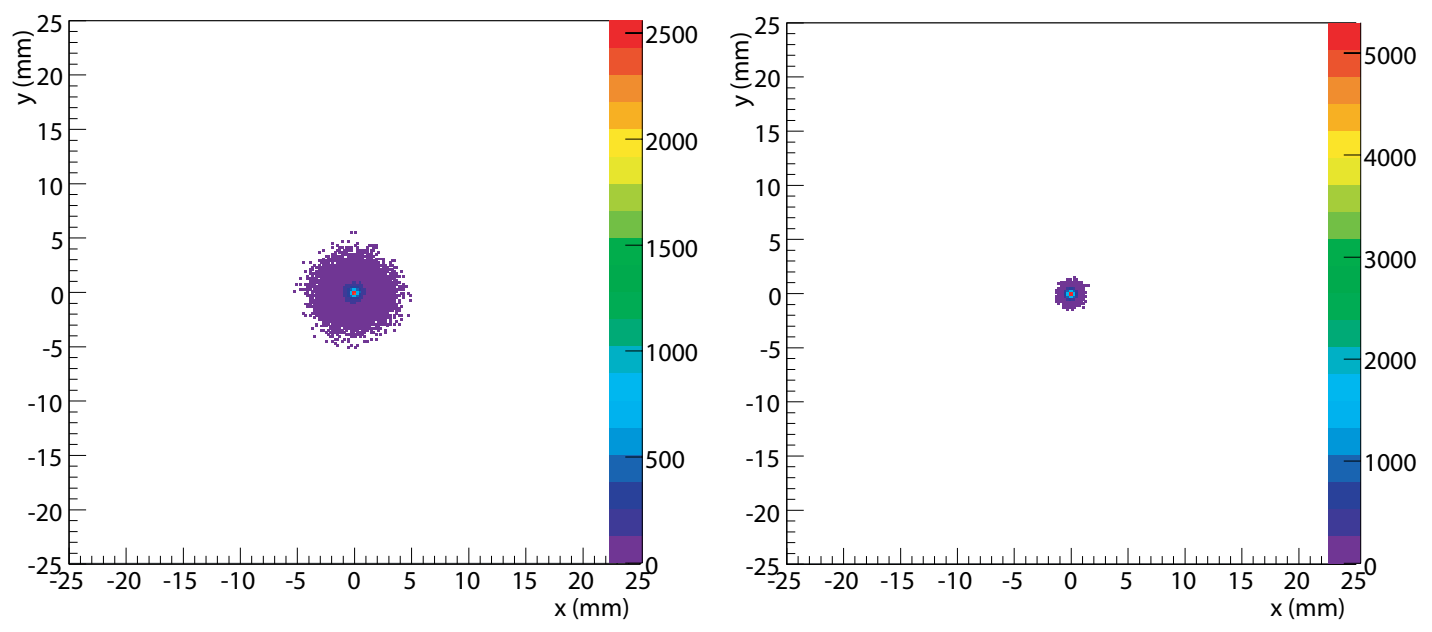

Figure 2.23: Density plots of the positron-electron annihilation positions of a ${ }^{22} \mathrm{Na}$ point source in "lung" tissue (water scaled by $1 / 3$ of the density) at $0 \mathrm{~T}$ (left) and with a $7 \mathrm{~T}$ magnetic field applied transverse to the plane (right).
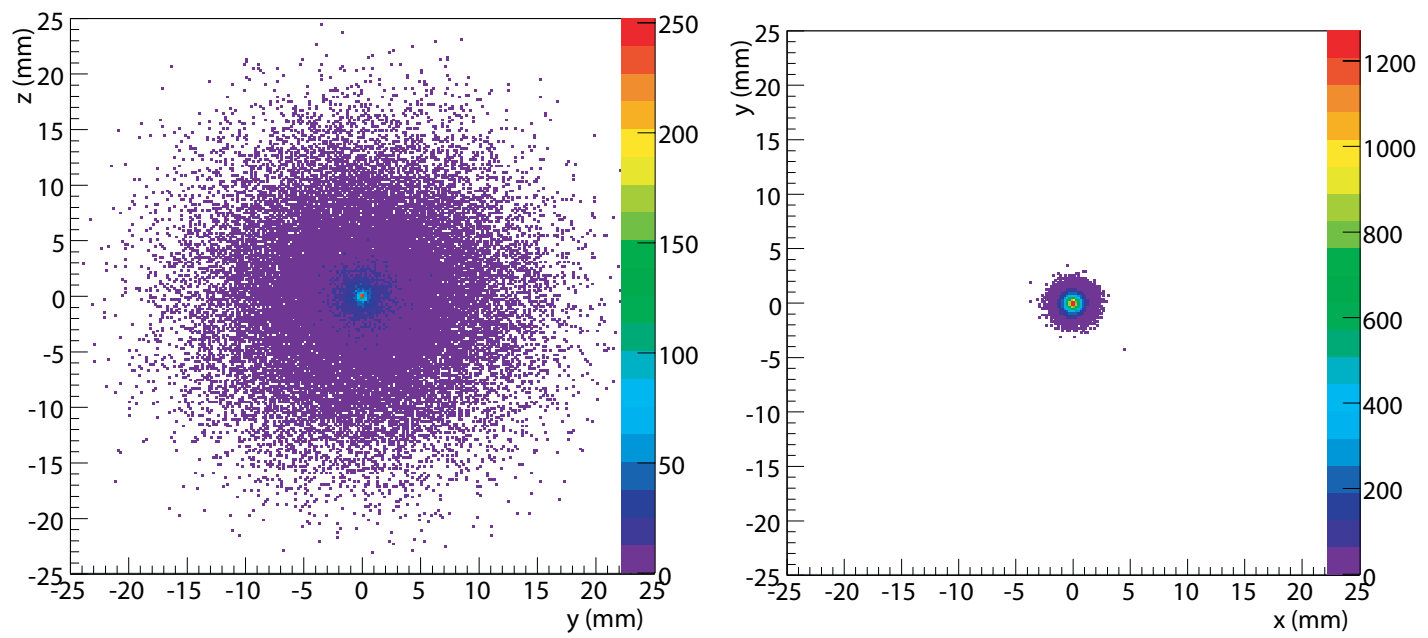

Figure 2.24: Density plots of the positron-electron annihilation positions of a ${ }^{68} \mathrm{Ga}$ point source in "lung" tissue (water scaled by $1 / 3$ of the density) at $0 \mathrm{~T}$ (left) and with a $7 \mathrm{~T}$ magnetic field applied transverse to the plane (right). 


\subsection{Photon Interactions with matter}

Photons interact with matter mainly through Compton scattering, photo-electric absorption, pair production, and Rayleigh scattering. Figure 2.25 displays the relevant interaction cross-sections of photons in silicon versus incident photon energy (excluding Rayleigh scattering) [33]. The red line in the diagram represents the energy

of the annihilation photons. The dominant physical interaction for $511 \mathrm{keV}$ photons passing through silicon is Compton scattering. Ninety-nine percent of all $511 \mathrm{keV}$ photon interactions in silicon will be Compton scattering events. The final 1 percent is undergo Rayleigh scattering with very few photo-electric interactions. Pair production is not possible because $511 \mathrm{keV}$ photons do not possess enough energy to create an electron-positron pair. In this section, each of the above particle interaction are discussed along with the EGS4 modeling of these processes. The following sections assume silicon as the scattering medium.

\subsubsection{Compton Scattering}

The Compton scattering interaction occurs when a photon scatters off an electron transferring a portion of its energy and momentum in the process. Both particles scatter in different directions as shown in Figure 2.26. Using energy and momentum conservation and assuming that the recoil electron is at rest yields the following equation for the scattered photon energy:

$$
E_{\gamma}^{\prime}=\frac{E_{\gamma}}{1+E_{\gamma}(1-\cos \theta) /\left(m_{0} c^{2}\right)}
$$

where $E_{\gamma}^{\prime}$ is the scattered photon energy, $m_{0}$ is the rest mass of the electron, and $\theta$ is the photon scattering angle. 


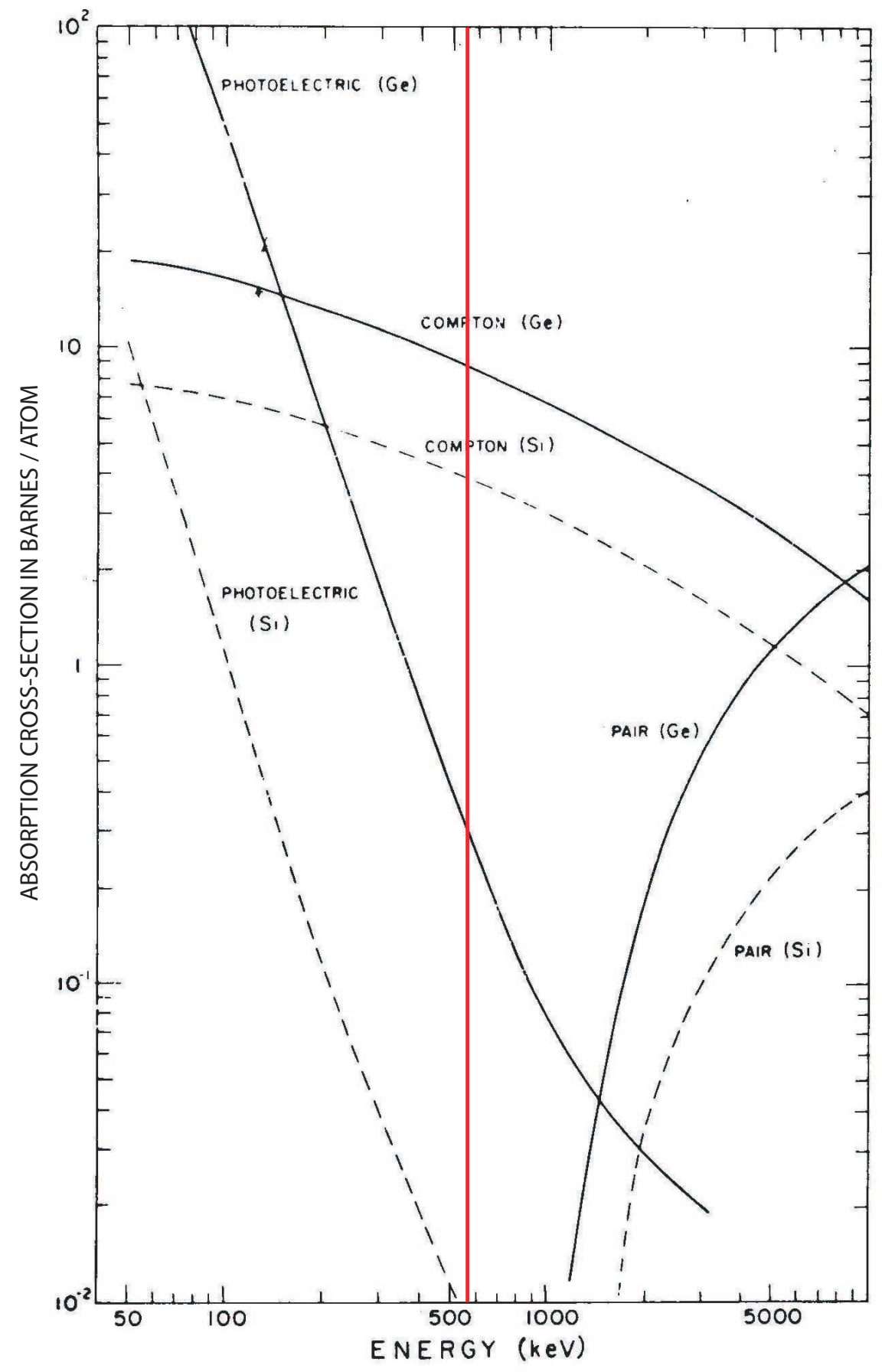

Figure 2.25: The absorption cross-section versus energy for the photo-electric effect, Compton scattering, and pair production for Si and Ge [33]. 
Equation 2.31 assumes that the electron is initially at rest. However, this assumption is not true in this study because the majority of Compton scattering events will be with atomically bound electrons. The non-zero momentum of the electron will give rise to an angular deviation from the expected scattering angle that assumed the electron was initially at rest. The deviations are on the order of 1.0 degrees FWHM for small scattering angles and up to 10 degrees FWHM for backscattered events using $511 \mathrm{keV}$ incident photons in silicon [34]. The uncertainty in the Compton scattering angle due to the non-zero momentum of the electron is known as Doppler broadening.

Although the scattered photon is not used in this experiment, it could yield useful information if it is detected by a secondary, outer PET ring. For example, the energy of the recoil electron in the silicon detector and the energy of the scattered photon recorded in the PET ring can be used to reduce scattered coincidence events by demanding that the energy of the two detectors sum to $511 \mathrm{keV}$. Additionally, the energy and position of the scattering event in the silicon and the energy and position of the scattered photon absorbed in the secondary detector can be used to back-project a cone into the image space through the use of the Compton scattering equation, thus placing additional constraints on the probable annihilation position [35] [36].

In this study the energy of the recoil electron is detected, not the energy of the scattered photon. The energy of the recoil electron is given simply by energy conservation where the energies of the post-scattered particles are primed.

$$
E_{\text {electron }}^{\prime}=E_{\gamma}-E_{\gamma}^{\prime}
$$

Figure 2.27 is a plot of equations 2.31 and 2.32. This plot shows the energy of both the scattered photon (black line) and the recoil electron (red line) versus the photon scattering angle. 


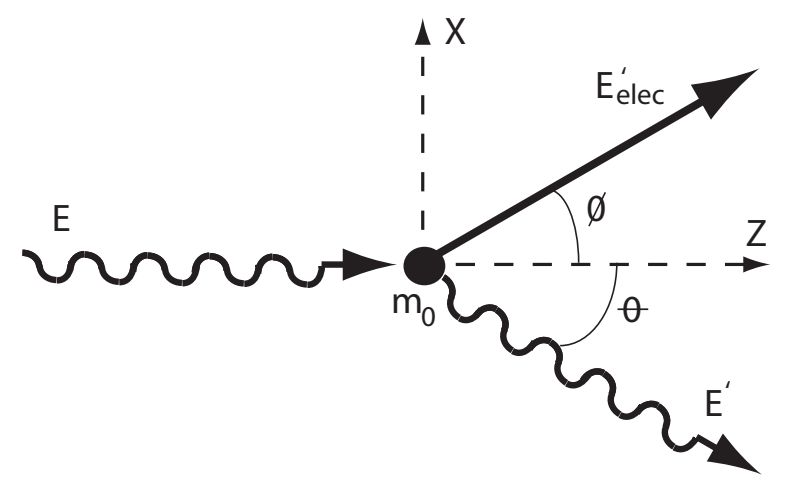

Figure 2.26: A diagram of the Compton scattering process.

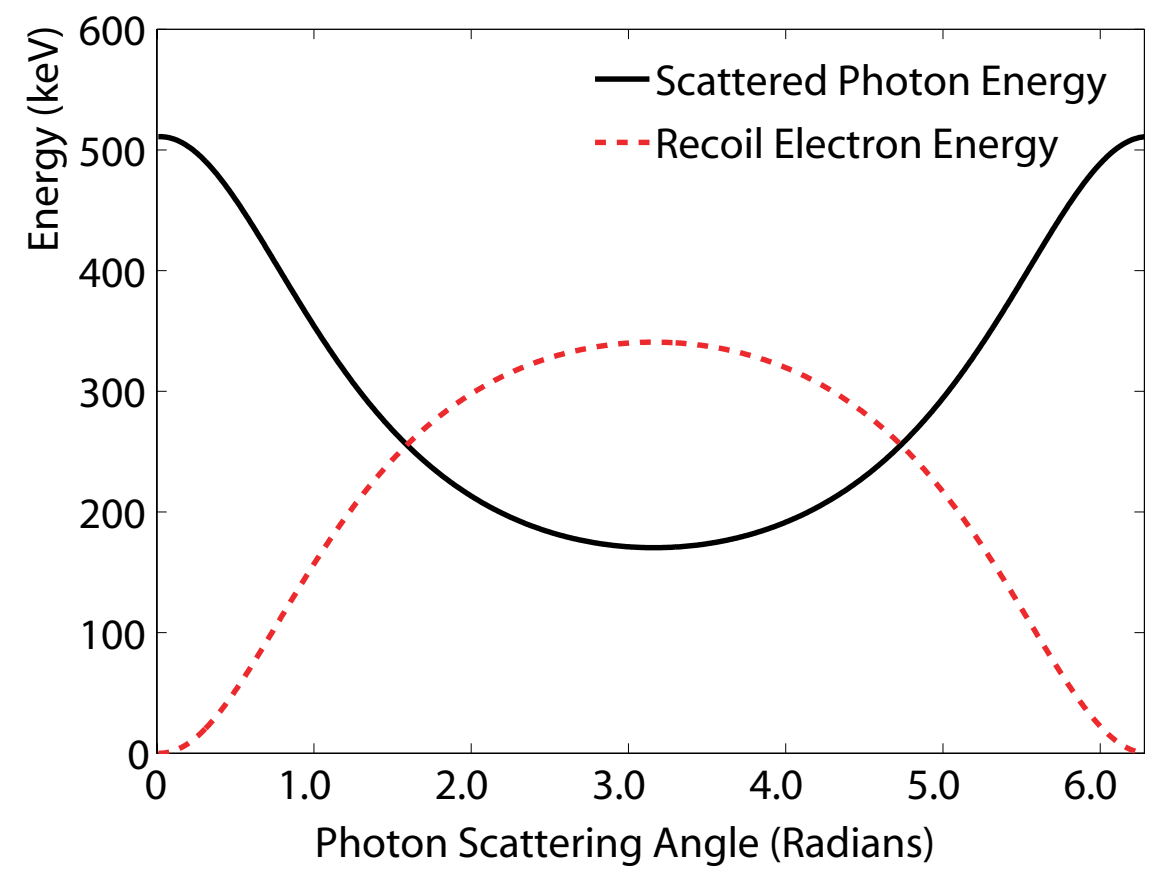

Figure 2.27: The energy of both the scattered photon and recoil electron energies versus the photon scattering angle for $511 \mathrm{keV}$ incident photons. 
EGS4 uses the Klein-Nishina differential scattering cross section to determine the scatting angle of the photon when a Compton scattering event occurs. The KleinNishina formula is given by [6]:

$$
\frac{d \sigma}{d \Omega}=Z r_{0}^{2}\left(\frac{1}{1+\alpha(1-\cos \theta)}\right)^{2}\left(\frac{1+\cos ^{2} \theta}{2}\right)\left(1+\frac{\alpha^{2}(1-\cos \theta)^{2}}{\left(1+\cos ^{2} \theta[1+\alpha(1-\cos \theta)]\right.}\right)
$$

where $\alpha=E_{\gamma} / m_{0} c^{2}$ and $r_{0}$ is the previously defined classical electron radius. Figure 2.28 is a plot of the probability that a $511 \mathrm{keV}$ photon will scattering into angle $\theta$ in silicon, $Z=14$. This plot was generated by integrating equation 2.33 in 0.01 radian steps over the angle $\theta$. The area under the curve is normalized to one. This plot shows that the most probable Compton scattering events for $511 \mathrm{keV}$ photons traveling through silicon are small angle photon scatters resulting in low energy recoil electrons, while photon backscattering events which transfer the most energy to the recoil electrons are the less probable.

Figure 2.29 shows the energy spectrum of the recoil electron for one million events using $511 \mathrm{keV}$ incident photons simulated in EGS4. The sharp peak at $340 \mathrm{keV}$ is known as the Compton edge which is the maximum energy that can be transfered to an electron from the incident photons. The Compton edge corresponds to a Compton scattering events were the photon is completely backscattered. The sharp peak of the Compton edge is a clear demonstration the Doppler broadening is not included in the EGS4 simulation.

\subsubsection{Coherent Rayleigh Scattering}

Rayleigh scattering occurs when a photon scatters off of a tightly bounded inner atomic electron and fails to transfer a sufficient amount of energy to ionize the atom. 


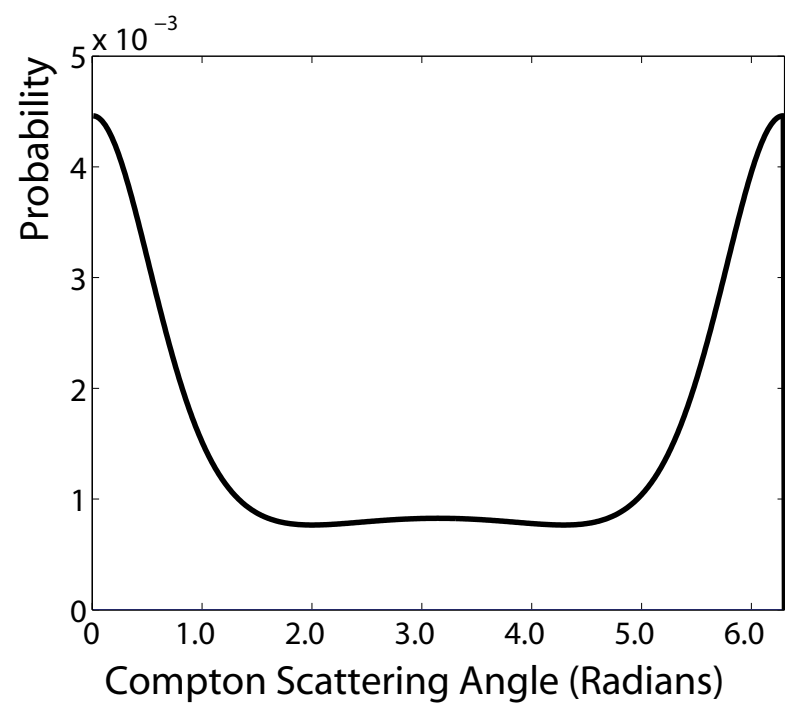

Figure 2.28: Plot of Klein-Nishina scattering probability for silicon using $511 \mathrm{keV}$ incident photons. The area under the plot is normalized to one.

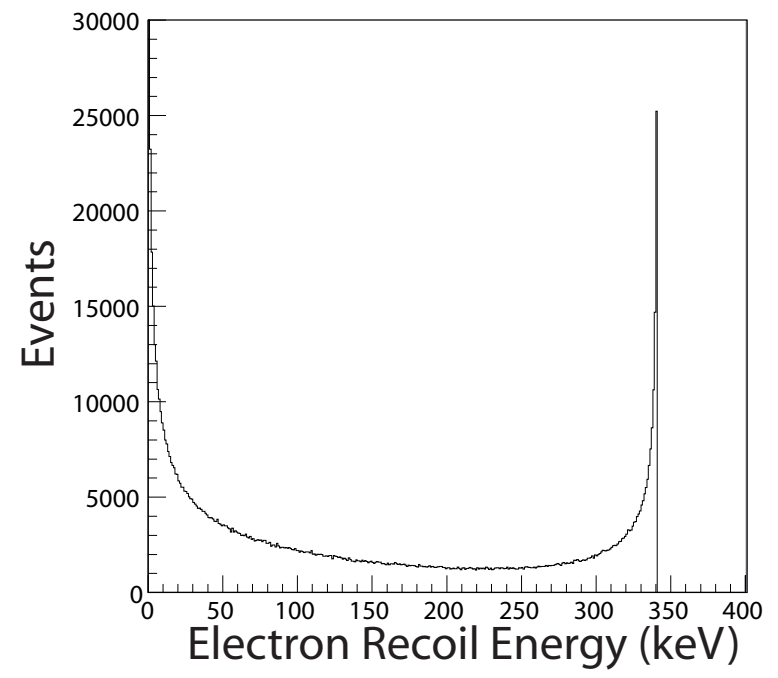

Figure 2.29: Energy spectrum of the Compton recoil electrons using $511 \mathrm{keV}$ incident photons for one million events in silicon simulated in EGS4. 
As electrons in the atom are only allowed to be at discrete energy levels, the atom, as a whole, must absorb the transfered energy and momentum of the scattering event. The mass of an atom is much greater than the mass of an electron. By replacing $m_{e}$ in equation 2.31 with the mass of the atom $M_{a}$ one can see that the energy of the scattered photon is essentially unchanged, thus this scattering process is essentially elastic. In Rayleigh scattering the deflection of the photon scattering angle is highly peaked around zero degrees. The probability of Rayleigh scattering increases with atomic $\mathrm{Z}$ because of the increased number of tightly bound electrons in the atom.

The effects of Rayleigh scattering are negligible in this experiment for a number reasons. The first reason is that the cross section for Rayleigh scattering is small in comparison to Compton scattering in silicon due to silicon's low atomic number and the energy of the incident annihilation photons. Secondly, the energy a photon loses during the scattering process is negligible and the scattering angle is strongly peaked around zero degrees [1]. Rayleigh scattering is not a dominant physical effect for 511 keV photons scattering in silicon.

\subsubsection{Photo-electric Absorption}

Photo-electric absorption occurs when an incident photon transfers all of its energy to an atomically bound electron. This electron is ejected from the atom with the energy of the incident photon minus energy binding the electron to the atom. This electron is known as a photoelectron. The vacancy left by the photoelectron is quickly filled by an electron from a higher energy level, emitting a X-ray photon in the process. The photo-electric effect is negligible for the energy range of gamma rays used in PET. 


\subsubsection{Pair Production}

Pair production occurs when a high energy photon is transformed into an electronpositron pair in the presence of the Coulomb field of the nucleus. As the electron and positron both have a rest mass of $511 \mathrm{keV}$, the incident gamma ray must possess at least twice the energy of the rest mass of the electron for a pair production interaction to occur. The photons used in PET only possess half the energy necessary to create an electron-positron pair. Pair production events never occur in PET systems.

\subsection{Full EGS4 simulation}

A full EGS4 simulation of the experimental set-up was performed in order to compare the images of the real data to simulated data. A block diagram of the full simulation process is shown in Figure 2.30.

The first stage simulates the positron range distribution and writes the $\mathrm{x}, \mathrm{y}, \mathrm{z}$ annihilation positions to a file. In our experiment, point sources of radii $0.25 \mathrm{~mm}$ and $1.0 \mathrm{~mm}$ are used for the ${ }^{22} \mathrm{Na}$ and ${ }^{68} \mathrm{Ga}$ point sources, respectively. In order to simulate these point sources, random positions are picked within the diameter of each source on an event-by-event basis. These random positions are distributed uniformly throughout the source. A positron is emitted from each randomly selected location in the source in a random direction and with a random energy consistent with the positron energy spectrum of the radioisotope under study as detailed in section 2.3. The positron is tracked through the material until an annihilation event occurs as described in section 2.4. The $\mathrm{x}, \mathrm{y}, \mathrm{z}$ annihilation position for each event is written to a text file for use in the second half of the EGS4 simulation. 


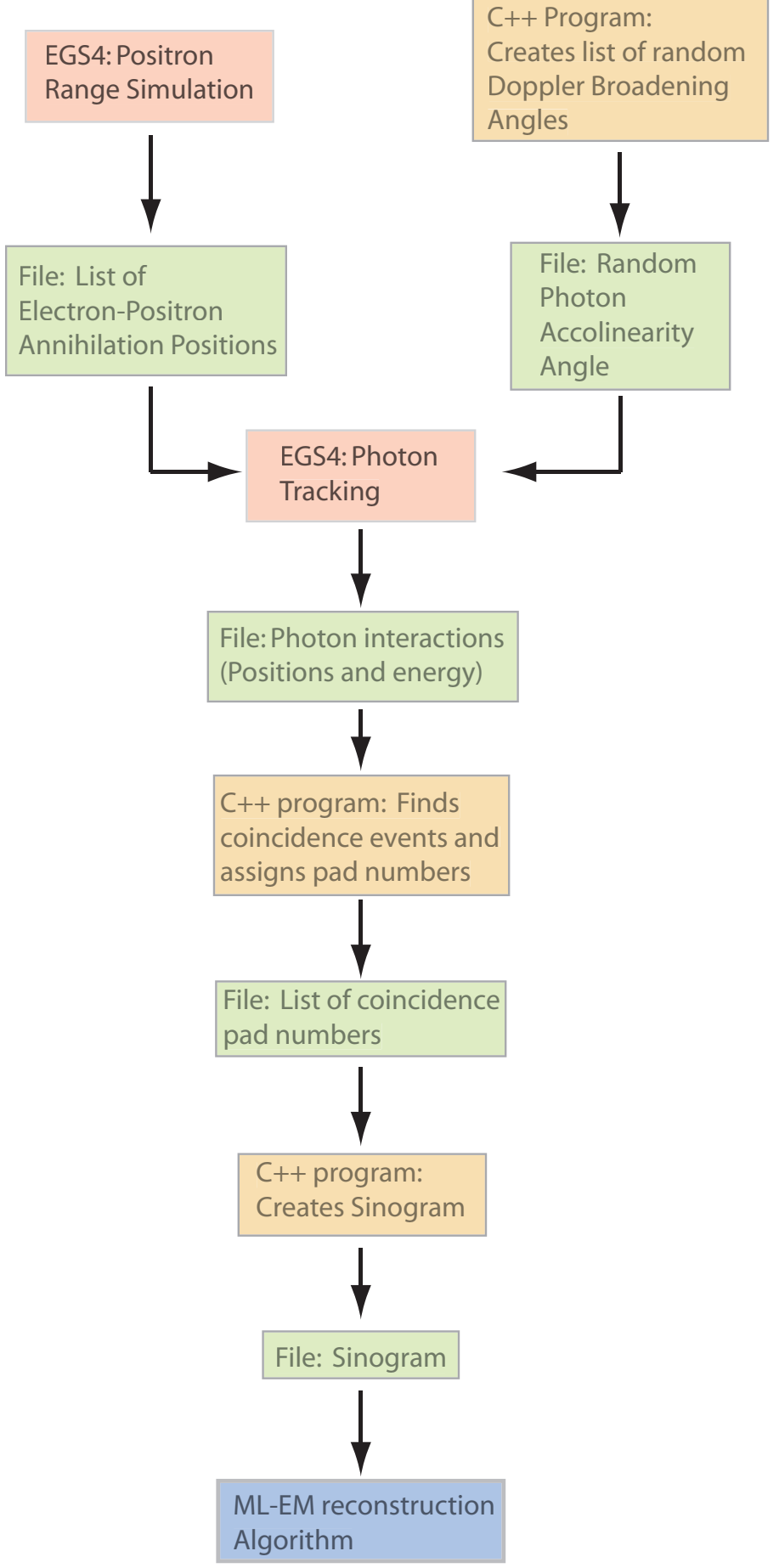

Figure 2.30: Block diagram of total simulation. 
The annihilation positions are used as the input for the second stage of the simulation which tracks the two $511 \mathrm{keV}$ annihilation photons from the annihilation positions through the air surrounding the source and through the two silicon detectors. The two photons are skewed from their ideal 180 degree scattering angle by a random acollinearity angle consistent with the effect of Doppler broadening in water as discussed in section 2.6. In order to reduce processing time the annihilation photon pairs is emitted entirely within the plane of the detectors, and the photon emission angle is chosen randomly from a set of angles that will intersect the two silicon detectors. All photon interactions are written out to a text file which is later scanned for coincidence events between the two silicon detectors. When both photons interact with their respective silicon detectors during a single event, a coincidence has occurred and the position of the scattering interaction and the energy of the scattered photon are written to a file. The energy transfered to the recoil electron can easily be calculated using conservation of energy. However, as discussed earlier, the energy of the recoil electron is not used in this experiment, only the position information is used.

In order to simulate a full detector ring, the source is rotated, and data is collected at discrete angular intervals (see Fig. 2.31). Rotating the source is analogous to rotating the detectors around the source for symmetric source distributions. However, when a magnetic field is applied the symmetry of the source distribution is broken and rotating the source is no longer analogous to rotating the detectors. The reason, as shown in Fig. 2.32, is that the source distribution is not static for each rotation angle; therefore, the detectors will only "see" the unconfined or restricted positron range depending on the orientation of the silicon detectors with respect to the magnetic 


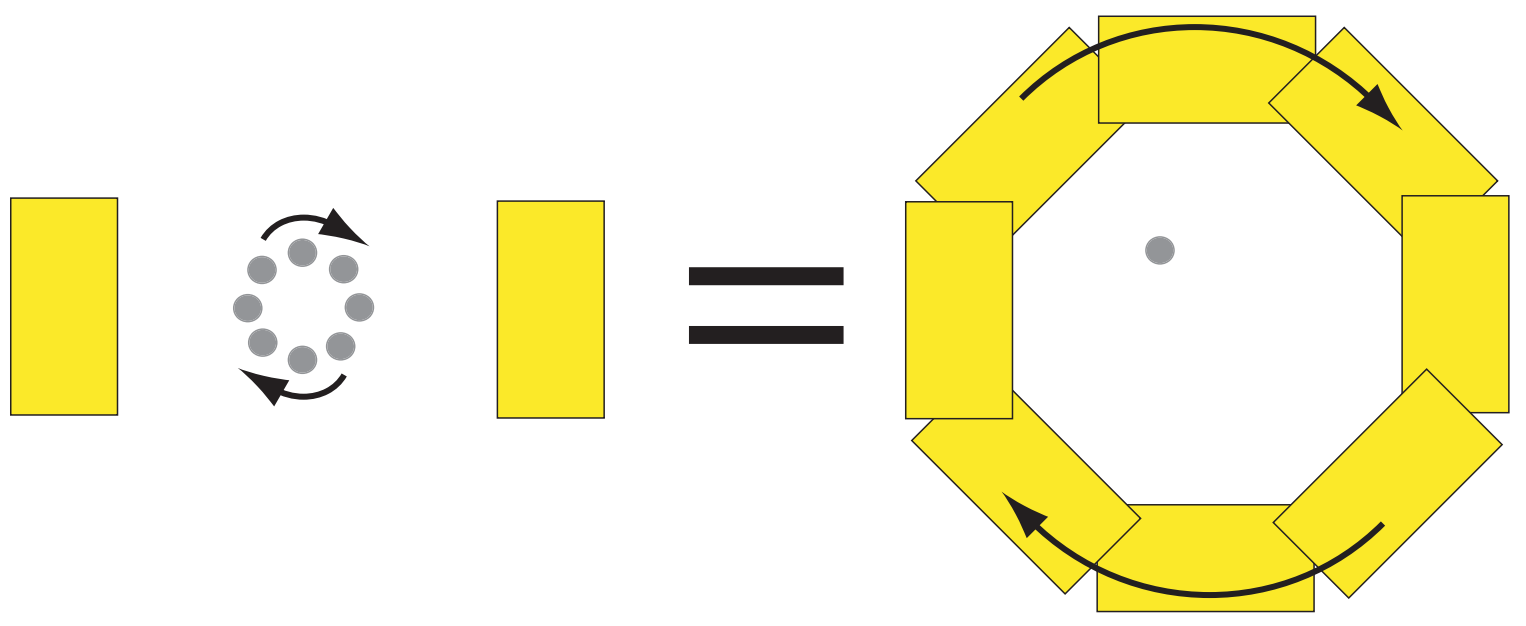

Figure 2.31: An illustration of the equivalence of rotating a symmetric positron source between two stationary silicon detectors (Left) and rotating the silicon detectors around a stationary symmetric positron source (Right).

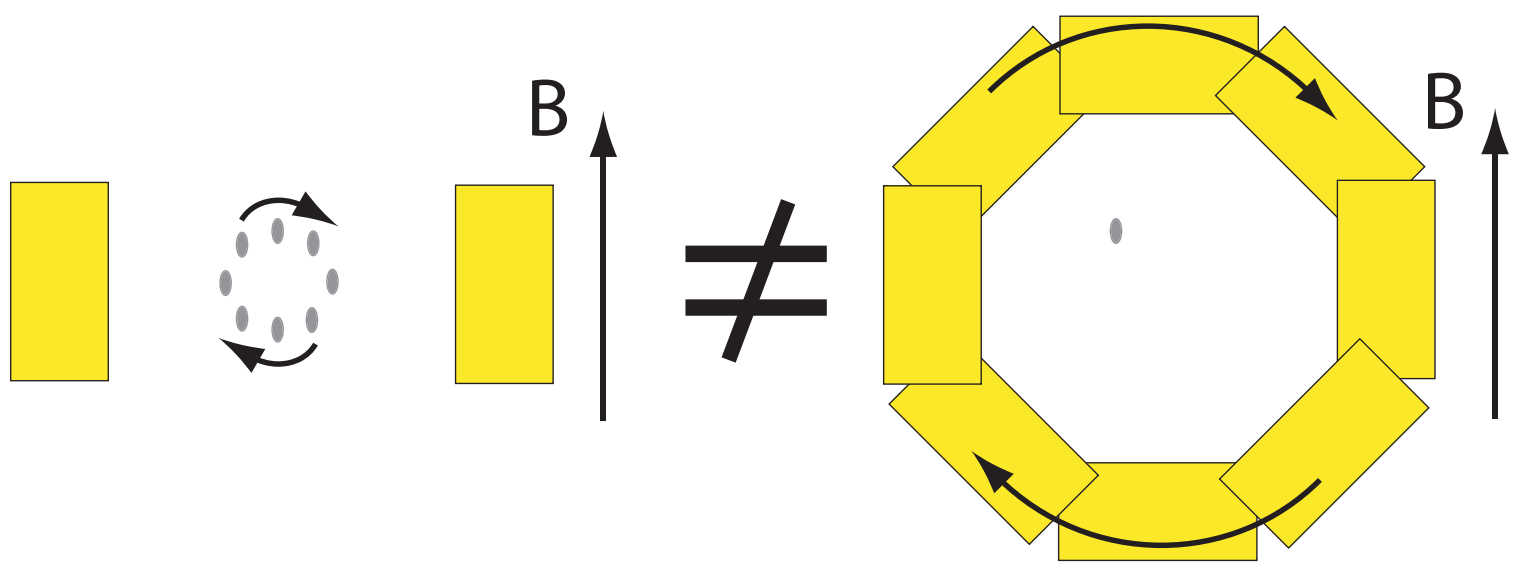

Figure 2.32: An illustration that rotating the source between two stationary silicon detectors (Left) is not equivalent to rotating the detectors around a stationary, nonsymettric source (Right). 
field vector. Thus, for the majority of our study, we will focus on symmetric source distributions. The $0 \mathrm{~T}$ measurements are always symmetric, as are the measurements with the image plane transverse to the magnetic field. For our simulations (and experimental measurements) data was collected in eighteen, 20 degree steps. This step size is sufficient for full angular sampling of the data.

After the EGS4 simulation of the photon transport is completed for each angular step, a look-up-table relating silicon position to silicon pad number is consulted to see which pad the event occurred in. For real experimental data the actual location of the Compton scattering events is not known. It is only known in which pad the event occurred. Thus, the pad position must be used to reconstruct the image instead of the known (simulated) interaction position to get a true depiction of the expected image resolution. Each scattering position is assigned a random position in the interaction pad. The input to the ML-EM reconstruction algorithm are the back-projected LORs of these positions stored in a 2D matrix, known as a sinogram. The details of both sinogram creation and image reconstruction are described in chapter 5. The results of the full EGS4 simulation are compared to the experimental measurements in chapter 6 . 


\section{CHAPTER 3}

\section{Silicon Detectors}

Silicon detectors and their associated readout electronics can be used to detect both the energy transfered to the recoil electron by the annihilation photon during a Compton scattering event and to determine the position of the interaction. This chapter first discusses the reasons that silicon detectors are ideal for this study followed by a description of their basic operational principles. Finally, the physics and readout electronics that limits the energy, position, and timing resolutions is presented along with the operational performance of the two silicon detectors used in this experiment.

\subsection{Why use silicon as a PET detector?}

The ideal characteristics of a PET detector include high efficiency, excellent energy, spatial, and timing resolutions, preferably, at a low cost. In practice, it is difficult to find a detector material that excels at every relevant characteristic beneficial to PET systems. Thus, depending on the goals of a particular imaging system, compromises are made to produce the best possible system. For this experiment there is the additional constraint that the PET detector must be able to operate properly in a 
strong magnetic field. This is the main constraint that dominates the decision of the detector material used in this study.

Conventional PET scanners, composed of a combination of scintillation crystals and arrays of PMTs, are not the ideal detector system for this experiment because the PMTs will not function properly in strong magnetic fields. This is due to both the interruption of the electron cascade process caused by the deflection of the electrons by the magnetic force and the possible magnetic forces on the PMT components themselves. This is not to say that conventional scintillators can not be used in strong magnetic fields. In fact, light guides from highly segmented scintillation crystals can be used to guide the scintillation light out of the high magnetic field into a region where the PMTs can perform the signal amplification process. For a high resolution system, the scintillation crystals must be segmented into elements that are long (for sensitivity) with a small area face (for high resolution LOR back-projections). Not only is the segmented scintillator expensive, but the resolution will also suffer due to parallax errors [37]. Although conventional PET systems can be made to operate in strong magnetic fields, it is both more complex and costly to modify conventional PET detectors to operate under these conditions than it is to use a detector that will function in a magnetic field unmodified.

In contrast to scintillator/PMT systems, solid state detectors can operate in strong magnetic fields with little to no modifications (as opposed to the expensive light guide solution). Potential candidates include detectors made from silicon, germanium, or cadium zinc telluride (CZT). All of the above solid-state detectors can be constructed with small detection elements on the order of a millimeter and thus all possess excellent spatial resolutions. Both Ge and CZT will possess a higher sensitivity than 
Si by a factor of two due to their larger attenuation coefficient (see Table 1.1). The energy resolution of each detector type is comparable because they all produce a similar number of charge carriers per unit of deposited energy. The draw back that disqualifies Ge as a detector material for this experiment is the need to cryogenically cool the detector in order to reduce noise caused by leakage currents [6]. CZT is less desirable than silicon due to its poorer timing characteristics resulting from its slower free charge carriers. Although timing in this particular experiment is not a crucial component, future systems imaging higher rate sources will demand a shorter time window in order to reduce random coincidence events. For these reasons, despite a lower efficiency, silicon is the ideal detector material for this application. It is worth mentioning, although it is not important for this application, that $\mathrm{Si}$ also possess a smaller Doppler broadening uncertainty due to its lower atomic number Z in comparison to Ge and CZT. A smaller Doppler broadening uncertainty is important for both Compton camera applications [36] and Compton PET geometries [35].

\subsection{Basic Silicon Detector Operation}

The silicon detectors in this experiment are used to detect the positions of the Compton scattering interactions of the two $511 \mathrm{keV}$ annihilation photons. During the Compton scattering process, a photon transfers a portion of its energy and momentum to an electron in the silicon, and then scatters off the electron at an angle with reduced energy consistent with the Compton scattering equation 2.31. During the scattering process, the incident photon transfers enough energy to an atomically bound electron to raise the electron from the valence to the conduction band, typically with enough kinetic energy to raise additional loosely bound electrons into the conduction band. 


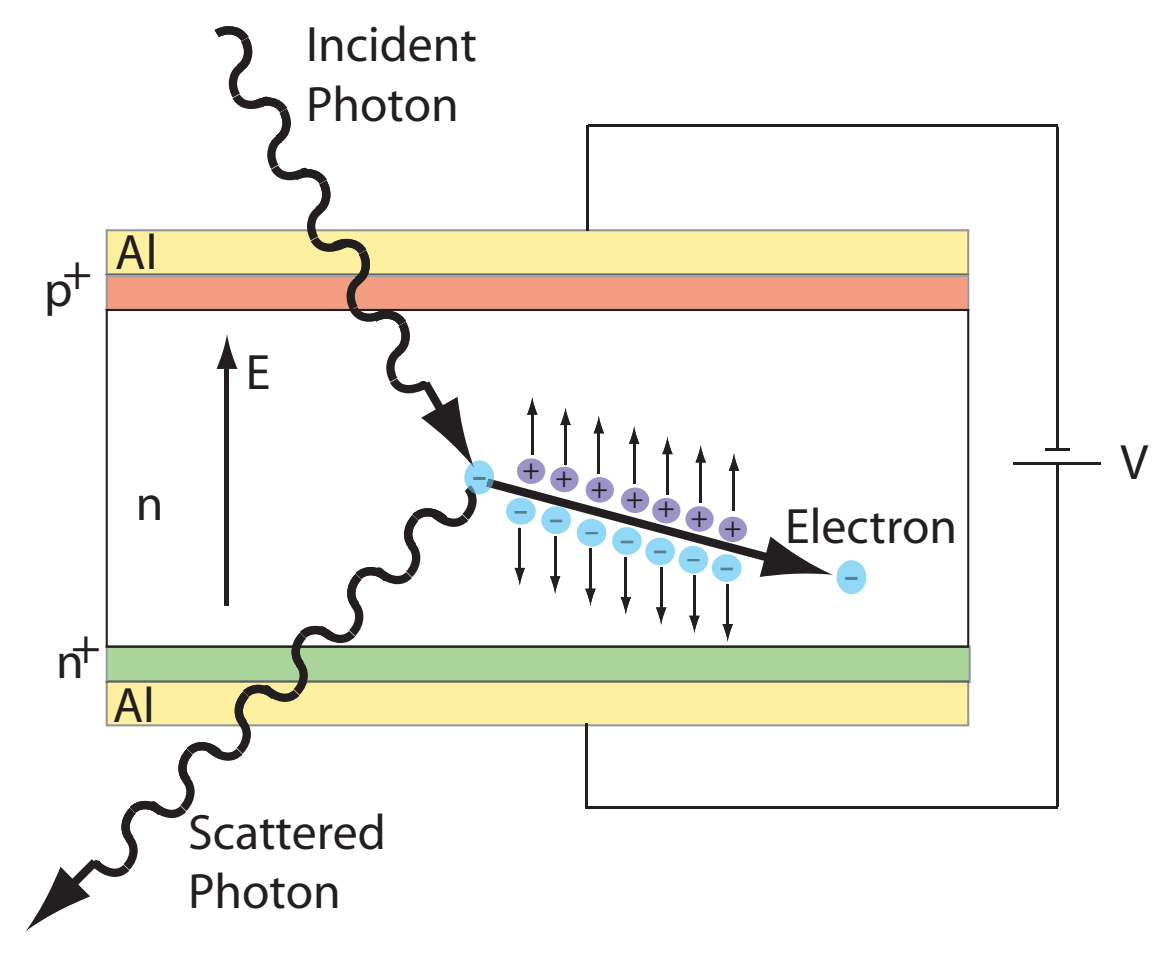

Figure 3.1: Diagram of the basics of silicon detector operations. Incident gamma radiation liberates a bound electron with sufficient energy that it ionizes additional atoms, creating a surge of electron-hole pairs. The number of free charge carriers is directly proportional to the initial kinetic energy of the recoil electron. The free charge carriers are swept out of the depletion region by an electric field created by a reverse bias voltage. The electrons and holes are collected at either end of the silicon detector volume and form the basis for determining the energy and position of the photon interaction. 
The energy level in the silicon atom that previously contained the liberated electron is now left with a vacancy, termed a hole. The electron-hole pair constitute the initial charge carriers in the surge of particles to follow.

The liberated electron traveling through the silicon will transfer its kinetic energy into the creation of electron-hole pairs. Each secondary electron (if sufficiently energetic) will continue to create additional electron-hole pairs. This process will continue until the secondary electrons do not possess enough kinetic energy to create additional electron-hole pairs $(3.6 \mathrm{eV})$. The total number of electron-hole pairs created in the shower is directly proportional to the energy transfered to the recoil electron in the Compton scattering process.

The electron-hole pairs must be completely collected in order to accurately record the energy deposited in the silicon detector and the position of the interaction. The charges are collected by an electric field created by the application of a reverse bias voltage applied across the detector thickness. The electric field extends the depletion region, a volume devoid of free charge carriers, between the $p$ and $n$ doped regions of the silicon detector. The electric field separates the electrons and holes and directs them to opposite sides of the detector volume where they are collected by the electrodes. Once the surge of current is collected, it is amplified by low noise front-end electronics. The resulting electric pulse can be used to determine the initial energy of the recoil electron and to produce a trigger signal if the energy of the pulse exceeds the threshold voltage. In this experiment, the trigger signal is used in conjunction with the trigger signal from the second silicon detector to determine if a coincidence event has occurred. 
The remainder of this chapter focuses on the physics of the silicon detectors used in this study, along with the associated low noise front-end readout electronics. The performance characteristics of the two silicon detectors is also presented.

\subsection{Silicon Detector Physics}

In practice, intrinsic (pure) solid-state detectors are impossible to achieve. Often the semiconducting materials are doped with impurities to give a detector various properties depending on the application. The two types of doping used in semiconductors are $p$ and $n$ type doping. In both types of doping, impurities are purposely introduced into the silicon lattice structure in small concentrations (typically a few impurity atoms per one million silicon atoms). For $p$ type semiconductors the impurity atoms have one less valance electron than the surrounding silicon atoms, essentially introducing a hole into the bulk structure. For $n$ type semiconductors the impurities atoms introduce an extra electron that is only loosely bound to the impurity atom. For every impurity atom introduced to the silicon lattice an extra hole ( $p$ type) or electron ( $n$ types) is present in the silicon.

The element used to dope the semiconductor during the growing process can be switched in order to change the properties of the semiconductor. This technique is used to create a continuous junction between $p$ and $n$ type semiconductors, termed a $p n$ junction. The $p n$ junction is the key to particle detection. To illustrate this point, first consider the physics that will occur due to the difference of the dopant concentrations in the two regions. Due to the diffusion of free charge carriers into regions of lesser concentrations the net excess of holes from the $p$ type region will diffuse into the $n$-type region. Analogously, the excess electrons from the $n$-type 
region will diffuse into the $p$-type region. The electrons diffusing into the $p$ side of the junction will combine with holes in the $n$ side, while the holes diffusing across the junction will combine with electrons on the $n$ side. The free electrons from the $n$ region recombining with holes in the $p$ region form stationary negative ions in the $p$-type region while leaving behind stationary positive ions in the $n$-type region. The net effect of this diffusion and recombination of electrons and holes is the creation of a region free of charge, the depletion region. A potential difference will exist across the depletion region that gives rise to an electric field that eventually stops the diffusion of free charges across the boundary. The depletion region is used for particle detection.

The thickness of the depletion region can be increased by the application of a reverse bias voltage as shown in Fig. 3.1. The thickness of the depletion region is given by

$$
d=\sqrt{\frac{2 \epsilon \epsilon_{0} V}{e_{0} N_{d}}}
$$

where $\epsilon=12.0$ is the dielectric constant of silicon, $\epsilon_{0}$ is the permittivity of free space, $V$ is the reverse bias voltage, $e_{0}$ is the charge of the electron, and $N_{d}$ is the number of donor atoms. The number of donor atoms can be calculated using the equation

$$
N_{d}=\frac{1}{\rho \mu_{e} \epsilon_{0}}
$$

where $\rho$ and $\mu_{e}$ are the resistivity and electron mobility in silicon, respectively. The resistivity of the silicon used in this study is $\rho=20 \mathrm{k} \Omega \mathrm{cm}[34]$ and $N_{d}=2.3 \times 10^{17}$.

The full depletion voltage $V_{F D}$ is found by replacing $d$ with the detector thickness $w$ and solving for the voltage in equation 3.1. The thickness of the silicon detectors used in this experiment are $w=1.0 \mathrm{~mm}$. Using the value for $N_{d}$ calculated above and 
a detector thickness of $1.0 \mathrm{~mm}$, the theoretical full depletion voltage for our detectors is $190 \mathrm{~V}$.

The electric field in the depletion region allows the free charge carriers liberated by the recoil electron to be guided to the electrodes and collected. The physics of the electron-hole propagation through the detector material will directly affect the timing, energy, and position resolutions of the detector. The following three sections discuss the physics responsible for the position, timing, and energy resolution of silicon detectors.

\subsubsection{Position Resolution}

The position resolution is ultimately limited by the diffusion of the electrons and holes from the original photon interaction position. Each electron that is raised to the conduction band during the shower creation process possess kinetic energy and will scatter through the silicon analogous to the positron scattering through water. The range of the electron as it scatters through the material sets a lower limit on the spatial resolution of the detector. The maximum energy that can be transfered from a $511 \mathrm{keV}$ photon to an electron is $340 \mathrm{keV}$ (corresponding to the Compton edge). The average range for a $340 \mathrm{keV}$ electron in silicon is $0.550 \mathrm{~mm}$ [34]. The dimensions of the silicon detectors used in this experiment are $1.4 \mathrm{~mm} \times 1.4 \mathrm{~mm}$ in area and 1.0 mm thick. For Compton scattering events that take place near a pad boundary charge can leak into neighboring pads. The charge sharing between silicon pads can be used to determine a more accurate determination of the Compton interaction. However, for the majority of events the free charge carriers will be confined to a single pad. 
The broadening of the charge distribution will increase as a function of time until all of the original recoil electron's energy has been transformed into a cloud of electronhole pairs. A cross section through the center of the distribution is Gaussian with a standard deviation given by [6]:

$$
\sigma=\sqrt{2 D t}
$$

where $t$ is the time in seconds and $D$ is the diffusion coefficient given by

$$
D=\mu \frac{k T}{e}
$$

where $\mu$ is the mobility of the charge carrier, $k$ is the Boltzman constant, $T$ is the temperature measured in Kelvin, and $e$ is the charge of the electron.

For this experiment the position resolution is dominated by the size of the pad and not by the range of the recoil electron.

\subsubsection{Timing Resolution}

The timing resolution is limited by both how quickly the electron-hole pairs can be collected and the response of the front-end electronics to determine if an event has occurred above threshold. This section addresses the physics affecting the timing resolution for the silicon detectors used in this study.

Variations in charge collection times appear due to the depth of the interaction in the silicon, the difference in mobility of the electrons and holes, and the variation in the electric field strength spanning the depletion region. The time it takes for the charges to travel from the Compton scattering position to the charge collecting electrode is determined by the mobility of the electrons and holes and by the strength of the electric field in the depletion region. This effect is quantified by equations 3.5 
and 3.6

$$
\begin{gathered}
v_{e}=\mu_{e} E(x) \\
v_{h}=\mu_{h} E(x)
\end{gathered}
$$

where $E(x)$ is the magnitude of the electric field as a function of position, $\mu_{e}$ and $\mu_{h}$ are the mobility of the electrons and holes, respectively. At room temperature $(300 \mathrm{~K})$, the mobility of the electrons and holes is $1350 \mathrm{~cm}^{2} / \mathrm{V} \cdot \mathrm{s}$ and $480 \mathrm{~cm}^{2} / \mathrm{V} \cdot \mathrm{s}$, respectively [6]. The magnitude of the electric field in the depletion region between a $p^{+} n$ junction as measured from the junction is given by

$$
E(x)=-\frac{V}{w}-\frac{e_{0} N_{d}}{\epsilon \epsilon_{0}}\left(\frac{w}{2}-x\right)
$$

where $V$ is the reverse bias voltage and $w$ is the thickness of the detector.

The collection time for electrons and holes swept out of the depletion region by the electric field as a function of interaction depth $x$ is given by

$$
\begin{aligned}
& t_{c}^{e}=\tau_{e} \ln \left(\frac{3+V / V_{F D}}{1+V / V_{F D}+2 x / w}\right) \\
& t_{c}^{h}=\tau_{h} \ln \left(\frac{1+V / V_{F D}}{1+V / V_{F D}-2 x / w}\right)
\end{aligned}
$$

where $V_{F D}$ is the full depletion voltage and $\tau_{i}=\epsilon \epsilon_{0} / \mu_{i} e_{0} N_{d}$ for either electrons or holes. These times are the total time it takes to collect all of the electrons $t_{c}^{e}$ or holes $t_{c}^{h}$. The front-end electronics used in this study trigger on the rising edge of the current pulse, thus the timing resolution of the system is dependent on the rise time of the pulses and not the total collection times shown here.

Higher reverse bias voltages yield stronger electric fields, and hence quicker collection times, but only to a point. One may falsely assume that the collection time of the electron-hole pairs can be continuously improved by increasing the reverse bias 


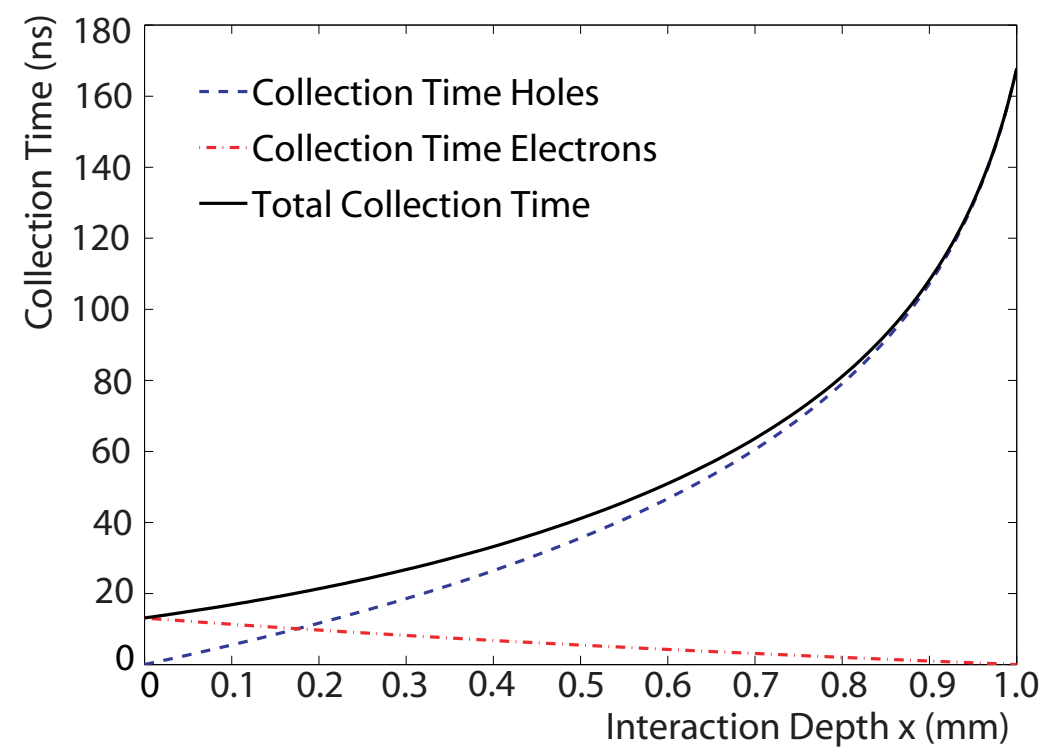

Figure 3.2: Charge collection times for a fully depleted $1.0 \mathrm{~mm}$ thick silicon detector as a function of the interaction depth. $\mathrm{x}=0 \mathrm{~mm}$ corresponds to the $p^{+} \mathrm{n}$ boundary, and $\mathrm{x}=1.0 \mathrm{~mm}$ corresponds to the $n n^{+}$boundary.

voltage; however, this is not true for two reasons. The first reason is that eventually the electron and hole drift velocities will reach a maximum value regardless of the electric field strength [38]. Secondly, too high of a voltage difference across the silicon detection volume can cause the silicon detector to break down and no longer function as a semiconductor, or can cause damage to the metallic contacts used to readout the system [6].

\subsubsection{Energy Resolution}

The energy resolution of the silicon detectors is limited by the the statistical fluctuation of the number of electron-hole pairs created during an event, variations in the leakage current, and the noise introduced by the front-end readout electronics. 
The energy resolution of any physics detector is ultimately limited by fluctuations in the number of charge carriers created per unit of deposited energy. Solid state detectors have superior energy resolutions in comparison to scintillator/PMT systems traditionally used in PET due to a much larger number of free charge carriers created per unit of deposited energy. As can be seen in Table 1.1, the number of free charge carriers created by solid-state detectors are, on average, two orders of magnitude greater than that in scintillator/PMT detectors. The advantage of having a large number of charge carriers is that the statistical fluctuation for Poisson processes is directly related to the square root of the number of detected events:

$$
\sigma(E)=\sqrt{N(E)}
$$

where $\sigma(E), N(E)=E / \epsilon$, and $\epsilon$ are the variance in the number of charges, the average number of charge pairs created for energy $E$ deposited in the detector, and the energy necessary to create a free charge carrier(s), respectively.

In solid-state detectors the variation in the number of charge pairs created at energy $E$ is less than predicted from the Poisson model because the creation of the free charge carriers (electrons and holes) are not independent events, but rather rely on the history of the recoil electron as it transfers its energy in the creation of additional electron-hole pairs and phonon excitations [39]. As a result, the variation in the number of charge carriers is scaled by the Fano factor that corrects the Poisson model for the number of the observed charge pairs [40]. The Fano factor is defined as

$$
F=\frac{\sigma(E)^{2}}{N(E)} .
$$

Measured values for the Fano factor vary from 0.084 to 0.16 in silicon [6]. For this study a Fano factor equal to 0.1 is used. The mean number of charge pairs as a 


\begin{tabular}{ccccccc}
$\begin{array}{c}E_{\text {recoil }} \\
(\mathrm{keV})\end{array}$ & $N_{\text {pairs }}$ & $\sigma_{\text {pairs }}$ & $\begin{array}{c}\mathrm{I}(\mathrm{nA}) \\
(\tau=200 \mathrm{~ns})\end{array}$ & $\begin{array}{c}\mathrm{I}(\mathrm{nA}) \\
(\tau=30 \mathrm{~ns})\end{array}$ & $\begin{array}{c}\Delta \mathrm{I}(\mathrm{pA}) \\
(\tau=200 \mathrm{~ns})\end{array}$ & $\begin{array}{c}\Delta \mathrm{I}(\mathrm{pA}) \\
(\tau=30 \mathrm{~ns})\end{array}$ \\
\hline 30 & 8300 & 29 & 13 & 89 & 46 & 309 \\
100 & 28000 & 53 & 44 & 298 & 85 & 565 \\
200 & 56000 & 75 & 90 & 597 & 120 & 800 \\
340 & 94000 & 97 & 150 & 1003 & 155 & 1035 \\
\hline
\end{tabular}

Table 3.1: $N_{\text {pairs }}=E_{\text {recoil }} / \eta$ where $\eta$ is the energy required to create an electron-hole pair, I (nA) are rough estimates of the current assuming collection times $\tau$ of 200 ns (close to the $p^{+}$side) or $30 \mathrm{~ns}$ (close to the $n^{+}$side), and $\Delta \mathrm{I}(\mathrm{pA})$ is the average variation of the current due to the uncertainty in the number of charge carriers $\sigma_{\text {pairs }}$.

function of initial recoil electron energy is shown in Table 3.1 along with the standard deviation $\sigma$ found by solving Eq. 3.11 for $\sigma$. The threshold voltage used in this study is $30 \mathrm{keV}$, and an energy of $340 \mathrm{keV}$ corresponds to the Compton Edge of $511 \mathrm{keV}$ incident photons.

Another source of noise that degrades the energy resolution in silicon detectors is variations in the leakage current. There are two types of leakage current: bulk leakage current and surface leakage currents. The bulk leakage current arises due to the reverse bias voltage applied across the silicon detector thickness and the resistance $R$ of the silicon detector. If ohmic contacts are used to collect the free charges then the leakage current (calculated simply using $I=V / R$ ) for our silicon detectors would be 0.6 A assuming $\rho=20 \mathrm{k} \Omega \mathrm{cm}$, an area of $10.0 \mathrm{~cm}^{2}$ and thickness of $1.0 \mathrm{~mm}$ to calculate $R$. Compared to the maximum average current from events occurring at the Compton edge (see Table 3.1) the average current is on the order of $1 \mu A$. For ohmic contacts, variations in the leakage current would completely dominate the energy resolution. For this reason, blocking contacts are used in the silicon detectors used in this experiment. Blocking contacts do not insert an electron or hole back into 
the silicon's active volume when an electron or hole is collected by the electrodes, thus reducing the leakage current from a fraction of an amp to a few hundred pico-amps. Variations in the leakage current at the pico-amp level can still degrade the energy resolution because these variations are of the same order as the uncertainty from the statistics of charge pair creation.

Surface leakage currents can arise due to impurities introduced, for example, by the atmospheric conditions that the detector was constructed and stored [33]. The silicon detectors used in this study use a guard ring connected to ground that significantly reduces the surface current contribution to the energy measurements. The total leakage current in the $1.0 \mathrm{~mm}$ thick silicon detectors used in this study was measured to be 100 pA using current-voltage studies performed by Studen [34].

Noise that degrades the energy resolution in the front-end electronics is due to a combination of current noise, voltage noise, and $1 / f$ noise. These three quantities are a function of the capacitance seen by the pre-amplifier, the detector leakage current, series resistance, and the temperature of the system [41] [42]. A detailed discussion of the noise due to the front-end electronics is not presented here because an accurate determination of the energy is not needed in this study.

\subsection{Our Silicon Detectors}

Two 512, $1.0 \mathrm{~mm}$ thick silicon pad detectors manufactured by SINTEF [43] are used in this experiment. Fig. 3.4 displays a cross section through a single silicon pad. Each silicon pad is $1.4 \mathrm{~mm} \times 1.4 \mathrm{~mm}$ arranged into a 32 x 16 array, with a total area of $44.8 \mathrm{~mm} \times 22.2 \mathrm{~mm}$. The perimeter of the silicon detector is surrounded by a guard ring connected to ground used to reduce surface leakage currents. The bulk 


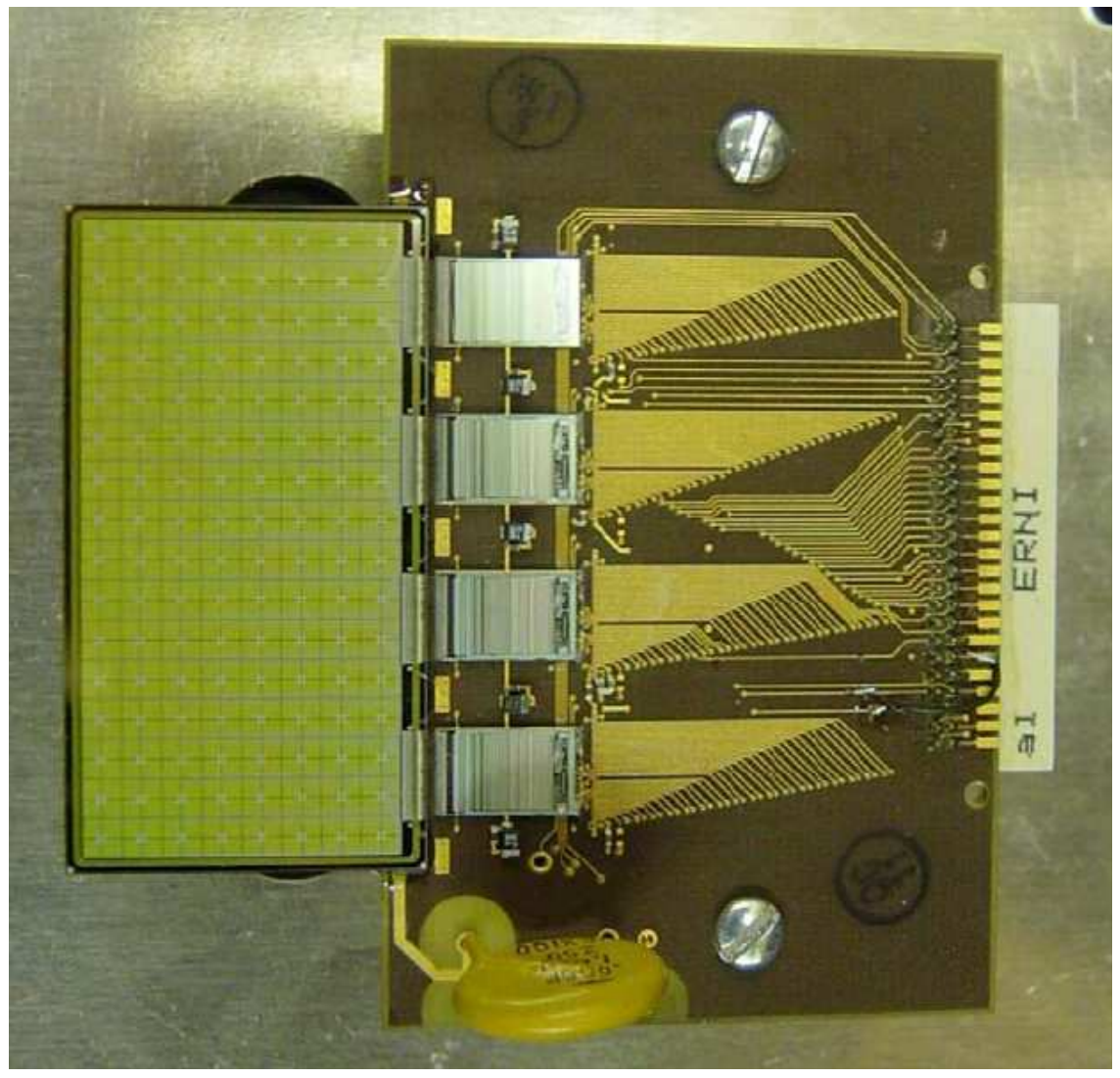

Figure 3.3: 512 pad $1.0 \mathrm{~mm}$ thick silicon detector. Each pad is $1.4 \mathrm{~mm} \mathrm{x} 1.4 \mathrm{~mm}$ in area. The detector is readout via four VATAGP3 ASICs that each readout 128 channels. 


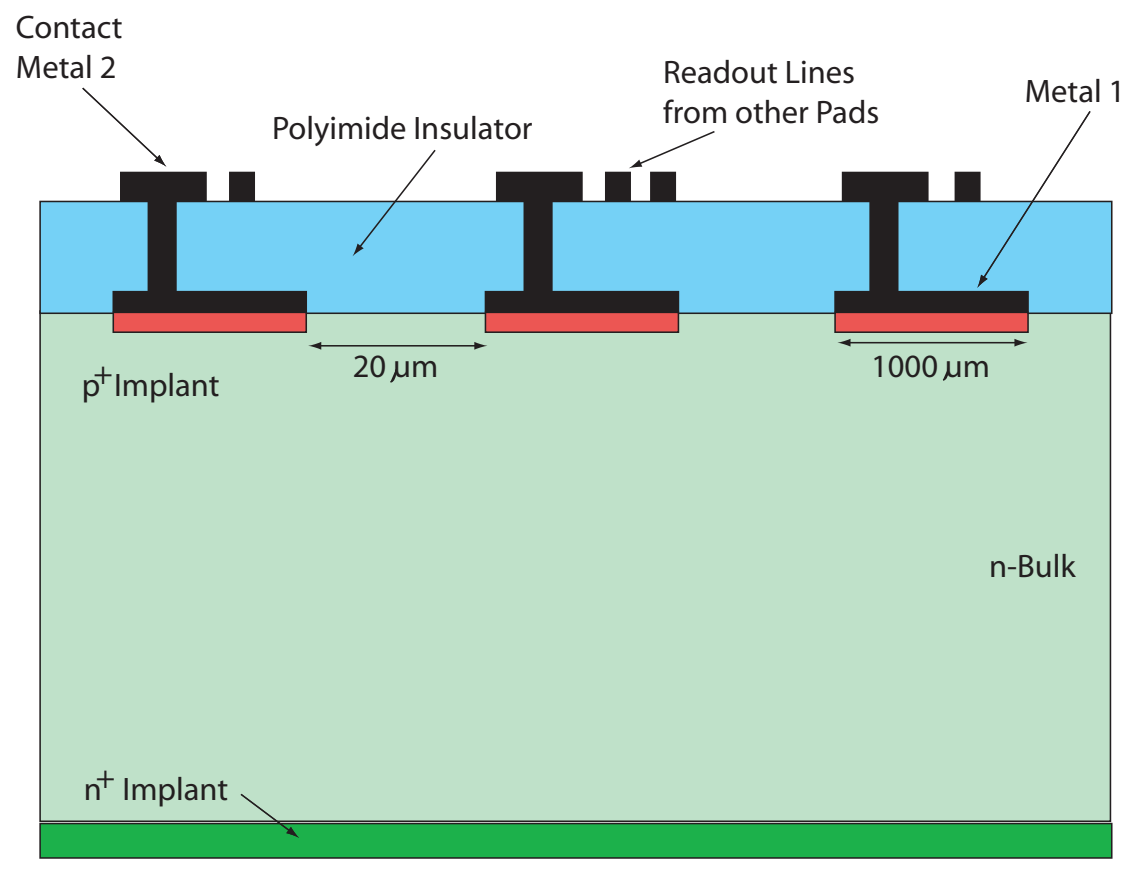

Figure 3.4: Cross section of a single silicon pad.

of the detector volume is composed of $n$-type silicon surrounded by heavily doped $p^{+}$ and $n^{+}$silicon on either edge of the detector thickness. There is a double metal layer on top of the $p^{+}$layer. The first metal layer is DC-coupled to the $p^{+}$layer. The first and second metal layer are separated by a thin $10 \mu \mathrm{m}$ layer of polymide insulation. The first metal layer is connected to the second metal layer by small $p^{+}$bias implants. The second metal layer routes the charge collected from the silicon to bonding pads on one side of the silicon detector. Each of the 512 pads on the edge of the detector are sequentially wire-bonded to separate channels on one of the four VATAGP3 chips. 


\subsubsection{VATAGP3 ASICS}

The role of the VATAGP3 chips are to determine if a physics event has occurred above the threshold voltage and to determine the amount of energy deposited in each silicon pad. The VATAPG3 chips interface with an intermediate electronics board that creates Nuclear Instrument Methods (NIM) level logic signals (logical true = $-0.8 \mathrm{~V}$, logical false $=0 \mathrm{~V}$ ) that are used to determine if the data collected by the VATAGP3 should be readout or discarded. If the data is to be readout, the VATAGP3 chips transfer the data to the intermediate board which then outputs the data to a local PC via a VME interface.

Figure 3.5 is a block diagram of the readout electronics for the first and last channel of a VATAGP3 chip. There are 128 identical readout channels arranged in parallel as indicated by the series of dots in the figure. The first stage of the VATAGP3 readout electronics, used to measure the energy of the pulse, consists of a charge sensitive pre-amplifier which amplifies and sums the charges arriving from a silicon pad. The output of the pre-amplifier is split into a fast shaper that determines if the voltage pulse from the pre-amplifier exceeds the threshold voltage and to a slow shaper that performs a more accurate measurement of the energy deposited in a silicon pad. The following two paragraphs describe the fast and slow shaper, respectively.

The portion of the amplified pulse that is split to the fast shaper is used to quickly determine if an event is above the threshold voltage. This is accomplished through the use of a fast semi-gaussian shaper that is composed of a combination of a differentiator and an integrator (CR-RC shaper). The RC time constant is chosen such that the pulse rise is very quick, on the order of 200 ns. The output of the fast semi-gaussian shaper is shown in Fig. 3.6. The output of the fast shaper is fed to a level-sensitive 


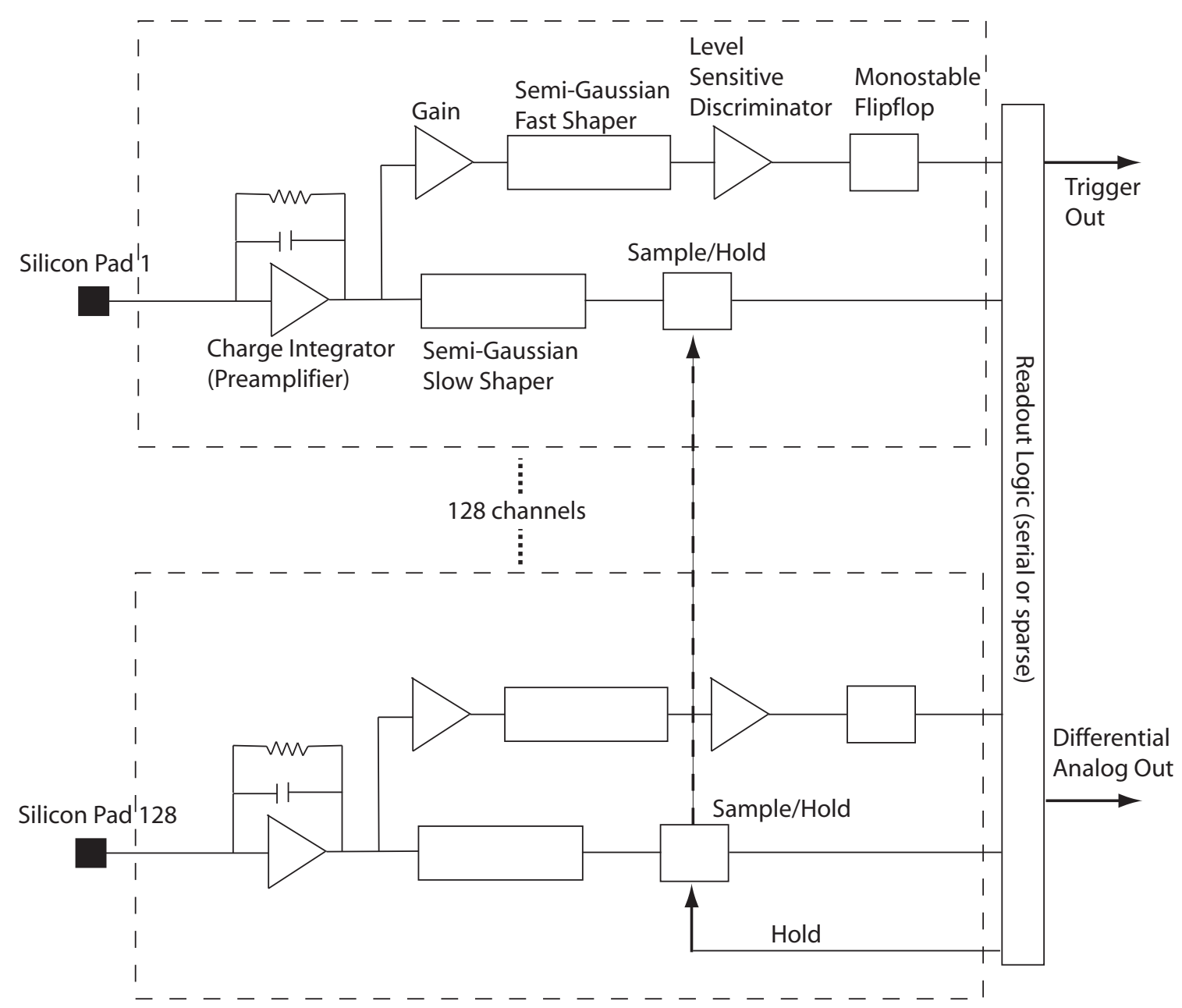

Figure 3.5: Example block schematic of the electronic circuits used to trigger and shape the signal for two silicon channel. There are 128 identical channels on each VATAGP3 chip. Image taken from [44]. A resistor was added in parallel to the capacitor in the charge sensitive preamplifier. This resister will discharge the capacitor overtime with a characteristic time much greater than the total charge collection time. This resister was mistakenly omitted in the previous publication. 
discriminator that compares the output voltage pulse from the fast shaper to a user specified threshold voltage. A trigger signal is produced if the fast shaper output voltage is greater than the discriminator threshold voltage.

An accurate measurement of the energy deposited in a silicon pad is determined by a slow semi-gaussian shaper with a characteristic time $\tau$ much greater than the fast shaper such that all of the charge from the silicon detector can be collected. The output of the slow semi-gaussian shaper is shown in Fig. 3.6.

The full readout sequence as a function of time is shown in Fig. 3.6. The readout cycle is initiated by a photon interaction in the silicon that gives rise to a small current pulse. The charge sensitive pre-amplifier converts and amplifies the charge into a voltage pulse that is split between a fast shaper, to determine if a trigger signal is to be produced, and slow shaper, to make a more accurate measurement of the energy deposited in the silicon. Lines three and four of Fig. 3.6 show the pulse shapes of the slow and fast shaper, respectively. As can be seen on line 4 , the voltage peak of the output from the fast shaper exceeds the threshold voltage of the discriminator (the dotted line) and a trigger pulse (TA) is produced. This trigger pulse causes a sample-and-hold ( $\mathrm{SH}$ ) trigger to go high after the $3 \mu s$ peaking time of the slow shaper. The SH stores the peak voltage of the slow shaper until the channel is readout or reset. Also at $3 \mu s$ the SHIFT-IN bit is set high, initiating a clock (CLK) that shifts from channel to channel reading the value stored in each channel's sample-and-hold buffer and sends each channel's stored analog value to the output (OUTP). Once the readout cycle is complete for all 128 channels on the chip, a SHIFT-OUT pulse is sent to the adjacent readout chip, initiating its readout cycle. 


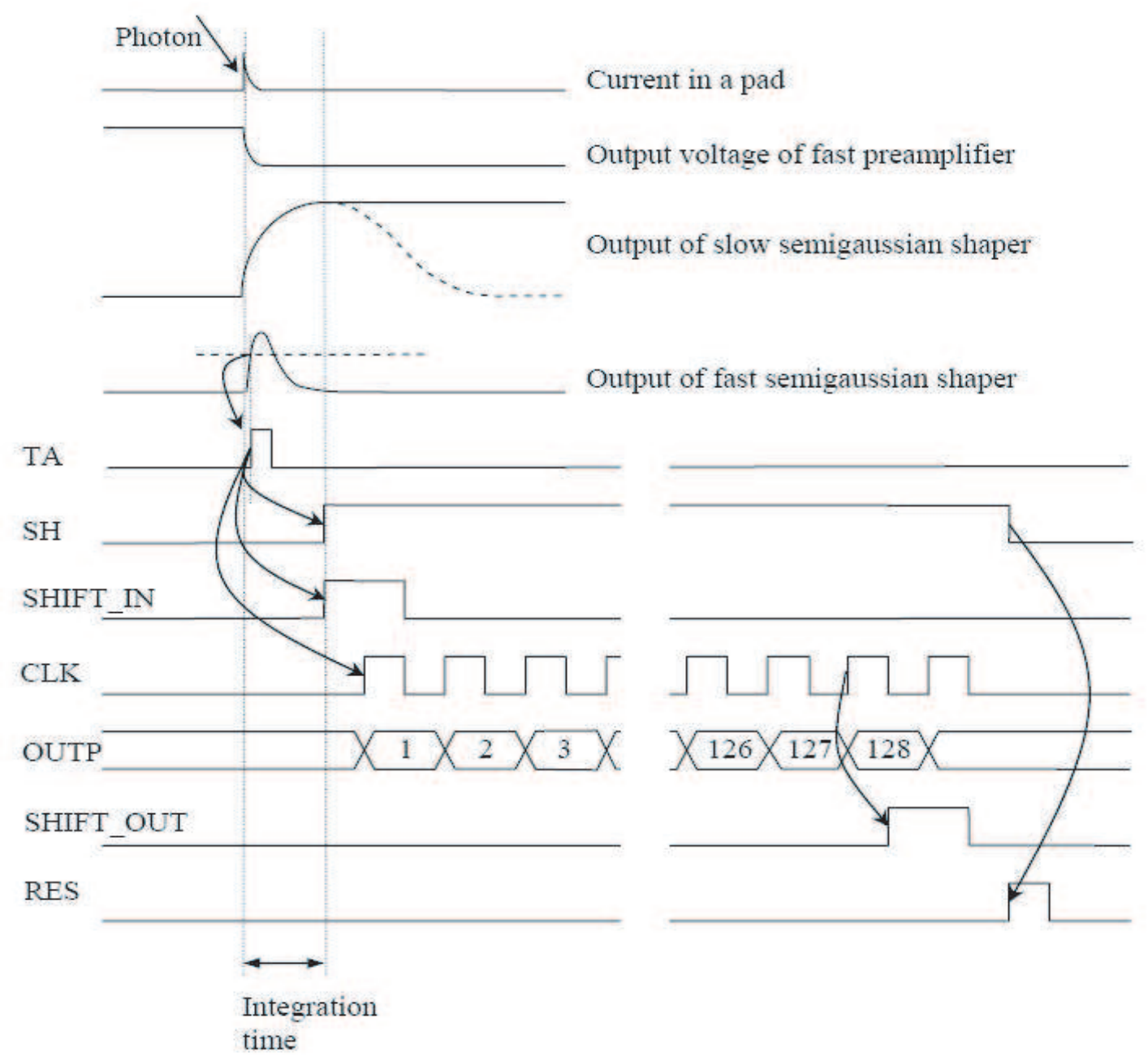

Figure 3.6: VATAGP3 readout cycle for an example silicon event. Image taken from [44]. 
At the end of the current chip's readout cycle the SH goes low, causing a reset (RES) signal to clear the system for the next event.

The analog output pulse (OUTP) is readout through an intermediate electronics board that communicates with the VME (Versa Module Europa) readout module. The output pulse from the intermediate board is digitized by analog-to-digital converters (ADCs) on the VME board. The digital signals from each chip are then stored in a compressed data file on the PC for analysis.

\subsubsection{Performance}

The format of the silicon data is a list of integers (128 integers for each chip) representing the total charge collected by each of the pads for a single event. The task is to determine the energy recorded in each pad on an event by event basis, and then determine which pads are above the threshold energy. For the small animal PET prototype described in this document, only the position information is used to reconstruct the images. The energy is used only to determine in which pad the event occurred; therefore, an accurate estimate of the energy is not essential for this experiment.

For other related experiments, such as the Compton Camera and Compton PET, similar silicon detectors are used in which the energy resolution is crucial for ideal system performance [35] [36]. For both cited applications the desired energy resolution of the silicon detectors is below $1.0 \mathrm{keV}$ (our detectors have energy resolutions around $1.5 \mathrm{keV}$ ) in order to reduce the uncertainty of the back-projected Compton scattering cones during the reconstruction process [45]. The following analysis was developed to measure the true energy resolution of silicon detectors that will be used in other 
imaging modalities. The energy resolution results for the silicon detectors used in this experiment are presented here for completeness.

This section outlines the steps of the energy resolution calculation for a standard ${ }^{241} \mathrm{Am}$ calibration point source. ${ }^{241} \mathrm{Am}$ is a radioactive element that emits two alpha particles (with energies of $5.44 \mathrm{MeV}$ and $5.49 \mathrm{MeV}$ ) and two gamma rays (with energies of $59.5 \mathrm{keV}$ and $2.6 \mathrm{keV}$ ). The ${ }^{241} \mathrm{Am}$ source is placed on top of an enclosed plastic box that contains a silicon detector. The detector is positioned approximately one centimeter below the top of the box. The alpha particles are completely absorbed by the plastic box and never reach the silicon detector; however, the gamma rays will penetrate through the plastic and interact with the silicon. The $2.6 \mathrm{keV}$ gamma ray is not recorded because it is below the $30 \mathrm{keV}$ minimum threshold of the detectors used in this study. The $59.5 \mathrm{keV}$ gamma ray is absorbed by the silicon, and the resulting photo-absorption peak is used to measure the energy resolution of the detectors.

Two separate silicon detectors are analyzed in this section and are labeled as OSU1 and OSU2. It must be noted that noisy channels and dead channels are not included in the energy resolution analysis. This analysis gives the best possible total energy resolutions for the two silicon detectors. With the exeption of the masked channels (1-64) on OSU2, all channels masked in this calibration study are also masked in full small animal PET set-up. Channels 1-64 on OSU2 are only masked for this resolution study, and are unmasked for the small animal PET coincidence data. The reason for this is that during the construction process of the small animal PET test-bench approximately half of the wirebonds connecting the VATAGP3 chip 1 to the silicon detector were damaged and needed to be replaced. After rewirebonding the damaged channels, the common mode noise dominated the image resolution, but only if these 
channels are left unmasked (the FWHM resolution was degraded by approximately 25 percent). For this reason these channels are masked to demonstrating the best possible operation of the OSU2 detector. Unmasking these channels during the final data collection does not degrade the final images because an accurate estimate of the energy is not needed in this setup.

\section{Pedestal Calculation}

As described in section 3.4.1, each channel has an average base-line voltage off-set (refereed to as a pedestal) determined internally by the VATAGP3 chips. In order to determine the pedestal for each channel, the mean of the variation of the base-line voltage off-set must be calculated. In the absence of a physics event, the voltage off-set for each channel will vary about a mean value due to the electronic noise of the system.

Plots of the pedestals versus channel number for the two silicon detectors used in this experiment are shown in figure 3.7. The pedestals were calculated using 4000 events. The first 2000 events were used to make a rough estimate of the pedestals that include contributions from physics events by simply taking the mean of the data

for each channel. After the rough pedestals have been calculated and a general idea of the pedestal value for each channel has been established, another 2000 events are used to calculate an improved estimate of the pedestals without the signal contribution. The sharp lines seen in Fig. 3.7 are broken (dead) channels. Figure 3.8 is a two dimensional representation of Fig. 3.7 taking into account the actual locations of the pads for OSU1 (left) and OSU2 (right). In the absence of a physics event and assuming no electronic noise the pedestals would not vary from event to event. 

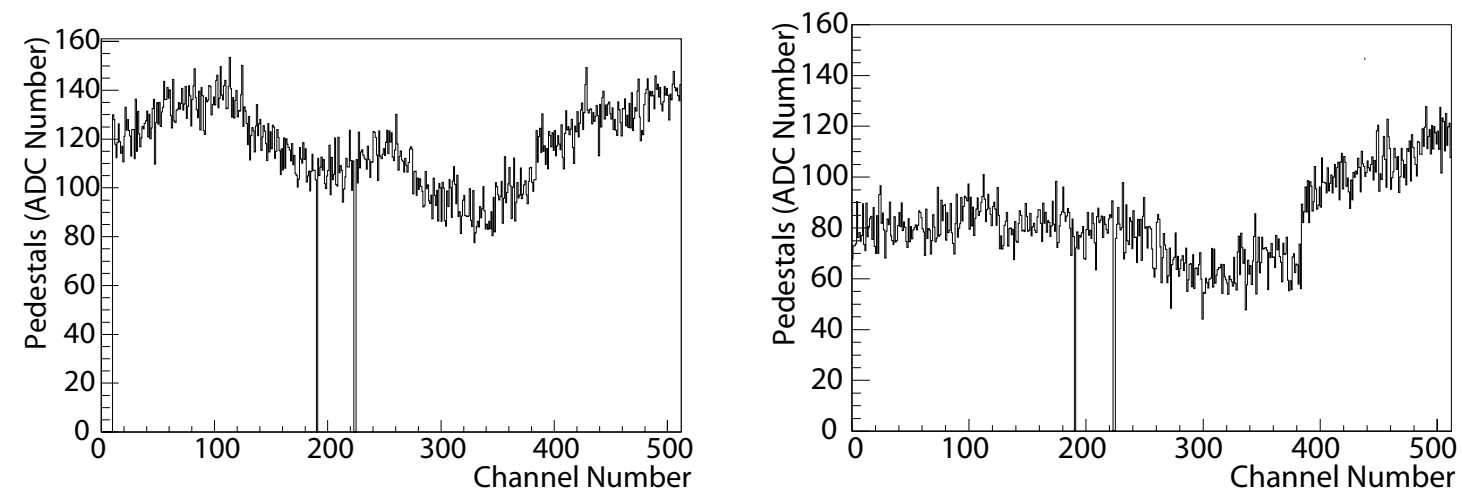

Figure 3.7: OSU1 (left) and OSU2 (right) pedestals versus channel number. Masked channels, OSU1: 1-10, 223,224 OSU2: 190,223,224
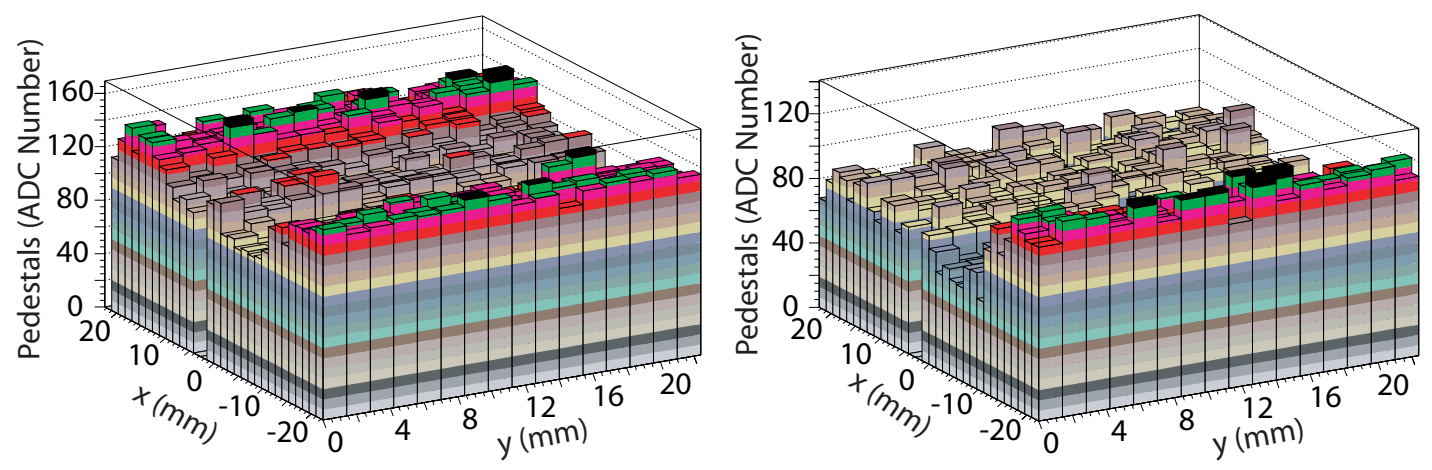

Figure 3.8: OSU1 and OSU2 pedestals at actual pad locations. Masked channels, OSU1: 1-10, 223, 224 OSU2: 190, 223, 224 


\section{Common Mode Noise Correction}

Common mode noise is electronic noise that is common to all channels and causes each channel to shift from its pedestal value by the same amount. Sources of common mode noise include electronic pickup from nearby electronic devices such as computers, power drills, printers, fluctuations in the power supply voltage, ground loops, etc ... [46]. Common mode noise in the current set-up varies from chip to chip; therefore, the correction looks for common voltage shifts on a chip-by-chip basis.

In order to calculate a common mode noise shift, the average noise for an entire chip must first be determined. After the final pedestal correction has been applied (event 4000), the next 20,000 events are used to calculate the total average noise for each chip (excluding channels above thresold of each event). After 20,000 events, the average noise of each chip is calculated for each subsequent event. The average event noise for each chip is compared to the previously calculated total chip noise average. The common mode noise is the difference between the average event noise and the total average noise. Fig. 3.9 and 3.10 show the common mode noise for the sample ${ }^{241} \mathrm{Am}$ calibration data for OSU1 and OSU2, respectively. The average FWHM for the OSU1 and OSU2 common mode noise is 1.3 and $1.2 \mathrm{ADC}$ channels or $0.40 \mathrm{keV}$ and $0.36 \mathrm{keV}$, respectively. 

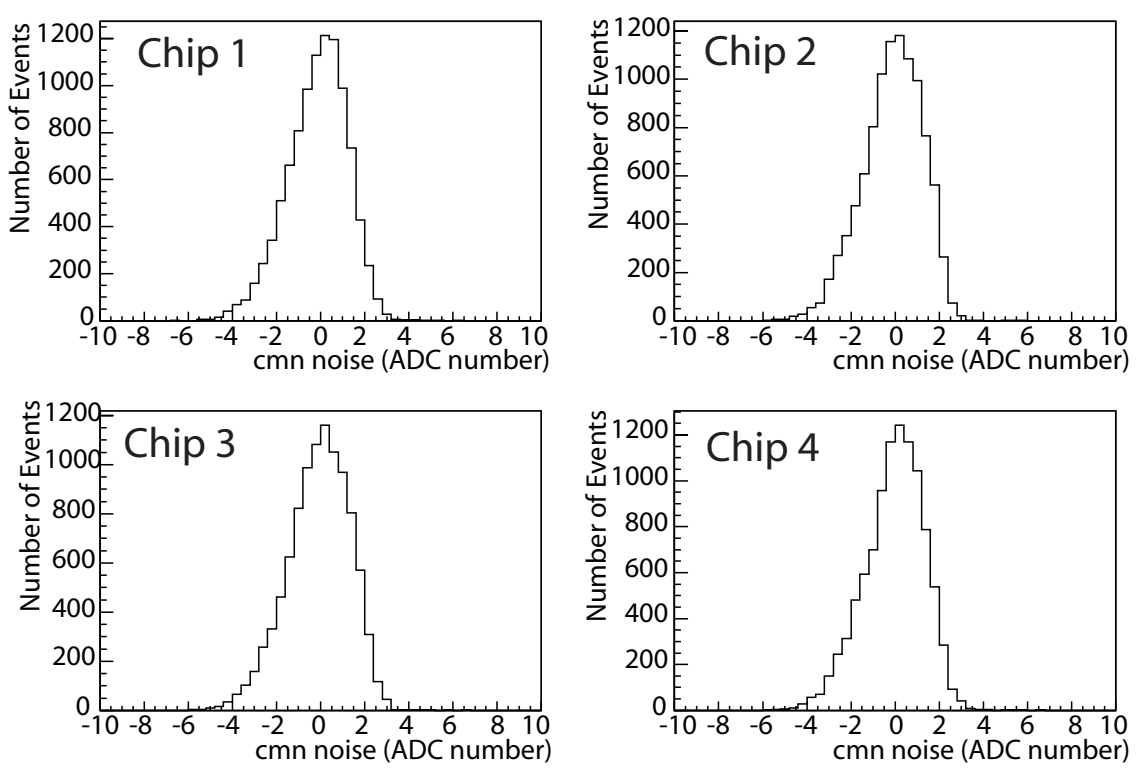

Figure 3.9: Histograms of OSU1 common mode noise per chip.
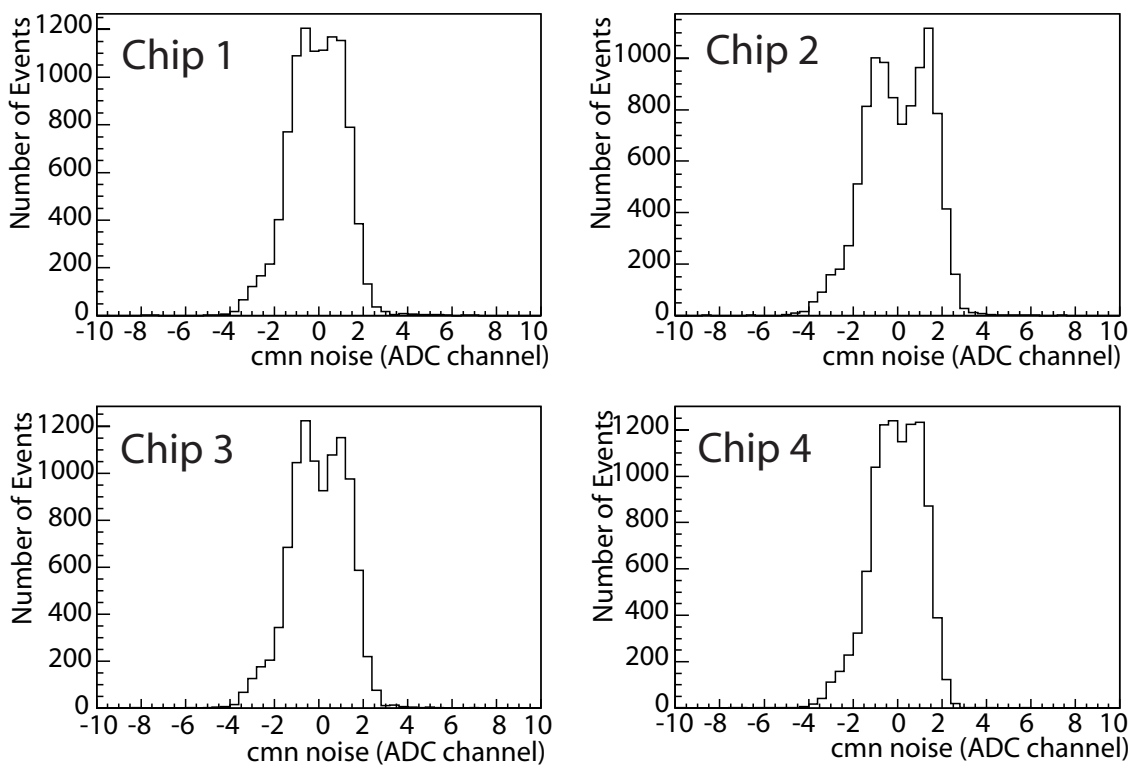

Figure 3.10: Histograms of OSU2 common mode noise per chip. As can be seen in the histograms there is a double peak that indicates the system is oscillating between two average noise values. The calculated average noise will fall between the two peaks. 


\section{Calculating the Gains}

Each channel will respond slightly differently to the same amount of collected charge causing the recorded energy spectrum to be shifted with respect to other channels. In order to correctly determine both the energy resolution of each channel and the total energy resolution of the detector, each channel must first be normalize such that each ADC bin represents the same amount of energy. This is accomplished by multiplying the bins of the each channel by a number $\alpha$ that shifts the energy peak of a calibration source into a user specified bin such that the mean of the calibration peak is centered about the same ADC value for every channel. This shift $\alpha$ is termed the gain, and varies from channel to channel.

The gain is calculated in this studying using an ${ }^{241} \mathrm{Am}$ point source positioned approximately a centimeter above the center of the detector. Before the gains can be calculated a pedestal and common mode noise corrected spectrum must be created for each channel. Fig. 3.11 is an example of a pedestal and common mode noise corrected channel for the OSU1 detector. The photo-absorption peak is fit to a Gaussian and the mean is extracted. The gain for the channel is determined by finding the value $\alpha$ that will shift the peak to the normalized channel. The ${ }^{241}$ Am peak is normalized to ADC channel 210 in this study because the ungain corrected data is distributed about this value (see Fig. 3.14 (Left)). The gain for each channel is calculated by first creating a pedestal and common mode noise corrected spectrum for each channel.

The gains versus channel number for both OSU1 and OSU2 are shown in Fig. 3.12. Two dimensional lego plots are shown in Fig. 3.13 of the gains versus actual channel number location for both detectors. Figures 3.14 and 3.15 are two dimensional histograms of the energy spectra for each of the 512 channels from OSU1 and OSU2, 

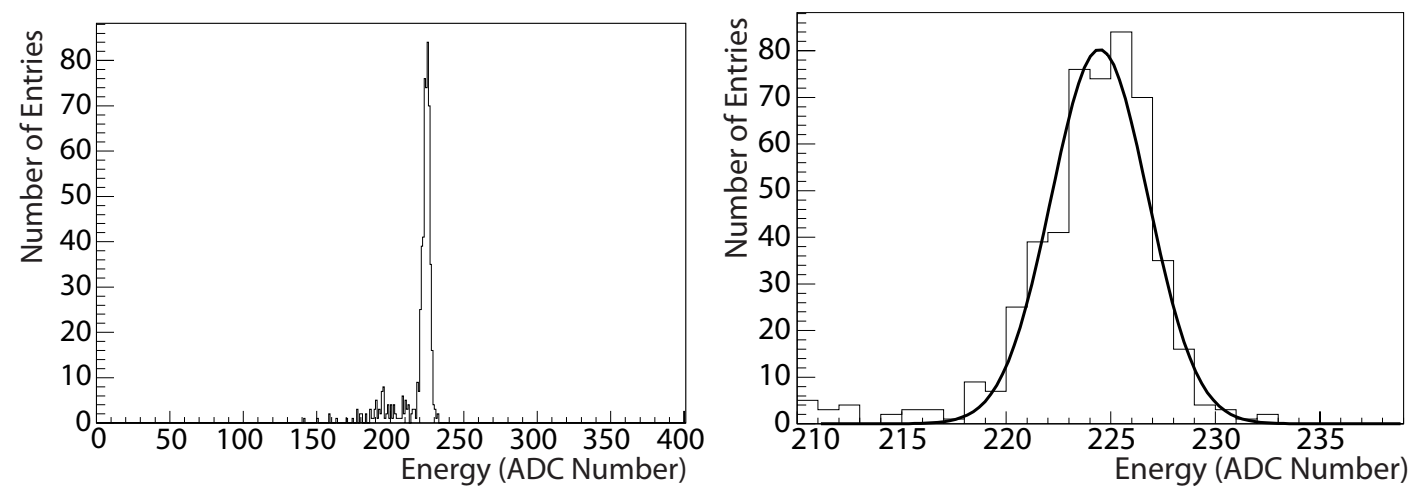

Figure 3.11: Example process for determining the gain of each channel. (Left) Single pedestal and common mode noise corrected spectrum from example channel 281 from OSU1. (Right) Zoomed in view of the photo-absorption peak and associated Gaussian fit. The mean is extracted from the Gaussian fit and is used to determine the gain.

respectively. The left image in these plots are of the pedestal and common mode noise corrected data. The images on the right are of the pedestal, common mode noise, and gain corrected data. It is clear from these images that the gain correction shifts the ${ }^{241} \mathrm{Am}$ photo-peak to channel 210 for every unmasked channel.
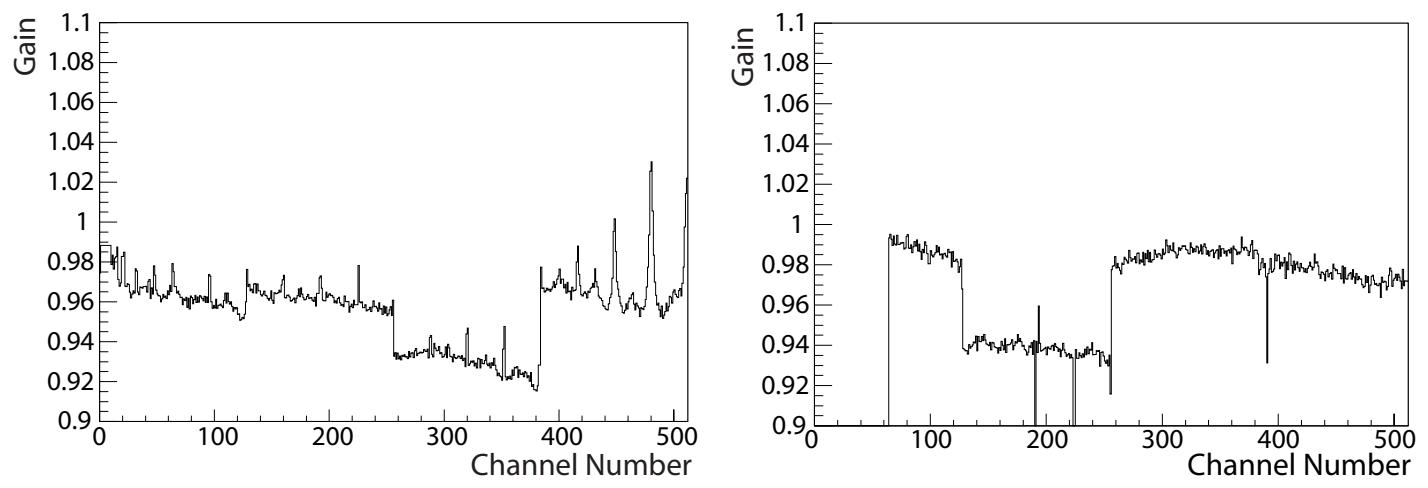

Figure 3.12: OSU1 and OSU2 gains versus channel number. Masked channels, OSU1: 1-10, 223, 224 OSU2: 1-64, 190, 223, 224 

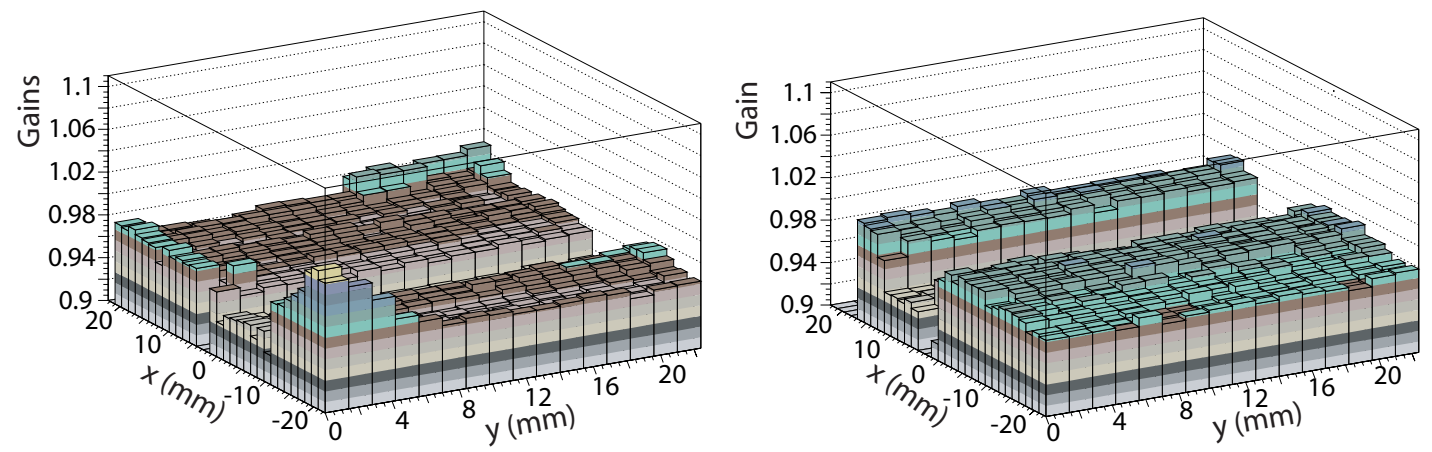

Figure 3.13: OSU1 and OSU2 gains versus channel number. Masked channels, OSU1: 1-10, 223, 224 OSU2: 1-64, 190, 223, 224
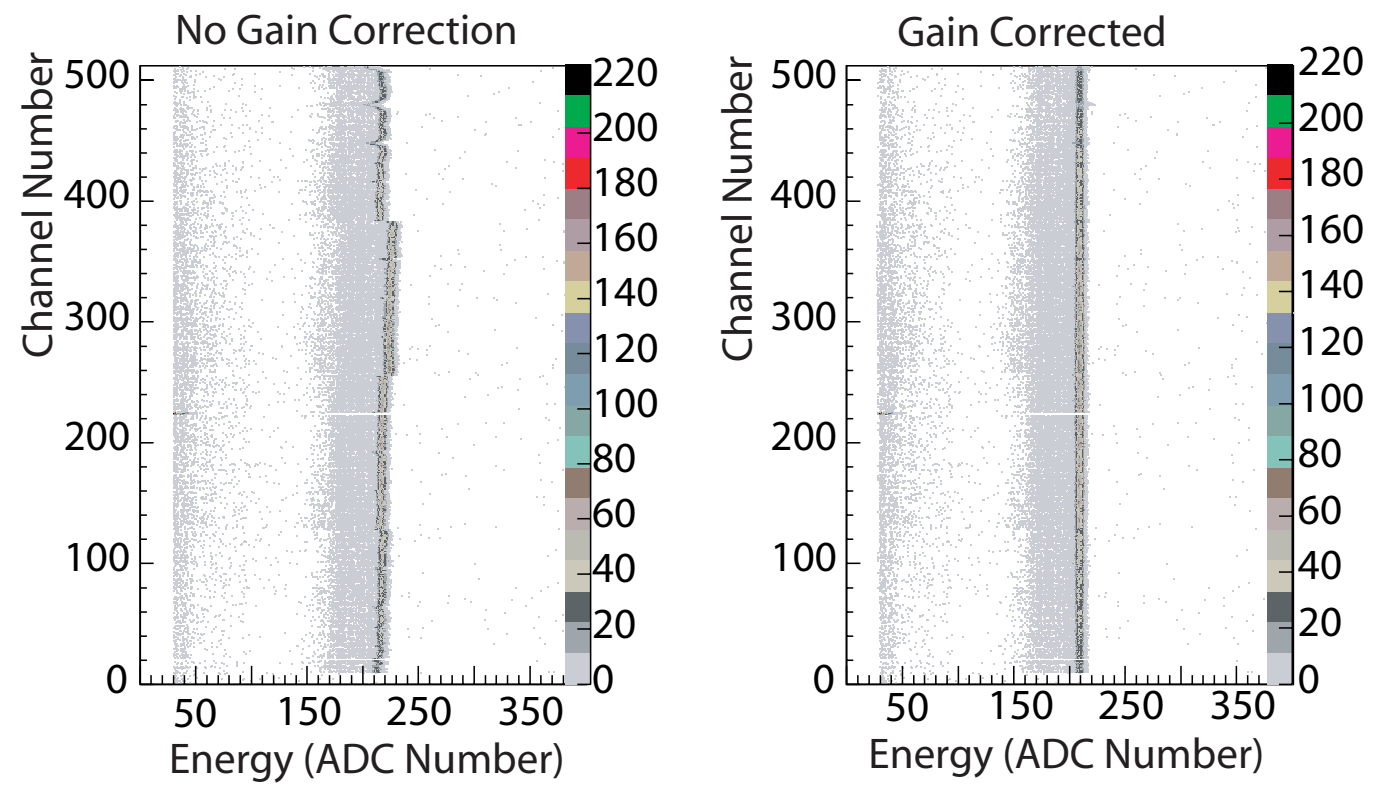

Figure 3.14: OSU1 pedestal and common mode noise corrected ${ }^{241} \mathrm{Am}$ spectrums for each channel (Left) without a gain correction and (Right) with a gain correction. Masked channels, OSU1: 1-10, 223, 224 OSU2: 1-64, 190, 223, 224 

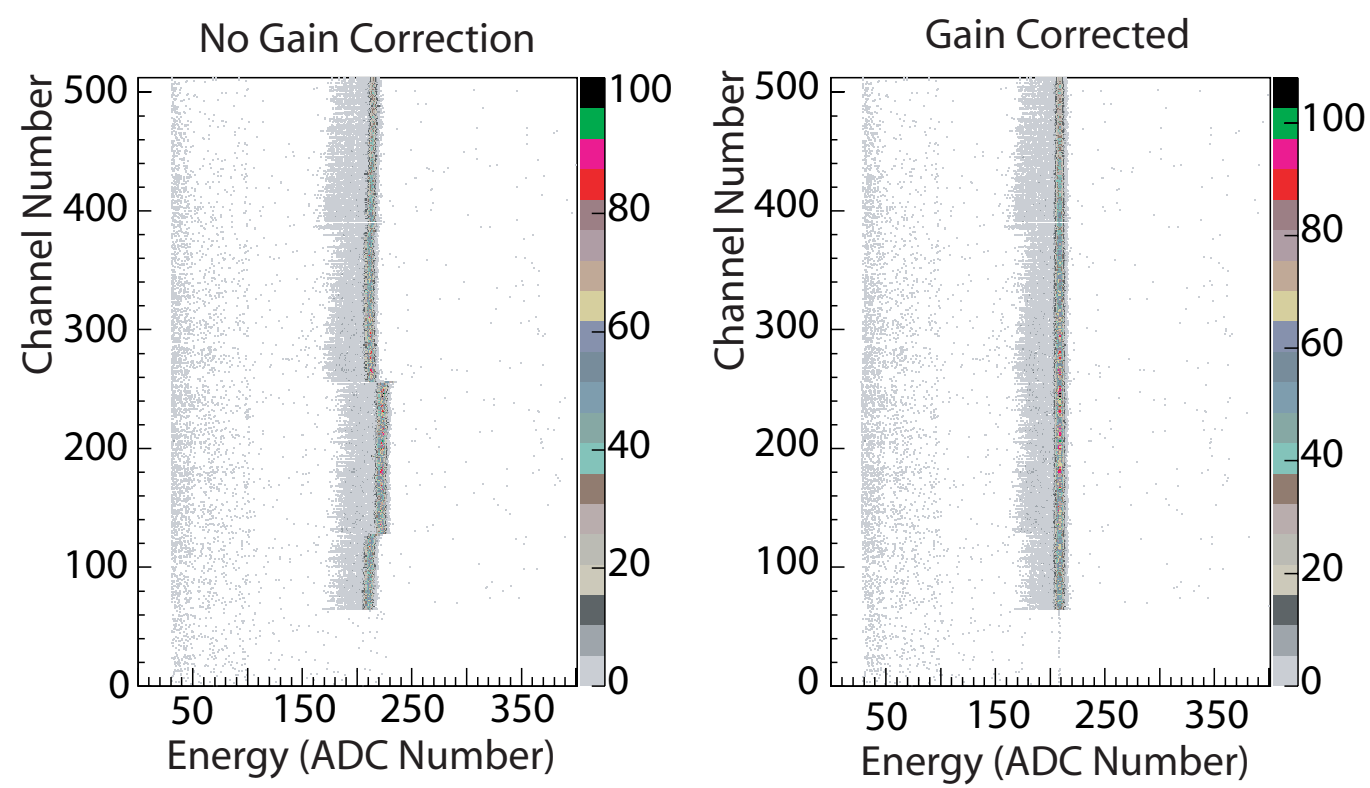

Figure 3.15: OSU2 pedestal and common mode corrected ${ }^{241} \mathrm{Am}$ spectrums for each channel (Left) without a gain correction and (Right) with a gain correction. Masked channels, OSU1: 1-10, 223, 224 OSU2: 1-64, 190, 223, 224

\section{Detector Resolutions}

The resolution of each channel for both OSU1 and OSU2 is plotted in Fig. 3.16. Figure 3.17 is the $2 \mathrm{D}$ equivalent with the channel numbers distributed according to their actual, physical positions. The resolution of each channel is calculated by first fitting the gain corrected spectrums for each channel with a Gaussian and then extracting the standard deviation $\sigma$ from each fit. The FWHM of each channel is found using

$$
F H W M=2.35 \sigma \frac{E_{c a l}}{\mu_{A D C}}
$$

where the quotient converts $\sigma$ from an ADC channel numbers to an energy measured in $\mathrm{keV} . E_{c a l}$ is the energy of the calibration peak $\left(59.5 \mathrm{keV}\right.$ for $\left.{ }^{241} \mathrm{Am}\right)$ and $\mu_{A D C}$ is the mean of the peak in units of ADC channels (210 for this example). 

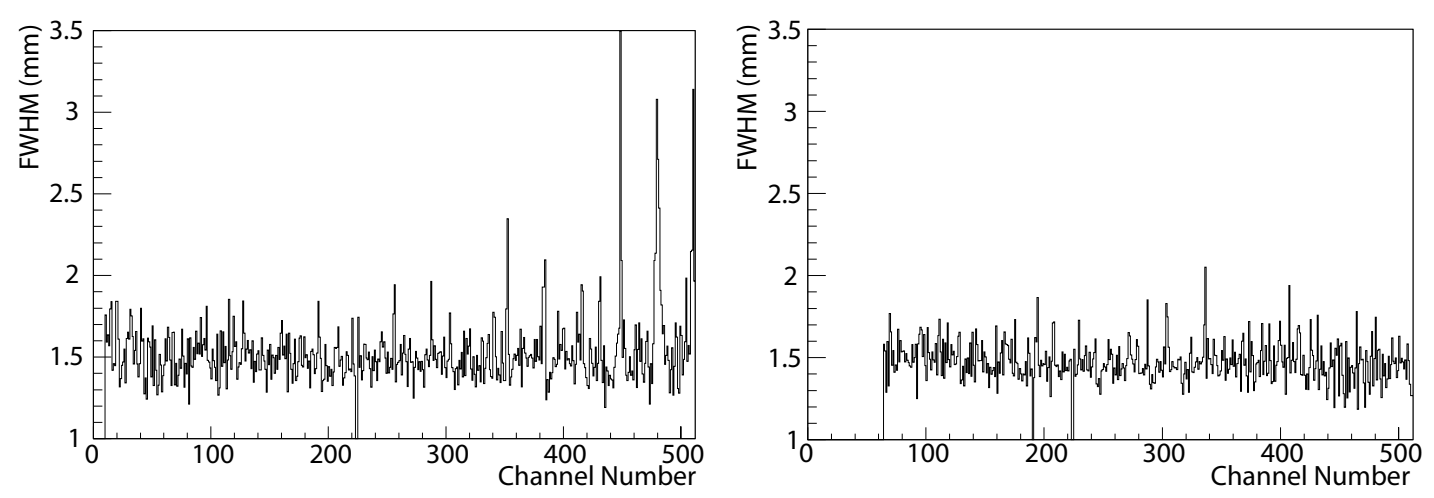

Figure 3.16: Sigma value of Gaussian resolution peak versus channel for OSU1 and OSU2, respectively.
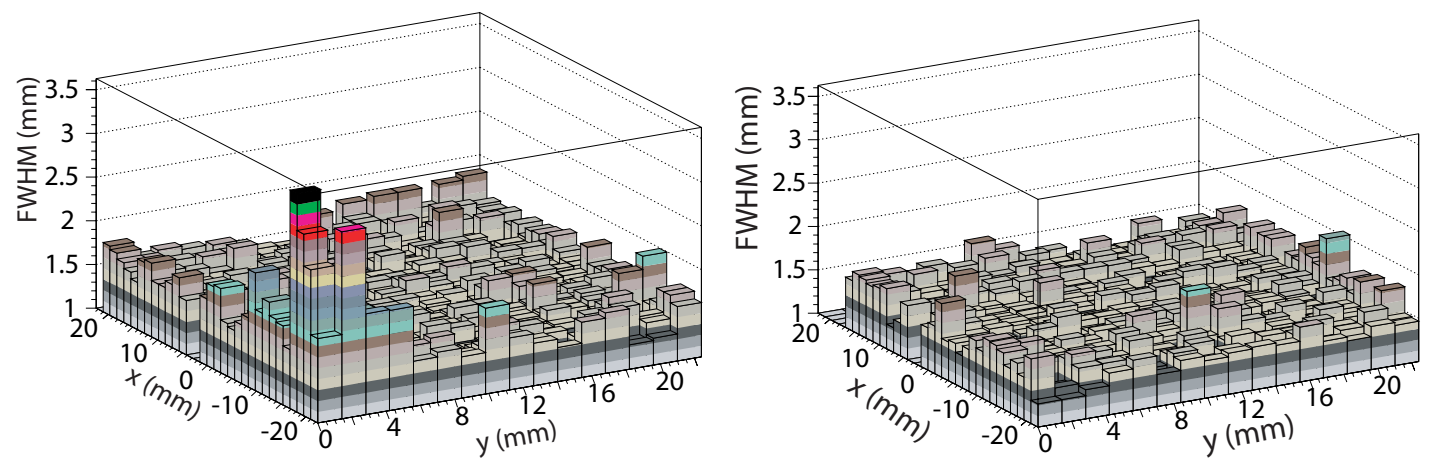

Figure 3.17: Sigma value of Gaussian resolution peak at the actual channel location for OSU1 and OSU2, respectively. 
The total resolution of the silicon detectors is defined to be the width of the peak at a particular energy when all of the channels are summed together. The total energy spectrum of an ${ }^{241} \mathrm{Am}$ calibration source is shown in Fig. 3.18 for OSU1 and OSU2, respectively. An artifically high threshold of $45 \mathrm{keV}$ was chosen in order to collect only events in the ${ }^{241} \mathrm{Am}$ photo-peak. The total resolution at $59.5 \mathrm{keV}$ of the OSU1 and OSU2 silicon detectors are $1.52 \mathrm{keV}$ and $1.50 \mathrm{keV}$, respectively.
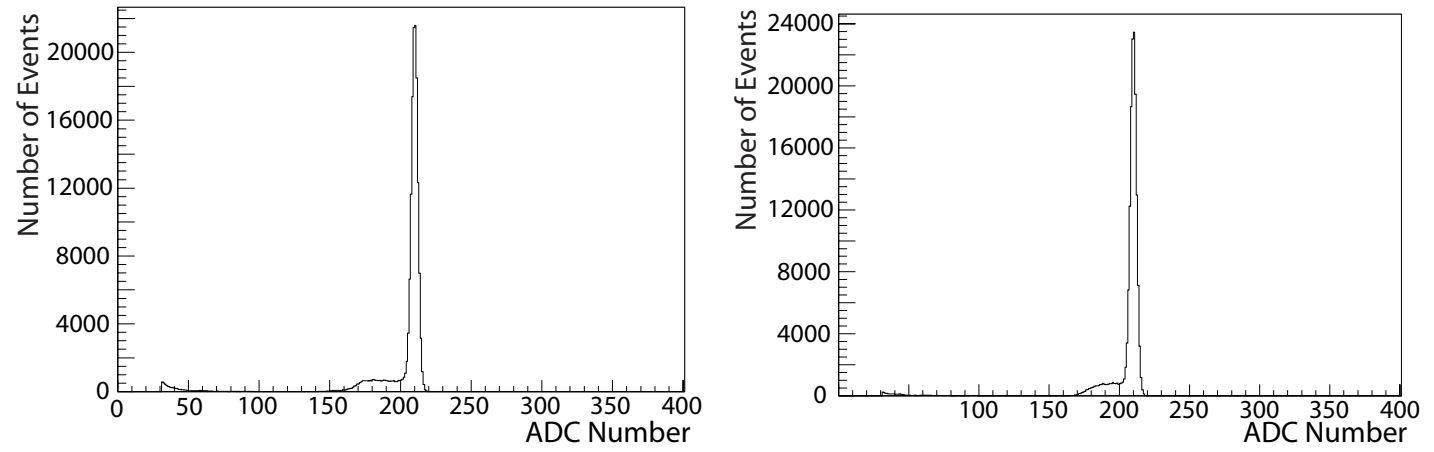

Figure 3.18: OSU1 and OSU2 pedestal, common mode noise, and gain corrected ${ }^{241} \mathrm{Am}$ total energy spectrums.

\section{Performance in Strong Magnetic Fields}

A silicon detectors and its associated front-end readout electronics were tested in the Ohio State University Medical School's 8 T large bore MRI magnet to determine if our silicon detectors could operate properly in the presence of a strong magnetic field. The silicon detector was left unmodified for the initial tests. After less than a minute of proper operation in a homogeneous $8 \mathrm{~T}$ magnetic field, the silicon detector stopped transmitting data due to a break in a few wire bonds between the hybrid and the VATAGP3 readout chips which supply power to the to the chips. 
It was hypothesized that the wirebonds broke due to the magnetic forces; however, the exact mechanism for the failure was unknown. There are two possible explanations. One, the force on the wirebonds was strong enough to pull the wirebonds off at the bonding point; however, this was unlikely because the wirebond feet were still attached. Two, the force deflected the wirebonds to the side as the current supplying power to the chip fluctuated causing a mechanical stress on the wirebonds over time, ultimately causing the wirebonds to break just above the feet.

In order to test this hypothesis an apparatus was constructed that consisted of a series of 1.0 mil and 1.25 mil diameter wire bonds that spanned a conduction path. Half of the wire bonds were encapsulated to test if the wire bonds would survive if denied the ability to move. The set-up consisted of a series of simple circuits that could be tested individually by adding or removing a jumper. Both direct current of varying magnitude and square wave alternating currents of varying frequency and amplitude were used in the test. A photograph of the device is shown in Fig. 3.19, and a schematic of a single wirebond circuit is shown in Fig. 3.20. Each wirebond circuit can be selected through the use of a jumper. The power supply can supply either DC or AC current. The wirebonds are connected in series with a $48 \Omega$ resister $R$.

The wirebonds were tested in two orthogonal orientations with respect to the magnetic field as shown in Fig. 3.21. The first magnetic field orientation in Fig. 3.21 (left) will cause a pulling and pushing force on the wirebonds depending on the direction of the current. The second orientation of the magnetic field will give rise to forces that will deflect the wirebond to the side. The third orthogonal magnetic 


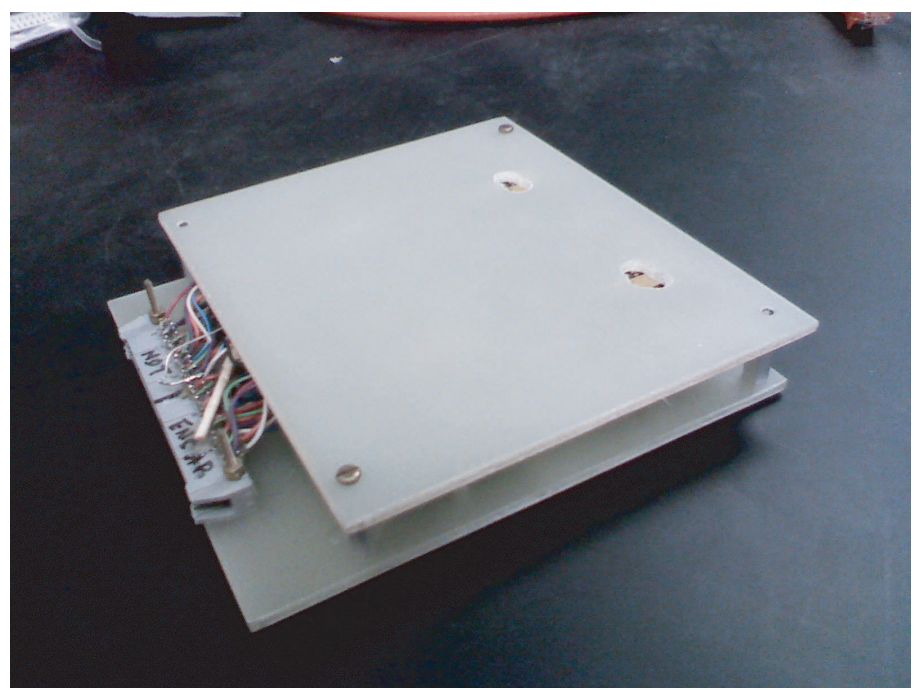

Figure 3.19: Photograph of the wirebond testing apparatus used to determine the reason why the wire bonds were breaking in the $8 \mathrm{~T}$ magnetic field, and to test if encapsulating the wirebonds could prevent the wirebonds from breaking. The holes drilled into the top of the device on the right are used to view the wirebonds.

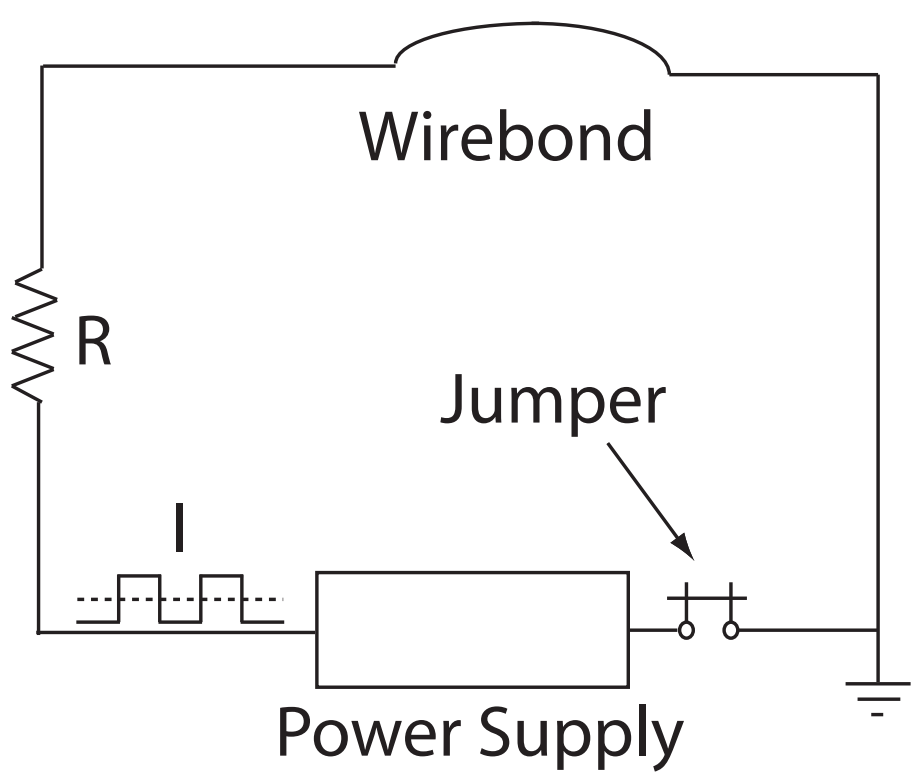

Figure 3.20: Schematic of a single wirebond circuit used to test the wirebonds in the 8 T magnet field. 
field orientation is in line with the current in the wirebond and will not give rise to a force.
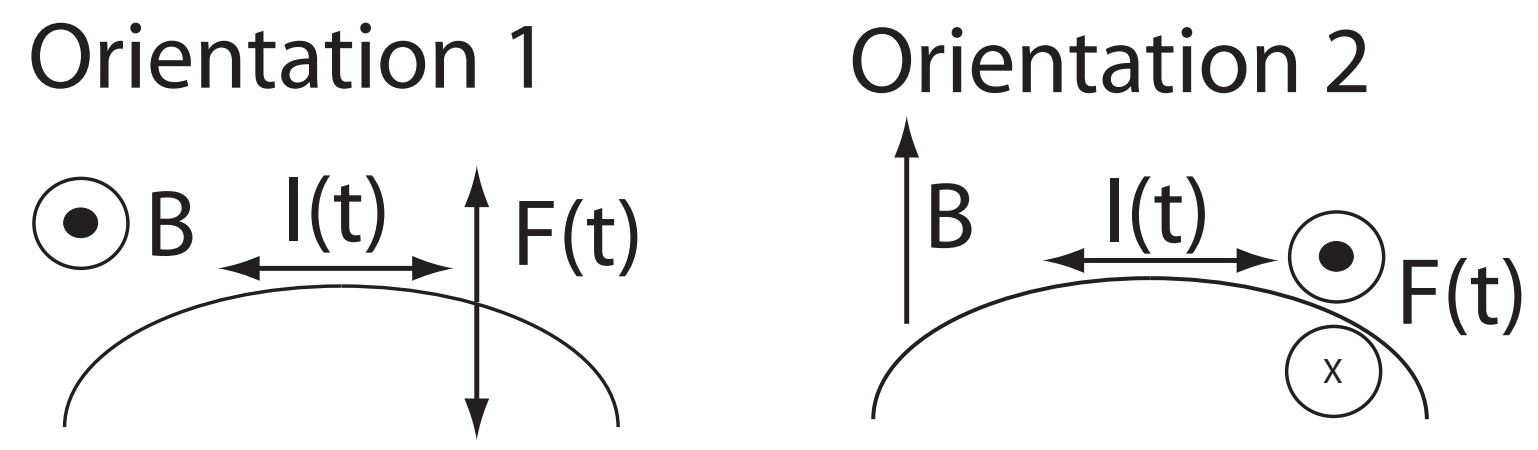

Figure 3.21: Image of the two magnetic field orientations tested on the wirebonds with alternating current $I(t)$ and the associated force on the wirebond $F(t)$. The magnetic field $B$ has a constant magnitude of $8 \mathrm{~T}$.

The force on the wirebonds due to a constant DC current, the least likely explanation for the broken wire bonds, was tested first in the $8 \mathrm{~T}$ field. For this test a constant DC current was produced by the power supply and fed into a 1.0 mil thick, unencapsulated, wirebond circuit. Both orientations relative to the magnetic field were tested. The current was ramped up from $0 \mathrm{mV}$ to $337 \mathrm{mV}$ (the maximum amount of current that could be generated by the power supply) in $25 \mathrm{mV}$ steps. The current was held stead for one minute at each voltage. Three identical wirebond circuits were tested in this manner. None of the wire bonds broke due to the magnetic force on a wire with a constant DC current.

A independent pull test was performed on a set of three 1.0 mil and three 1.25 mil wirebonds to determine how many grams of force would cause the wirebonds to dislodge at a foot. For the 1.0 mil and 1.25 mil wirebonds an average force of $9.6 \mathrm{~g}$ 
$(94 \mathrm{mN})$ and $12.36 \mathrm{~g}(121 \mathrm{mN})$ was required to pull the wirebond off at the foot. Using these forces $F$, the strength of the magnetic field $B$, and 1.0 millimeters for the wire length $L$ the maximum current required to break a wirebond in this manner can be calculate by the expression

$$
F=I B L
$$

To break the wirebonds by a pulling force would require $11.8 \mathrm{~A}$ and $15.1 \mathrm{~A}$ of current. The current flowing through the wire in the actual setup is on the order of $100 \mathrm{~mA}$, much too low to break the wirebonds in this manner. As expected, both of these tests confirm that the magnetic force due to a constant DC current did not break the wirebonds.

The next series of tests used square wave alternating currents in the two orientations shown in Fig. 3.21 for various frequencies. A current of $133 \mathrm{mV}$ maximum amplitude was chosen for this test in order to match the estimated variation in the current feeding power to the chips during a readout cycle. A frequency of $100 \mathrm{~Hz}$ was chosen because this is a typical readout frequency for our system. Five 1.0 mil unencapsulated wirebonds were tested. All five of the 1.0 mil wirebonds broke with an average time of 58 seconds. This is roughly the same amount of time it took for the original silicon detector to fail (approximately 1 minute). This test indicates that oscillations in the power line current on the order of $100 \mathrm{~mA}$ at a frequency on the order of $100 \mathrm{~Hz}$ causes mechanical stress on the wirebonds, eventually leading to a break just above a wirebond foot. Figure 3.22 shows an image of the broken wire bonds. 


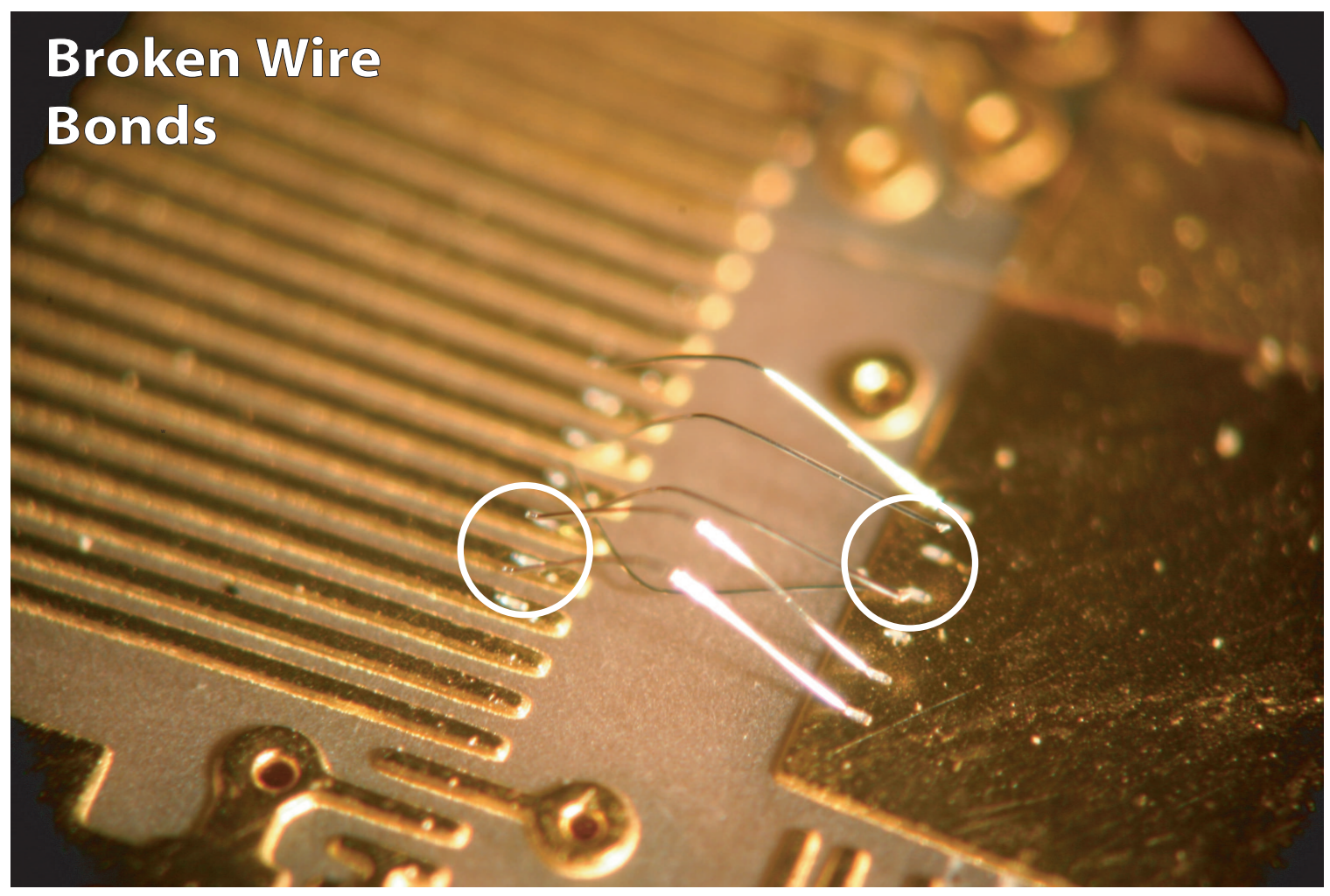

Figure 3.22: Image of wire bonds broken at the feet due to mechanical stress. 
The final wirebond study was used to test the encapsulated wirebonds under the same conditions that the unencapsulated wirebonds failed. Recall that the encapsulated wire bonds do not have the ability to oscillate. Two encapsulated wirebonds were each tested in the $8 \mathrm{~T}$ field with a AC square-wave current with amplitude 133 $\mathrm{mV}$ and a frequency of $100 \mathrm{~Hz}$ for 26 and 24 continuous hours, respectively. Neither wirebond broke. As a result of this study it was decided to encapsulate the wirebonds between the VATAGP3 chips and the silicon detector and test the full silicon detector setup again.

In order to test that the silicon detectors can not only collect data, but operate exactly as they do outside of the magnetic field, an ${ }^{241} \mathrm{Am}$ spectrum was collected at $8 \mathrm{~T}$ and compared to its $0 \mathrm{~T}$ counterpart. Figure 3.23 displays the energy spectrum of ${ }^{241} \mathrm{Am}$ at both $0 \mathrm{~T}$ and $8 \mathrm{~T}$. After correcting each channel of the silicon for the pedestals, common mode noise on a chip-by-chip basis, and applying a gain correction to each channel, the total energy resolution of the silicon detector was determined to be $1.6 \mathrm{keV}$ FWHM for both the $0 \mathrm{~T}$ and $8 \mathrm{~T}$ measurements. The energy resolution of the photo-absorption peak of the $59.5 \mathrm{keV}$ gamma ray emitted from the ${ }^{241} \mathrm{Am}$ source is identical within statistical variations at $0 \mathrm{~T}$ and $8 \mathrm{~T}$, thus demonstrating that silicon detectors can operate properly in magnetic fields up to $8 \mathrm{~T}$. 


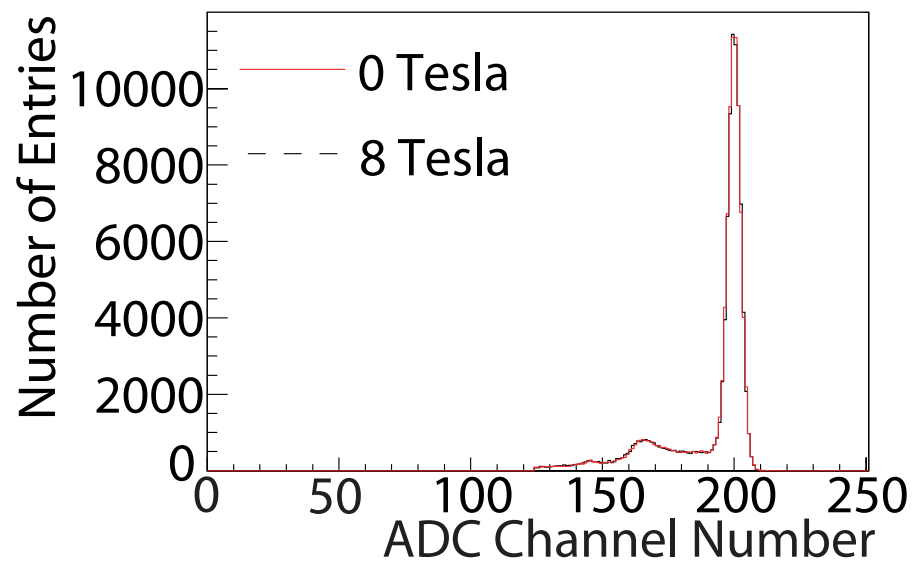

Figure 3.23: ${ }^{241} \mathrm{Am}$ energy spectrum at $0 \mathrm{~T}$ and $8 \mathrm{~T}$. The two distributions overlap almost perfectly. 


\section{CHAPTER 4}

\section{Experimental Setup}

\subsection{Experimental Setup Overview}

In order to measure the positron range in strong magnetic fields we had to design a very high resolution small animal PET prototype scanner using only non-magnetic components. Figure 4.1 is a diagram of the cross-section of the non-magnetic small animal PET apparatus, and Figure 4.2 is a photograph of the completed device. The mounting for the set-up is constructed using a combination of aluminum and acrylic plastic. Two $1.0 \mathrm{~mm}$ thick silicon pad detectors are positioned edgewise on opposite sides of the source. The silicon detectors are positioned on precision leveling mounts that are used to align the detectors. A laser beam is used to confirm that the silicon detectors are aligned into the $1.0 \mathrm{~mm}$ source plane. The silicon readout electronics, which have a few magnetic components, have been securely attached to the aluminum base-plate of the apparatus.

A clinical PET scanner is composed of a detection ring that completely surrounds the patient. Our prototype small animal PET has only two silicon detectors separated by approximately $17 \mathrm{~cm}$. In order to simulate a full detector ring and collect a data set with full angular sampling, data must be recorded at discrete angular intervals by 


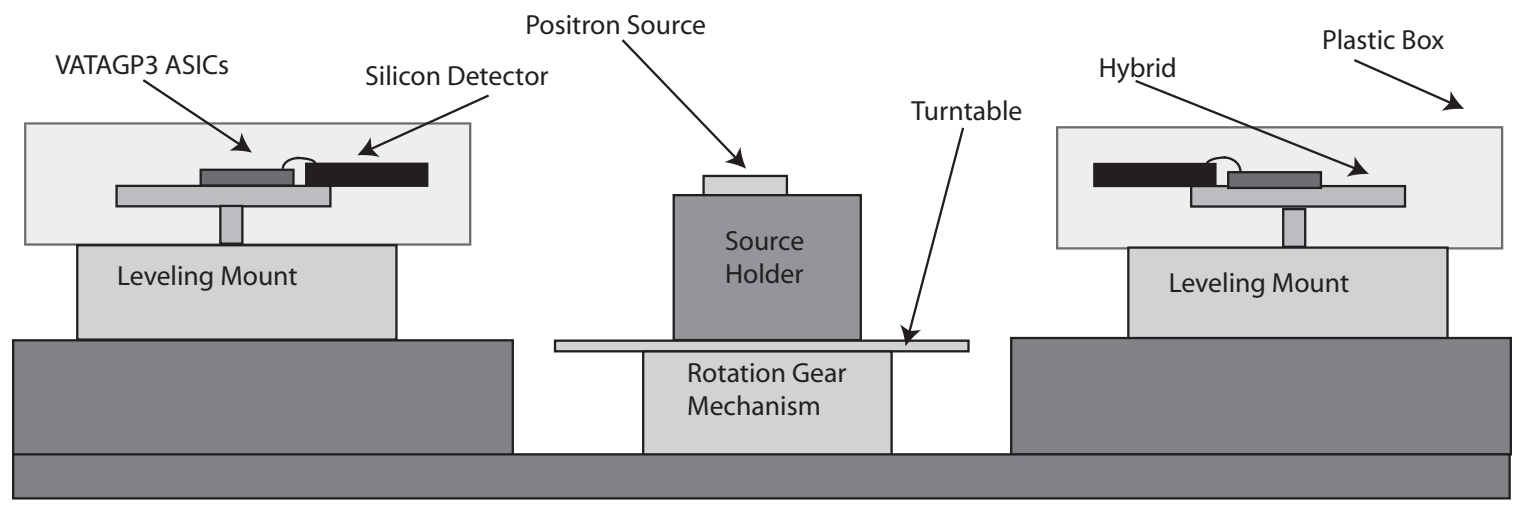

Figure 4.1: Cross-sectional diagram of the small animal PET test-bench.

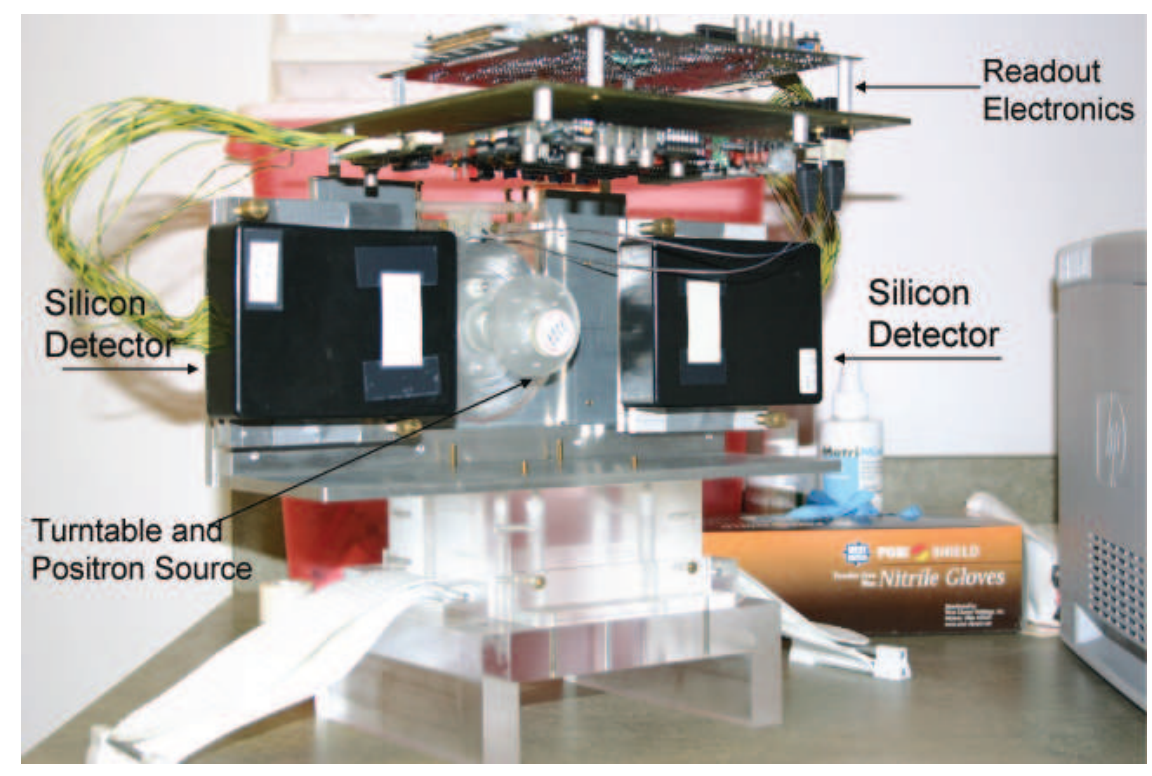

Figure 4.2: Photograph of the small animal PET test-bench as viewed from above relative to the cross-sectional diagram shown in Fig. 4.1. 
rotating the source distribution. This is analogous to rotating the detectors around a stationary source when the positron range distribution is symmetric (see Fig. 2.31). The source is secured to a turntable that can be rotated by a pneumatic pump in one degree increments.

\subsection{Rotation Mechanism}

In order to verify that the turntable is rotating properly, 180 holes were drilled into its the outer diameter and a pair of fiber optic cables were mounted in fixed positions above and below the outer diameter of the turntable (see Fig. 4.3). A beam of light is emitted through the upper fiber optic cable. When the turntable is rotated by one degree the light emitted through the upper fiber optic cable is either passed though one of the holes into the opposing cable or the light is blocked. The fiber optic light passed through the outer diameter of the turntable is converted into an electrical signal, such that the difference in voltage can be observed over a standard digital oscilloscope. In this way it is guaranteed that the source distribution is rotated properly.

\subsection{Choosing a Coincidence Timing Window}

The coincidence timing window is the amount of time the system waits for a trigger signal from the opposing silicon detector before sending a reset signal back to the detector that registered an event above the threshold voltage. The coincidence timing window should be chosen with care. The larger the coincidence timing window the more random events will be recorded, thus degrading the final image resolution. On the other hand, if the coincidence window is too small, actual coincidence events 


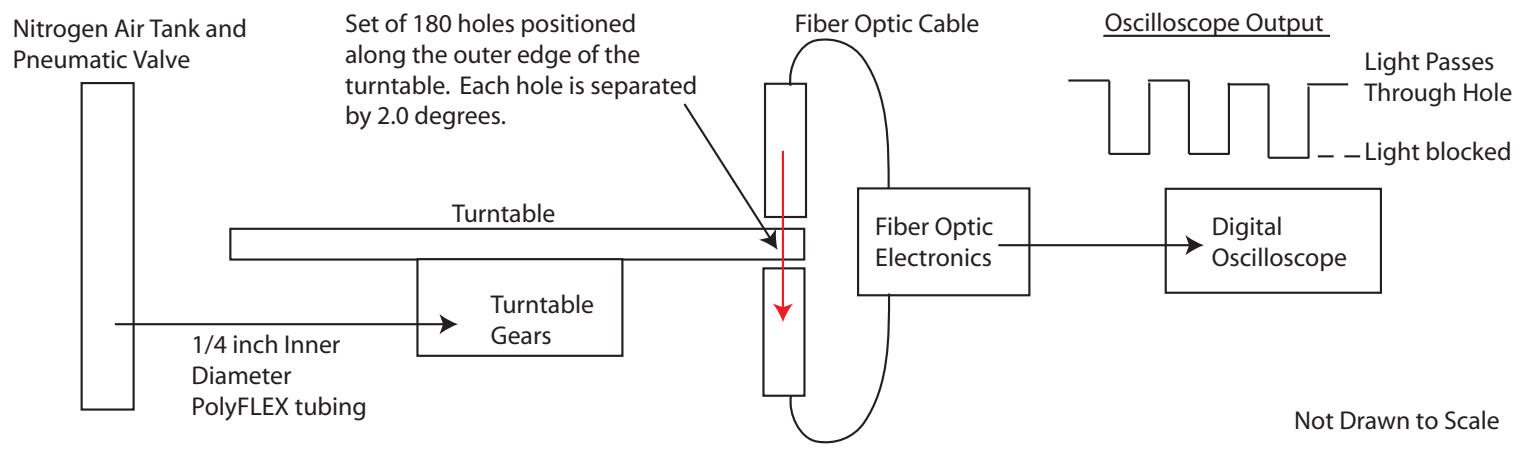

Figure 4.3: Block diagram of source rotation mechanism used to collect data with full angular sampling. The fiber optic mount is use to confirm that the source is properly rotated.

may go undetected, resulting in a system with lower sensitivity. Clinical PET scanners have coincidence timing windows on the order of a few nanoseconds to hundreds of picosecond [2]. This short timing window is possible due to the quick response times of the photon detectors and their associated readout electrons. The short timing window is also necessary for reducing random coincidence that become more common with higher activity sources.

The setup shown in Fig. 4.4 was used to measure the coincidence timing uncertainty for our system. The coincidence timing measurement consisted of a collimated ${ }^{18} \mathrm{~F}$ line source position in the center of the FOV between the two silicon detectors, OSU1 and OSU2. The output triggers from OSU1 were sent directly to the START input of an ORTEC 566 time-to-digital converter (TDC), while the output triggers from OSU2 were delayed by 384 ns before being sent to the STOP input of the TDC. The TDC measures the time difference between the two pulses and outputs the result. The second pulse is delayed so that both sides of the coincidence curve can 


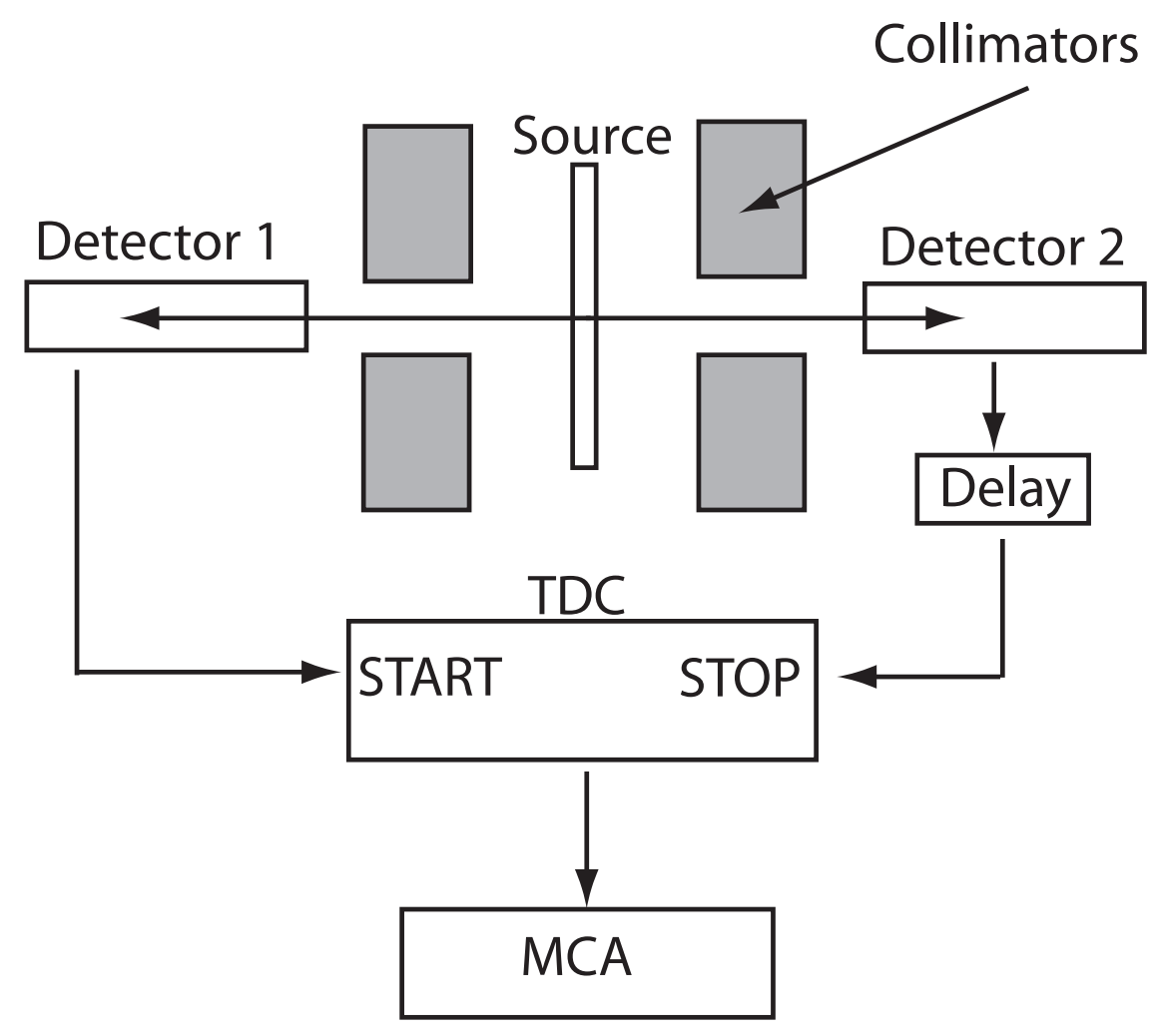

Figure 4.4: Coincidence measurement setup consisting of a collimated ${ }^{18} \mathrm{~F}$ line source position in the center of the FOV of the two silicon detectors. The output triggers from OSU1 were sent directly to the START input of a time to digital converter (TDC), while the output triggers from OSU2 were delayed by 384 ns before being sent to the STOP input of the TDC. The TDC output (the time difference between the two trigger signals) is digitized by a multi-channel analyzer (MCA). 


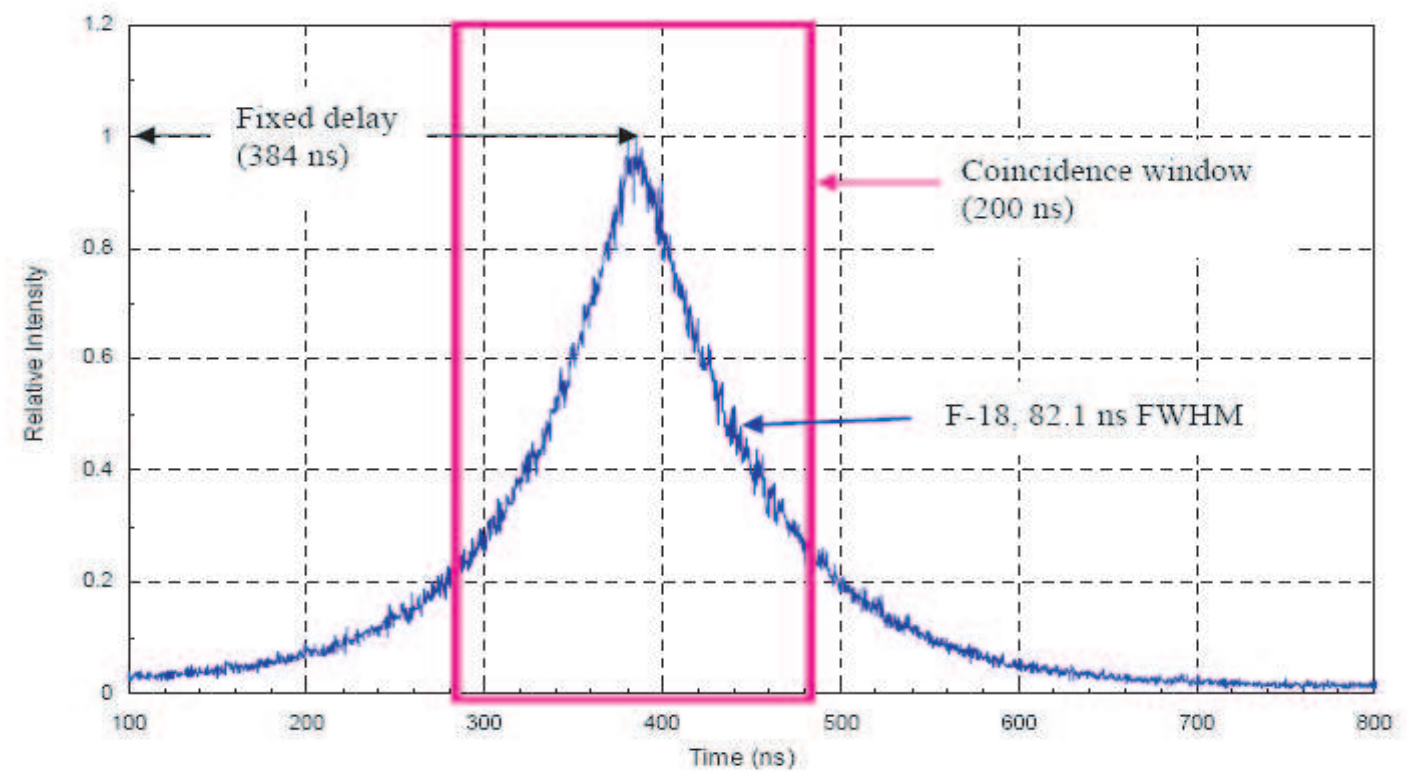

Figure 4.5: Coincidence measurement performed at the University of Michigan using a collimated ${ }^{18} \mathrm{~F}$ line source at the center of the field of view. Note: The $200 \mathrm{~ns}$ coincidence timing window was chosen for the previous small animal PET prototype. A 300 ns timing window was chosen for the setup described in the document. Image taken from [44].

be recorded. Without the delay, pulses from OSU2 can enter the TDC first and the event would not be recorded, yielding only half of the timing curve. The output of the TDC was fed to a multi-channel analyzer (MCA) that digitized the TDC analog output signal.

A timing uncertainty of 82.1 ns FWHM was measured using a low activity ${ }^{18} \mathrm{~F}$ collimated line source in the center of the FOV [7]. The poor timing resolution is due to a combination of variations in charge collection times due to depth of interaction effects in the silicon, a constant threshold voltage in the VATAGP3 trigger logic, and 
the variation in pulse rise times due to the wide energy range of the Compton recoil electron $(30-340 \mathrm{keV})$ for $511 \mathrm{keV}$ gamma rays [8].

For this study a 300 ns coincidence timing window was chosen in order to decrease data collection times. A large timing window will not degrade the final image resolutions in this study because low activity point sources, on the order of $50 \mu \mathrm{Ci}$, are used and are positioned entirely within the detection plane. Point sources, unlike line sources, do not need to be collimated because there is no need to remove background triggers caused by annihilation events occurring outside of the detection plane which can saturate the system with events that can never have a corresponding coincidence trigger due to the geometry of the setup. The expected number of random coincidences can be found by the equation

$$
r=2 \tau s_{1} s_{2}
$$

where $\mathrm{r}$ is the number of expected random events, $\tau$ is the coincidence timing window, $s_{1}$ is the singles rate from detector 1 , and $s_{2}$ is the singles rate from detector 2 . From equation 4.1, the calculated random coincidence rate for our setup is $0.014 \mathrm{~Hz}$, using coincidence timing window $\tau=300 \mathrm{~ns}$ and singles rates $s_{1}=s_{2}=150 \mathrm{~Hz}$ for a $50 \mu \mathrm{Ci}$ point source located at the center of the FOV. It takes approximately 90 minutes to collect a complete data run of 36,000 coincidence events for ${ }^{68} \mathrm{Ga}$. For this data we expect to record 8 random events. Thus, the number of recorded random events is negligible.

\subsubsection{Coincidence Timing Set-up}

The purpose of this section is to describe the coincidence timing electronics used to determine if either an event has occurred in both silicon detectors within the 


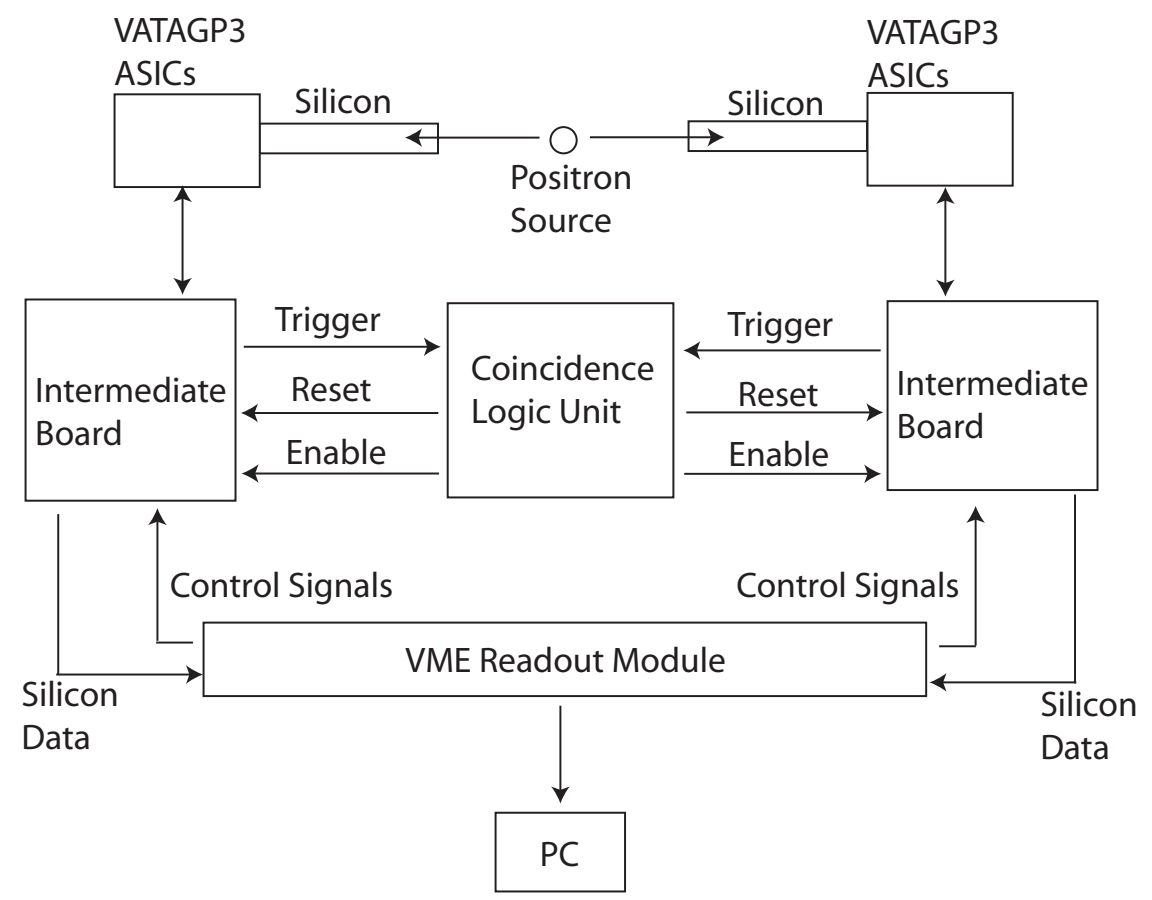

Figure 4.6: Block diagram of the small animal PET prototype readout electronics.

coincidence timing window or if only one detector registered an event and needs to be reset. For the first part of this discussion, the coincidence timing unit is treated as a black box; however, a more detailed explanation of the timing electronics is given after the more general explanation of the timing readout structure is explained.

The VATAGP3 chips, discussed in section 3.4.1 interface with an intermediate electronics board that sends and receives NIM level logic signals with a coincidence timing unit and communicates with the VME readout module. When a VATAGP3 chip detects an event above the threshold voltage, the intermediate board generates a standard NIM logic level trigger signal. This trigger signal is sent to the coincidence timing logic in order to determine if an event occurred in coincidence with the second silicon detector. A $300 \mathrm{~ns}$ coincidence timing window was chosen due to the poor 
timing resolution of the VATAGP3 chips as discussed in section 4.3. In the event that a trigger signal from the second silicon detector does not reach the coincidence module within the $300 \mathrm{~ns}$ timing window, then the detector that triggered is sent a reset signal, returning the system to its initial state. If the two trigger signals arrive within the coincidence timing window, readout signals are sent out to the two intermediate boards initiating the readout cycle.

The energy from each of the 512 pads of the two silicon detectors is readout via the intermediate board/VME interface and stored on a local PC for post processing analysis. The role of the intermediate boards is to the supply the threshold voltage, power, and the current and voltage biases to the VATAGP3 chips. The intermediate board also interfaces with the coincidence logic unit as previously discusses.

At the start of a run, the VME board loads the masks (so that no triggers from broken channels are accepted), thresholds, and initializes the VATAGP3 chips for data collection by communicating through the intermediate board. During the readout cycle the VME board receives the trigger signal and sends out the sample and hold flag that waits for information from the coincidence logic unit for readout instructions. The readout timing is controlled by the VME board (1.2 $\mathrm{MHz}$ clock cycle). The analog to digital converters on the VME board digitize the signals prepared by the intermediate board. Once the event has been digitized it is stored in a file on a local $\mathrm{PC}$ for later analysis.

The VME board and the PC communicate through the VMEDAQ software program that is used to set the masked channels, thresholds, and data readout mode [47]. The VMEDAQ software allows data to be collected in serial mode (all channel energies recorded per event), sparse mode (only the energy of the channels above 


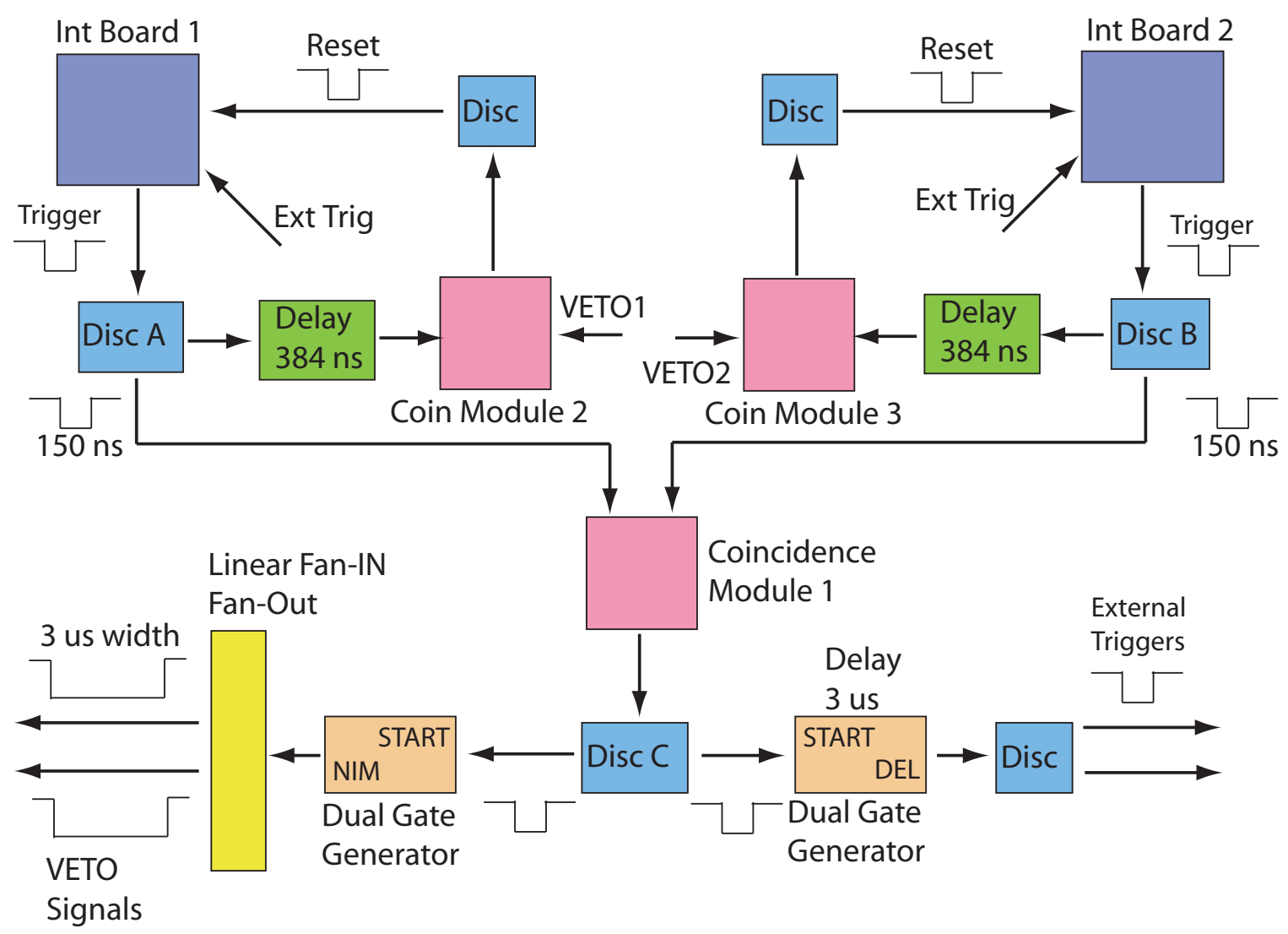

Figure 4.7: Small animal PET coincidence timing set-up

threshold are readout), or sparse plus adjacent (the energy of the channels above threshold and their nearest neighbors are readout). In this analysis only the serial data readout mode is used.

\subsection{Coincidence Timing Module Details}

The coincidence timing unit that was represented as a black box in the previous section is actually not a single module at all, but is composed of many NIM (Nuclear Instrumentation Methods) modules, each of which performs a small function. A detailed schematic of the coincidence timing unit is shown in Fig. 4.7. 
The coincidence timing unit interacts exclusively with the two intermediate boards and responds differently corresponding to the type of event evaluated. The two type of events that the coincidence timing unit must process are coincidence events and events where only one detector has triggered. For coincidence events, the coincidence timing unit must output external triggers to send back to the intermediate board to tell the system to record the data. For events where only one detector triggered, the coincidence logic unit must send a reset signal back to the detector that triggered. The following paragraph outlines the response of the coincidence timing unit to these two situations.

The coincidence timing cycle begins when either of the two intermediate boards emit a trigger signal. This trigger signal is split by a discriminator into two identical NIM pulses each 150 ns wide. The overlap of these two 150 ns pulses defines the 300 ns coincidence window. If a coincidence event has occurred then the two outputs from discriminators $\mathrm{A}$ and $\mathrm{B}$ will overlap and coincidence module 1 will send out a pulse corresponding to the overlap of the two input pulses. This pulse is split via discriminator $\mathrm{C}$ into two 100 ns wide pulses. One of these pulses is used to generate two external triggers delayed by $3 \mu$ s (the shaping time of the slow shaper) that are fed back into the intermediate boards to tell the system that a coincidence event has occurred and that the data should be readout. The second pulse from discriminator $\mathrm{C}$ is used to generate an analog VETO signal to send to coincidence modules 2 and 3 , thus stopping the detectors from being reset. However, if a coincidence event has not occurred the detector that sent out the trigger signal needs to be reset. The initial trigger signal from the intermediate board split by either discriminator A or B is sent to either coincidence module 2 or 3 , depending on which detector triggered. This 
signal is delayed by $384 \mathrm{~ns}$ such that there is time to check to see if a coincidence event has occurred. If the VETO signal is not present (i.e. a coincidence event was not detected) a signal is sent back to the intermediate board to reset the detector that triggered, leaving the system open for the next event.

\subsection{Turntable Tilt}

A possible source of resolution blurring can arise if the turntable is tilted with respect to the plane of the detector. Ideally, the turntable and the image plane would be perfectly parallel; however, in practice the planes are likely to be slightly skewed with respect to each other. A tilted turntable plane can translate into a source of resolution blurring. There are two possible tilted turntable geometries, as shown in Figs. 4.8 and 4.9 , and both will yield radically different results to the image resolution.

The first geometry is for a turntable that has a normal vector that always points in the same direction (Fig. 4.8). In this case, both resolution blurring and incorrect sampling of the positron range distribution can occur. As can be seen in the figure, the radial distance of the source in the detection plane will vary as the source is rotated. This is made evident by the different lengths of $R_{1}$ and $R_{2}$ shown in Fig. 4.8. The severity of these effects will depend on the turntable tilt angle $\theta$ measured with respect to the detector plane and the distance of the source from the central rotation axis.

For the second geometry, the normal vector of the turntable plane will precess about the ideal rotation axis (Fig. 4.9). This configuration is preferable to the first geometry because the location of the point source is always at the same radial distance 


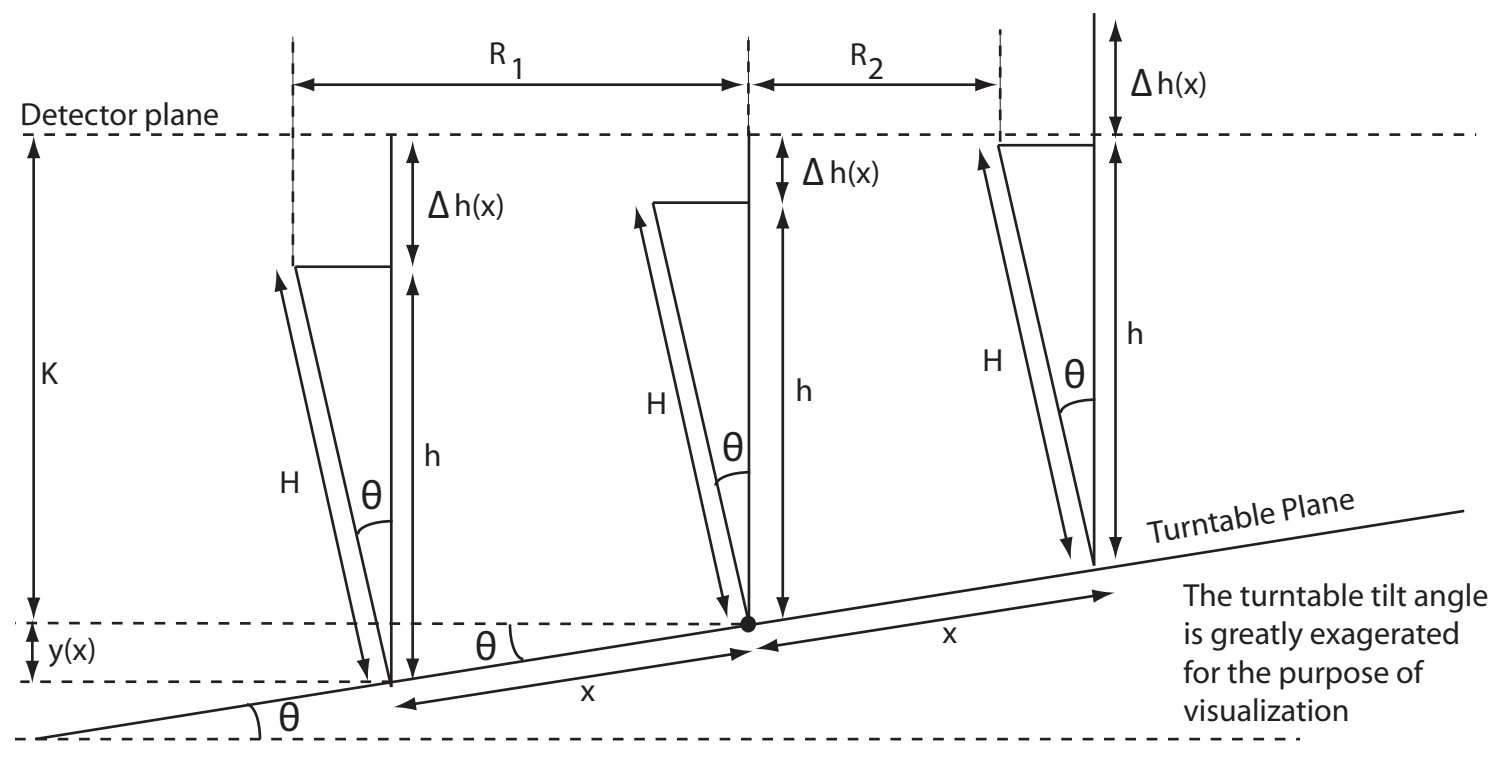

Figure 4.8: Turntable tilt geometry for a tilt where the normal vector to the plane always points in the same direction.

$R$ from the center of the turntable. However, if the detectors are aligned with the source at the origin, then sources located a distance $r$ will be either slightly above or below the detector plane depending on the severity of the turntable tilting angle $\theta$.

The tilt angle of the turntable $\theta$ can be calculated by measuring the deviation of the turntable for each degree of rotation at a known distance from the rotational axis (see Fig. 4.11). This measurement was performed using a dial test indicator device. The dial test indicator probe was position $3.0 \mathrm{~cm}$ away from the rotation axis for both measurements. The results of the measurements are shown in Fig. 4.10. The turntable was put onto a cart and wheeled around the laboratory to see if the turntable deviation was a stable component of the system or if it was susceptible to movement. The agreement between the two measurements show that the changes in the turntable deviation distance is insensitive to device movement. The measured 


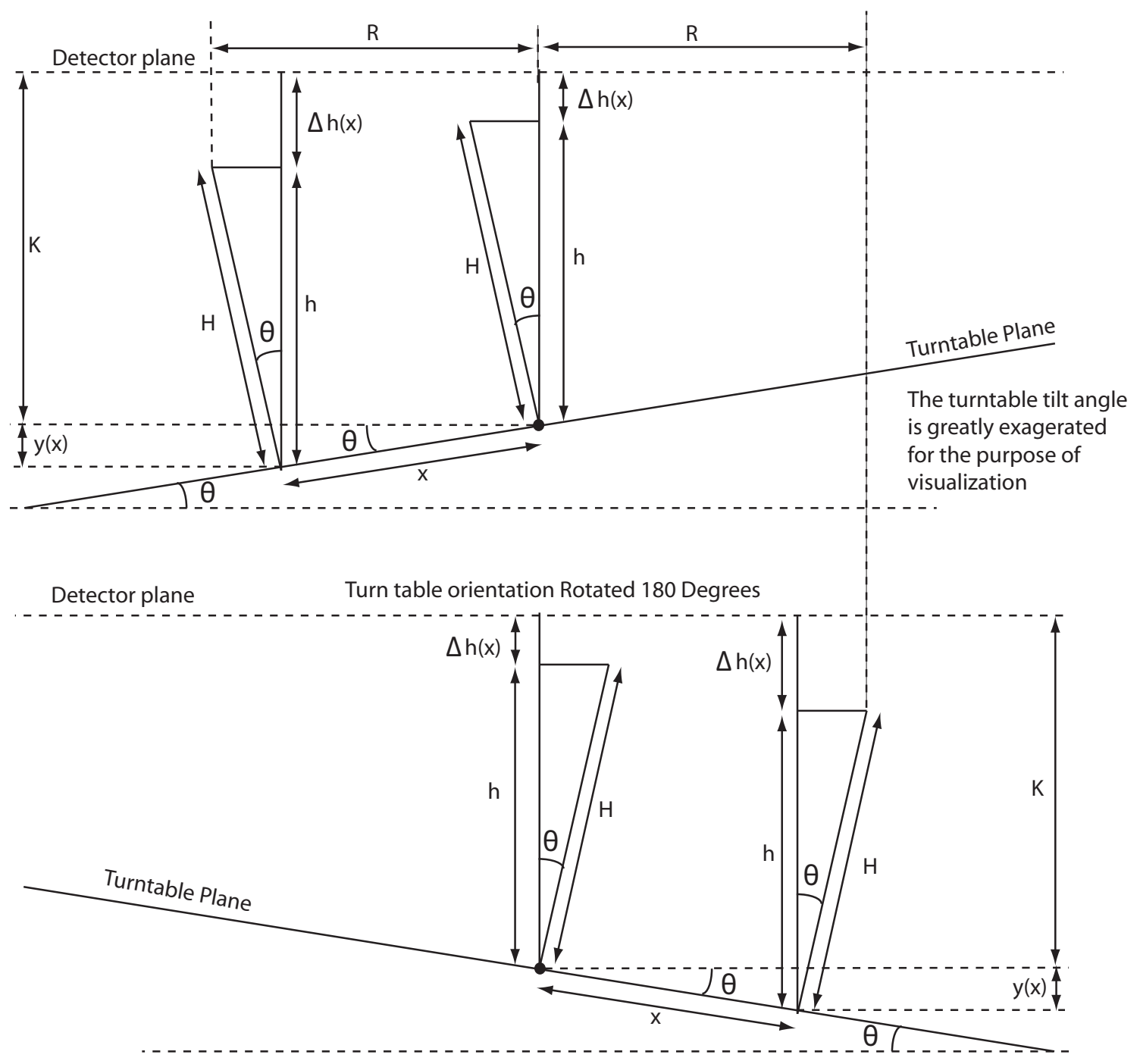

Figure 4.9: Turntable tilt geometry for a tilt where the normal vector to the plane precesses about the ideal rotation axis. This is the turntable geometry of our setup. 


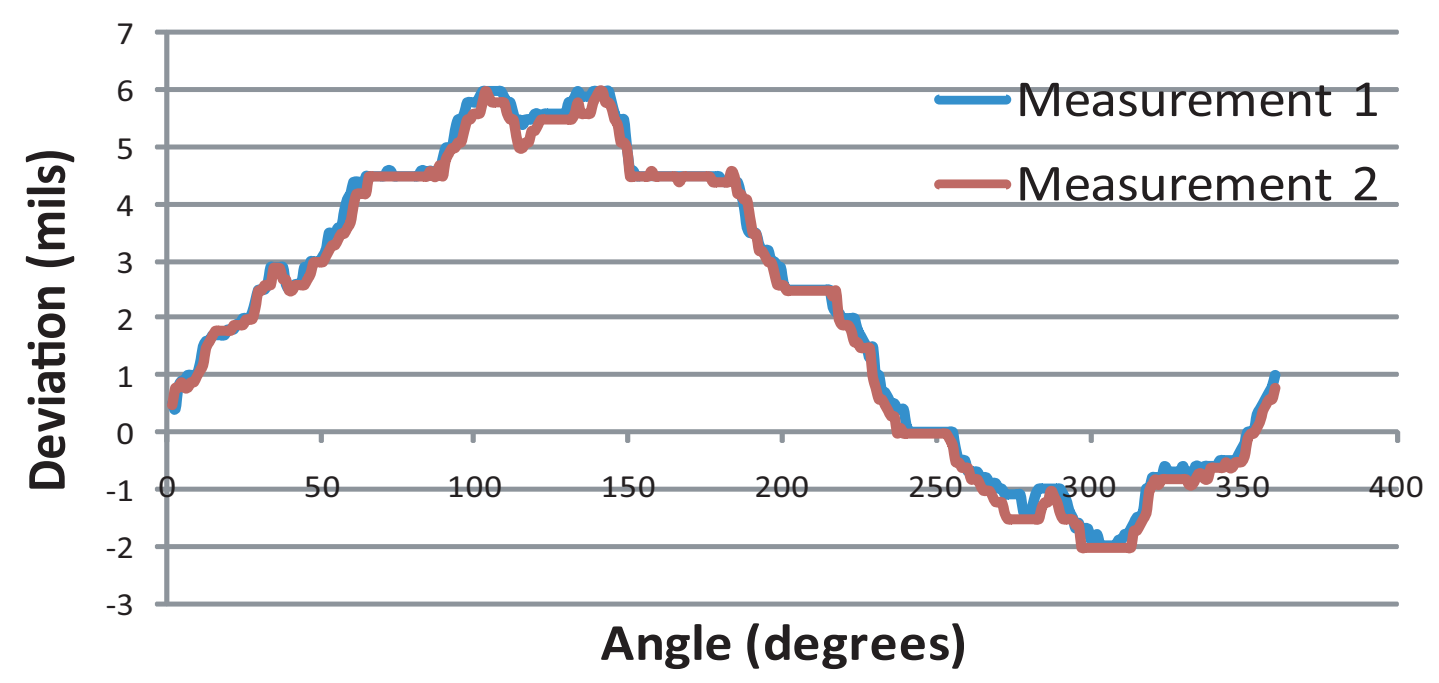

Figure 4.10: Measurement results of the deviation distance $X$ of the turntable.

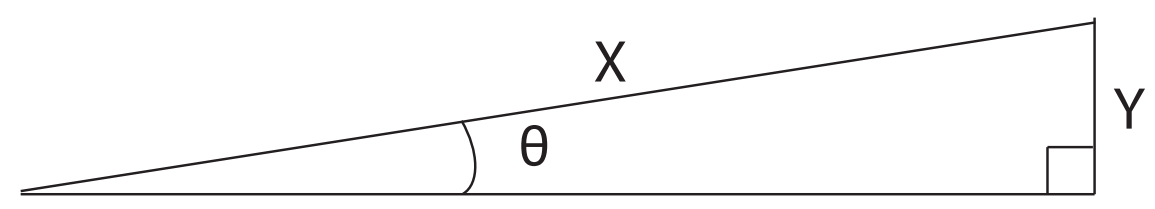

Figure 4.11: Geometry used to calculate the tilt angle.

deviation distance is 8 mils $=0.20 \mathrm{~mm}$. This measurement shows that the turntable is rotating by the second geometry due to the spread in the deviation values. If the turntable was rotated as explained by geometry 1, the deviation for a single point would have been constant.

The tilt angle of the turntable $\theta$ can now be calculated using the turntable deviation distance. The total deviation of the turntable was measured to be $Y=8 \mathrm{mil}=$ $0.2032 \mathrm{~mm}$ measured at $X=30 \mathrm{~mm}$ from the turntable axis. From this measurement 
the angle $\theta$ can be calculated by simply taking the inverse sine of the quotient of the distance from the turntable rotation axis $X$ to the total deviation of the turntable $Y$ (see Fig. 4.11),

$$
\theta=\sin ^{-1}\left(\frac{Y}{X}\right)=0.388 \text { degrees. }
$$

The following geometric analysis is used to determine distance that sources are shifted out of the detector plane as a function of the radial distance $x$ from the center of the turntable. From Fig. 4.9 is can be seen that:

$$
\begin{aligned}
& y(x)+K=\Delta h(x)+h \\
& \Delta h(x)=K+y(x)-h
\end{aligned}
$$

where

$$
h=H \cos \theta
$$

and

$$
y(x)=x \sin \theta
$$

Using equation 4.5 and 4.6 in equation 4.4 and using the approximation that $K \approx H$ for small tilt angles yields

$$
\Delta h(x)=H(1-\cos \theta)+x \sin \theta
$$

Fig. 4.12 is a plot of $\Delta h(x)$. For a source located at $6.0 \mathrm{~mm}$ from the center of the FOV at a height of $675 \mathrm{~mm}$ above the plane of the turntable, $\Delta h(x)=0.06 \mathrm{~mm}$. This is the location for the point source measurements presented in chapter 6 . The sources used in this experiment are $0.25 \mathrm{~mm}$ and $1.0 \mathrm{~mm}$ in height and are aligned within the detector plane positioned along the axis of rotation. Moving the sources off axis to a radial distance of $6.0 \mathrm{~mm}$ results in the $0.25 \mathrm{~mm}$ diameter source still contained 


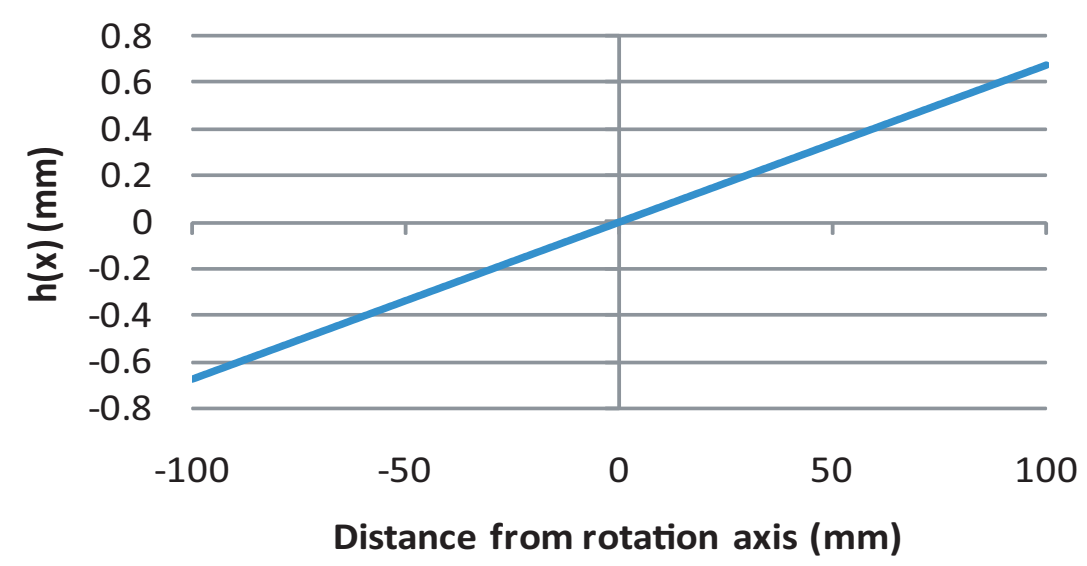

Figure 4.12: Plot of $h(x)$, the offset from the detector plane, for source positions between -100 and $100 \mathrm{~mm}$ radius.

entirely within the detection plane. For the $1.0 \mathrm{~mm}$ source, over 90 percent of the source is still located within the detection plane, and as a result the measurement of the positron range will still be through the center of the distribution. This analysis demonstrates that the measured positron resolution is not greatly affected by the turntable tilt.

\subsection{Aligning the silicon detectors}

The two silicon detectors are each secured to precision level mounts that are used to position the detectors into the same $1.0 \mathrm{~mm}$ thick plane. The height of each silicon detector can be raised and lower with high precision through the use of a screw in the base of the mount. Once the silicon detectors are aligned to the correct height, the pitch of the silicon can be adjusted by dual leveling screws. The two "parallel" plates are bound together by high durability rubber bands and three corners of the 
apparatus rest on two $0.5 \mathrm{~cm}$ diameter and $1.0 \mathrm{~cm}$ diameter marble balls that allow the upper plane to pivot. The upper plane is connected to the box containing the silicon detector. A laser beam is used to confirm that the two silicon detectors are aligned to the same plane and at the same height as the positron source.

In order to confirm that the two silicon detectors are actually in the source plane, coincidence data between the two detectors is taken with the source in various angular positions. The coincidence rate of the source is recorded at each position. If the detectors are truly in the plane of the source the coincidence rate between the two silicon detectors as a function of angular position of the source should trace out a symmetric sine wave. For every data run the coincidence rate was recorded to ensure proper alignment. An example measurement of the frequency versus turntable angle is shown in Fig. 4.13 for a source located at $6 \mathrm{~mm}$ from the center of the FOV. The maxima in the plots are when the source is at its closest approach to either of the silicon detectors. The minima occur when the source is halfway between the detectors.

Figure 4.14 is a plot of the number of hits versus channel number for OSU1 (top) and OSU2 (bottom) for both simulated and measured data. The excellent agreement between simulated and real data is further evidence that the detectors are aligned properly.

After the two silicon detectors have been adjusted to be within the same $1.0 \mathrm{~mm}$ thick source plane, coincidence data is collected in eighteen, 20 degree increments. As discussed in the following chapter, this angular sampling is sufficient for full angular sampling of the data. The data is stored in eighteen compressed data format files, one for each sampling angle. 


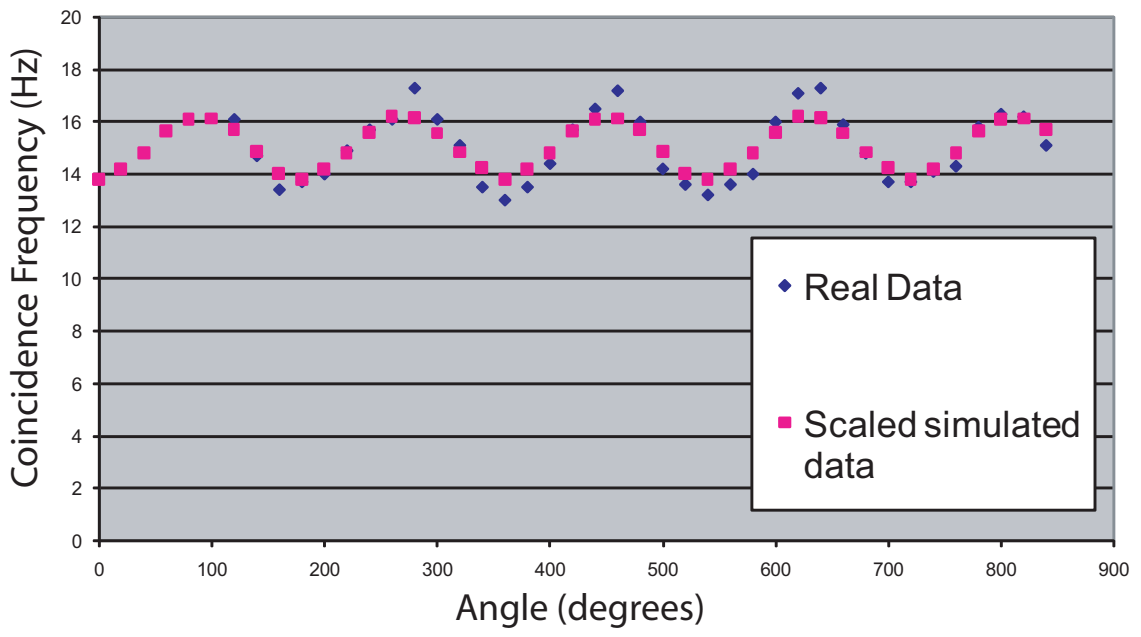

Figure 4.13: Coincidence frequency versus turntable angle used to confirm correct detector alignment.

\subsection{Post processing the data}

The goal of the post processing step is create a list of paired $\mathrm{x}$ and $\mathrm{y}$ positions corresponding to the locations of the annihilation photons in the two silicon detectors taking into account the angular position of the source. This is accomplished by performing a transformation on the data that creates a set of $\mathrm{x}$ and $\mathrm{y}$ positions analogous to data collected from a full detector ring. This transformation is accomplished in two steps. The first step finds the channels that the annihilation photons interacted in for each detector. The second step performs a transformation to rotate the $\mathrm{x}$ and y positions of the pads taking into account the data collection angle $\theta$.

In the first step, a simple channel-by-channel pedestal subtraction is applied to each event. As previously argued, the energy deposited in the silicon detector is only 


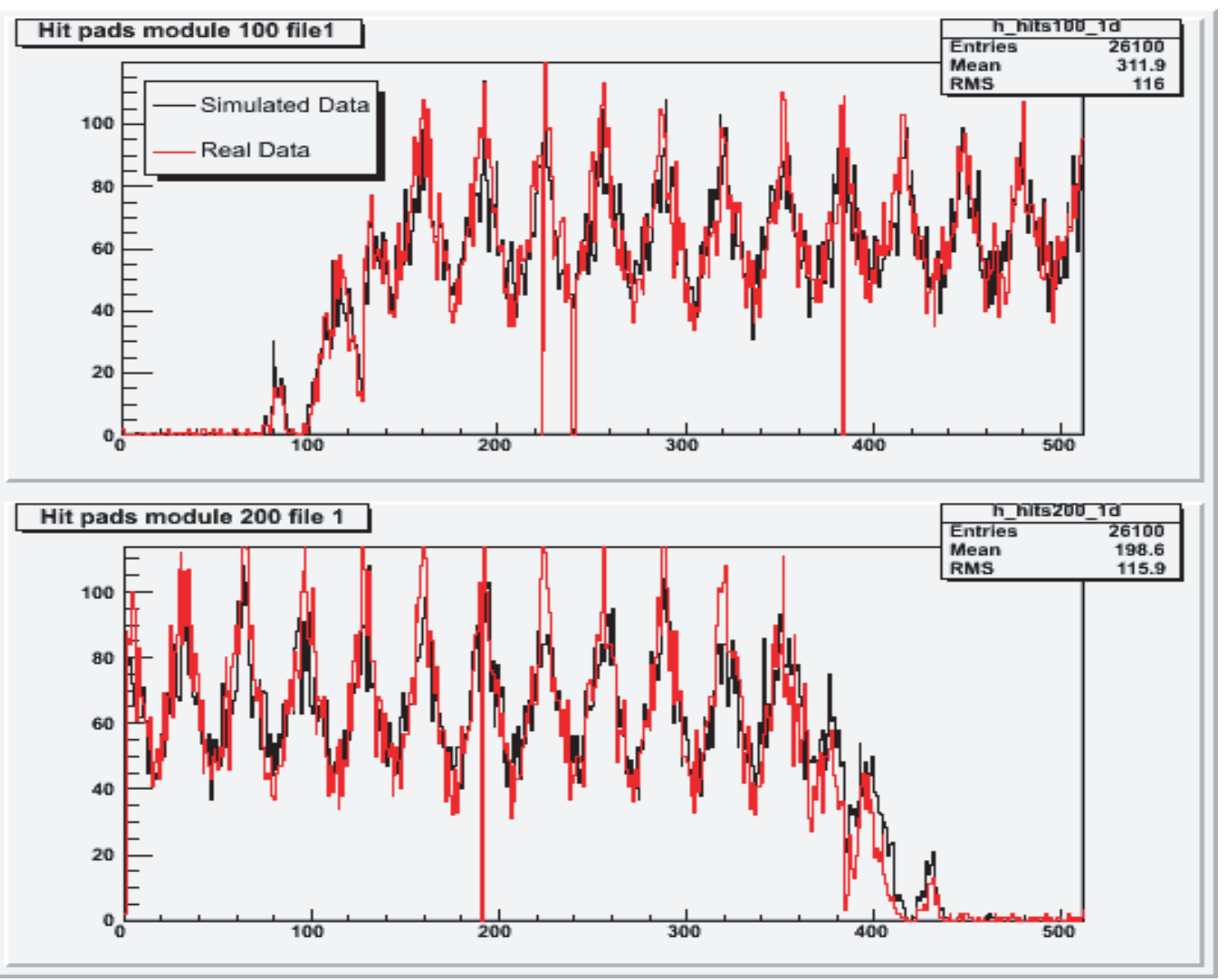

Figure 4.14: Comparison of the hit map between simulated and real data for the source at angle 60 degrees. The excellent agreement between the simulated and real data is further evidence of a properly aligned system. 


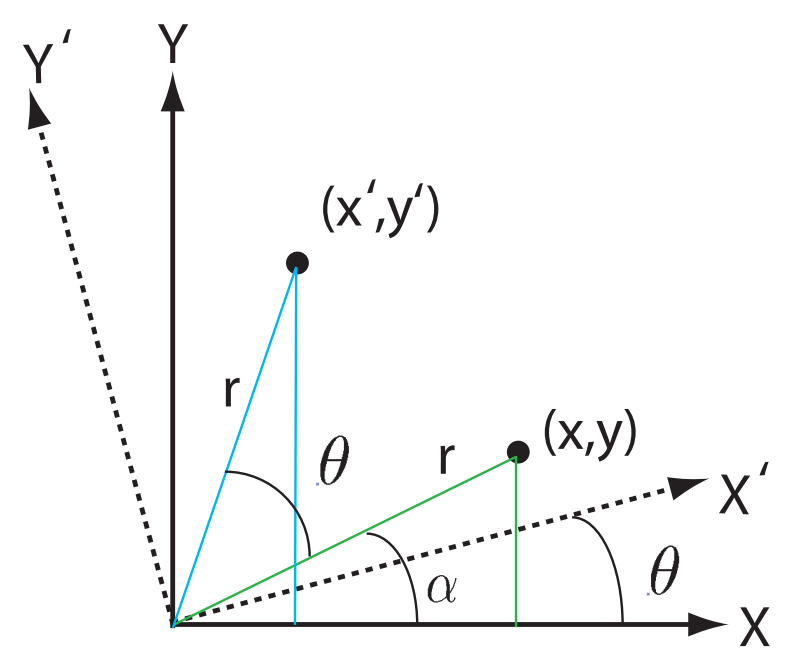

Figure 4.15: Diagram of source rotation transformation. The primed variables have been rotated by the angle $\theta$.

used to determine the channel in which an event occurred. For this reason a commonmode-noise and gain correction is not applied to the data. The pad number is then compared to a look-up table and an $\mathrm{x}$ and $\mathrm{y}$ position is assigned. The exact position within the $1.4 \mathrm{~mm} \times 1.4 \mathrm{~mm}$ area pad where the annihilation event occurred is not known. Instead of simply using the center of the pads for the $\mathrm{x}$ and $\mathrm{y}$ annihilation positions, a random position is picked within the pad using two standard random number generator (one for $\mathrm{x}$, one for $\mathrm{y}$ ). This avoids introducing streaking artifacts into the data.

The second step takes the pair of $\mathrm{x}$ and $\mathrm{y}$ annihilation positions determined in step one and performs an angular transformation to take into account the turntable angle $\theta$. Figure 4.15 is a diagram of the angular transformation process where $r$ is the radial position of the point, $\theta$ is the angular shift, $\alpha$ is the original angular position of the point $(x, y)$ prior to the angular transformation, and $\left(x^{\prime}, y^{\prime}\right)$ is the transformed 
coordinate. The transformed coordinate can be expressed by

$$
\begin{gathered}
x^{\prime}=r \cos (\alpha+\theta) \\
y^{\prime}=r \sin (\alpha+\theta)
\end{gathered}
$$

which can be rewritten as

$$
\begin{gathered}
x^{\prime}=r \cos \alpha \cos \theta-r \sin \alpha \sin \theta \\
y^{\prime}=r \sin \alpha \cos \theta+\cos \alpha \sin \theta .
\end{gathered}
$$

Using the relations

$$
\begin{aligned}
& x=r \cos \alpha \\
& y=r \sin \alpha
\end{aligned}
$$

$x^{\prime}$ and $y^{\prime}$ become

$$
\begin{aligned}
& x^{\prime}=x \cos \theta-y \sin \theta \\
& y^{\prime}=x \sin \theta+y \cos \theta
\end{aligned}
$$

Thus, the rotation transform matrix is given by

$$
\left(\begin{array}{l}
x^{\prime} \\
y^{\prime}
\end{array}\right)=\left(\begin{array}{cc}
\cos \theta & -\sin \theta \\
\sin \theta & \cos \theta
\end{array}\right)\left(\begin{array}{l}
x \\
y
\end{array}\right) .
$$

Fig. 4.16 is a $2 \mathrm{D}$ histogram of the final $x^{\prime}$ and $y^{\prime}$ transformed annihilation positions for a full set of data. Fig. 4.17 is a histogram of the penetration depth of each photon interaction.

Another simulation was performed that extended the silicon such that the exponetial fall off of the number of interactions in the silicon could be seen. This distribution was fit to a Gaussian of the form

$$
I=I_{0} e^{-\mu x} .
$$




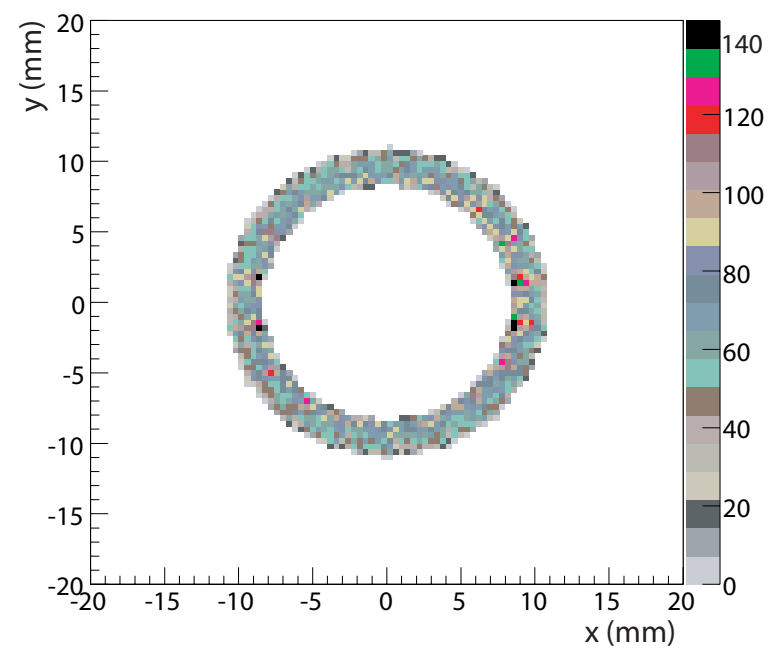

Figure 4.16: 2D density plot of the transformed $x^{\prime}$ and $y^{\prime}$ photon annihilation positions.

where $\mathrm{I}$ is the intensity of the number of events interacting at depth $\mathrm{x}, I_{0}$ is the maximum intensity, and $\mu$ is the silicon attenuation coefficient. The extracted fit value for the attenuation coefficient $\mu$ is $0.202 \pm 0.003 \mathrm{~cm}^{-1}$ which is in excellent agreement with the accepted value of $0.20 \mathrm{~cm}^{-1}$.

The full list of angular transformed $\mathrm{x}$ and $\mathrm{y}$ positions are used as the input for the generation of a sinogram, which is used as the input for the maximum-likelihood estimation-maximization (ML-EM) reconstruction algorithm discussed in chapter 5 . 


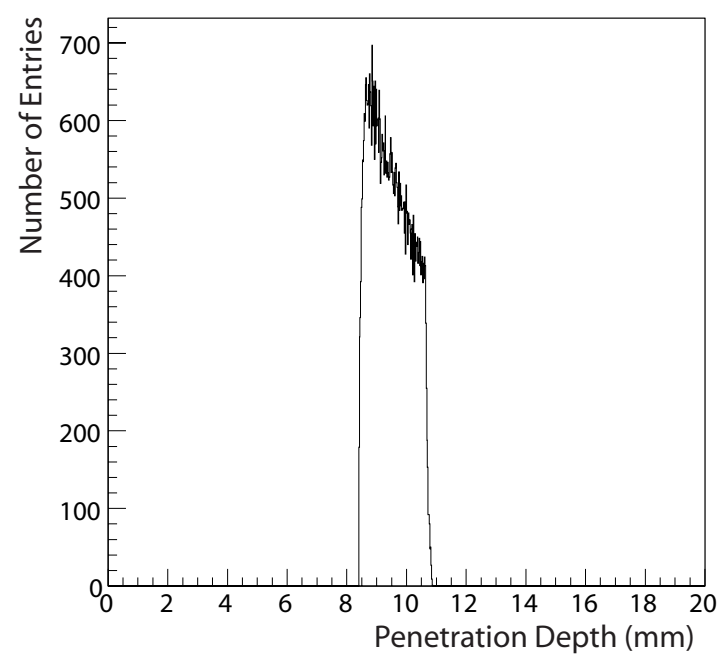

Figure 4.17: Histogram of the penetration depth of each photon interaction calculated using $\sqrt{x^{2}+y^{2}}$.

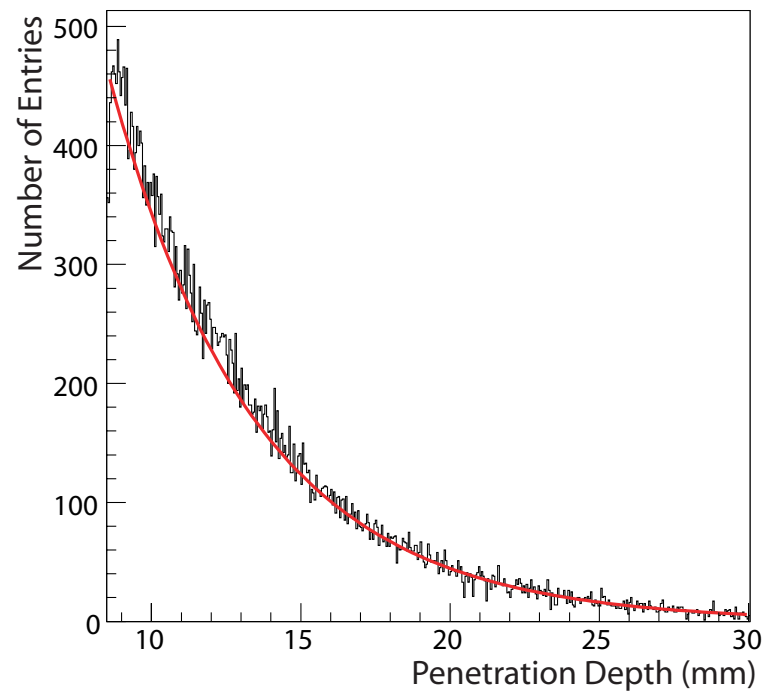

Figure 4.18: EGS4 simulated silicon penetration depth fit to an exponential. The decay parameter of the exponential is the attenuation coefficient which is $0.202 \mathrm{~cm}^{-1}$ extracted from the fit which is in excellent agreement with the the accepted value of $0.20 \mathrm{~cm}^{-1}$. 


\section{CHAPTER 5}

\section{Image Reconstruction}

The purpose of any image reconstruction algorithm is to determine an estimate of the true source distribution $f(x, y)$ from measured data. There are two main types of images reconstruction techniques: analytical and iterative. Analytical reconstruction techniques have the advantage of short processing time to reconstruct the images; however, these images tend to have significant blurring that makes it difficult to distinguish fine detail because the mathematics assume noise free data [48]. Iterative reconstruction methods have the advantage that the geometry, the physics of the detection system, and both random and statistical noise can be modeled and potentially be corrected for. Iterative reconstruction algorithms, equipped with a known model of the imaging system, find the most likely image that matches the collected data. Drawbacks for iterative reconstruction techniques include longer processing times and statistical noise introduced by the reconstruction itself. However, iterative reconstruction techniques have gained popularity in the past few decades due to advances is processing power. In this study, the maximum likelihood expectation maximization algorithm (ML-EM) is used. The ML algorithm [49], originally developed for statistical mathematics, was introduced by Shepp and Vardi for emission tomography in their 1982 paper [50]. 
A comparison between the image quality of a filtered back projection image and an ML-EM image after twenty iterations is shown in Fig. 5.1. Both images are reconstructions of three simulated $0.25 \mathrm{~mm}$ diameter ${ }^{22} \mathrm{Na}$ point sources in water at $0 \mathrm{~T}$. The image on the left is reconstructed using the analytic filtered back projection method [48]. The image on the right is of the same source distribution but is reconstructed with the ML-EM algorithm terminated after twenty iterations. Note that the filtered back projection image possess worse image resolution and streaking artifacts that are not present in the ML-EM image. The ${ }^{22} \mathrm{Na}$ point source are more easily distinguished for the ML-EM image.
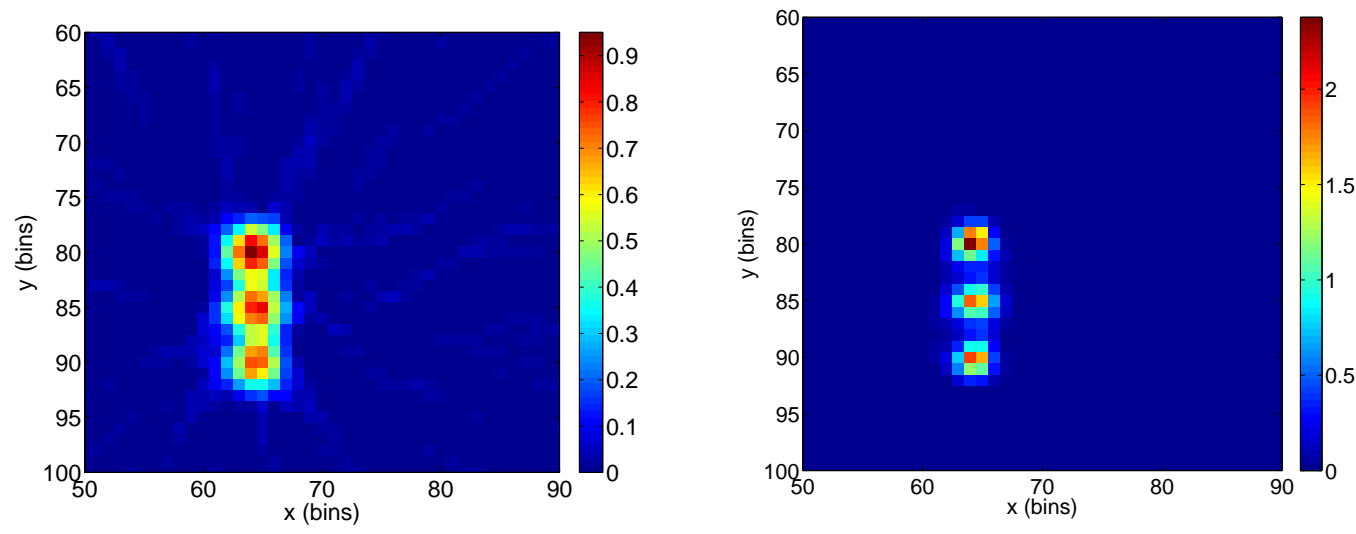

Figure 5.1: Example of EGS4 simulated data of three ${ }^{22} \mathrm{Na}$ point sources at $0 \mathrm{~T}$ in water reconstructed with the analytical filtered back projection method (left) [48] and 20 iterations of the ML-EM algorithm.

Only iterative reconstruction algorithms are used in this analysis, and thus will be the focus of this chapter. This chapter describes both 2D planar maximum likelihood expectation maximization algorithm (ML-EM) and 3D ML-EM algorithms that statistically correct for the range of the positron. 


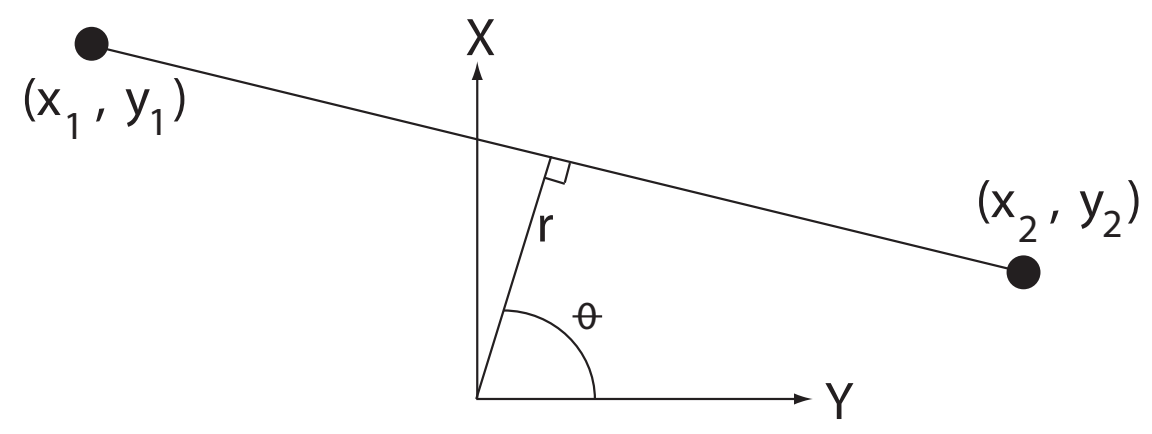

Figure 5.2: Figure illustrating the variables of a sinogram. The distance $r$ is the shortest distance from the origin to the LOR, and $\theta$ is the angle with respect to a user defined axis.

\subsection{Sinograms (Radon Transformation)}

The sinogram is the standard format for organizing PET data that represents the coincidence lines of response (LORs) with the variables $r$ and $\theta$, where $r$ is the shortest distance from the center of the field of view (FOV) to the coincidence LOR and $\theta$ is the angle of $r$ relative to a user defined reference angle (see Fig. 5.2) instead of the two $\mathrm{x}$ and $\mathrm{y}$ photon interaction positions defining the end points of each LOR.

The next series of plots are meant as an illustration of the properties of sinograms. Fig. 5.3 contains sinograms of a ${ }^{22} \mathrm{Na}$ (left) and a ${ }^{68} \mathrm{Ga}$ (right) point sources embedded in water equivalent plastic at $0 \mathrm{~T}$. As can be seen from the figure, a point source traces out half a period of a sine wave. The two point sources are located at the same radius from the center of the FOV as indicated by the amplitude of the wave. The sources are also separated by 180 degrees as can be observed by the 180 degree phase shift between the two sinograms. The width of the reconstructed point can also be estimated by examining the width of the sinogram along the radial axis. As expected, the lower 

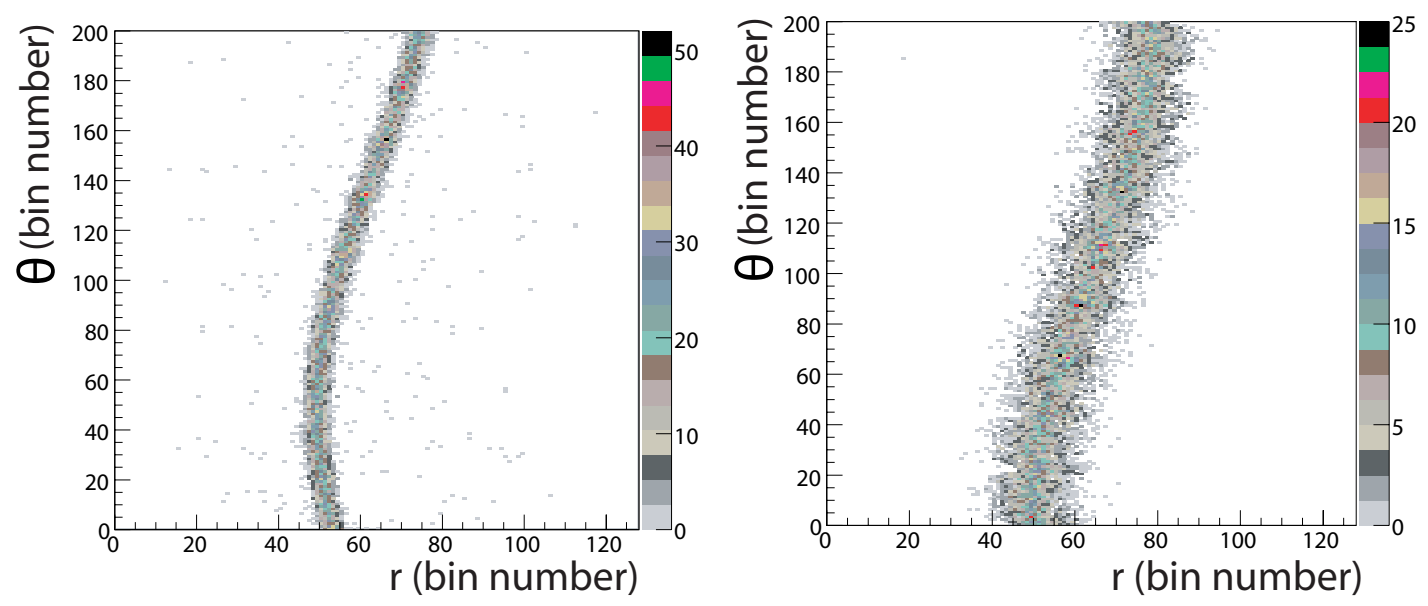

Figure 5.3: Example sinograms of a 0.25 diameter ${ }^{22} \mathrm{Na}$ (left) and 1.0 diameter ${ }^{68} \mathrm{Ga}$ point source point source located $6 \mathrm{~mm}$ off the center of the FOV embedded in water at $0 \mathrm{~T}$. One can note that the sources will be reconstructed by 180 degrees apart due to the difference in phase of the sinogram shapes.

energy positron emitter ${ }^{22} \mathrm{Na}$ yields a tighter sinogram curve than the higher energy positron emitter ${ }^{68} \mathrm{Ga}$. The isolated events located off the main curve are random or scattered coincidence events. Figure 5.4 is an example of two ${ }^{22} \mathrm{Na}$ point sources both located at a radial distance of $6 \mathrm{~mm}$, but on opposite sides of the center of the field of view.

\subsection{Iterative Image Reconstruction: The ML-EM Algorithm}

The purpose of any image reconstruction algorithm is to determine an estimate of the true source distribution $f(x, y)$ from the measured data. The data in PET systems is stochastic in nature due to the independent random emission of the positrons from 


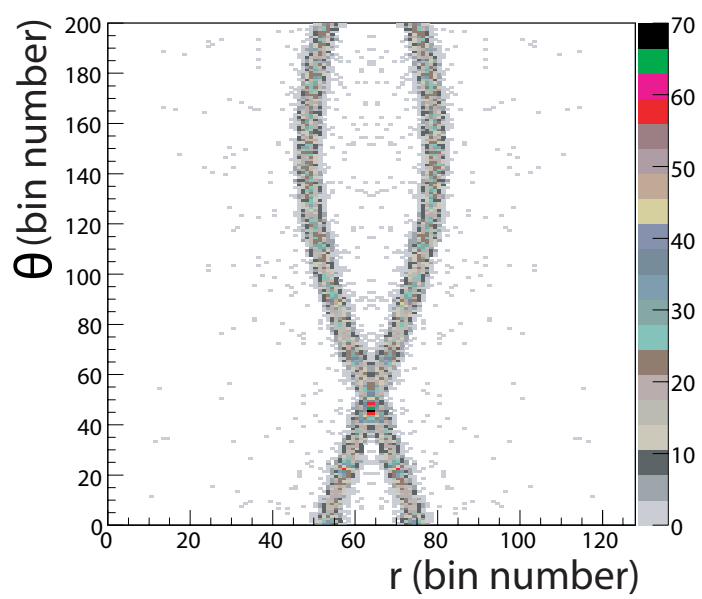

Figure 5.4: Example sinograms of two 0.25 diameter ${ }^{22} \mathrm{Na}$ point source point source located $6 \mathrm{~mm}$ off the center of the FOV embedded in water at $0 \mathrm{~T}$ on opposite sides of the center of the field of view.

the source, the random path of the positron through tissue, and the random photon emission and acollinearity angle. Each detector pair observes only a fraction of the total rate of the Poisson distributed events along the line joining the two detector elements. The mean number of Poisson distributed events $\lambda_{i}$ detected over a given period of time along a line joining detector pair $j$ is given by

$$
\lambda_{j}=\int_{\text {Line }} \int f(x, y) d x d y .
$$

where the integral is over the line joining the detector pair.

The Cartesian $x, y$ coordinates of the detector geometry defining the LORs are an awkward coordinate system for determining the line integral densities for each detector pair. For this reason, a Radon transformation is performed on the data creating a 2D histogram that describes each LOR by the variables $r$ and $\theta$ as described in section 5.1. Transforming the $x, y$ coordinate pairs with a Radon transformation 
yields a sinogram $\mathbf{y}$ containing all of the information of the measured data, where $\mathbf{y}=$ $y(1), y(2), \ldots, y(j), \ldots, y(J)$ correspond to the number of emission lines (projections) measured for each $r_{j}$ and $\theta_{j}$, and $J$ is the total number of bins in the sinogram. Each bin corresponds to the density of a particular LOR. The measured data $\mathbf{y}$ is used in the ML-EM algorithm to estimated the source density $\boldsymbol{\lambda}=\lambda(1), \lambda(2), \cdots, \lambda(I)$ $(I=$ total number of bins in the image space). The discrete estimate of the image distribution $\boldsymbol{\lambda}$ will approach the actual continuous source density $f(x, y)$ by increasing the number of bins in the image space; however, this comes at the cost of increased computation time. There is a point were increasing the number of image bins will not improve the image quality. This occurs when the size of the image bins are much smaller than the dimensions of the detector elements.

Equation 5.1 can be rewritten in the discrete form for use in the ML-EM algorithm. Let $a(i, j)$, known as the system matrix, represent the probability that a photon pair emitted from pixel $i$ is recorded by detector pair $j$. Then the average number of events $\tilde{y}(j)$ emitted from pixel $i$ and detected by detector pair $j$ can be expressed as

$$
\tilde{y}(j)=E[y(j)]=\sum_{i=1}^{I} a(i, j) \lambda(i) .
$$

The above operation is known as a forward projection, and $E[y(j)]$ is the expected, mean value of $y(j)$. The image estimate $\lambda(i)$ is projected into the sinogram space by the system matrix $a(i, j)$ which contains information on the geometry of the system, and may also incorporate corrections for the positron range, random and scattered events, photon acollinearity corrections, etc. 
The probability that the number of events $y(j)$ recorded by detector pair $j$ is a Poisson distribution given by

$$
P(y(j))=\frac{e^{-\tilde{y}(j)} \tilde{y}(j)^{y(j)}}{y(j) !}
$$

where $P(y(j))$ is the probability of detecting $y(j)$ events in detector pair $j$. The probability of collecting a particular data set for a full PET system is simply the product of the probability of detecting $y(j)$ events for each channel,

$$
P_{\text {Total }}=P(y(1)) P(y(2)) \ldots P(y(J))
$$

where $J$ is the total number of detector pairs. Put another way, the likelihood function is a measure of the probability that the measured data set $\mathbf{y}$ was produced by the source distribution $\boldsymbol{\lambda}$ :

$$
L(\boldsymbol{\lambda})=P(\mathbf{y} \mid \boldsymbol{\lambda})=\prod_{j=1}^{J} e^{-\tilde{y}(j)} \frac{\tilde{y}(j)^{y(j)}}{y(j) !} .
$$

The goal of the ML-EM algorithm is to maximize the likelihood function given in equation 5.5; however, it is easier to maximize the $\ln (L(\boldsymbol{\lambda}))$, as both the $\ln (L(\boldsymbol{\lambda}))$ and $L(\boldsymbol{\lambda})$ will converge to the same image when maximized [50]. The $\ln (L(\boldsymbol{\lambda}))$ can be written as

$$
l(\boldsymbol{\lambda})=\ln (L(\boldsymbol{\lambda}))=\sum_{j=1}^{J}[-\tilde{y}(j)+y(j) \ln (\tilde{y}(j))-\ln (y(j) !)]
$$

It is shown by Shepp et al. [50] that by taking the second partial derivatives of equation 5.6 with respect to the $\lambda(i) \mathrm{s}$ (contained in $\tilde{\boldsymbol{y}}$, see equation 5.2 ), that the second derivatives are all negative semidefinite and as a result the function is concave. Shepp et al. then argue that sufficient conditions for $\boldsymbol{\lambda}$ to be a maximizer of a concave function is that the Kuhn-Tucker conditions are satisfied. The Kuhn-Tucker 
conditions are given by [51]

$$
\begin{array}{r}
0=\left.\lambda(i) \frac{\partial l(\boldsymbol{\lambda})}{\partial \lambda(i))}\right|_{\boldsymbol{\lambda}} \\
\left.\frac{\partial l(\boldsymbol{\lambda})}{\partial \lambda(i)}\right|_{\boldsymbol{\lambda}} \leq 0, \text { if } \lambda(i)=0
\end{array}
$$

Solving equation 5.7 for $\lambda(i)$ and using the relation

$$
\boldsymbol{\lambda}^{k+1}=E\left[\ln (\boldsymbol{\lambda}) \mid \boldsymbol{\lambda}^{(k)}, \mathbf{y}\right]
$$

which relates the updated image iteration $\lambda^{(k+1)}(i)$ to the maximum of the expectation value of the actual number of events emitted by each pixel given $\boldsymbol{\lambda}$ and $\mathbf{y}$, yields

$$
\lambda^{(k+1)}(i)=\lambda^{(k)}(i) \sum_{j=1}^{J} \frac{y(j) a(i, j)}{\sum_{i^{\prime}=1}^{I} \lambda^{(k)}\left(i^{\prime}\right) a\left(i^{\prime}, j\right)}, i=1,2, \ldots, I .
$$

Equation 5.10 is the iterative routine used by the ML-EM algorithm which can be rewritten to highlight the various stages of the ML-EM algorithm. Given a previous guess of the image density $\lambda^{k}(i)$ the updated guess of the image density $\lambda^{k+1}(i)$ can be determined by

$$
\lambda^{(k+1)}(i)=C^{(k)}(i) \lambda^{(k)}(i)
$$

where $C^{(k)}(i)$ is a correction factor applied to the previous image estimate. The correction factor is given by

$$
C^{(k)}(i)=\sum_{j=1}^{J} a(i, j) \frac{y(j)}{\tilde{y}^{(k)}(j)} .
$$

$C^{(k)}(i)$ checks to see how closely the current guess of the image density is to the actual measured projection data by comparing the ratio of the measured data $y(j)$ to the current estimate $\tilde{y}^{(k)}(j)$. The sum in equation 5.12 back projects the ratio $y(j) / \tilde{y}^{(k)}(j)$ into the image space using the system matrix $a(i, j)$ on a pixel-by-pixel basis. 


\section{ML-EM Step 1 Uniform Initial Guess

$$
\tilde{y}^{(k)}(j)=\sum_{i=1}^{I} \lambda^{(k)}(i) a(i, j)
$$

a)

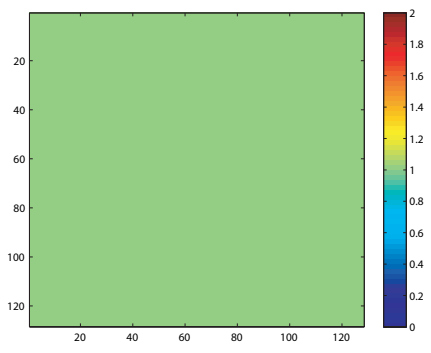

b)

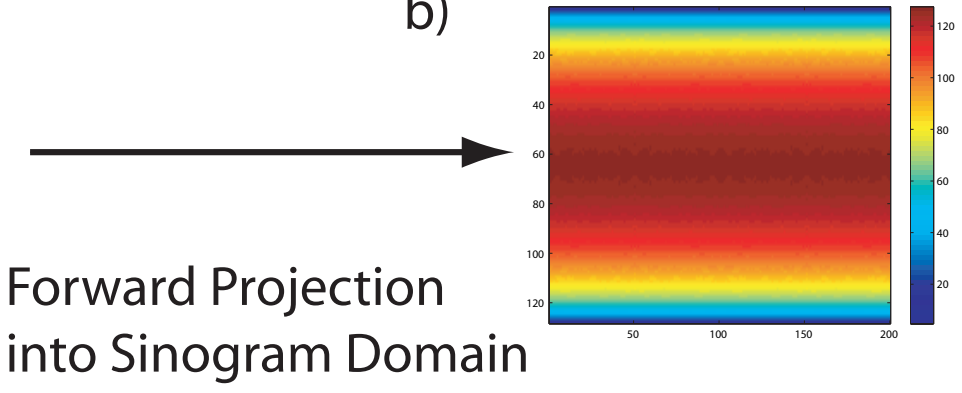

Figure 5.5: The first step of the ML-EM algorithm takes the current image estimate $\lambda^{(k)}$ and forward projects the image into the sinogram space creating $\tilde{y}^{(k)}$.

Figures 5.5 through 5.9 detail the ML-EM algorithm given in equation 5.10 for a single iteration. The first step of the algorithm (Fig. 5.5) takes the current image estimate $\lambda^{(k)}$ and forward projects the image into the sinogram space creating $\tilde{y}^{(k)}$. For this example, the current image estimate is the initial image guess, an unbiased uniform matrix. In step 2 (Fig. 5.6), the measured data $y(j)$ (in sinogram form) is compared to the current estimate of the image sinogram $\tilde{y}^{(k)}$ by a simple, pixelby-pixel division resulting in a estimated sinogram. The sinogram in Fig. 5.6 looks superficially similar to the measured data. Although the shape is the same, the relative intensity of estimated sinogram pixels still differs greatly from the measured data. In step 3 (Fig. 5.7), the estimated sinogram from the previous step is forward projected into the image space by the use of the system matrix $a(i, j)$ to create a temporary image $C^{(k)}$. This image is then used to modify the current estimate $\lambda^{(k)}$ by multiplying each pixel element of the current image estimate $\lambda^{(k)}$ by the temporary 


\section{ML-EM Step 2}

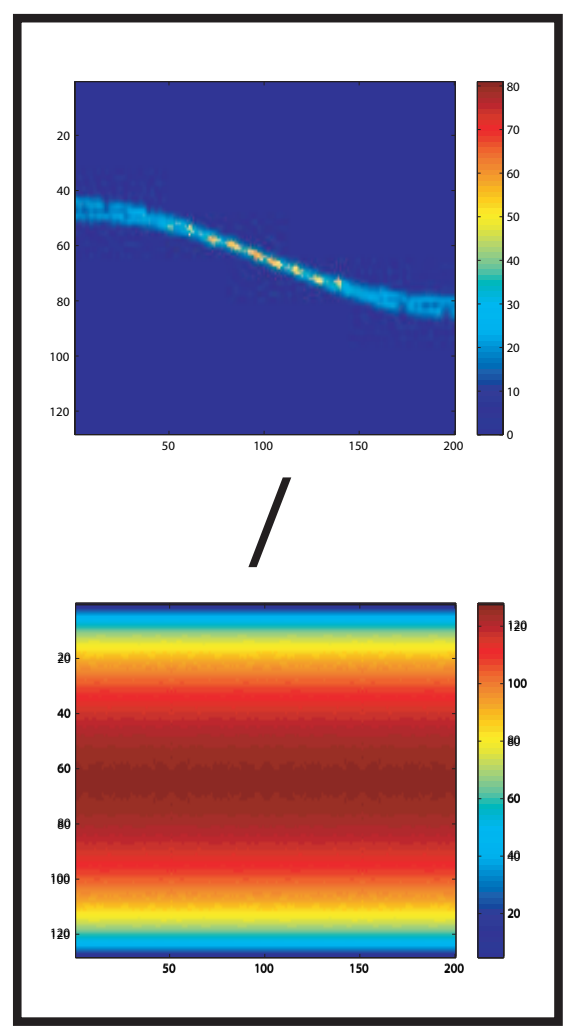

\section{Compare the current guess to the measured data}

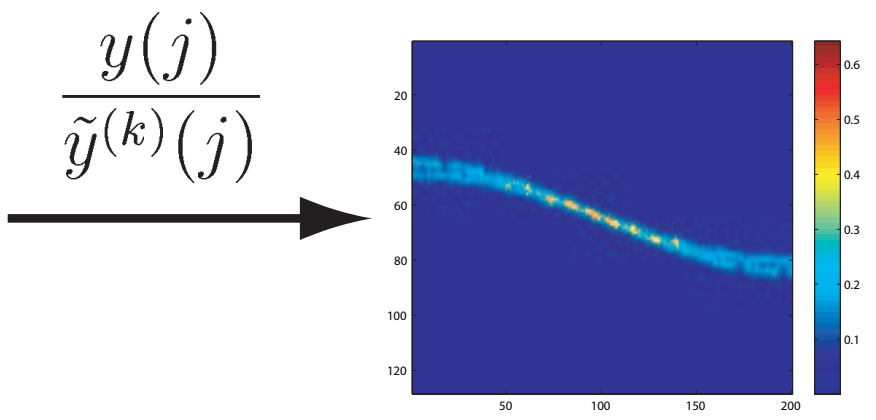

Figure 5.6: In second step of the ML-EM algorithm the measured data $y(j)$ (in sinogram form) is compared to the current estimate of the image sinogram $\tilde{y}^{(k)}$ by a simple, pixel-by-pixel division resulting in a estimated sinogram. 


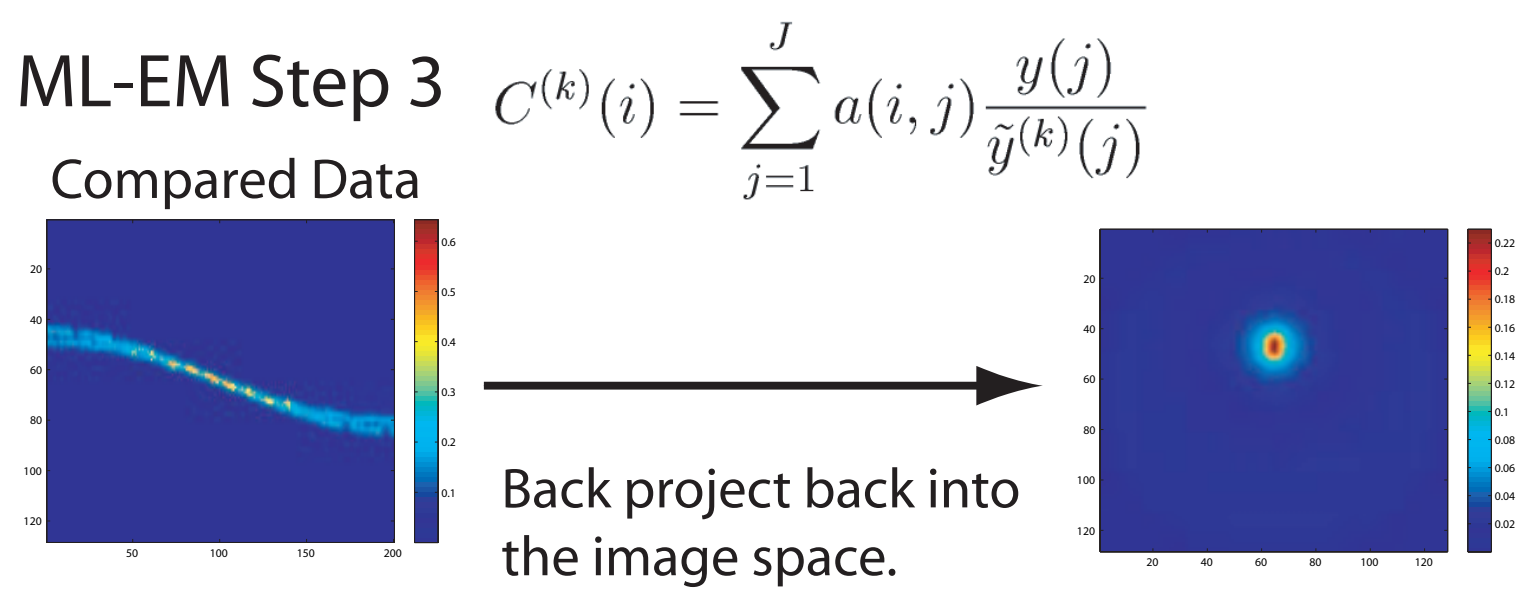

Figure 5.7: In step 3 of the ML-EM algorithm, the estimated sinogram from step 2 is forward projected into the image space by the use of the system matrix $a(i, j)$ to create a temporary image $C^{(k)}$.

\section{ML-EM Step 4}
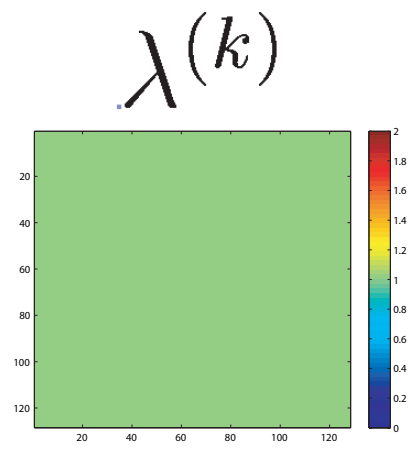
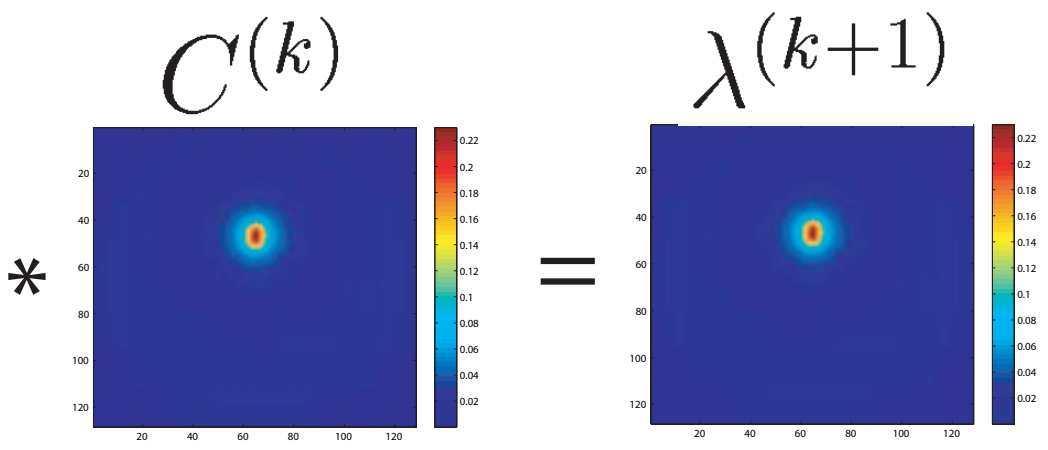

Figure 5.8: The $C^{(k)}$ image is used to modify the current estimate $\lambda^{(k)}$ by multiplying each pixel element of the current image estimate $\lambda^{(k)}$ by the temporary matrix $C^{(k)}$ resulting in the next ML-EM image estimate $\lambda^{(k+1)}$ 


\section{ML-EM Step 1 (again)}

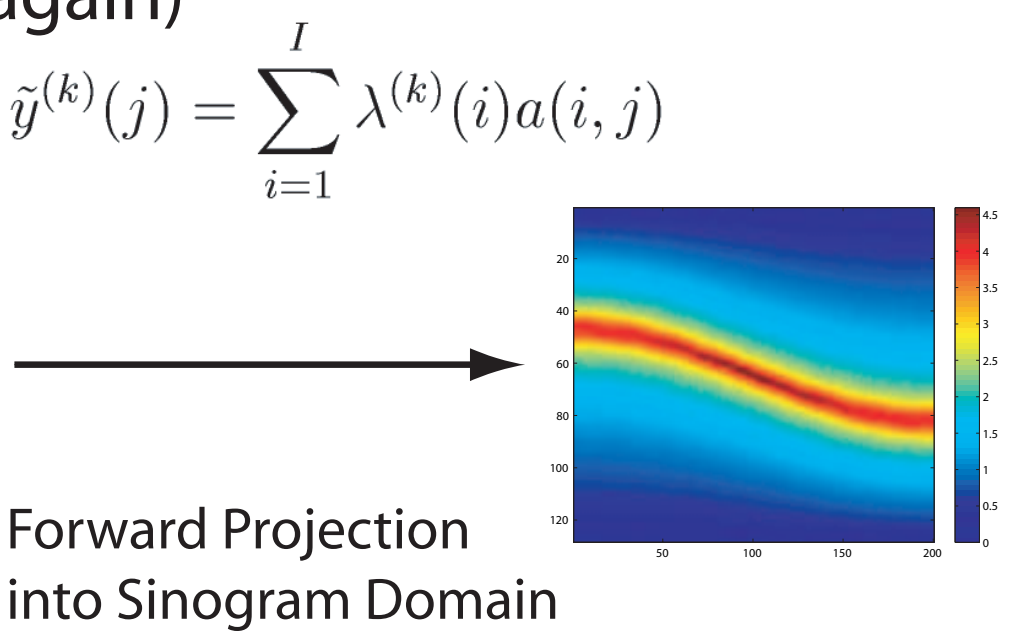

Figure 5.9: The next step of the ML-EM algorithm is the same as step 1, but with a new guess for the image estimate $\lambda^{(k+1)}$. The ML-EM algorithm is repeated until the image has sufficiently converged.

matrix $C^{(k)}$ resulting in the next ML-EM image estimate $\lambda^{(k+1)}$. Fig. 5.9 illustrates the next step of the ML-EM algorithm which is simply to repeat steps one through four, until the image has sufficiently converged (at the discretion of the user).

It is shown in [50] that the likelihood function approaches the maximum likelihood solution with each iteration

$$
L\left(\boldsymbol{\lambda}^{(k+1)}\right) \geq L\left(\boldsymbol{\lambda}^{(k)}\right)
$$

The maximum likelihood solution is reached when $\left|L\left(\boldsymbol{\lambda}^{(k+1)}\right)-L\left(\boldsymbol{\lambda}^{(k)}\right)\right|<\epsilon$ where $\epsilon$ is a small number.

Due to the concavity of $\ln (L(\boldsymbol{\lambda}))$, the maximum of $\boldsymbol{\lambda}$ is a global maximum, and each iteration of the ML-EM algorithm is one step closer to the most likely image $\boldsymbol{\lambda}$ given the measured data $\mathbf{y}$. Figure 5.10 illustrates this point by plotting the FWHM 


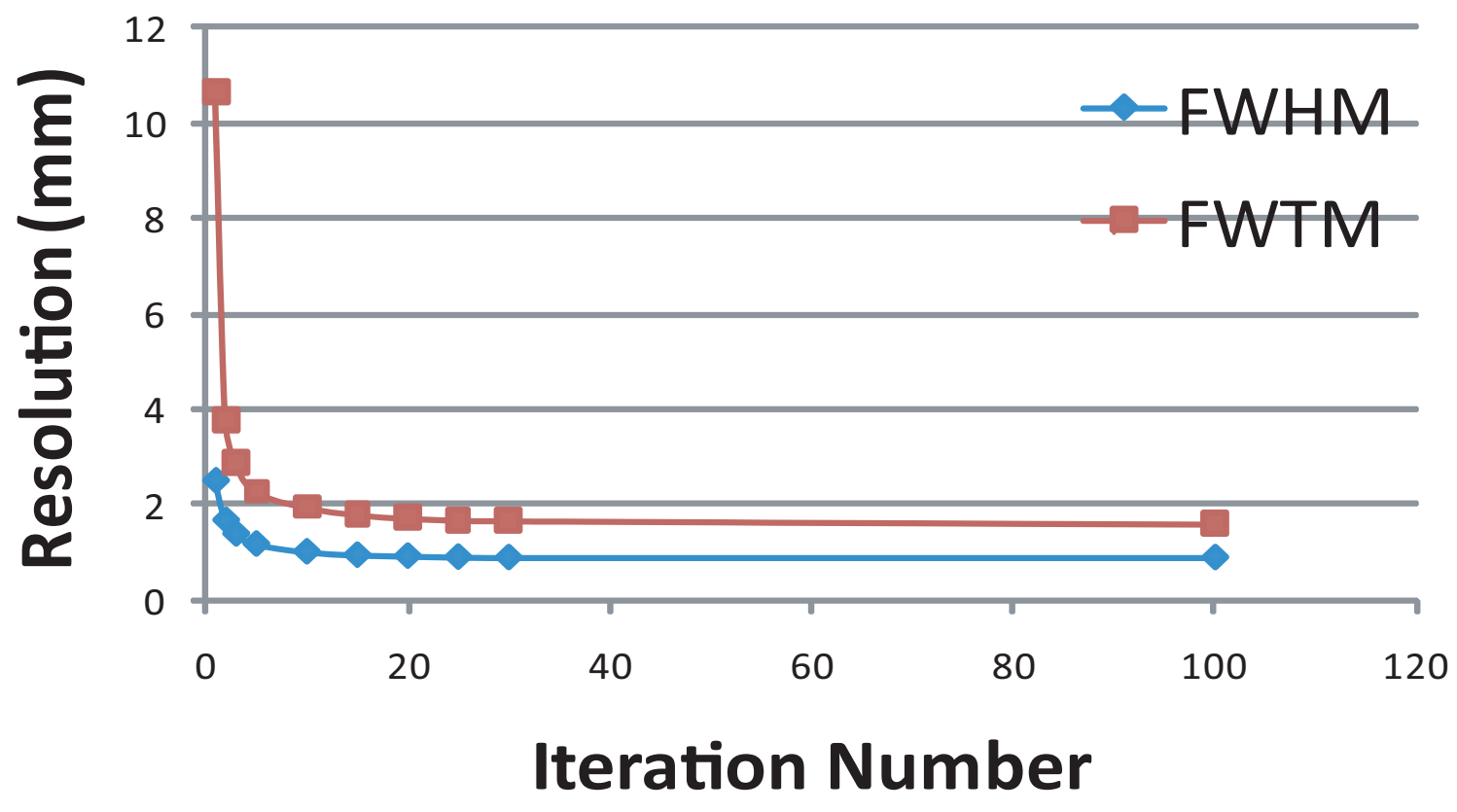

Figure 5.10: FWHM and FWTM resolution verses ML-EM iteration number for a $0.25 \mathrm{~mm}{ }^{22} \mathrm{Na}$ point source embedded in water.

and FWTM resolutions versus iteration number for a $0.25 \mathrm{~mm}$ diameter ${ }^{22} \mathrm{Na}$ source in water. The image is nearly fully converged by the twentieth iteration.

\subsection{D ML-EM Positron Range Correction Algorithm}

This section describes the 3D ML-EM positron range correction algorithm that is used in chapter 7 to investigate the resolution-noise properties of data collected at both $0 \mathrm{~T}$ and $7 \mathrm{~T}$. The $7 \mathrm{~T}$ data is simulated with the magnetic field directed along either the $\mathrm{x}, \mathrm{y}$, or $\mathrm{z}$ axis. The 3D ML-EM algorithm reconstructs the $7 \mathrm{~T}$ data using either one, two, or three orientations of the magnetic field simultaneously. A three dimensional algorithm is needed for this study in order to statistically correct for the 
positron range. The apparatus described in chapter 4 can only collect 2D, planar data. Three dimensional data can be collected by incrementally moving the source out of the detector plane and collecting data at each step; however, this can not be done with high precision. For this reason, simulated data is used for the 3D ML-EM positron range correction algorithm studies.

The 3D ML-EM positron range correction algorithm follows the same general reconstruction steps as the 2D ML-EM algorithm. The differences arise in additional operations that take into account the positron range and the stack of $2 \mathrm{D}$ sinograms that are used to reconstruct a 3D image. Prior to the forward projection step, the current image estimate $\boldsymbol{\lambda}^{(\boldsymbol{k})}$ is Fourier transformed into frequency space. This operation is described by the following equation:

$$
\Lambda^{(k)}(X, Y, Z)=\sum_{l=1}^{N_{x}} \sum_{m=1}^{N_{y}} \sum_{n=1}^{N_{z}} \lambda^{(k)}(x, y, z) w_{N_{x}}^{-(l-1)(X-1)} w_{N_{y}}^{-(m-1)(Y-1)} w_{N_{z}}^{-(n-1)(Z-1)}
$$

where $w_{N}=e^{-2 \pi \imath / N}$ and $N_{x}, N_{y}$, and $N_{z}$ are the number of image space bins in the $\mathrm{x}, \mathrm{y}$, and $\mathrm{z}$ directions, respectively. The Fourier transformation of the image space is multiplied by the frequency equivalent of the positron range function $H_{i}$ containing the positron range distribution for each orientation $i$. The product of the positron range $H_{i}$ and the current image estimate in frequency space $\Lambda^{(k)}$ is then inverse Fourier transformed back into image space using:

$$
\begin{array}{r}
\lambda_{\text {blur }}^{(k)}(x, y, z)=\sum_{i=1}^{N_{\text {or }}} \frac{1}{N_{x} N_{y} N_{z}} \sum_{l=1}^{N_{x}} \sum_{m=1}^{N_{y}} \sum_{n=1}^{N_{z}} \Lambda^{(k)}(X, Y, Z) H_{i}(X, Y, Z) \ldots \\
\ldots w_{N_{x}}^{-(l-1)(x-1)} w_{N_{y}}^{-(m-1)(y-1)} w_{N_{z}}^{-(n-1)(z-1)}
\end{array}
$$

where $N_{\text {or }}$ is the total number of orientations. This series of operations is analogous to performing a three dimensional convolution of the current image estimate with the positron range. The benefit of performing the positron blurring in frequency space is 
that the operation is much faster and thus leads to reduced computational times. The positron range blurred image estimate $\lambda_{b l u r}^{(k)}$ is then forward projected into sinogram space a plane at a time for each orientation $i$ and compared to the simulated sinogram measurements.

As previously stated, simulated data is used for the 3D ML-EM positron range correction algorithm because the current experimental set-up can only accurately collect data in a single plane. This data is created by defining a discrete source distribution in a three dimensional matrix with each voxel of the matrix representing a $0.4 \times 0.4 \times 0.4 \mathrm{~mm}^{3}$ volume. For example, the best estimate of a point source using this geometry is for a single voxel to be given a non-zero value, while the rest of the voxels are set to zero. The simulated sinogram measurements are created by Fourier transforming the "perfect" image into frequency space, blurring the result by the positron range (simulated in EGS4) for each measurement orientation, inverse Fourier transforming the product back into image space, and finally forward projecting the image a plane at a time into the sinogram space for each measurement orientation.

The sinograms that are used to create the images shown in Figs. 5.11 and 5.13 were simulated using the above technique. Figure 5.11 contains two separate images of a ${ }^{68} \mathrm{Ga}$ point source in water at $0 \mathrm{~T}$ positioned in the current plane using the $3 \mathrm{D}$ MLEM positron range correction algorithm (left) and with the positron range correction turned off (right). Analogously, Fig. 5.13 contains the positron range corrected and uncorrected 3D ML-EM images of a ${ }^{68} \mathrm{Ga}$ point source with three orientations of the $7 \mathrm{~T}$ positron range used to reconstruct the image. The range correction can be turned off by using a tight Gaussian distribution for the positron range distribution. In these images, 100,000 Poisson distributed events were used (see section 7.2) and the 

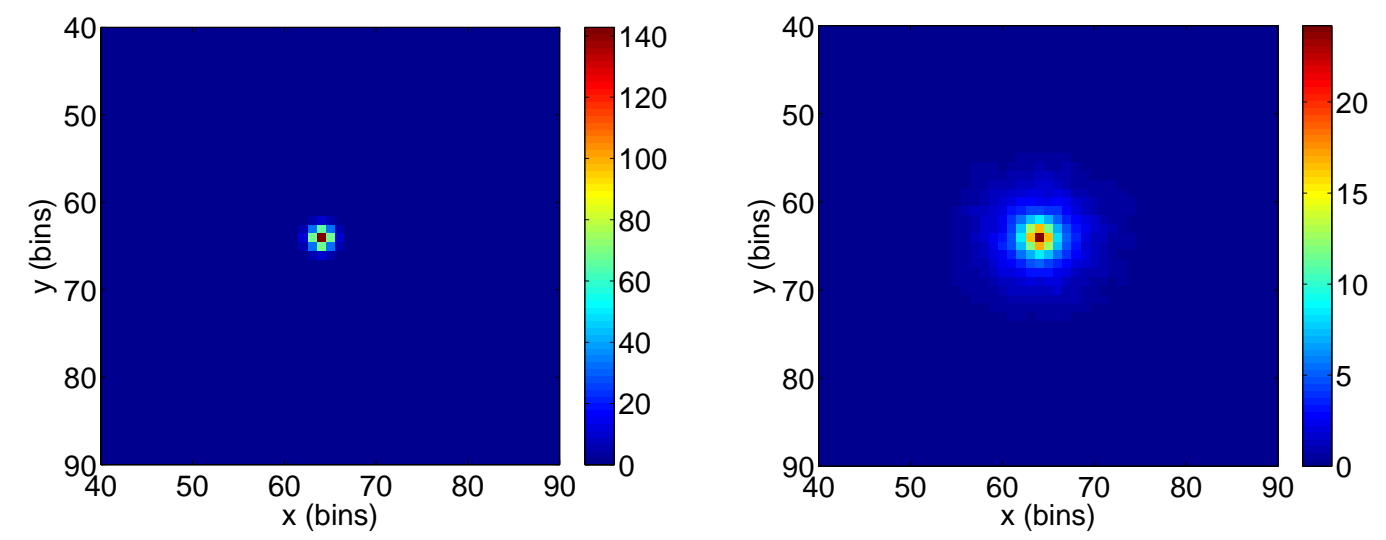

Figure 5.11: Central 3D slice of ${ }^{68} \mathrm{Ga}$ point sources in water at $0 \mathrm{~T}$ with (left) and without (right) the positron range correction for ML-EM iteration 200. 100,000 Poisson distributed events were used and the final reconstructed images where blurred with a 3D $0.7 \mathrm{~mm}$ FWHM Gaussian system resolution.

final reconstructed images where blurred with a 3D $0.7 \mathrm{~mm}$ FWHM Gaussian system resolution. Projections along the x-axis of the images in Figs. 5.11 and 5.13 are shown in Figs. 5.12 and 5.14 for the $0 \mathrm{~T}$ and $7 \mathrm{~T}$ (three magnetic field orientations), respectively. The FWHM and FWTM values for the plots are displayed in Table 5.1. The convergence properties of ${ }^{68} \mathrm{Ga}$ point sources in water for the 3D ML-EM positron range correction algorithm is shown in Figs. 5.15 and 5.16 by plotting the FWHM and FWTM image resolutions versus iteration number. There plot show that the point sources are near full convergence at ML-EM iteration 200.

\subsection{Determining Detector Positions from Calibration Data}

Ideally, the silicon detectors are placed exactly $170 \mathrm{~mm}$ apart, symmetrically on opposite sides of the source. If this is not the case, positioning errors in the end points of the back-projected LORs will occur, degrading the image resolution as a 


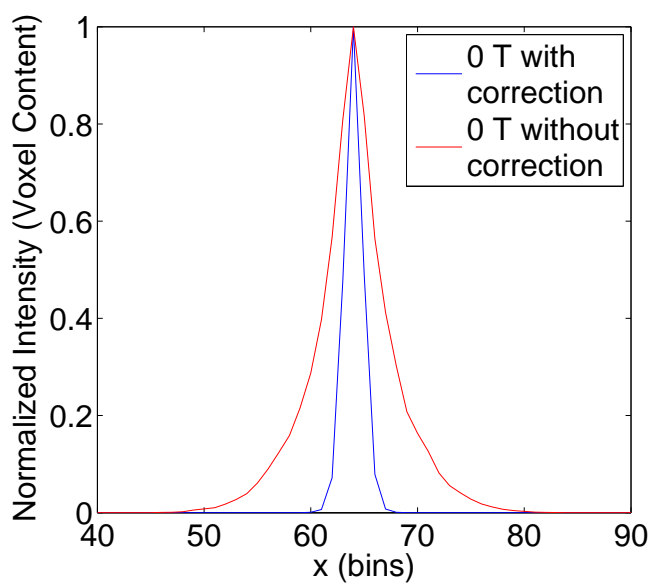

Figure 5.12: Projections along the x-axis of the images shown in Fig. 5.11. The plots are normalized such that the value of the peak in one. The FWHM values are $0.77 \mathrm{~mm}$ and $1.92 \mathrm{~mm}$ for the corrected and the uncorrected data, respectively. The FWTM values are $1.55 \mathrm{~mm}$ and $6.12 \mathrm{~mm}$ for the corrected and uncorrected data, respectively.
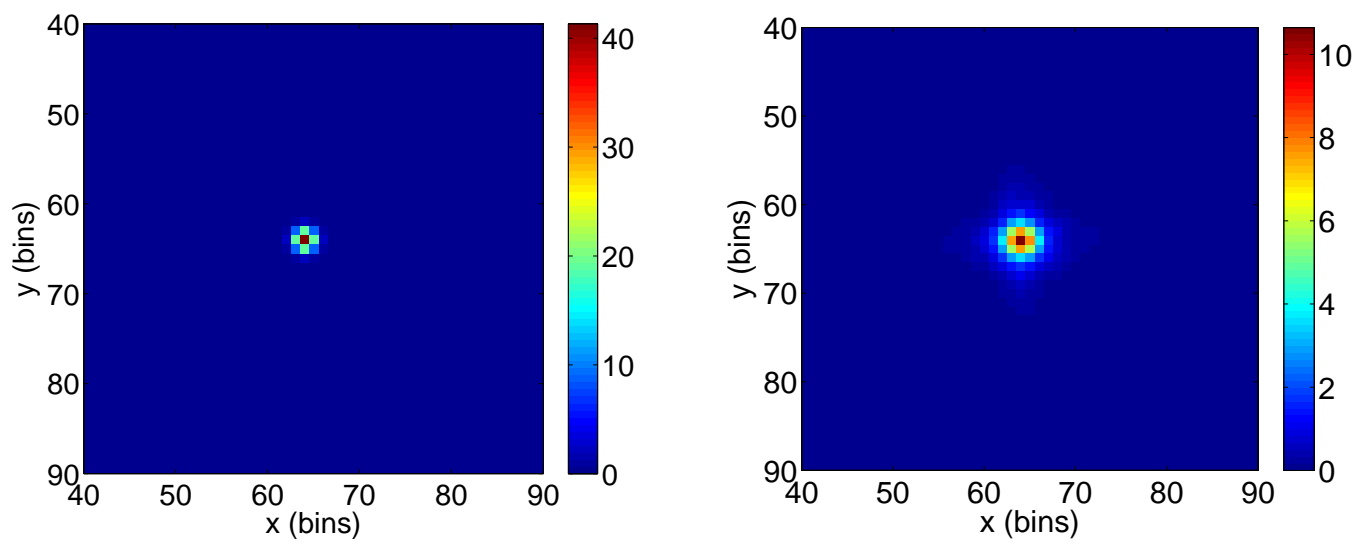

Figure 5.13: Central 3D slice of ${ }^{68} \mathrm{Ga}$ point sources in water at $7 \mathrm{~T}$ with (left) and without (right) the positron range correction for ML-EM iteration 200 using three orthogonal $7 \mathrm{~T}$ data sets simultaneously to reconstruct the images. 100,000 Poisson events were used and the final reconstructed images where blurred with a 3D $0.7 \mathrm{~mm}$ FWHM Gaussian system resolution. 


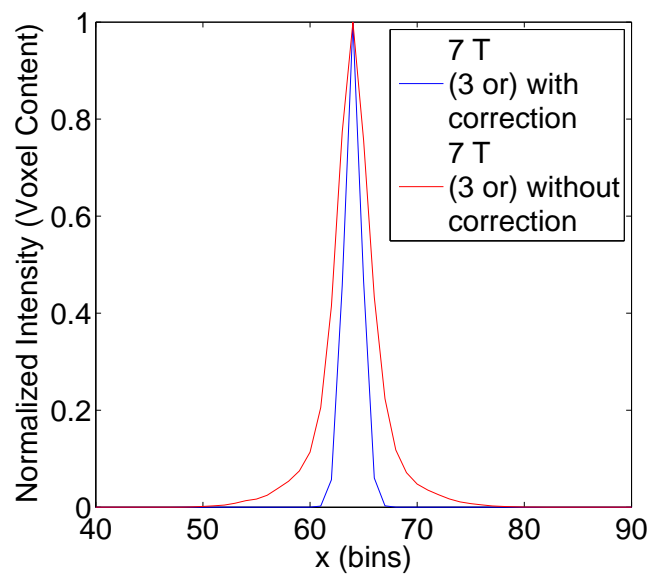

Figure 5.14: Slices along the x-axis through the maxima of the images in Fig. 5.13. The plots are normalized such that the peak values are one. The FWHM values are $0.75 \mathrm{~mm}$ and $1.42 \mathrm{~mm}$ for the corrected and the uncorrected data, respectively. The FWTM values are $1.52 \mathrm{~mm}$ and $3.50 \mathrm{~mm}$ for the corrected and uncorrected data, respectively.

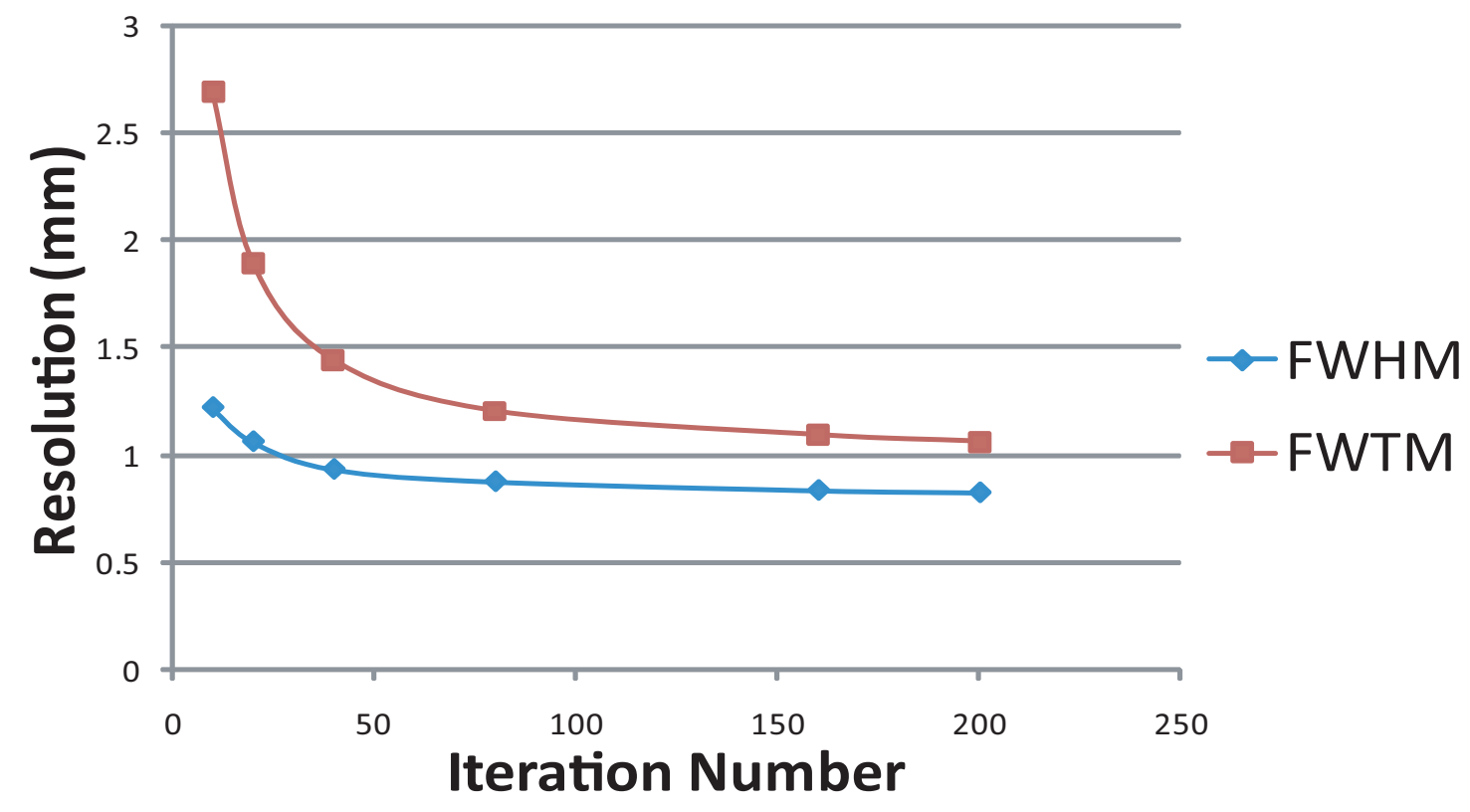

Figure 5.15: FWHM and FWTM resolutions of a ${ }^{68} \mathrm{Ga}$ point source in water at $0 \mathrm{~T}$ versus iteration number using the ML-EM 3D positron range correction algorithm. The image has been blurred for a $0.7 \mathrm{~mm}$ Gaussian system resolution. 


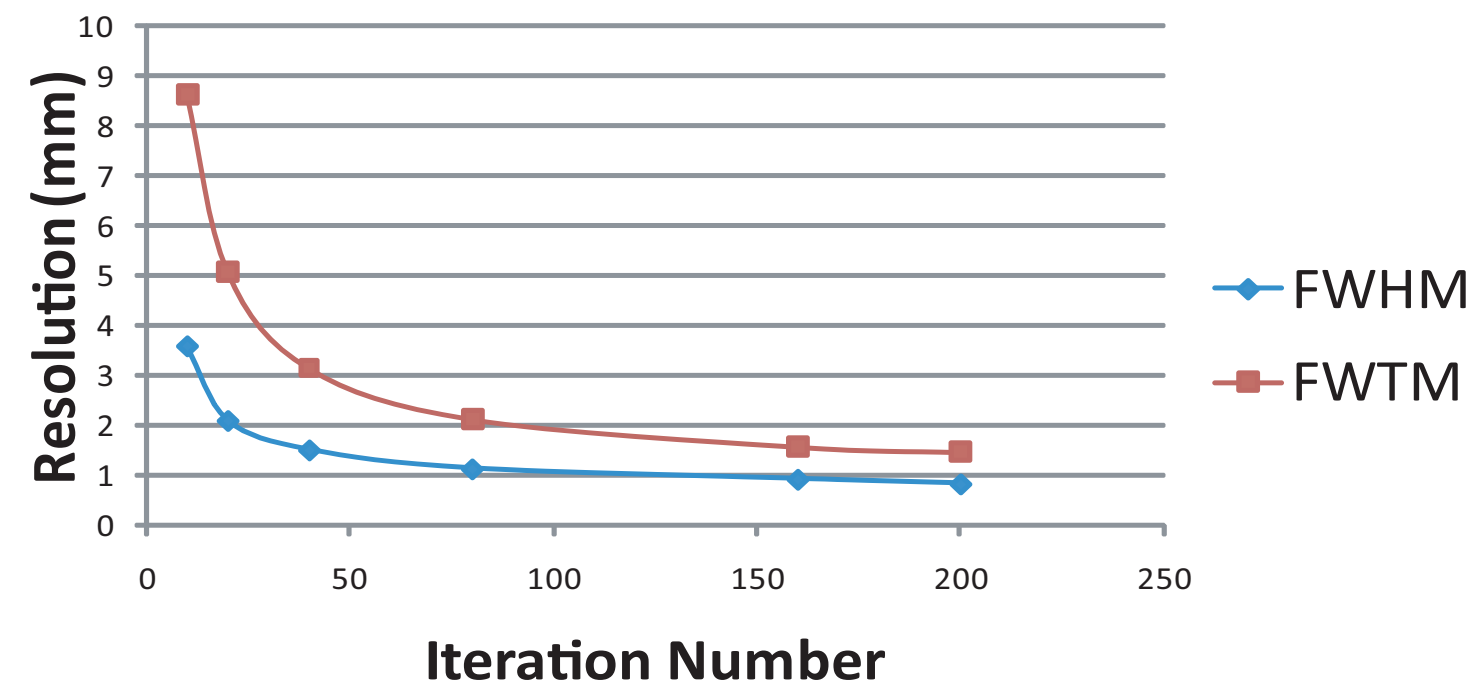

Figure 5.16: FWHM and FWTM resolutions of a ${ }^{68} \mathrm{Ga}$ point source in water at $7 \mathrm{~T}$ versus iteration number using the ML-EM 3D positron range correction algorithm reconstructed with three orthogonal data sets with the positron range distribution from $\mathrm{x}, \mathrm{y}$, and $\mathrm{z}$ magnetic field orientations. The image has been blurred for a $0.7 \mathrm{~mm}$ Gaussian system resolution.

\begin{tabular}{c|cccc} 
Magnetic field & $\begin{array}{c}\text { FWHM }(\mathrm{mm}) \\
\text { (corrected) }\end{array}$ & $\begin{array}{c}\text { FWHM (mm) } \\
\text { (uncorrected) }\end{array}$ & $\begin{array}{c}\text { FWTM (mm) } \\
\text { (corrected) }\end{array}$ & $\begin{array}{c}\text { FWTM (mm) } \\
\text { (uncorrected) }\end{array}$ \\
\hline $0 \mathrm{~T}$ & 0.77 & 1.55 & 1.92 & 6.12 \\
$7 \mathrm{~T}$ & 0.75 & 1.42 & 1.52 & 3.50
\end{tabular}

Table 5.1: FWHM and FWTM values for projections onto the $\mathrm{x}$-axis of a ${ }^{68} \mathrm{Ga}$ point source in water using the 3D ML-EM reconstructed images with and without the positron range correction (ML-EM iteration 200). Both distributions have been blurred by a $0.7 \mathrm{~mm}$ FWHM Gaussian system resolution. 
result. In practice, it is very difficult to position the silicon detector modules with such precision; therefore, it is necessary to have an algorithm that will determine the positioning of the silicon detectors with respect to the center of the field of view using measured calibration data. This section describes the algorithm used to determine the most likely detector positions given a set of measured calibration data.

The form of the input data for the detector position algorithm is a list of the two interaction pads from OSU1 and OSU2. There are 18 such files, each corresponding to a consecutive twenty degree angular increment. Each pad is translated into its corresponding Cartesian coordinate using a look-up table and a random position each pad is selected for the LOR endpoints. The LOR from the two interaction positions is back-projected into the image space, and the closest distance between the LOR and the center of the FOV is calculated. This distance is used in the following function:

$$
F=\sum_{\text {all events }}\left[\log e^{-d^{2}}+e^{-d^{2}}\right]
$$

where $d$ is the distance from the estimated source location to the LOR and the sum is over all collected events. This equation will yield the likelihood that any two detector shifts in $\mathrm{x}$ and $\mathrm{y}$, given by $D 1$ and $D 2$ produce LORs that pass close to the variable source position $S$. Unlike the previously discussed ML-EM algorithms that attempt to maximize the likelihood function, the function presented in equation 5.16 will give the most likely detector geometry when $F$ is minimized. To illustrate this, consider the following two cases in regards to the first term of the equation. Case one, the detectors are positioned in such a way that the LORs pass very close to the source position and consequently the distance between the LORs and the source will approach zero, $d \rightarrow 0$. As $d \rightarrow 0, e^{-d^{2}} \rightarrow 1$, and $-\log e^{d / w} \rightarrow 0$. Thus for detector positions that yield LORs that pass very close to the source position, the 
function $F$ will be a minimum. For the second case, consider detectors positioned such that the LORs are far from the source, $d \rightarrow \infty$. In this situation, $e^{-d^{2}} \rightarrow 0$, and $-\log e^{-d^{2}} \rightarrow \infty$. Therefore, the function $F$ will be large for poor guesses at the detector positions. In order to find the most likely position of the detectors, the MATLAB, fminsearch function is used. There are six variables that the fminsearch function varies to find the minimum value of $F$ : the $\mathrm{x}$ and $\mathrm{y}$ value of the two silicon detectors and the $\mathrm{x}$ and $\mathrm{y}$ values of the estimate source position. An initial guess of the location of both silicon detectors and the positron source position is important because there may exists local minima that do not correspond to the true detector positions.

A calibration measurement is performed before each data collection run to determine the most likely detector positions. This is accomplished by placing a $0.25 \mathrm{~mm}$ diameter ${ }^{22} \mathrm{Na}$ point source at the central position of the turntable source mount, a known position, and collecting data in 18, 20 degree increments. The calibration data is used to calculate the distances $d$ between the LORs and the source position which is used in equation 5.16. Using the detector position algorithm it was also determined that the central hole of the source mount is actually at a radius of $0.2 \mathrm{~mm}$ from the turntable rotation axis. ${ }^{4}$

Figures 5.17 and 5.18 show the uncorrected and corrected sinogram calibration data, respectively The LORs used to create the uncorrected sinogram are used as inputs to the detector estimation algorithm (this sinogram is not corrected for shifts in the detector positions). On close inspection it can be observed that this figure has

\footnotetext{
${ }^{4}$ The offset of the source mount from the rotational axis of the turntable will not degrade the image resolution. The offset will simply shift the radial position of all the source mount positions by a radial distance of $0.2 \mathrm{~mm}$. It is not crucial that the source mount is perfectly centered on the turntable.
} 


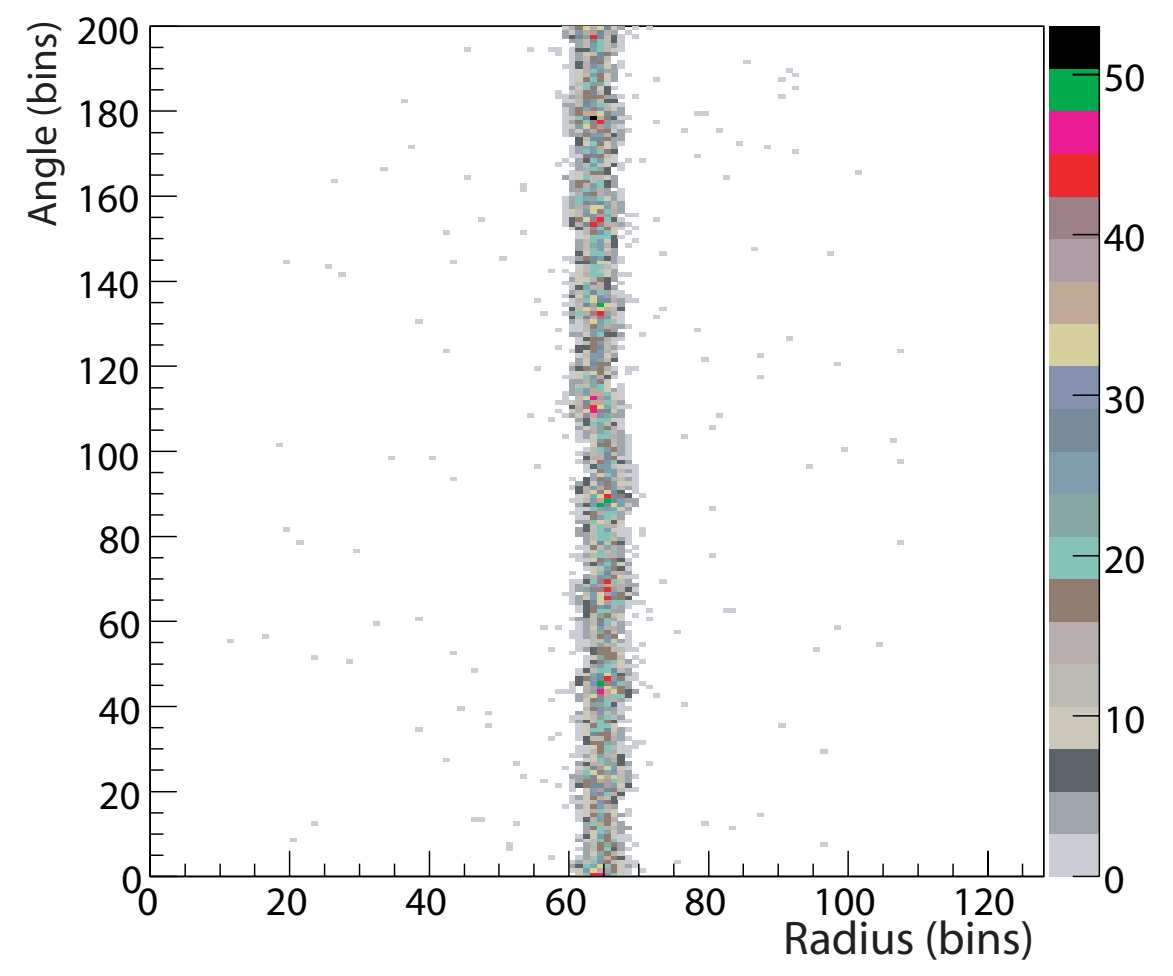

Figure 5.17: The data that was used to create this sinogram is used in the detector estimation algorithm. The sinogram shown here is not corrected for detector shifts.

rough discontinuities along its length. Silicon detector shifts of $(-1.71 \mathrm{~mm}, 0.29 \mathrm{~mm})$ for OSU1 and $(-2.23 \mathrm{~mm}, 0.29 \mathrm{~mm})$ for OSU2 in $\mathrm{x}$ and $\mathrm{y}$ were found using this data. The corrected sinogram in Fig. 5.18 is the same data shown in the sinogram on the left but using the detector shift corrections. The discontinuities in the sinogram are no longer present. Figures 5.17 and 5.18 is the sinogram from data collected from a ${ }^{22} \mathrm{Na}$ point source positioned $6 \mathrm{~mm}$ from the center of the FOV, transverse to a $7 \mathrm{~T}$ magnetic field. The sinogram in Fig. 5.17 is created without the detector shift corrections while the sinogram in Fig. 5.18 uses the detector shift corrections determined from the calibration data. The resolution of the final reconstructed images of the uncorrected and corrected data is $1.0 \mathrm{~mm}$ FWHM and $0.8 \mathrm{~mm}$ FWHM, respectively. 


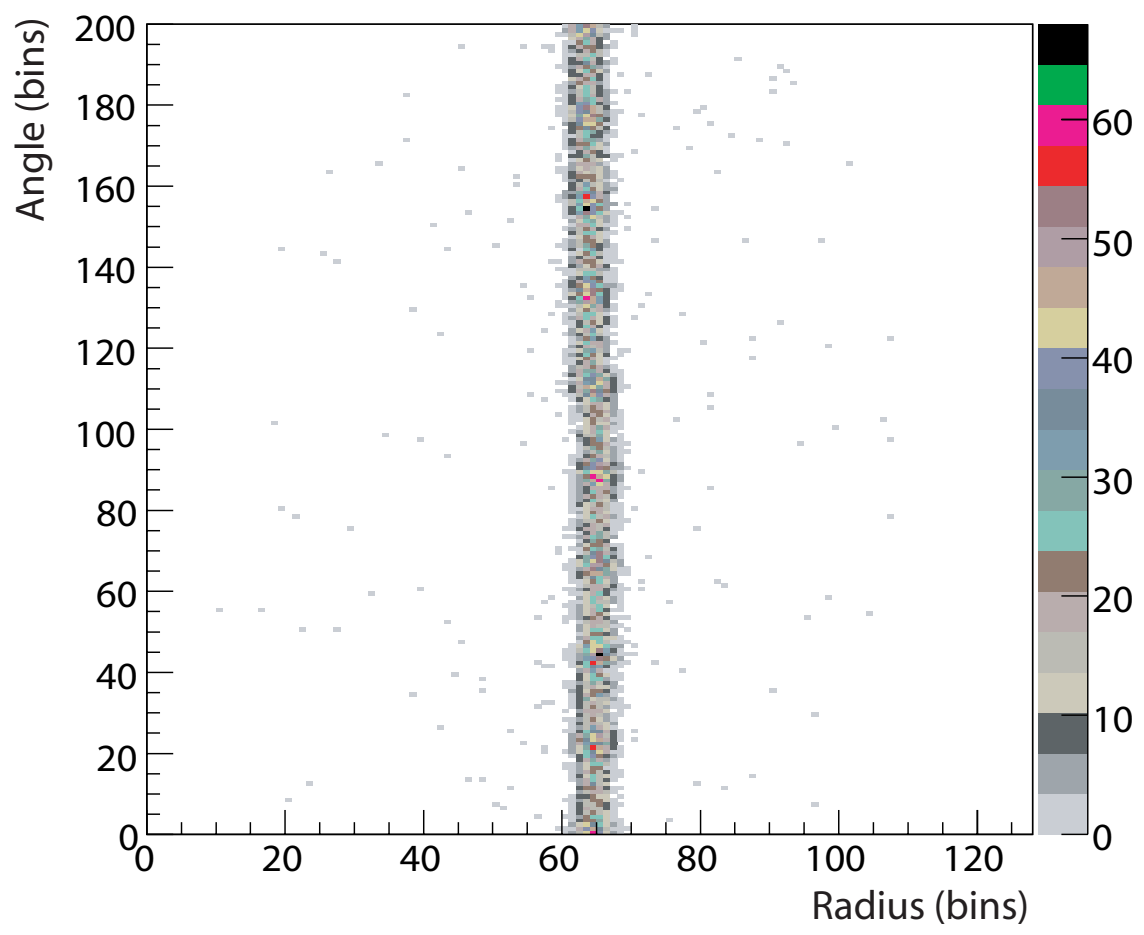

Figure 5.18: This sinogram is created using the same data with the detector shifts determined by the detector estimation algorithm. The shifts in $\mathrm{x}$ and $\mathrm{y}$ are $(-1.71 \mathrm{~mm}$, $0.29 \mathrm{~mm})$ for the first detector and $(-2.23 \mathrm{~mm}, 0.29 \mathrm{~mm})$ for the second detector. The discontinuities in the sinogram are no longer present. 


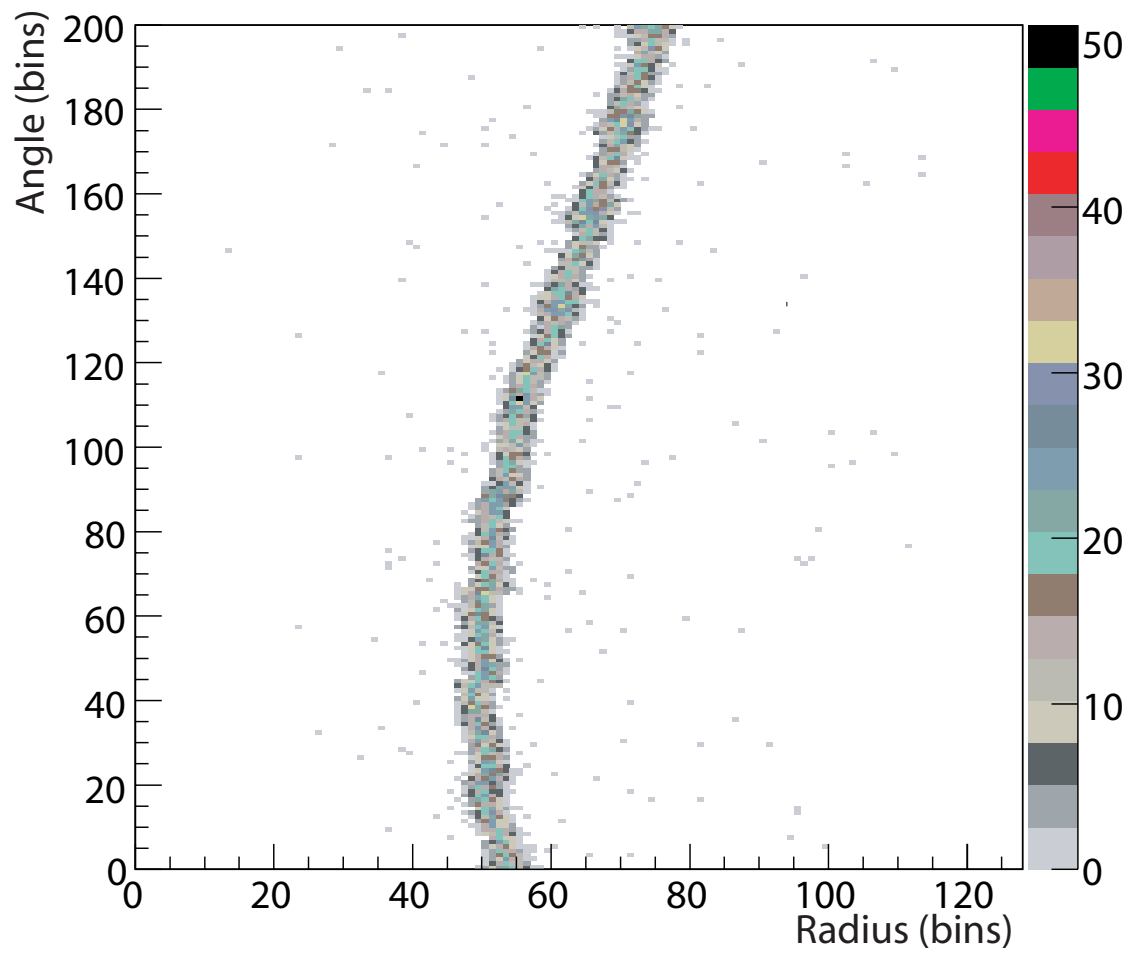

Figure 5.19: This sinogram is created without any detector shift corrections assuming the detectors are positioned perfectly. On close inspection it can be observed that this figure has rough discontinuities along its length. 


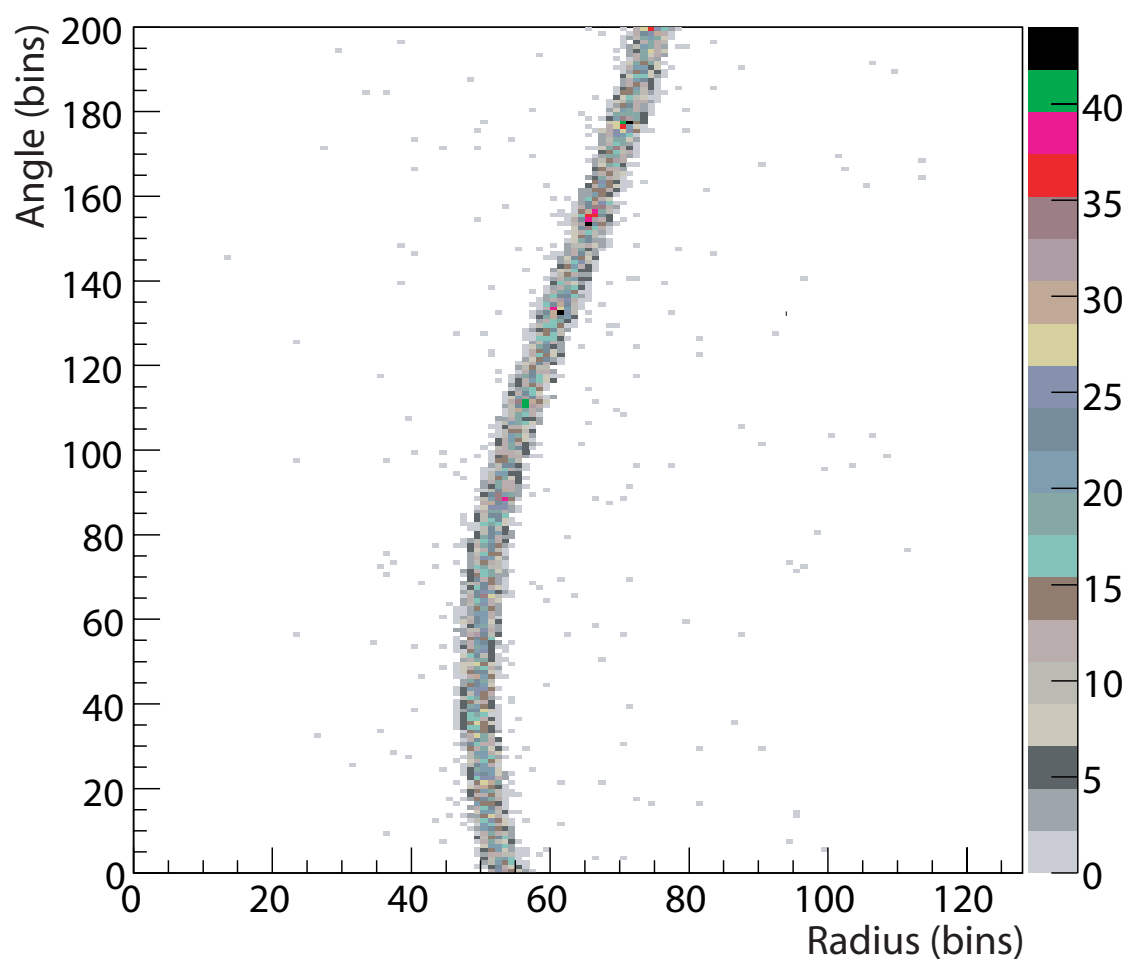

Figure 5.20: This sinogram is created using the same data that was used to create the sinogram in Fig. 5.19, but uses the detector shifts of $(-1.71 \mathrm{~mm}, 0.29 \mathrm{~mm})$ and $(-2.23$ $\mathrm{mm}, 0.29 \mathrm{~mm})$. Note that the discontinuities are no longer present. The difference in the final reconstructed images is $1.0 \mathrm{~mm}$ FWHM using the uncorrected sinogram compared to $0.8 \mathrm{~mm}$ FWHM using the corrected sinogram. 


\section{CHAPTER 6}

\section{Experimental Results}

\subsection{Introduction: Purpose of Chapter}

The purpose of this chapter is to present the ML-EM reconstructed images of the data collected with the small animal test bench described in chapter 4 for a low kinetic energy positron emitter $\left({ }^{22} \mathrm{Na}\right)$ and a high kinetic energy positron emitter $\left({ }^{68} \mathrm{Ga}\right)$ in water equivalent plastic at both $0 \mathrm{~T}$ an in a $7 \mathrm{~T}$ magnetic field directed transverse to the image plane. The experimental results are also compared to the simulation. This chapter also presents the results of artifacts that can occur for positron emitters positioned off-plane from the current detector plane for both $0 \mathrm{~T}$ and with a $7 \mathrm{~T}$ magnetic field applied transverse to the image plane.

\subsection{Expected Image Resolution}

A previous small animal PET system was constructed at the University of Michigan using the same silicon detectors in an analogous set-up [7] [44]. In that analysis, the image resolution of the system was measured using a low energy positron emitter ${ }^{18} \mathrm{~F}$ contained in a $0.254 \mathrm{~mm}$ inner diameter steel syringe. The outer, steel wall of the syringe was $0.127 \mathrm{~mm}$ thick. The $1.0 \mathrm{~mm}$ plane defined by the silicon detectors was 
collimated by lead shielding to reduce the background rate from off-axis annihilations that would otherwise saturate the system. The natural ${ }^{18} \mathrm{~F}$ positron range contribution to the resolution is nearly eliminated by the steel; however, contributions to the final image resolution due to the size of the source must still be accounted for when determining the intrinsic system resolution. The activity in the collimated slit was estimated to be $1.0 \mu \mathrm{Ci}$. The image resolution was measured to be $0.77 \mathrm{~mm}$ by a projection through the center of the source. After a deconvolution of the source size, a $0.70 \mathrm{~mm}$ intrinsic detector resolution was estimated [44].

The previous experimental results are confirmed using the EGS4 simulation. In this simulation, the geometry described in the previous paragraph is modeled. Fig. 6.1 shows a density histogram of the positions of the positron-electron annihilations in the detector plane (left). The image on the right in Fig. ?? is a projection of the positron-electron annihilation points projected onto the x-axis.
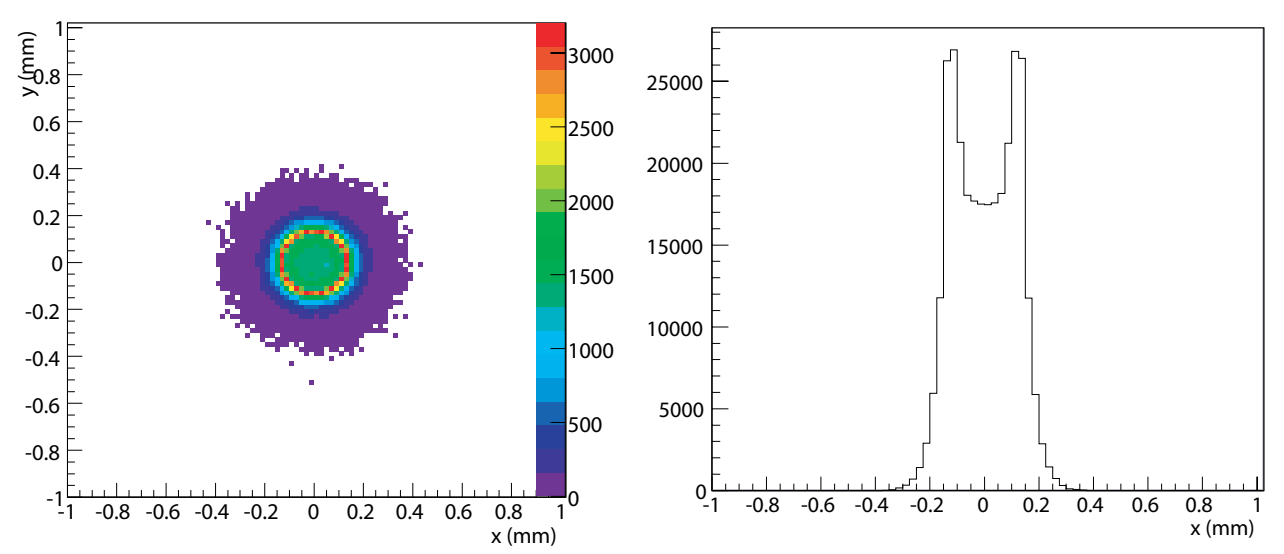

Figure 6.1: Left: two dimensional density histogram of the positron-electron annihilation positions in the detector plane of a ${ }^{18} \mathrm{~F} 0.254 \mathrm{~mm}$ diameter liquid source contained within a $0.127 \mathrm{~mm}$ thick steel syringe. Right: The projection of Fig. 6.1 onto the x-axis. 


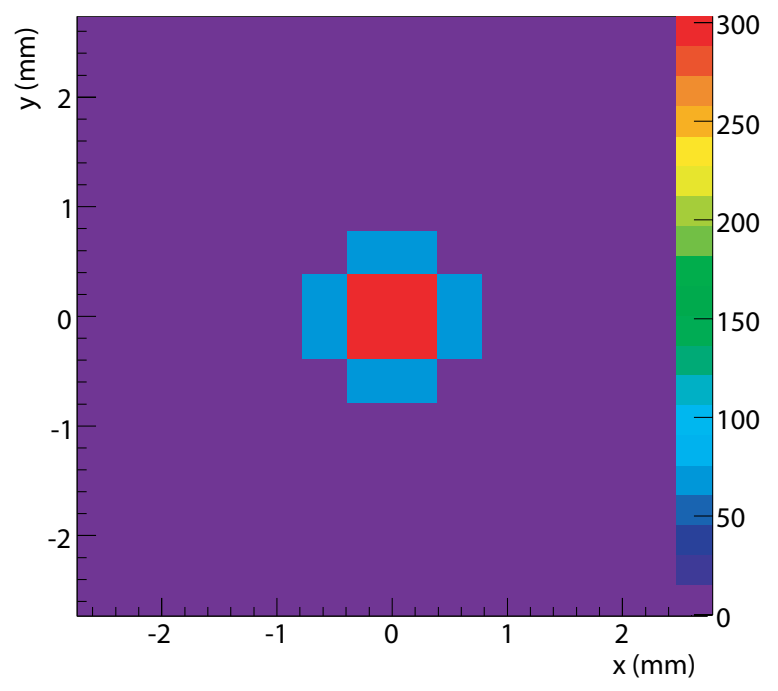

Figure 6.2: ML-EM reconstruct image (iteration 20) of the EGS4 simulated ${ }^{18} \mathrm{~F}$ source contained in a $0.254 \mathrm{~mm}$ inner diameter steel syringe.

The positron-electron annihilation positions, along with the random photon accollinearity angle are used in the second half of the EGS4 simulation that tracks the positrons through the silicon detectors. The simulated data is collected in eighteen, twenty degree increments. The results of this, full EGS4 simulation are then reconstructed with the ML-EM reconstruction algorithm, terminated at the twentieth iteration. Fig. 6.2 is the final reconstructed image of a liquid ${ }^{18} \mathrm{~F}$ source contained in a steel syringe, and Fig. 6.3 is the x-projection of the reconstructed image. Fig. 6.2 is very pixellated because this is a zoomed in view of the source. The source is located at the junction of four, $0.4 \mathrm{~mm} \times 0.4 \mathrm{~mm}$ pixels. The x-projection is fit to a Gaussian function with a width of $0.73 \mathrm{~mm}$ FWHM. This simulation result is consistent with the previously measured value of the image resolution. 


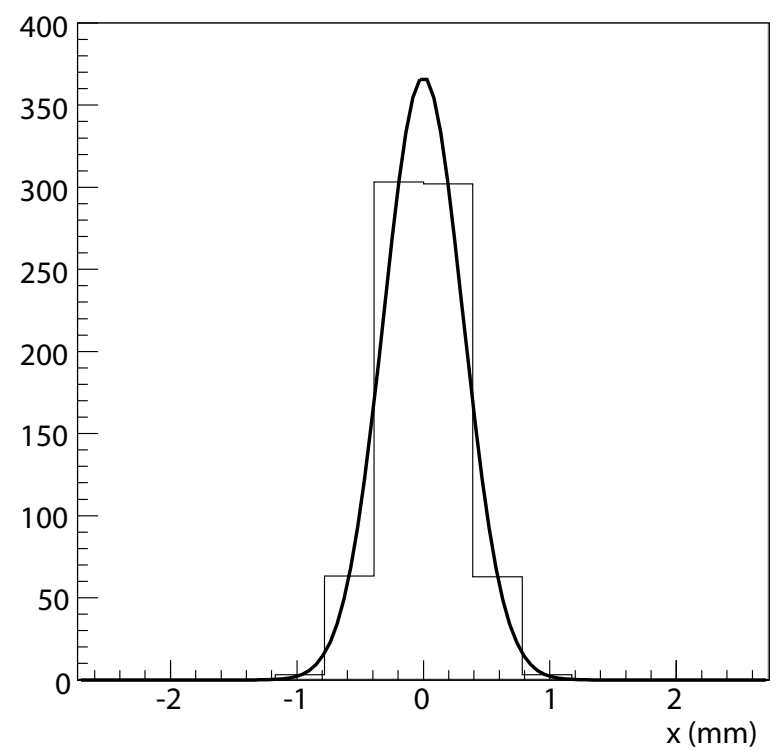

Figure 6.3: The x-projection of the image shown in Fig. 6.2

\subsection{Comparison of Measured and Simulated Results}

In order to confirm that our intrinsic image resolution is comparable to the previous set-up, a simple quadrature estimate of the image resolution is performed on the $0 \mathrm{~T}^{22} \mathrm{Na}$ measurement to determine the system resolution.

Two point sources were chosen to test the small animal PET test bench, a low energy positron emitter, ${ }^{22} \mathrm{Na}$, and a high energy positron emitter, ${ }^{68} \mathrm{Ga} .{ }^{68} \mathrm{Ge}$ (half life 275 days) was used as a stable source of the high energy positron emitting ${ }^{68} \mathrm{Ga}$ radioisotope (half life 68 minutes). ${ }^{68} \mathrm{Ge}$ decays into ${ }^{68} \mathrm{Ga}$ following an electroncapture process [52]. Both sources are enclosed in the center of a cylinder of water equivalent plastic, $1 / 4$ inch thick and one inch in diameter produced by Eckert \& Ziegler Isotope Products (Fig. 6.4). The ${ }^{22} \mathrm{Na}$ and ${ }^{68} \mathrm{Ga}$ point sources have diameters of $0.25 \mathrm{~mm}$ and $1.0 \mathrm{~mm}$, respectively. 


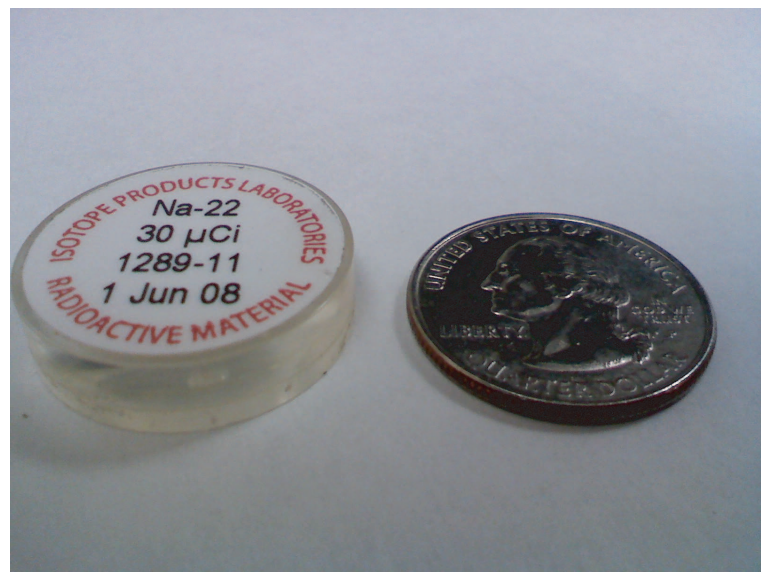

Figure 6.4: Positron emitting sources embedded in a water equivalent plastic of density $1.08 \mathrm{~g} / \mathrm{cm}^{3}$ manufactured by Eckert \& Ziegler Isotope Products [53].

Coincidence data was collected in 20 degree angular increments in order to obtain a data set with full angular sampling. The images were reconstructed using the previously described ML-EM algorithm incorporating the geometric system matrix describing our experimental set-up but with no accounting for positron range [7]. The ML-EM algorithm was terminated after twenty iterations in all of the following images in order to avoid introducing statistical noise. The magnetic field for the $7 \mathrm{~T}$ measurements was oriented transverse to the plane of the images.

Figures 6.5 through 6.11 contain the ML-EM reconstructed images of the measured data for both the ${ }^{22} \mathrm{Na}$ and ${ }^{68} \mathrm{Ga}$ point sources. In these figures, the left and center images are the reconstruction of the measured and simulated data, respectively. The image on the right is a comparison between the reconstructed measured and simulated images projected onto the $\mathrm{x}$-axis.

The results for the ${ }^{22} \mathrm{Na}$ point source are displaced in both Fig. 6.5 for $0 \mathrm{~T}$ and in Fig. 6.6 for $7 \mathrm{~T}$. The x-projections of the reconstructed ${ }^{22} \mathrm{Na}$ distributions were 

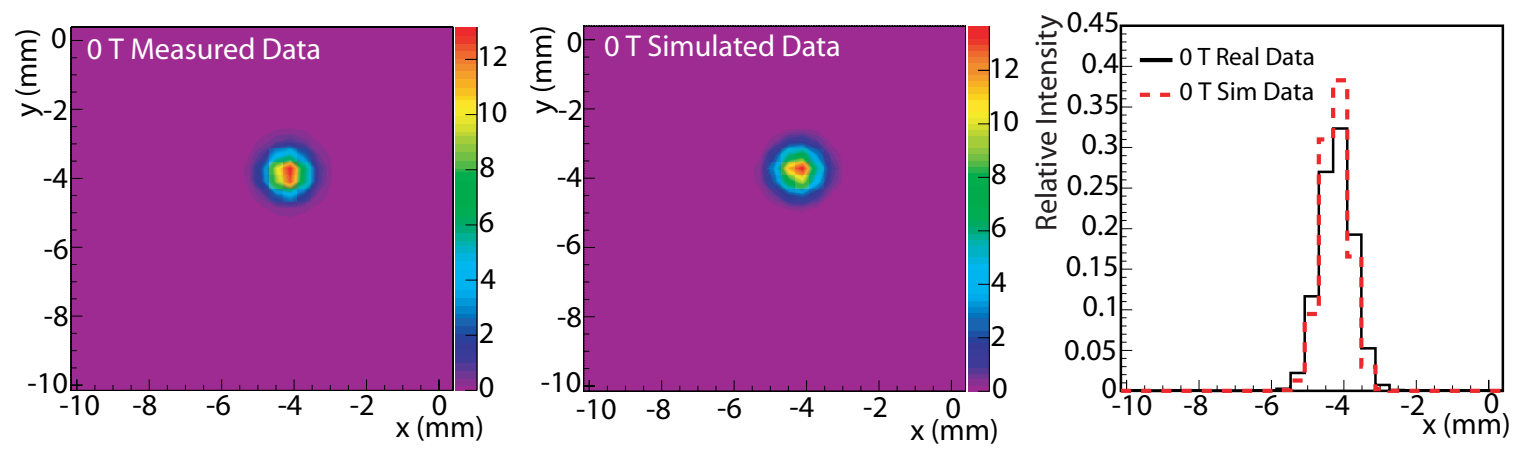

Figure 6.5: ML-EM reconstructed image of measured data (left image) and simulated data (middle image) of a $0.25 \mathrm{~mm}$ diameter ${ }^{22} \mathrm{Na}$ point source encased in a water equivalent plastic at $0 \mathrm{~T}$. The image on the right is the comparison of the measured and simulated data projected onto the $\mathrm{x}$-axis.
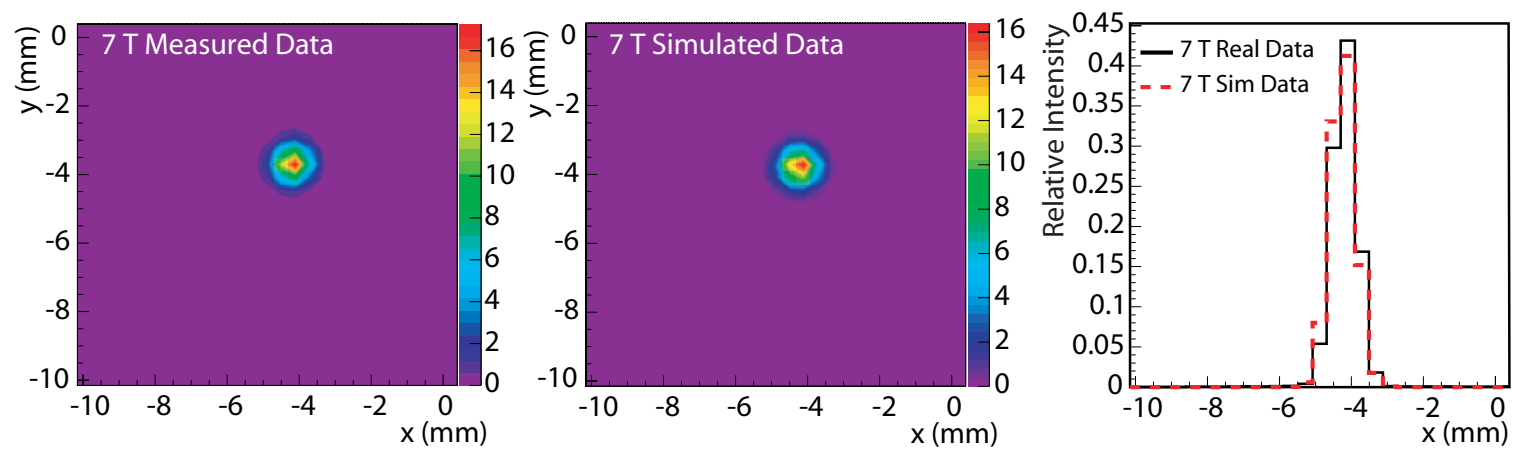

Figure 6.6: ML-EM reconstructed image of measured data (left image) and simulated data (middle image) of a $0.25 \mathrm{~mm}$ diameter ${ }^{22} \mathrm{Na}$ point source encased in a water equivalent plastic with a $7 \mathrm{~T}$ magnetic field applied transverse to the plane of the image. The image on the right is the comparison of the measured and simulated data projected onto the $\mathrm{x}$-axis. 
fit to a Gaussian, and the FWHM and FWTM were extracted. The FWHM and FWTM of the projection of the images onto the x-axis is listed in Table 6.1 for $0 \mathrm{~T}$ and $7 \mathrm{~T}$. These results confirm the simulation results that strong magnetic fields will not markedly improve the resolution of low energy positron emitters such as ${ }^{22} \mathrm{Na}$ and ${ }^{18} \mathrm{~F}$ in water.

It is now possible to answer the question: what is the intrinsic image resolution of our system? The resolution of any PET system can be estimated using [54]

$$
\Gamma=\sqrt{r^{2}+(0.0025 D)^{2}+(2 R)^{2}+\sigma^{2}}
$$

where $r$ is the rms positron range, $D$ is the inner diameter of the PET scanner which is used to estimates the uncertainty due to photon acollinearity, $R$ is the radius of the source, and $\sigma$ is the intrinsic system resolution. Solving equation 6.1 for $\sigma$, and using $\Gamma=0.90 \mathrm{~mm}$ (the FWHM measured ${ }^{22} \mathrm{Na}$ resolution),$r=0.25 \mathrm{~mm}$ (from the simulation results in section 2.7$), D=170 \mathrm{~mm}$, and $R=0.25 \mathrm{~mm}$ yields a resolution of $\sigma=0.72 \mathrm{~mm}$ which is in excellent agreement with the expected system resolution of $0.7 \mathrm{~mm}$ FWHM.

Figures 6.7 and 6.8 contain the measured and simulated results of two $0.25 \mathrm{~mm}$ diameter ${ }^{22} \mathrm{Na}$ point sources separated by $1.5 \mathrm{~mm}$ from their centers. The measurements and simulations are in good agreement. Note that the $0 \mathrm{~T}$ measurement of the dual point sources was collected at a slight angle with respect to a line normal to the inner edge of the silicon detectors. This angle is small enough, and the binning course enough, that the projection onto the $\mathrm{x}$-axis is still a reasonable representation of the system response. Fig 6.9 shows the comparison between the dual point measured and simulation projections at $0 \mathrm{~T}$ and $7 \mathrm{~T}$ for the measured data (left) and the simulated data (right). 

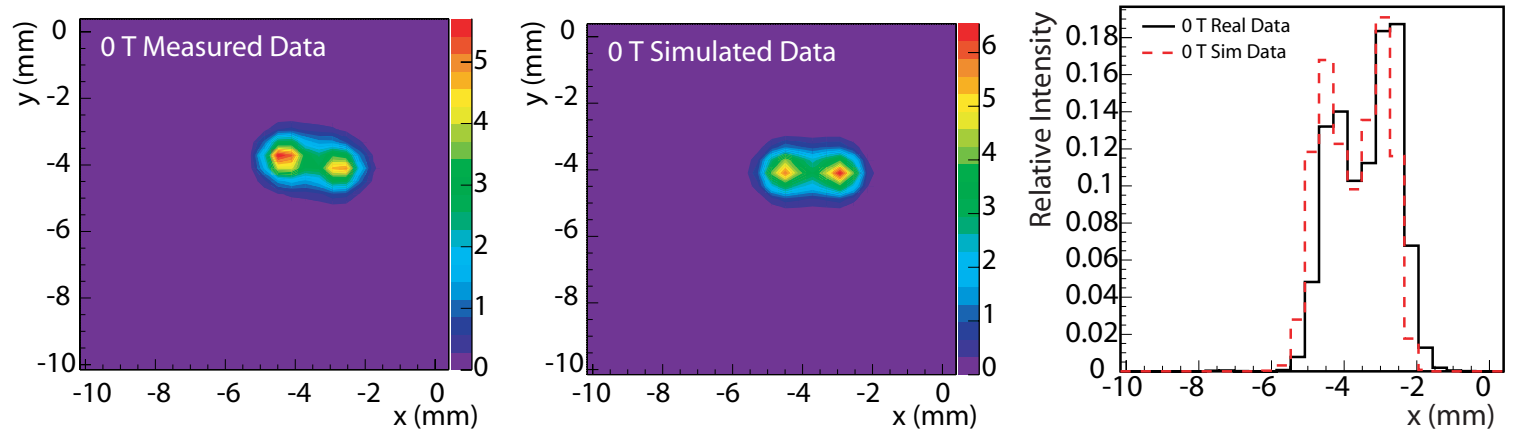

Figure 6.7: ML-EM reconstructed image of measured data (left image) and simulated data (middle image) of two $0.25 \mathrm{~mm}$ diameter ${ }^{22} \mathrm{Na}$ point source separated by $1.5 \mathrm{~mm}$ from their centers. The sources are encased in a water equivalent plastic at $0 \mathrm{~T}$. The image on the right is the comparison of the measured and simulated data projected onto the x-axis.
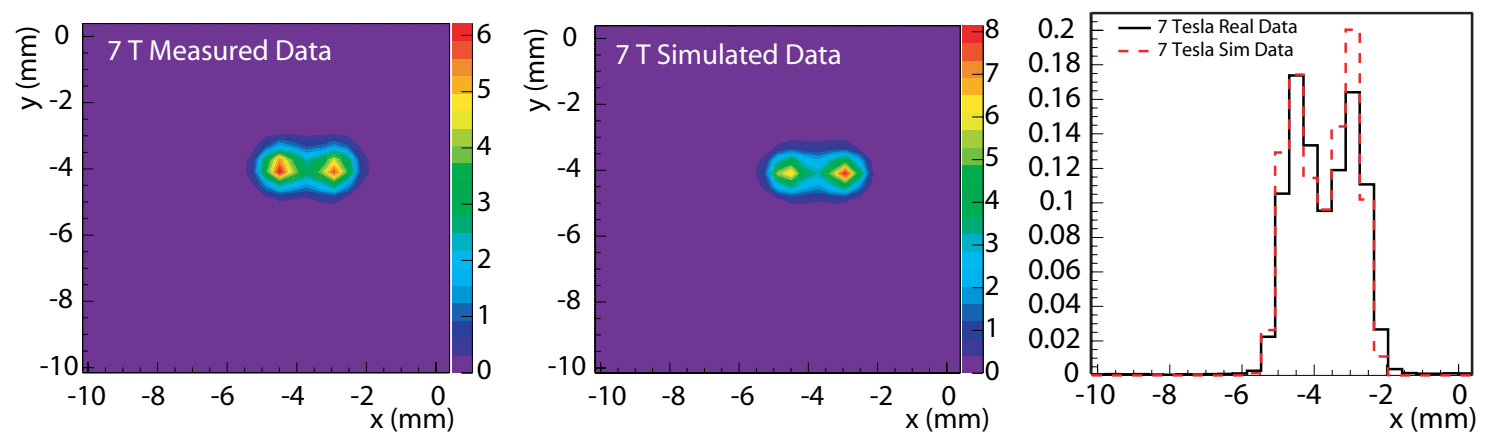

Figure 6.8: ML-EM reconstructed image of measured data (left image) and simulated data (middle image) of two $0.25 \mathrm{~mm}$ diameter ${ }^{22} \mathrm{Na}$ point source separated by $1.5 \mathrm{~mm}$ from their centers. The sources are encased in a water equivalent plastic at $7 \mathrm{~T}$. The image on the right is the comparison of the measured and simulated data projected onto the x-axis. 

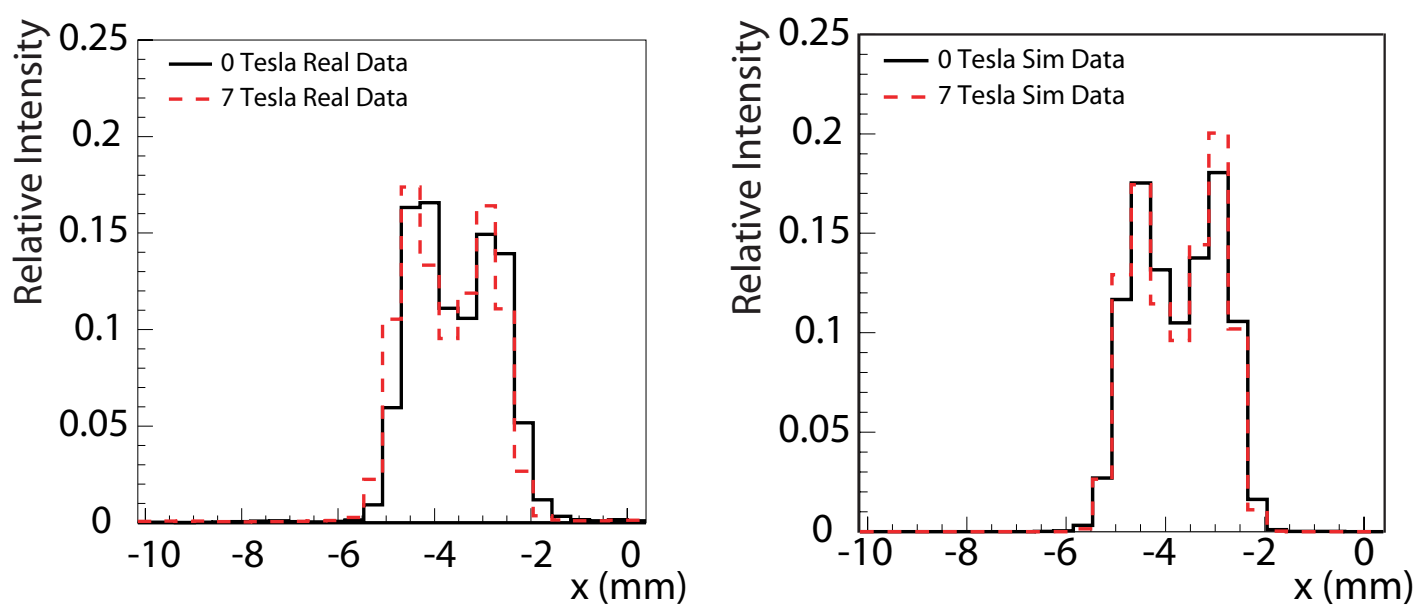

Figure 6.9: Comparisons of the measured (left) and simulated (right) x-projections of the dual ${ }^{22} \mathrm{Na}$ study from figures 6.7 and 6.8
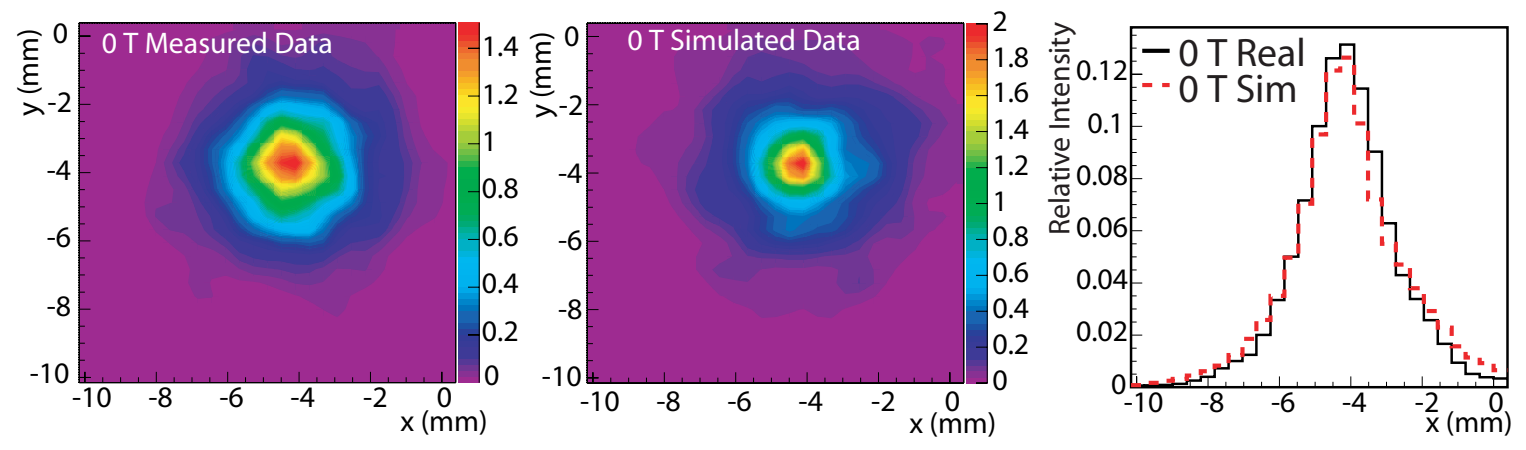

Figure 6.10: ML-EM reconstructed image of measured data (left image) and simulated data (middle image) of a $1.0 \mathrm{~mm}$ diameter ${ }^{68} \mathrm{Ga}$ point source encased in a water equivalent plastic at $0 \mathrm{~T}$. The image on the right is the comparison of the measured and simulated data projected onto the $\mathrm{x}$-axis. 

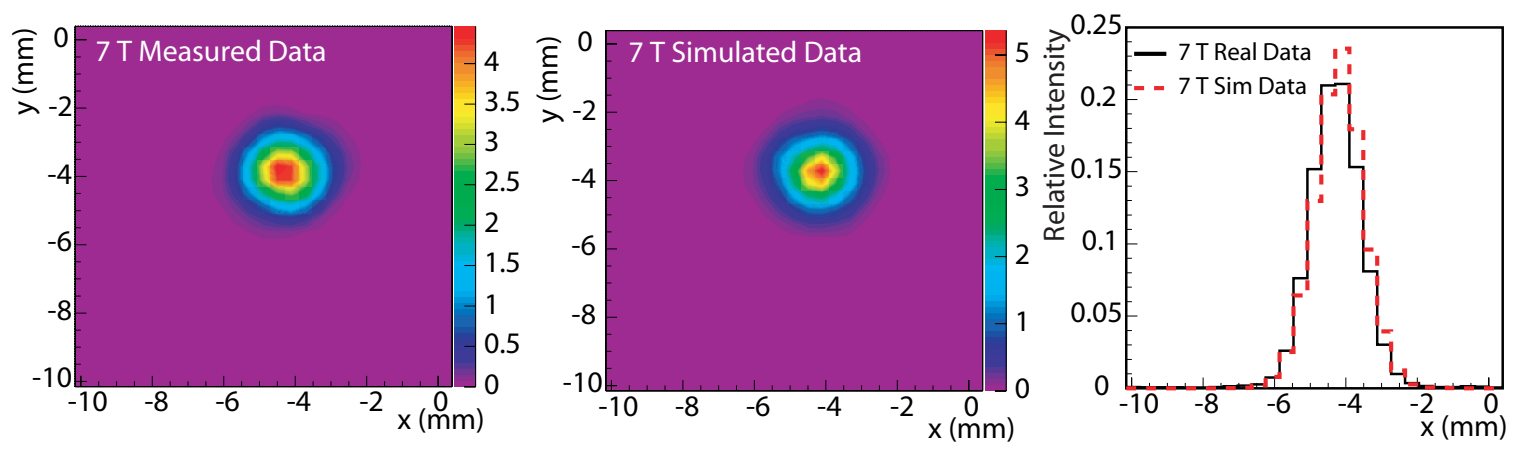

Figure 6.11: ML-EM reconstructed image of measured data (left image) and simulated data (middle image) data of $1.0 \mathrm{~mm}$ diameter ${ }^{68} \mathrm{Ga}$ point source encased in a water equivalent plastic with a $7 \mathrm{~T}$ magnetic field applied transverse to the image plane. The image on the right is the comparison of the measured and simulated data projected onto the x-axis.

The results for the ${ }^{68} \mathrm{Ga}$ point source are displayed in both Fig. 6.10 for $0 \mathrm{~T}$ and in Fig. 6.11 for $7 \mathrm{~T}$. The $\mathrm{x}$-projections of the reconstructed ${ }^{68} \mathrm{Ga}$ distributions were fit to a double Gaussian function for the $0 \mathrm{~T}$ data and a Gaussian function for the $7 \mathrm{~T}$ data. The FWHM and FWTM values listed in Table 6.1 were extracted from these fits. These measurements confirm the simulation results that strong magnetic fields will greatly reduce the tails of the positron annihilation distribution for high energy positron emitters and lead to higher resolution images.

In order to better demonstrate the benefits of placing high energy positron emitting sources in strong magnetic fields, the following source configuration was tested. Two $1.0 \mathrm{~mm}$ diameter ${ }^{68} \mathrm{Ga}$ point sources were positioned $3.6 \mathrm{~mm}$ apart with respect to the center of their distributions. The source on the right is a factor of ten times more active than the source on the left. Fig. 6.12 contains the $0 \mathrm{~T}$ and $7 \mathrm{~T}$ ML-EM reconstructed images of the dual point configuration. At $0 \mathrm{~T}$ the lower activity point 

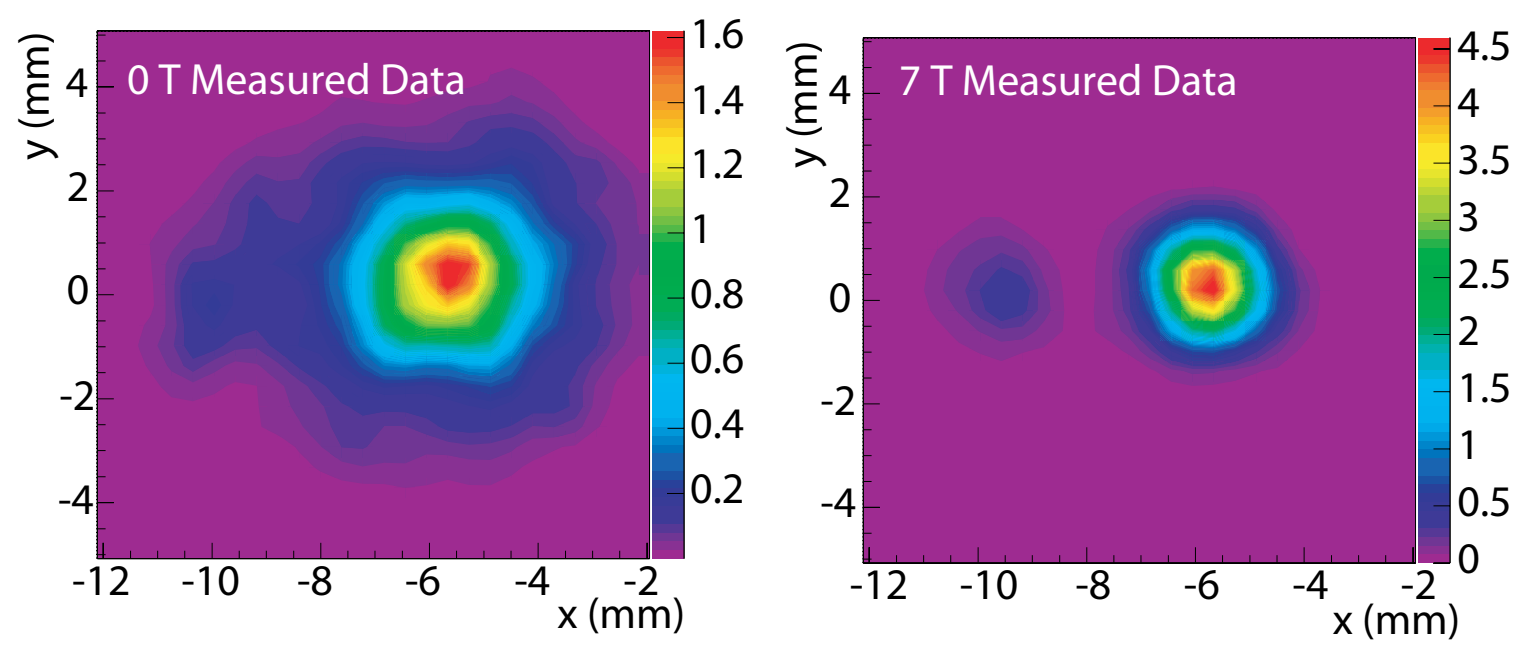

Figure 6.12: ML-EM image of two $1.0 \mathrm{~mm}$ diameter ${ }^{68} \mathrm{Ga}$ point sources separated by $3.6 \mathrm{~mm}$ from their centers at $0 \mathrm{~T}$ (left) and in a $7 \mathrm{~T}$ (right) magnetic field directed transverse to the image plane. The source on the right possess a factor of 10 higher activity than the source on the left. Both of the image are reconstructed from measured data.

source can not be resolved; however, the lower activity point source is easily distinguished at $7 \mathrm{~T}$. The projections of the previous images along the $\mathrm{x}$-axis are shown in Fig. 6.13 for $0 \mathrm{~T}$ and $7 \mathrm{~T}$, respectively. The projections of the simulated reconstructed EGS4 data are plotted in the images as well. The measured and simulated data are in good agreement for both $0 \mathrm{~T}$ and $7 \mathrm{~T}$. This dual point study illustrates the importance of reducing the tails of higher energy positron emitters for resolving lower activity sources in the presence of higher activity sources

\subsection{Comparison to Other Results}

Previous measurements of both ${ }^{22} \mathrm{Na}$ and ${ }^{68} \mathrm{Ga}$ point sources were performed by Hammer and Christensen [55] [56]. Their experimental set-up consisted of two CsI(Tl) 

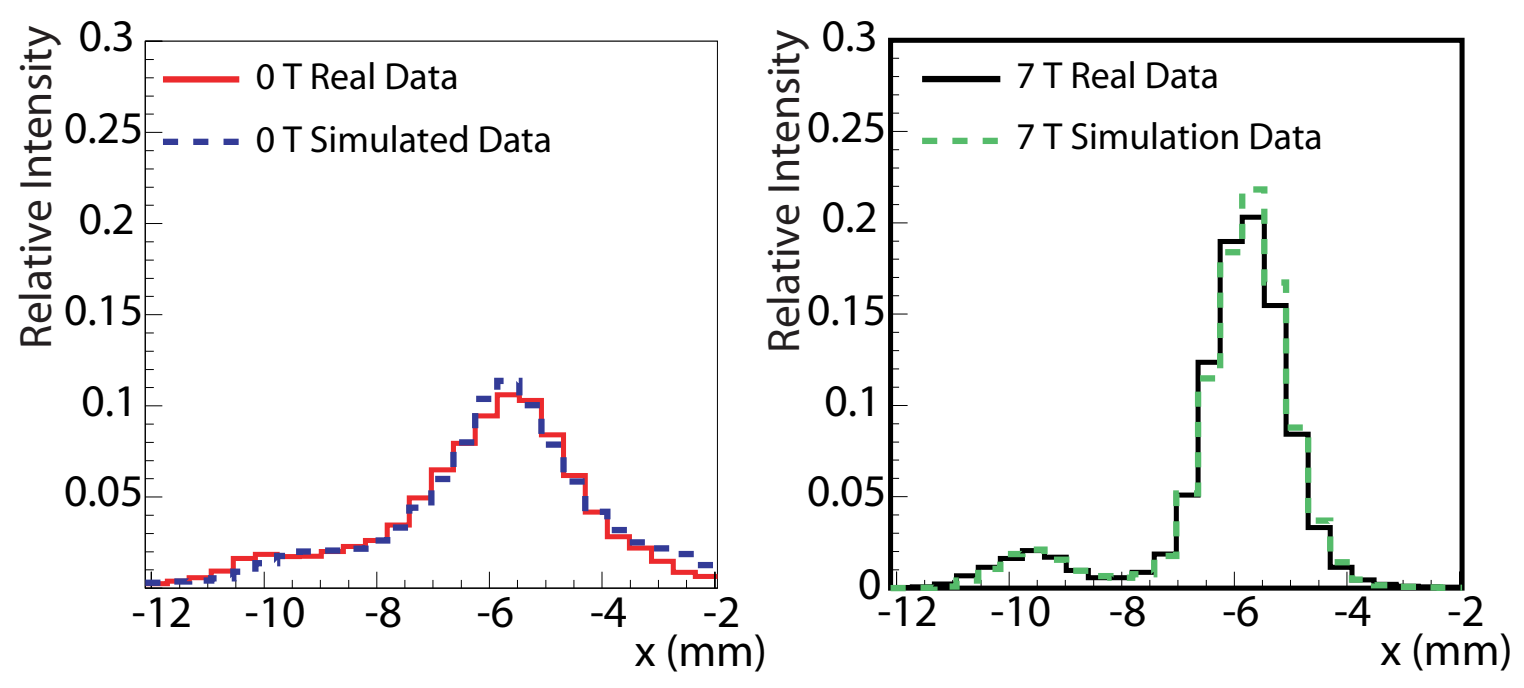

Figure 6.13: The x-projections of two $1.0 \mathrm{~mm}$ diameter ${ }^{68} \mathrm{Ga}$ point sources separated by a $3.6 \mathrm{~mm}$ from their centers at 0 Tesla (left) and in a $7 \mathrm{~T}$ magnetic field directed transverse to the image plane (right). The source on the left possesses 10 times the activity as the source on the left.

scintillation crystals coupled to photodiodes separated by $164 \mathrm{~mm}$. Their $0.500 \mathrm{~mm}$ diameter positron source was positioned at the center of the field of view, and the annihilation photons were collimated into either a $0.2 \mathrm{~mm}$ x $25 \mathrm{~mm}$ slit or $1.0 \mathrm{~mm}$ x $25 \mathrm{~mm}$ slit. The source was moved across the detection volume defined by the slits, and the number of coincidence counts was recorded for each position. The resulting data of coincidence counts per unit time yields the point spread function of the positron range for each source. Tables 6.2 and 6.3 compare the results of Hammer and Christensen and the results from the very high resolution small animal PET for ${ }^{22} \mathrm{Na}$ and ${ }^{68} \mathrm{Ga}$ point sources, respectively. 


\begin{tabular}{lcccc} 
& \multicolumn{2}{c}{${ }^{22} \mathrm{Na}$} & \multicolumn{2}{c}{${ }^{68} \mathrm{Ga}$} \\
\hline & FWHM $(\mathrm{mm})$ & FWTM $(\mathrm{mm})$ & FWHM $(\mathrm{mm})$ & FWTM $(\mathrm{mm})$ \\
$0 \mathrm{~T}$ (measured) & $0.90 \pm 0.08$ & $1.65 \pm 0.14$ & $2.30 \pm 0.12$ & $5.70 \pm 0.29$ \\
$0 \mathrm{~T}$ (simulated) & $0.94 \pm 0.08$ & $1.72 \pm 0.15$ & $2.27 \pm 0.12$ & $6.60 \pm 0.32$ \\
$7 \mathrm{~T}$ (measured) & $0.81 \pm 0.07$ & $1.49 \pm 0.11$ & $1.60 \pm 0.11$ & $3.10 \pm 0.22$ \\
$7 \mathrm{~T}$ (simulated) & $0.84 \pm 0.07$ & $1.48 \pm 0.11$ & $1.60 \pm 0.11$ & $3.10 \pm 0.24$ \\
\hline
\end{tabular}

Table 6.1: Measured and simulated results for a $0.25 \mathrm{~mm}$ diameter ${ }^{22} \mathrm{Na}$ point source and a $1.0 \mathrm{~mm}$ diameter ${ }^{68} \mathrm{Ga}$ point source in water.

\begin{tabular}{cccccc}
\multicolumn{7}{c}{${ }^{22}$ Na Results } \\
$\begin{array}{c}\text { Slit Width } \\
(\mathrm{mm})\end{array}$ & $\begin{array}{c}\text { B-field } \\
(\text { Tesla })\end{array}$ & $\begin{array}{c}\text { Our Results } \\
\text { FWHM } \\
(\mathrm{mm})\end{array}$ & $\begin{array}{c}\text { Our Results } \\
\text { FWTM } \\
(\mathrm{mm})\end{array}$ & $\begin{array}{c}\text { Hammer } \\
\text { FWHM } \\
(\mathrm{mm})\end{array}$ & $\begin{array}{c}\text { Hammer } \\
\text { FWTM } \\
(\mathrm{mm})\end{array}$ \\
\hline 0.2 & - & - & - & - & - \\
0.7 & 0.0 & $0.90 \pm 0.08$ & $1.6 \pm 0.14$ & - & - \\
1.0 & 0.0 & - & - & $0.96 \pm 0.02$ & $2.25 \pm 0.02$ \\
\hline
\end{tabular}

Table 6.2: Experimental results of the reconstructed positron range for both our small animal PET prototype and for Hammer and Christensen's measurement for ${ }^{22} \mathrm{Na}$ [55]. The diameter of the Hammer and Christensen source is $0.5 \mathrm{~mm}$ while a $0.25 \mathrm{~mm}$ diameter point source was used in this experiment.

\subsection{Artifacts from off-plane source measurements}

The data collected thus far has consisted of point sources confined to be within the detection plane. In actual imaging applications the source distribution will be spread throughout the object. It will not be confined to a single plane. The question must be addressed: how are in-plane PET images affected when positrons from sources located in neighboring planes annihilate within the detection plane? 


\begin{tabular}{llllll}
\multicolumn{5}{c}{} & ${ }^{68}$ Ga Results \\
$\begin{array}{l}\text { Slit Width } \\
(\mathrm{mm})\end{array}$ & $\begin{array}{l}\text { B-field } \\
\text { (Tesla) }\end{array}$ & $\begin{array}{l}\text { Our Results } \\
\text { FWH }\end{array}$ & $\begin{array}{l}\text { Our Results } \\
\text { FWTM } \\
(\mathrm{mm})\end{array}$ & $\begin{array}{l}\text { Hammer } \\
\text { FWHM } \\
(\mathrm{mm})\end{array}$ & $\begin{array}{l}\text { Hammer } \\
\text { FWTM } \\
(\mathrm{mm})\end{array}$ \\
\hline 0.2 & 0.0 & - & - & $2.00 \pm 0.10$ & $6.50 \pm 0.33$ \\
0.7 & 0.0 & $2.3 \pm 0.12$ & $5.7 \pm 0.29$ & - & - \\
1.0 & 0.0 & - & - & $2.30 \pm 0.10$ & $7.10 \pm 0.10$ \\
0.2 & 5.0 & - & - & $1.40 \pm 0.07$ & $3.00 \pm 0.15$ \\
1.0 & 5.0 & - & - & $1.62 \pm 0.05$ & $3.40 \pm 0.05$ \\
0.7 & 7.0 & $1.60 \pm 0.11$ & $3.10 \pm 0.22$ & - & - \\
0.2 & 9.3 & - & - & $0.93 \pm 0.05$ & $1.88 \pm 0.09$ \\
1.0 & 9.3 & - & - & $1.12 \pm 0.03$ & $2.22 \pm 0.03$ \\
\hline
\end{tabular}

Table 6.3: Experimental results of the reconstructed positron range for both our small animal PET prototype and for Hammer and Christensen's measurement for ${ }^{68} \mathrm{Ga}[56]$

In order to determine the effects off-plane sources have on in-plane PET images, data was collected at both $0 \mathrm{~T}$ and $7 \mathrm{~T}$ for four $1.0 \mathrm{~mm}$ diameter ${ }^{68} \mathrm{Ga}$ point sources. The point sources were positioned within the $1.0 \mathrm{~mm}$ thick silicon detector plane and at $1 \mathrm{~mm}, 2 \mathrm{~mm}$, and $3 \mathrm{~mm}$ above the detection plane. Each source is shifted by 45 degrees with respect to its nearest neighbors. The reconstructed ML-EM images for the measured data are shown in Fig. 6.14. Figure 6.15 is the projection of these images along the x-axis. Also plotted in Fig. 6.15 are the results of an EGS4 simulation of the identical source configuration in water. The measured and simulated data are in good agreement. The deviation between the measured and simulated data is due to the uncertainty of positioning the positron sources in the direction transverse to the image plane for the measured data.

Note that the artifacts from the off-plane sources are more defined at $7 \mathrm{~T}$ than at 0 T. This is because the positron range is more tightly confined in the transverse plane 

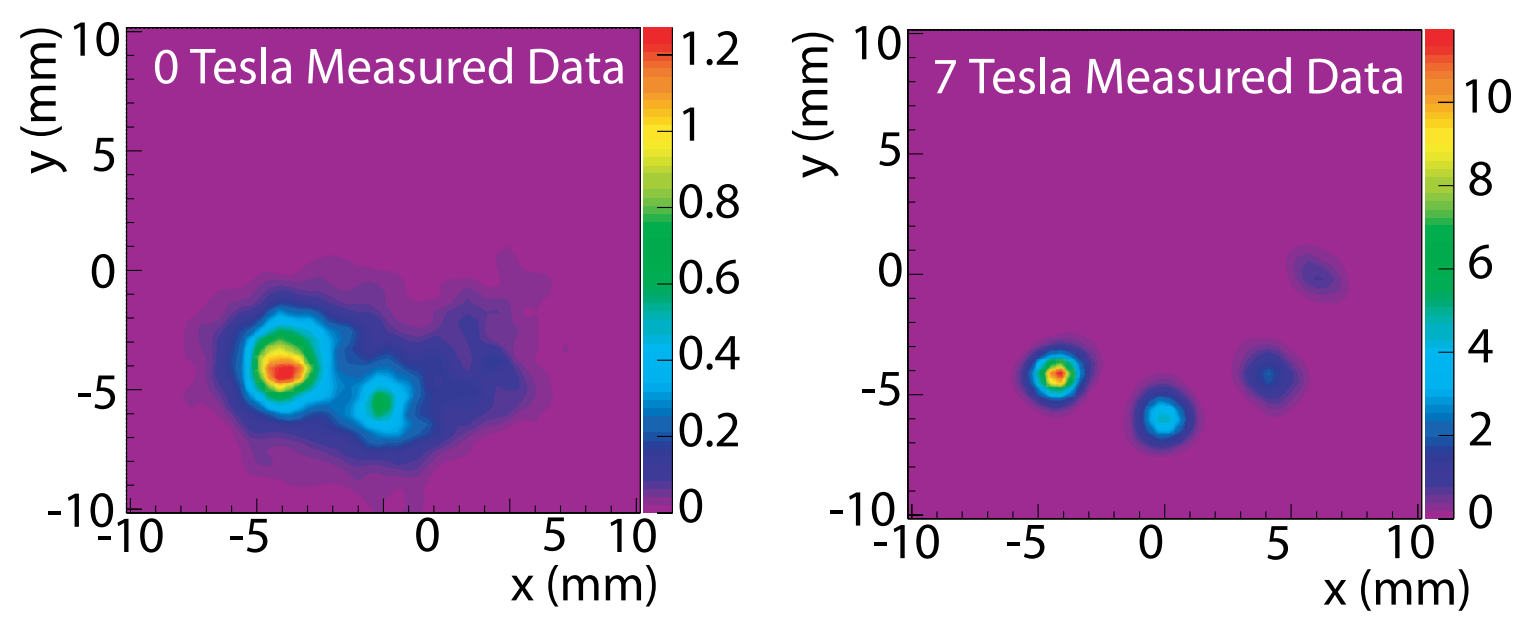

Figure 6.14: Left: Measured data of four $1.0 \mathrm{~mm}$ diameter ${ }^{68} \mathrm{Ga}$ point sources positioned within the detector plane and at $1 \mathrm{~mm}, 2 \mathrm{~mm}$, and $3 \mathrm{~mm}$ outside of the detection plane at $0 \mathrm{~T}$. Right: Measured data of four $1.0 \mathrm{~mm}$ diameter ${ }^{68} \mathrm{Ga}$ point sources positioned within the detector plane and at $1 \mathrm{~mm}, 2 \mathrm{~mm}$, and $3 \mathrm{~mm}$ outside of the detection plane at $7 \mathrm{~T}$ (applied transverse to the image plane). The artifacts from off-plane sources are more sharply defined in the strong magnetic field.

when a strong magnetic field is applied. In other words, what was previously a diffuse background from off-plane sources at $0 \mathrm{~T}$ is a sharp peak at $7 \mathrm{~T}$. The $7 \mathrm{~T}$ images have improved resolution but at the price of more highly defined artifacts from sources positioned off-plane. These results lead to some important questions. Can artifacts caused by off-plane sources be removed by correcting for the range of the positron in the ML-EM reconstruction? If so, then why not use the 3D ML-EM positron range correction algorithm on the $0 \mathrm{~T}$ data to correct for the positron range? If the positron range can be completely removed, is there any benefit to collecting PET data in strong magnetic fields. The following chapter investigates these questions.

It is important to note that the artifacts due to off-plane sources are likely to be more defined using a stack of 2D PET planes to create 3D images in contrast to 

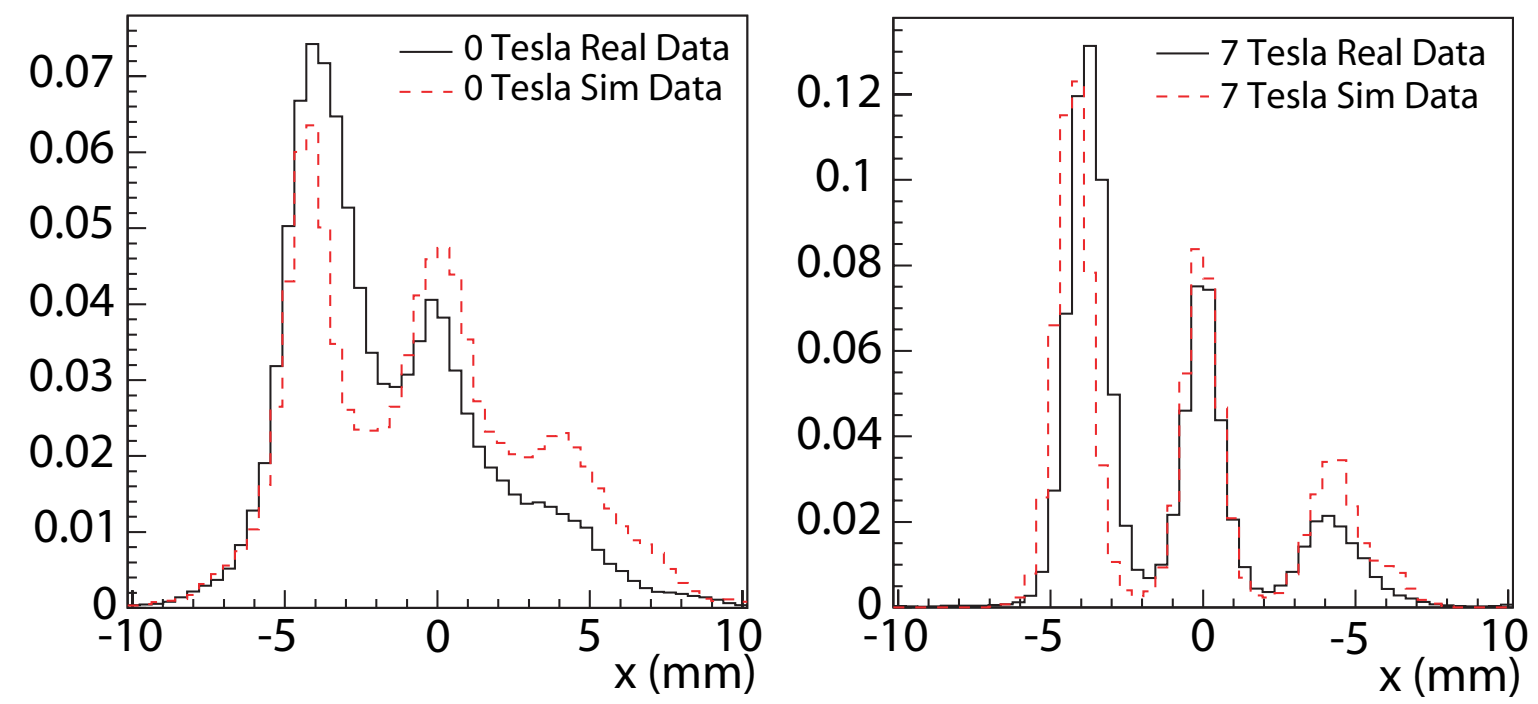

Figure 6.15: Left: Projection along the x-axis of the measured data of four $1.0 \mathrm{~mm}$ diameter Ga-68 point sources positioned within the detector plane and at $1 \mathrm{~mm}, 2$ $\mathrm{mm}$, and $3 \mathrm{~mm}$ outside of the detection plane at $0 \mathrm{~T}$. Right: Projection along the $\mathrm{x}$-axis of the measured data of four $1.0 \mathrm{~mm}$ diameter Ga-68 point sources positioned within the detector plane and at $1 \mathrm{~mm}, 2 \mathrm{~mm}$, and $3 \mathrm{~mm}$ outside of the detection plane at $7 \mathrm{~T}$ (applied transverse to the image plane). The artifacts from off-plane sources are more sharply defined in the strong magnetic field. 


\section{a). Pseudo 3D PET}

(Stack of 2D PET slices)

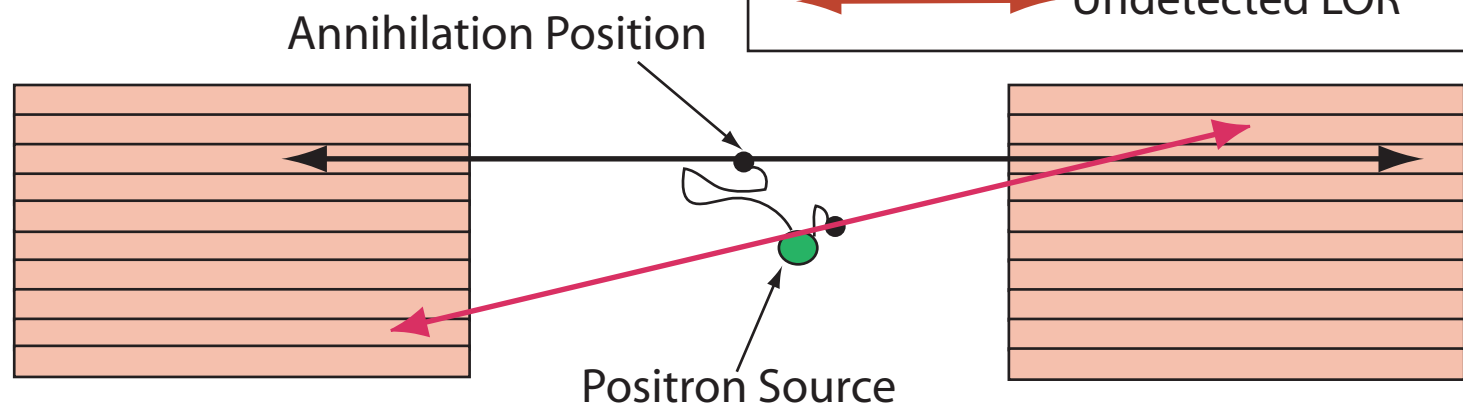

b). Actual 3D PET

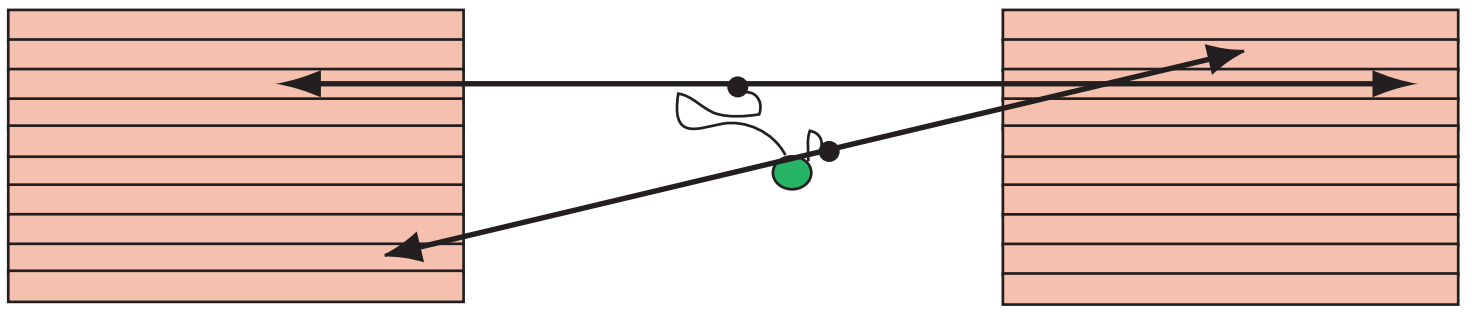

Figure 6.16: A diagram demonstrating the LORs that can be detected using a). a stack of 2D PET detectors to create 3D images and b). the LORs that would be detected in a fully 3D PET system. 
a fully 3D PET system. The reason for this is shown in Fig. 6.16. In this figure a positron source is positioned within the detector volume. Two annihilation events are shown that demonstrate the relevant effects that will lead to more sharply defined artifacts for the $2 \mathrm{D}$ case. Figure a) is a diagram that is analogous to the previous measurements. For this case only positron annihilations that result in photon pairs emitted within one of the $2 \mathrm{D}$ detector planes will be detected. This is demonstrated by the black arrow (a detected event) and the red arrow (an undetected event). In contrast, a fully 3D PET system can detect photon pairs emitted in a PET plane and photon pairs that span planes (this is shown in case b). It has been demonstrated that the number of positron-electron annihilations per unit volume increases for positions closer to the positron source (see the positron-electron annihilation density plots shown in Figs. 2.11 through 2.17). The ratio of the number of annihilation events detected from the tails of the positron-electron annihilation distribution to the number of annihilation events occurring close to the positron source will be greater for 3D PET using a stack of 2D planes than for a fully 3D PET system. Thus, the tails of the annihilation distribution due to off-plane sources will be given a strong weight for pseudo 3D PET systems (a stack of 2D planar PET images), leading to more defined artifacts due to off-plane sources. The simulation results discussed in chapter 7 are specific to 3D PET systems that only record coincidence events from photon pairs emitted in each 2D plane. 


\section{CHAPTER 7}

\section{Resolution Noise Study}

\subsection{Purpose of Chapter}

The purpose of this chapter is to investigate the image quality of $3 \mathrm{D}$ simulated data reconstructed with the 3D ML-EM positron range correction algorithm for high kinetic energy positron emitters $\left({ }^{68} \mathrm{Ga}\right)$. Three separate cases are tested for each study presented in this chapter in order to determine if there is an advantage to collecting data with a $7 \mathrm{~T}$ magnetic field direction in the axial direction (transverse to the image plane) or with data collected independently in three orthogonal multiple magnetic field orientations in comparison to $0 \mathrm{~T}$ positron range corrected images. All three cases use the same 3D ML-EM positron range correction algorithm (discussed in section 5.3); however, different positron range distributions are used as inputs to the algorithm depending on the magnitude and direction of the applied magnetic field used to create the simulated data. The following list details the there cases shown in Fig 7.1:

- Case 1 (0 T): The $0 \mathrm{~T}^{68} \mathrm{Ga}$ positron range distribution in water is used to simulate the data and as input to the 3D ML-EM positron range correction algorithm. 
- Case 2 (7 T, axial) : The ${ }^{68} \mathrm{Ga}$ positron range distribution in water in the presence of a $7 \mathrm{~T}$ magnetic field directed transverse to the image planes (axially) is used to simulate the data and as input to the 3D ML-EM positron range correction algorithm. This case is analogous to PET/MRI systems [57].

- Case 3 (7 T, three orientations: Three independent data sets are collected with the ${ }^{68} \mathrm{Ga}$ positron range distribution in water in the presence of a $7 \mathrm{~T}$ magnetic field directed along the $\mathrm{x}, \mathrm{y}$, and $\mathrm{z}$ axis. Each data set is simulated independently, eventually yielding three sinogram stacks (instead of one stack, as in the previous two cases). The three sinogram stacks (with the B-field directed along either the $\mathrm{x}, \mathrm{y}$, or $\mathrm{z}$ axis) are reconstructed simultaneously using the corresponding $\mathrm{x}, \mathrm{y}$, or $\mathrm{z}^{68} \mathrm{Ga}$ positron range distribution in the 3D ML-EM positron range correction algorithm.

These three cases are tested for a variety of increasingly difficult PET imaging tasks. The first task is to revisit the ${ }^{68} \mathrm{Ga}$ off-plane measurements (see section 6.5 ) and demonstrate that the positron range contribution to the image blur can be completely corrected for both $0 \mathrm{~T}$ and $7 \mathrm{~T}$ simulated data when using the 3D ML-EM positron range correction algorithm to reconstruct the data (see section 5.3). The image quality of a more complex phantom source is then addressed where it is shown that there is a slight benefit for operating in strong magnetic fields. Finally, the improvement of the image quality in strong magnetic fields is quantified by performing a detailed study of the noise-resolution trade-off between PET systems employing various magnetic field orientations and comparing the results to PET data simulated in the absence of a magnetic field. 


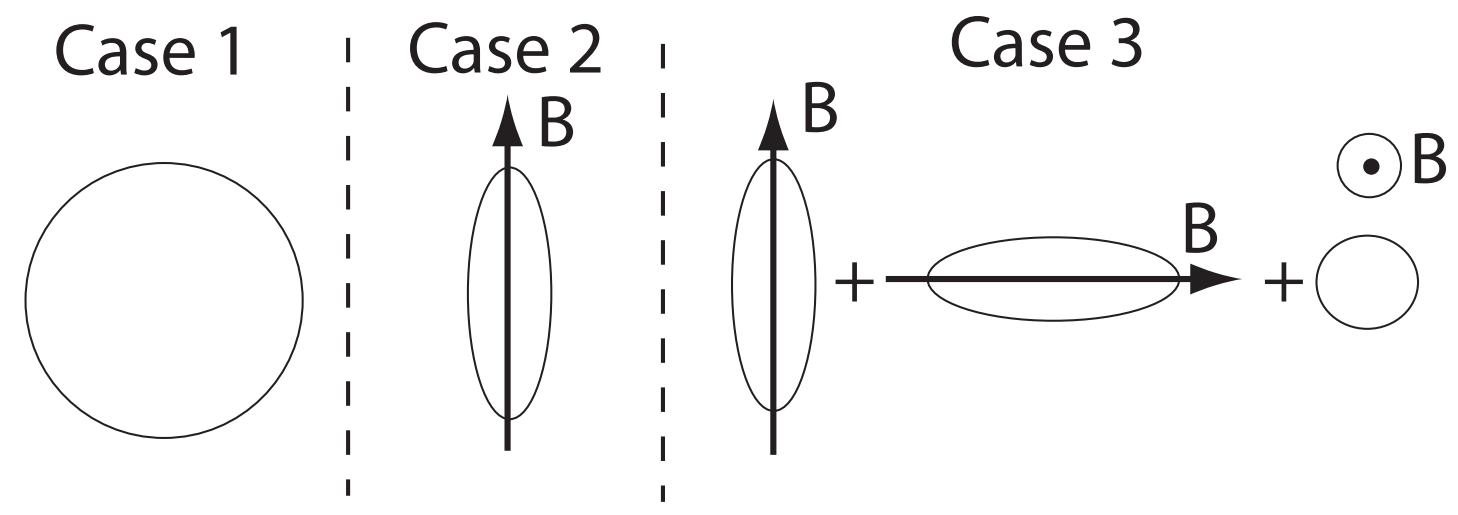

Figure 7.1: This figure illustrates the three cases studied in this chapter. The general shape of the positron range distributions are illustrated in each case. Each case uses the ${ }^{68} \mathrm{Ga}$ positron range distribution with or without the application of a $7 \mathrm{~T}$ magnetic field. Case 1: The $0 \mathrm{~T}^{68} \mathrm{Ga}$ positron range distribution in water is used to simulate the data and as input to the reconstruction algorithm. Case 2: The ${ }^{68} \mathrm{Ga}$ positron range distribution in water in the presence of a $7 \mathrm{~T}$ magnetic field directed transverse to the image planes (axially) is used to simulate the data and as input to the reconstruction algorithm. Case3: Three independent data sets are simulated with the ${ }^{68} \mathrm{Ga}$ positron range distribution in water at $7 \mathrm{~T}$ along the $\mathrm{x}, \mathrm{y}$, and $\mathrm{z}$ axis. The three data sets are reconstructed simultaneously using the $\mathrm{x}, \mathrm{y}$, and $\mathrm{z}$ positron range distributions of ${ }^{68} \mathrm{Ga}$ in water. 


\subsection{Simulating Poisson Distributed Data}

The number of detected events emitted from a particular voxel will vary due to the random nature of positron scattering, annihilation photon emission angle and acollinearity, and the probability of being detected in coincidence. As a result of these random processes the number of events detected from a particular voxel in image space is Poisson distributed. The noise investigated in this chapter is only due to the variations in the number of detected events.

The EGS4 simulation discussed in chapter 2 was designed for single plane detection and has to be modified to account for a stack of silicon detectors. However, equivalent data can be simulated, with far less effort, by blurring 3D source distributions with the positron range (from the EGS4 simulations) and forward projecting the resulting positron annihilation distribution with the system matrix $a(i, j)$ to create the simulated sinograms. Unlike the full EGS4 simulation, this technique does not account for noise due to the Poisson distribution of events, and an extra step is needed to take this into account.

To take into account the variation in the number of detected LORs due to the random Poisson nature of the PET data the intensity of each pixel of the "perfect" sinogram is used as the input to a function that randomly determines an updated sinogram pixel intensity. The original sinogram pixel intensity is used as the mean of the Poisson distribution from which a random sinogram intensity is selected. An example of a sinogram for a single voxel point source blurred with the $0 \mathrm{~T}^{68} \mathrm{Ga}$ positron range without (left) and with (right) 100,000 Poisson distributed events is shown in Fig. 7.2. The "perfect" sinogram on the left has also been blurred with the $0.7 \mathrm{~mm}$ FWHM Gaussian system resolution and the $0.41 \mathrm{~mm}$ blurring due to photon 

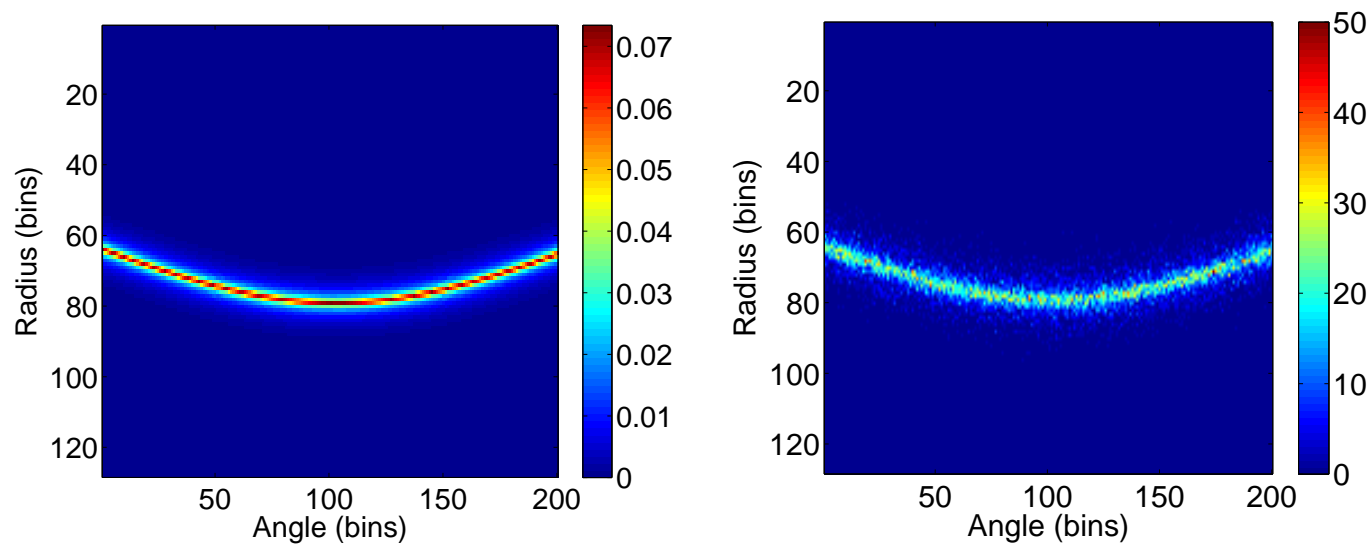

Figure 7.2: Example of sinograms created from a single pixel point source located 6.0 $\mathrm{mm}$ from the center of the FOV blurred with the $0 \mathrm{~T}^{68} \mathrm{Ga}$ positron range without (left) and with (right) Poisson distributed events. The sinogram with Poisson distributed events contains 100,000 LORs. The source image was also blurred with a $0.7 \mathrm{~mm}$ Gaussian system resolution and the $0.41 \mathrm{~mm}$ blur due to photon acollinearity prior to being forward projected into sinogram space in order to compare to the sinograms created from the EGS4 simulation.

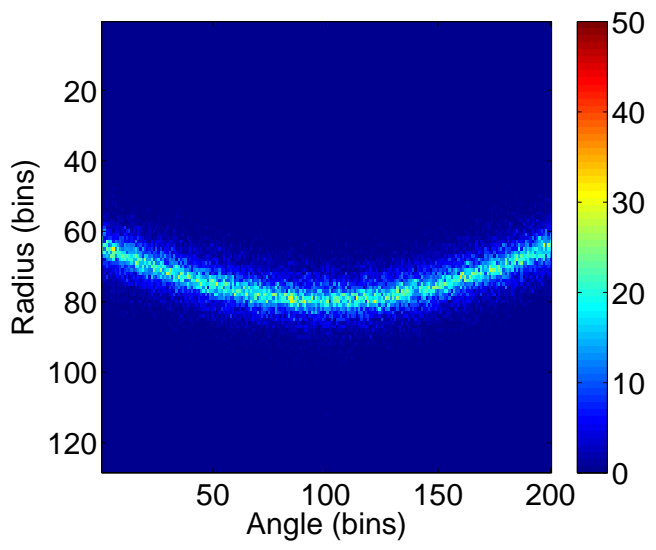

Figure 7.3: Sinogram created from the full EGS4 simulation of a ${ }^{68}$ Ga point source in water at $0 \mathrm{~T}$ located $6.0 \mathrm{~mm}$ from the center of the FOV. This sinogram is used to compare to the sinograms created using the convolution technique discussed in this section. 
acollinearity (prior to applying the Poisson correction) in order to compare the results to a sinogram created using the full EGS4 simulation. Fig. 7.3 is a sinogram created using the full EGS4 simulation for an identical point source at the same location as the source used to create the sinograms in Fig. 7.2. Figure 7.4 is a projection of the sinogram displayed on the right of Fig. 7.2 and the EGS4 sinogram in Fig. 7.3 onto the angle axis. This projection demonstrates the good agreement between the two simulation methods. The difference between the two projections is because EGS4 simulates a full detector ring by rotating the source to discrete positions while the forward projection operation used to create the sinograms via the convolution method assumes a full PET detector ring. The good agreement between these two sinograms demonstrate that the technique discussed in this section to create simulated sinogram data is valid.

\subsection{Testing the 3D ML-EM positron range correction algo- rithm: simple sources}

In this section a simple source distribution similar to the measured and simulated data shown in Figs. 6.14 and 6.15 is revisited using the 3D ML-EM positron range correction algorithm described in chapter 5. The difference between the data simulated in this section and the data presented in section 6.5 is that each image plane is only $0.4 \mathrm{~mm}$ thick (instead of $1.0 \mathrm{~mm}$ thick) and as a result the sources are separated by $0.4 \mathrm{~mm}$ in the axial direction instead of $1.0 \mathrm{~mm}$. The change from $1.0 \mathrm{~mm}$ thick planes to $0.4 \mathrm{~mm}$ planes was chosen in order to more finely model the positron range distribution in the axial direction. As previously observed with both the measured and simulated data, the effect that artifacts from positrons emitted from off-plane sources annihilating within the current detection plane are more prominent for data 


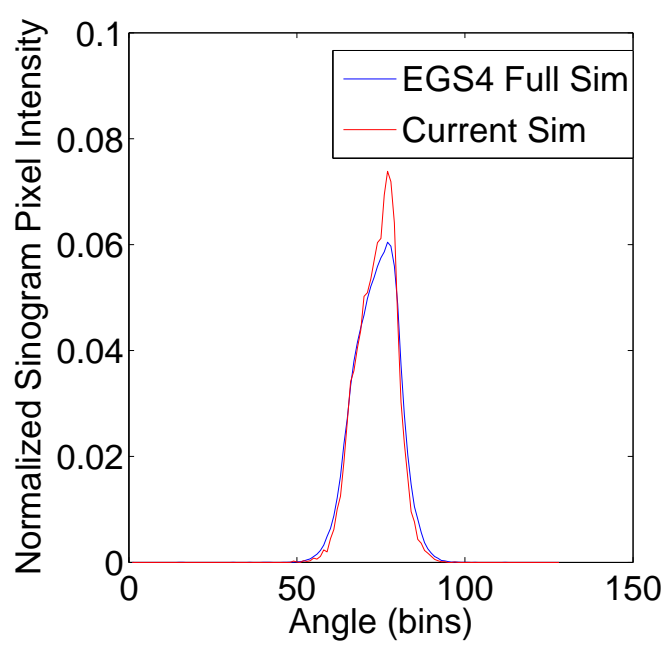

Figure 7.4: Projection of the sinogram shown on the right in Fig. 7.2 and the sinogram from the full EGS4 simulation shown in Fig. 7.3 onto the angular axis. The differences in the sinograms is due to the discrete angular intervals at which the EGS4 data was collected in comparison to the blurring-convolution method that assumes a complete detector ring.

simulated with a $7 \mathrm{~T}$ magnetic field directed transverse to the image plane than in the absence of a magnetic field. Figure 7.5 displays the 3D ML-EM reconstructed images of the four ${ }^{68} \mathrm{Ga}$ point sources with the positron range correction turned off at $0 \mathrm{~T}$ (left) and with a $7 \mathrm{~T}$ magnetic field directed transverse to the image plane (right). Figure 7.6 is the 3D reconstructed images of the same ${ }^{68}$ Ga point source distribution but with the positron range correction turned on. All of the images in this section are post blurred with the $0.7 \mathrm{~mm}$ FWHM system resolution. For the $7 \mathrm{~T}$ reconstructed image, only one orientation of the magnetic field, in the axial direction, was used for the positron range correction. The image on the right of Fig. 7.6 demonstrates that highly defined artifacts from off-plane positron emitters can be statistically corrected by reconstructing the data with a 3D ML-EM positron range correction algorithm. 

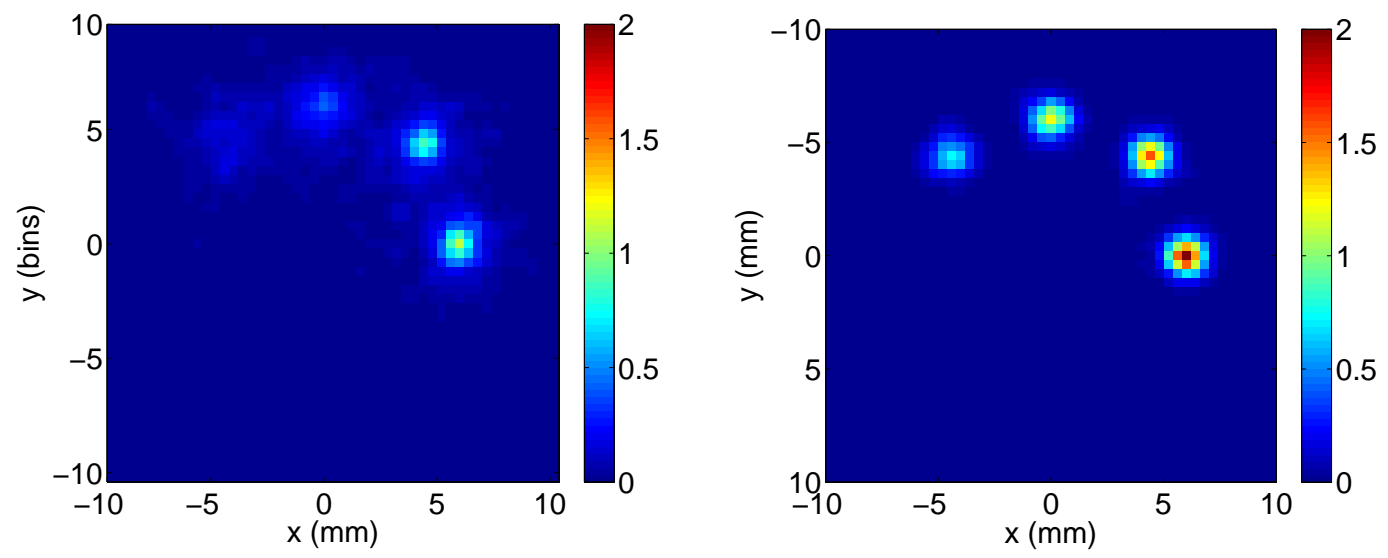

Figure 7.5: Left: 3D ML-EM reconstruction with the positron range correction turned off for simulated data at $0 \mathrm{~T}$ for four $1.0 \mathrm{~mm}{ }^{68} \mathrm{Ga}$ points positioned within the detection plane and at $0.4 \mathrm{~mm}, 0.8 \mathrm{~mm}$, and $1.2 \mathrm{~mm}$ outside of the detection plane. Right: 3D ML-EM reconstruction with the positron range correction turned off for simulated data at $7 \mathrm{~T}$ for four $1.0 \mathrm{~mm}{ }^{68} \mathrm{Ga}$ points positioned within the detection plane and at $0.4 \mathrm{~mm}, 0.8 \mathrm{~mm}$, and $1.2 \mathrm{~mm}$ outside of the detection plane. Each image contains 100,000 Poisson distributed events. These images show that the artifacts from off-plane sources are more sharply defined in a strong magnetic field directed transverse to the image plane. 

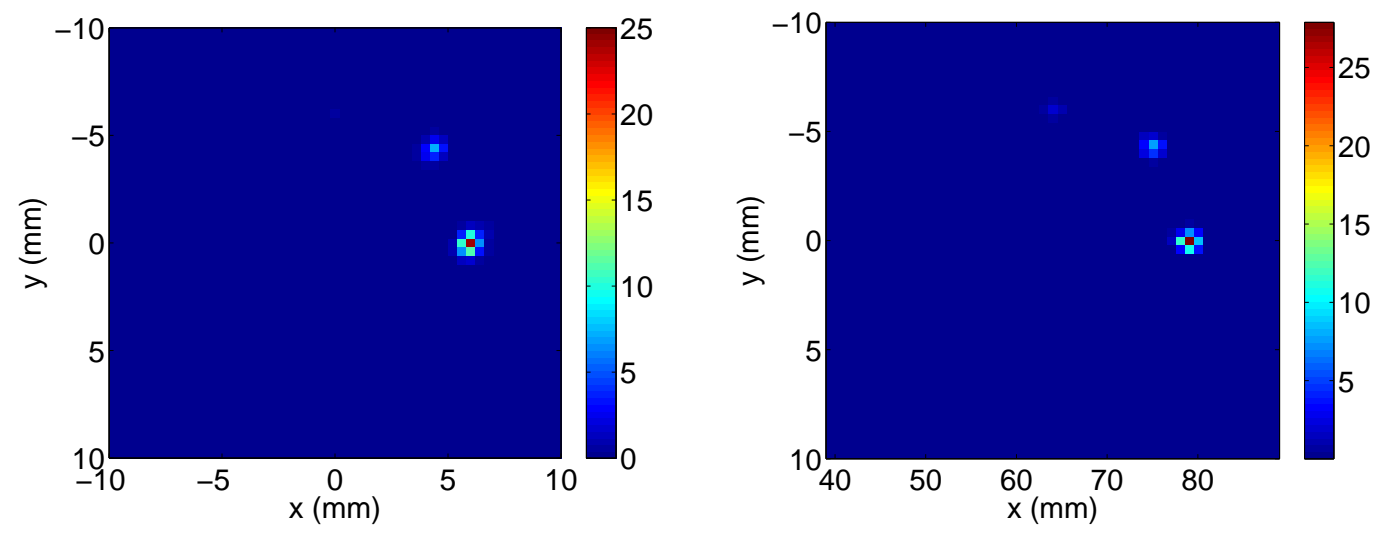

Figure 7.6: Left: 3D ML-EM reconstruction with the positron range correction turned on for data simulated at $0 \mathrm{~T}$ for four $1.0 \mathrm{~mm}{ }^{68} \mathrm{Ga}$ point sources positioned within the detection plane and at $0.4 \mathrm{~mm}, 0.8 \mathrm{~mm}$, and $1.2 \mathrm{~mm}$ outside of the detection plane. Right: 3D ML-EM reconstruction with the positron range correction turned on (using the $7 \mathrm{~T}$ (axial) correction) for simulated data at $7 \mathrm{~T}$ for four $1.0 \mathrm{~mm}$ ${ }^{68} \mathrm{Ga}$ point sources positioned within the detection plane and at $0.4 \mathrm{~mm}, 0.8 \mathrm{~mm}$, and $1.2 \mathrm{~mm}$ outside of the detection plane. Each image contains 100,000 Poisson distributed events. The positron range contribution to the final image resolution has been completely removed for both the $0 \mathrm{~T}$ and $7 \mathrm{~T}$ data. 

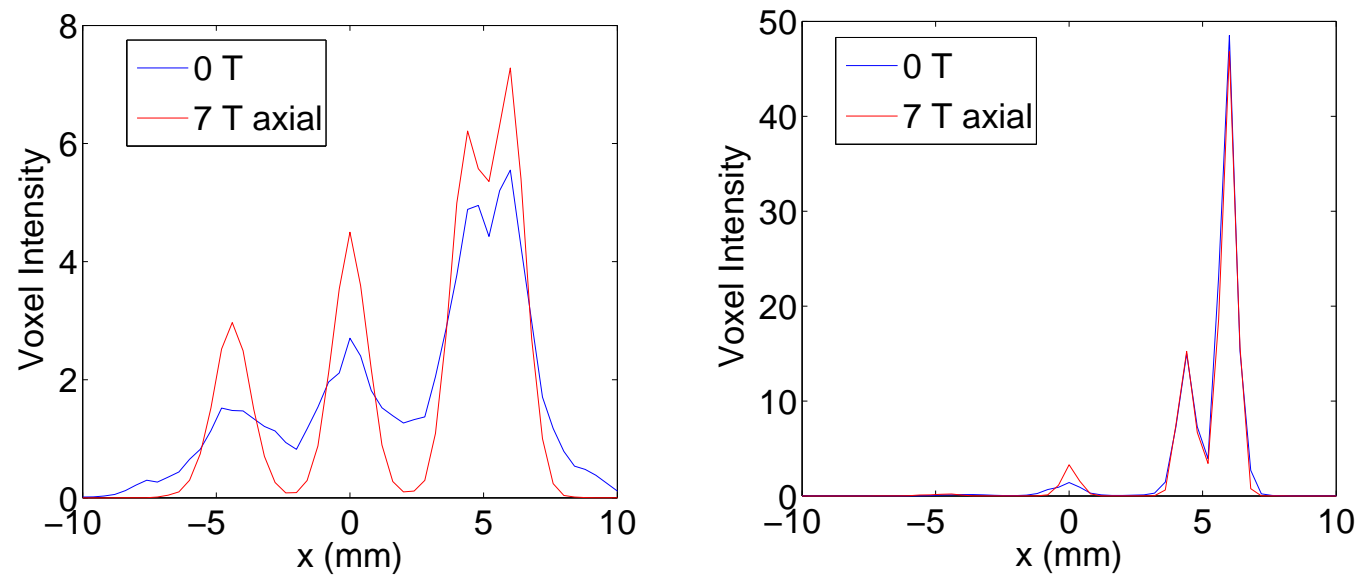

Figure 7.7: Left: The projection of the two images contained in Fig. 7.5 onto the $\mathrm{x}$-axis demonstrating that the artifacts from positron annihilations within the current image plane from sources located in adjacent planes are more highly defined in strong magnetic fields. Right: The projection of the two images contained in Fig. 7.6 onto the $\mathrm{x}$-axis demonstrating that the positron range has been completely removed. The artifacts still present from off-plane sources is due to the $0.7 \mathrm{~mm}$ FWHM Gaussian system resolution and the $0.41 \mathrm{~mm}$ photon acollinearity uncertainty. 

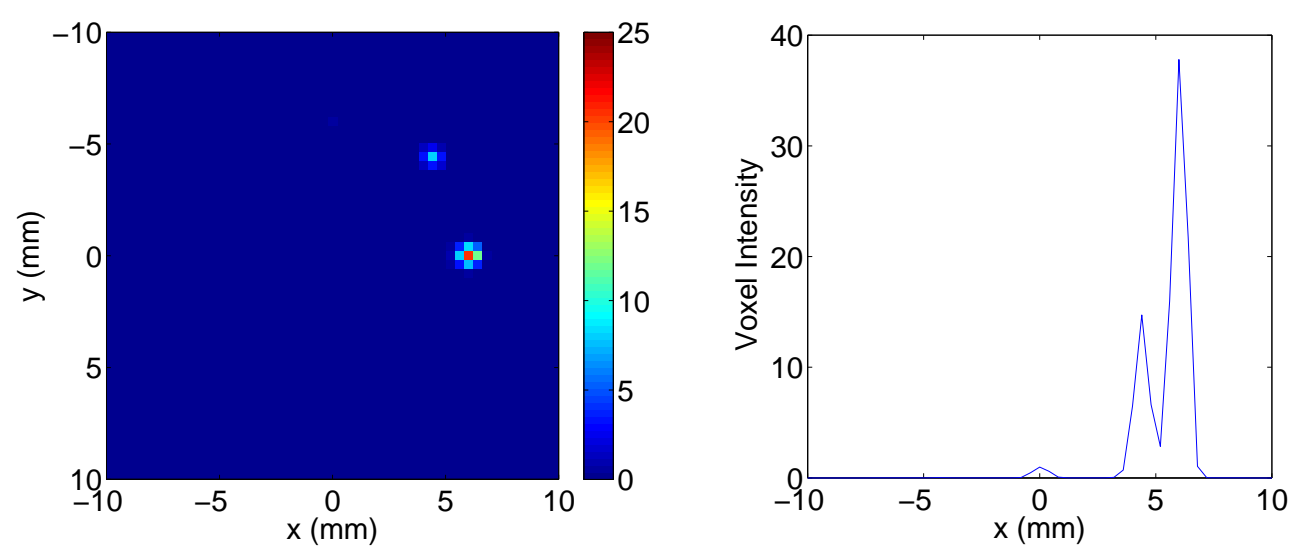

Figure 7.8: Left: 3D ML-EM positron range corrected image using three, independent magnetic field orientations along the $\mathrm{x}, \mathrm{y}$, and $\mathrm{z}$ axes. This image has been blurred with the $0.7 \mathrm{~mm}$ FWHM Gaussian system resolution and the $0.41 \mathrm{~mm}$ uncertainty introduced by the photon acollinearity. Right: The x projection of the image on the left.

The image on the left of Fig. 7.8 is the reconstructed 3D ML-EM positron range corrected image of the source using three independent data sets simultaneously with the magnetic field directed along either the $\mathrm{x}, \mathrm{y}$ or $\mathrm{z}$ axis. The plot on the right of Fig. 7.8 is a projection of the adjacent image onto the $\mathrm{x}$ axis. These images demonstrate that using three orthogonal orientations of the magnetic field yields nearly identical results as the $0 \mathrm{~T}$ and $7 \mathrm{~T}$ axial positron range corrected images.

It has been demonstrate in this section that for simple source distributions there is no apparent advantage to using strong magnetic fields to confine the range of the positron when a 3D ML-EM positron range correction algorithm is used to reconstruct the data. These results also indicate that artifacts caused by off-plane positron emitting sources can be statistically removed in the during the image reconstruction, thus improving the image quality of combined MRI/PET system. 


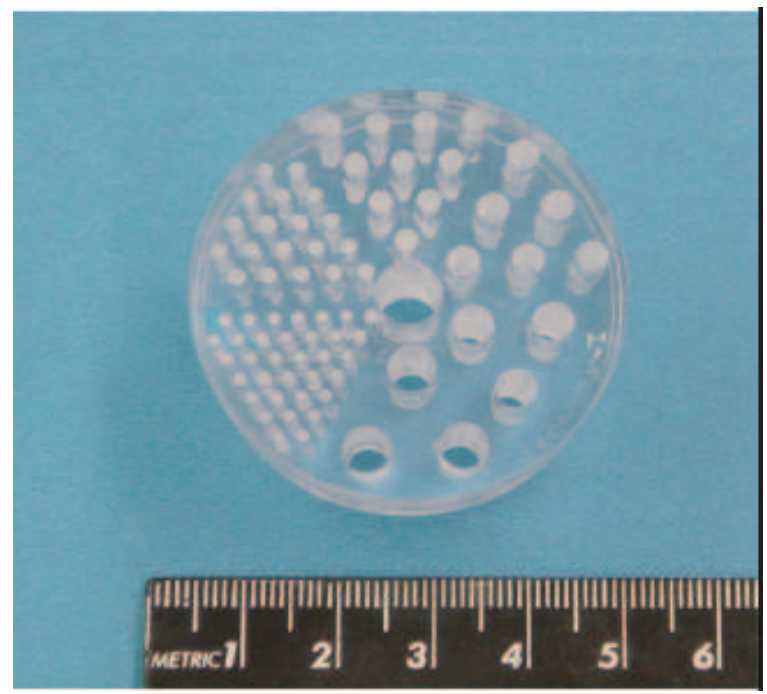

Figure 7.9: A photograph of a complex liquid source holder that is used to characterize the performance of PET scanners (also known as a Jaszczak phantom [58]). The source distribution simulated in this section is based off of this phantom, but with two neighboring planes rotated at an angle with respect to each other. Photograph courtesy of Sang-June Park [44].

\subsection{Testing the 3D ML-EM positron range correction algo- rithm: complex sources}

It was shown in the previous section that the positron range is not difficult to statistically correct for when imaging simple point sources. The question still remains: does the 3D ML-EM positron range correction algorithm perform equally well for more complex sources? This question is addressed by examining the image quality of simulated data from a complex phantom reconstructed with the 3D ML-EM positron range correction algorithm. A phantom is a standardized source distribution that is used to characterize and compare the image quality between various PET systems. There are many types of phantoms that can be used. 

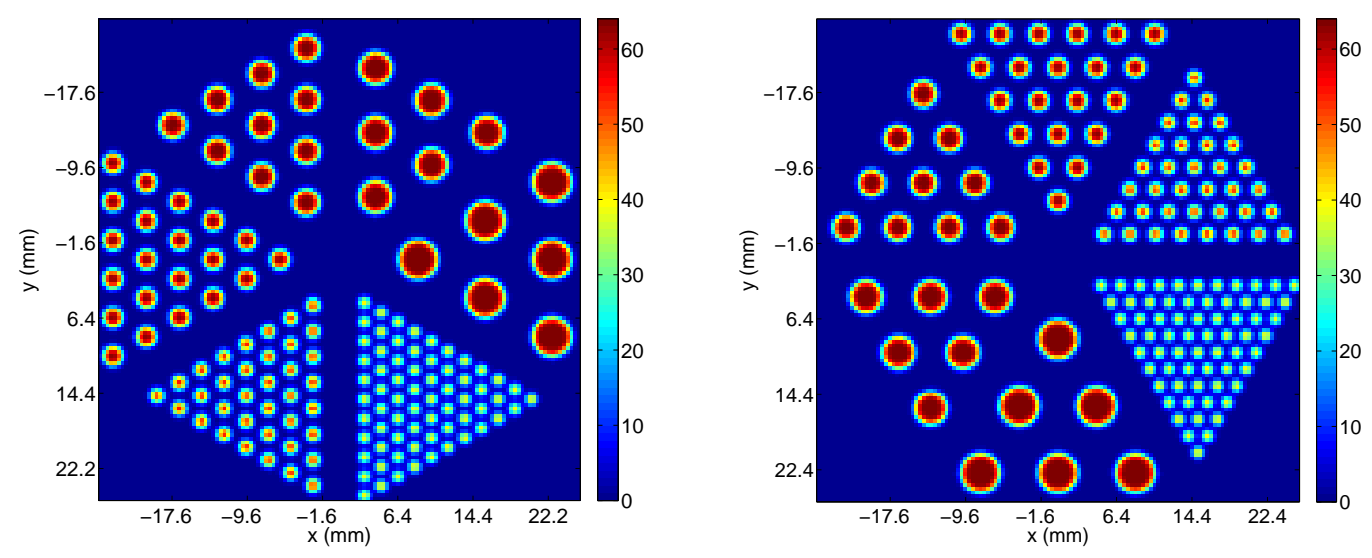

Figure 7.10: Phantom source distribution for plane 5 and 7 of a ten plane phantom. Each plane is $0.4 \mathrm{~mm}$ thick. All other planes do not contain positron sources.

The source distribution simulated in this section is based off of the phantom shown in Fig. 7.9. This phantom is composed of water equivalent plastic with a series of hollow rods that extend throughout the cylinder. The cross-face of the cylinder is divided into six regions each of which contains rods of a specific diameter: either 4.8 $\mathrm{mm}, 4.0 \mathrm{~mm}, 3.2 \mathrm{~mm}, 2.4 \mathrm{~mm}, 1.6 \mathrm{~mm}$, or $1.2 \mathrm{~mm}$. In each region the rods are separated by twice the diameter of the local rods as measured from the center of two adjacent rods. To perform a PET measurement of the phantom each rod is filled with a liquid radioactive source and then inserted into the PET FOV for data collection.

In this study, the source activity of our simulated phantom does not extend throughout the entire length of the cylindrical volume, but is instead confined to two $0.4 \mathrm{~mm}$ thick planes. In each plane, the phantom source geometry is reproduced, but with the source distributions rotated with respect to each other (see Fig. 7.10). This is done to remove the axial symmetry of the phantom such that artifacts that arise from sources in neighboring planes will be apparent. If the two source planes had 


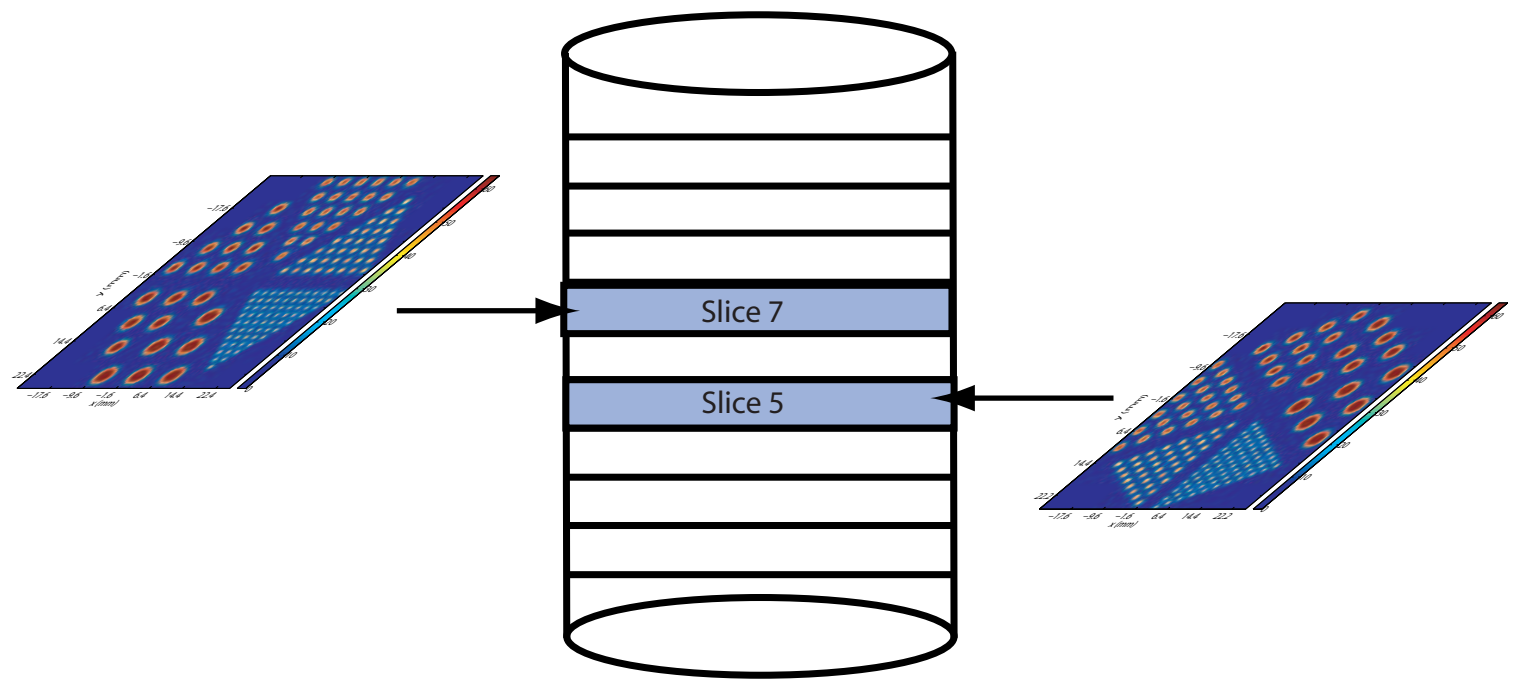

Figure 7.11: A simple illustration of the phantom source consisting of ten $0.4 \mathrm{~mm}$ thick planes. Only planes 5 and 7 contain sources. Note that the sources in planes 5 and 7 are rotated with respect to each other such that positron penetration from neighboring planes will be evident.

not been rotated with respect to each other, then positron-electron annihilations from off-plane sources would line up with the annihilation positions from in-plane sources. Essentially, the artifacts would be hidden by the axial symmetry of the phantom. Planes 5 and 7 were chosen for the source distribution as shown in Fig. 7.11.

The activity of each region containing the various diameter rods is directly proportional to the volume of the rods that contains the liquid source. The relative activities of an individual rod from each region is listed in Table 7.1 (the maximum activity is arbitrarily set to 1.0 ). The challenge of the reconstruction algorithm is to not only resolve the sources themselves, but to also resolve the relative activity of each source.

The phantom shown in Fig. 7.10 contains only the source positions and has not been blurred for the positron range, photon acollinearity, or the detector resolution. 


\begin{tabular}{cc} 
Rod Diameter $(\mathrm{mm})$ & Relative Activity \\
\hline 4.8 & 1 \\
4.0 & 0.69 \\
3.2 & 0.44 \\
2.4 & 0.25 \\
1.6 & 0.11 \\
1.2 & 0.06 \\
\hline
\end{tabular}

Table 7.1: Table of the relative intensity of an individual rod in each region of the phantom shown in Fig. 7.10. The maximum activity is arbitrarily set to one.

This simulation study also ignores image blur due to random and scattered coincidences. In order to demonstrate the effect of the positron range correction algorithm, the phantom sources will initially only be blurred by the positron range. To give a realistic estimate of the imaging performance of the 3D ML-EM positron range correction algorithm, the number of Poisson distributed events simulated for each of the following images was determined using the maximum noise equivalent count rate $^{5}$ performance of the MicroPET R4 small animal PET scanner described in the study by Knoess et. al. [59]. In this study, it is claimed that the maximum noise equivalent count rate for a mouse phantom is $174 \mathrm{kcps}(174,000$ counts per second). This translates into $10^{8}$ true coincidence events when assuming a modest, ten minute scan time.

To meaningfully compare phantom images using various magnetic field strengths and orientations it must be known that the images are nearly converged. For point sources one can plot the resolution of the source peak versus iteration number to see if the ML-EM algorithm has converged. However, for complex sources this technique for

\footnotetext{
${ }^{5}$ The noise equivalent count rate is a measure of the number of true coincidences that are detected per unit time taking into account the random and scattered coincidence rates.
} 
determining the convergence of the image is not as straight forward. What source do you use for the resolution? Does each source converge at the same rate? The answers to these questions will depend on the source itself. A better criteria for determining when an image has converged is to plot the minimum value of the log-likelihood versus iteration number. As discussed in chapter 5, the log-likelihood is a measure of the probability that the current image matches the measured data. A numeric value for the log-likelihood of the current image estimate can be found by computing the sum in equation 5.5 (in this 3D case, it will be the sum of ten log-likelihoods, one for each image plane). The criteria for convergence is when the difference in the log-likelihood functions between two iterations is small

$$
\left|\ln \left(L^{(k)}\right)-\ln \left(L^{(k-1)}\right)\right|<\epsilon
$$

where $L$ is the likelihood function from equation $5.6, k$ is the current iteration number, and $\epsilon$ is a small number. The negative of each log-likelihood at each iteration is taken and the log-likelihood is normalized such that it will converge to one. Figure 7.12 is a plot of the normalized log-likelihood versus iteration number for the three cases tested on the phantom. All of the images in this section are reconstructed with 2000 iterations of the 3D ML-EM positron range correction algorithm.

Before the 3D ML-EM positron range correction algorithm can be tested, the source distributions shown in 7.10 must be blurred by the relevant positron range, then forward projected into sinogram space, and finally apply the algorithm that creates the Poisson distribution of events (as previously stated for this phantom study $10^{8}$ Poisson distributed events are simulated). Figures 7.13 through 7.15 are examples of the phantom planes 5, 6, and 7 blurred for the ${ }^{68} \mathrm{Ga}$ positron-electron annihilation distribution at $0 \mathrm{~T}$, with a $7 \mathrm{~T}$ magnetic field in the axial z-direction, and with a 


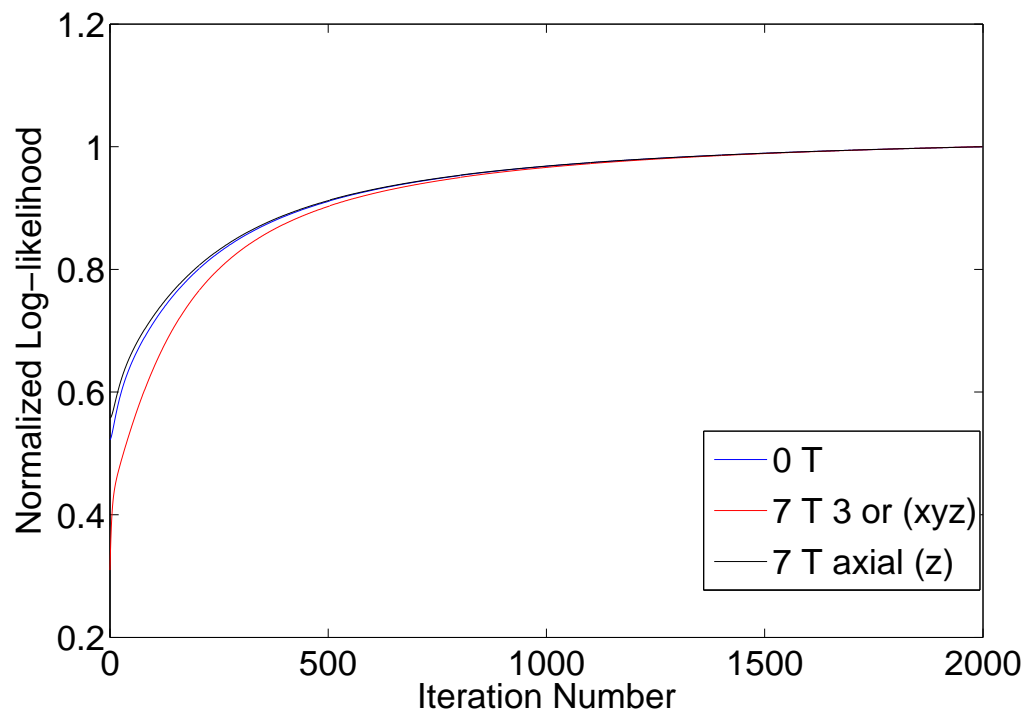

Figure 7.12: Normalized log-likelihood (multiplied by -1 and normalized to converge to 1.0) of the reconstructed phantom versus iteration number for the three cases using the 3D ML-EM positron range correction algorithm.

7 T magnetic field in the y-direction, respectively. Sinograms are created from these distributions by forward projecting into the sinogram domain where the algorithm that creates the simulated Poisson distributed data is applied. The image on the left of Figure 7.16 is an example of a sinogram resulting from the forward projection of plane 5 from the $0 \mathrm{~T}$ positron range blurred phantom. The image on the right of Fig. 7.16 is the same sinogram after the application of the Poisson distribution.

Figures 7.17 through 7.19 are the 3D ML-EM positron range corrected images using the $0 \mathrm{~T}, 7 \mathrm{~T}$ axial (z-direction), and the $7 \mathrm{~T}$ (three orientation xyz) ${ }^{68} \mathrm{Ga}$ positron range distributions. In each figure plane 5 (left), plane 6 (middle), and plane 7 (right) are shown. For each case the positron range contribution to the image blur is nearly removed. Note that for each reconstruction plane 6 does not contain 

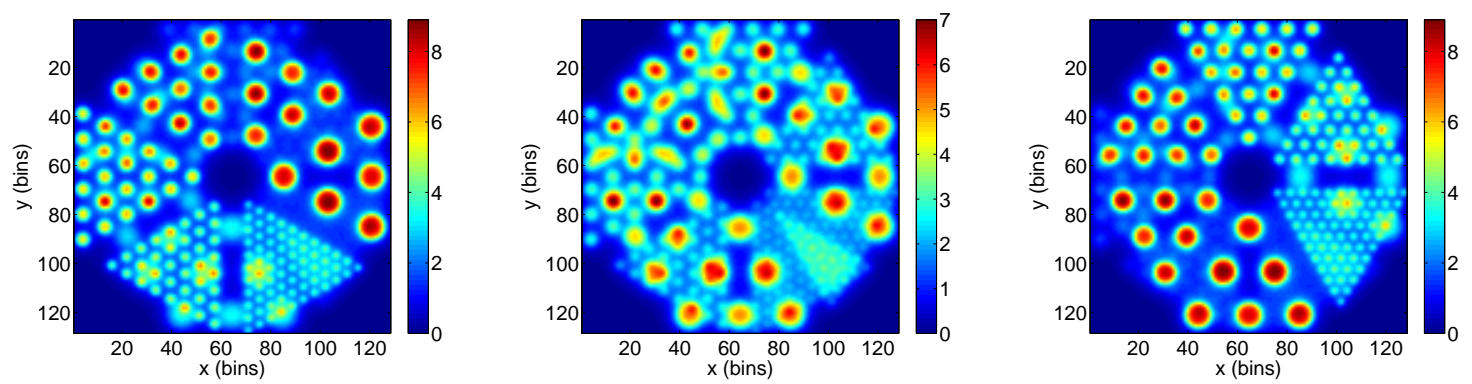

Figure 7.13: Images of phantom planes 5, 6, and 7 (from left to right) blurred with the $0 \mathrm{~T}^{68} \mathrm{Ga}$ positron range distribution in water. Note that plane six does not have any sources and that all annihilation positions are from the two neighboring planes. These are not reconstructed images, but instead are images of the phantoms blurred by the positron range.
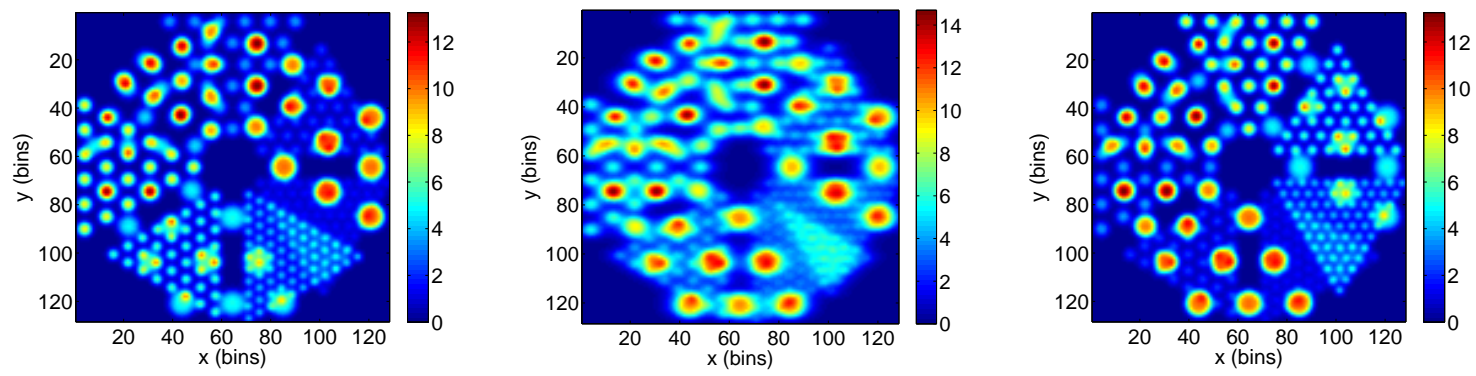

Figure 7.14: Images of phantom planes 5, 6, and 7 (from left to right) blurred with the $7 \mathrm{~T}^{68} \mathrm{Ga}$ positron range distribution in water with a $7 \mathrm{~T}$ magnetic field oriented in the z-direction. Note that plane six does not have any sources and that all annihilation positions are from the two neighboring planes. These are not reconstructed images, but instead are images of the phantoms blurred by the positron range. 

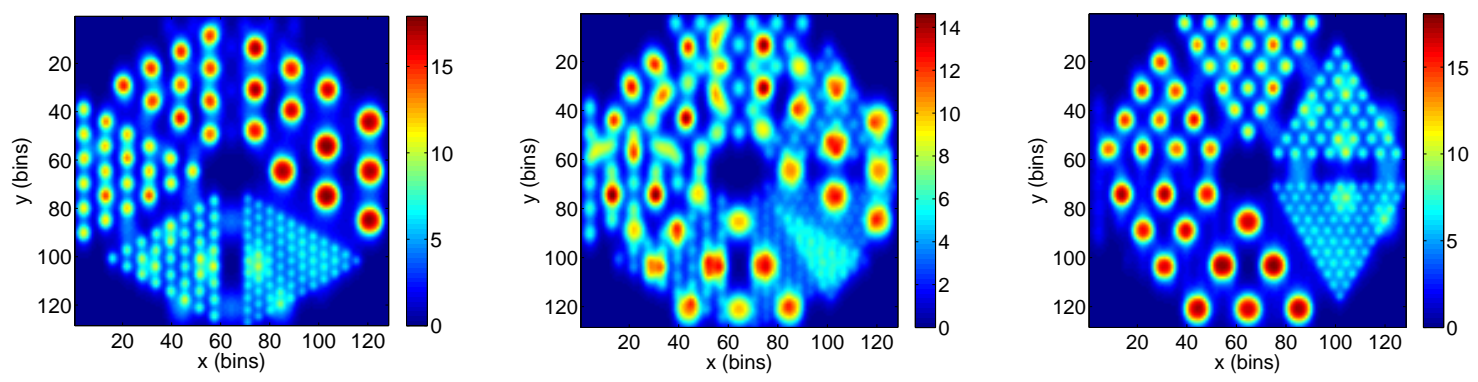

Figure 7.15: Images of phantom planes 5, 6, and 7 blurred with the ${ }^{68}$ Ga positron range distribution in water with a $7 \mathrm{~T}$ magnetic field oriented in the y-direction. Note that plane six does not have any sources and that all annihilation positions are from the two neighboring planes. These are not reconstructed images, but instead are images of the phantoms blurred by the positron range.
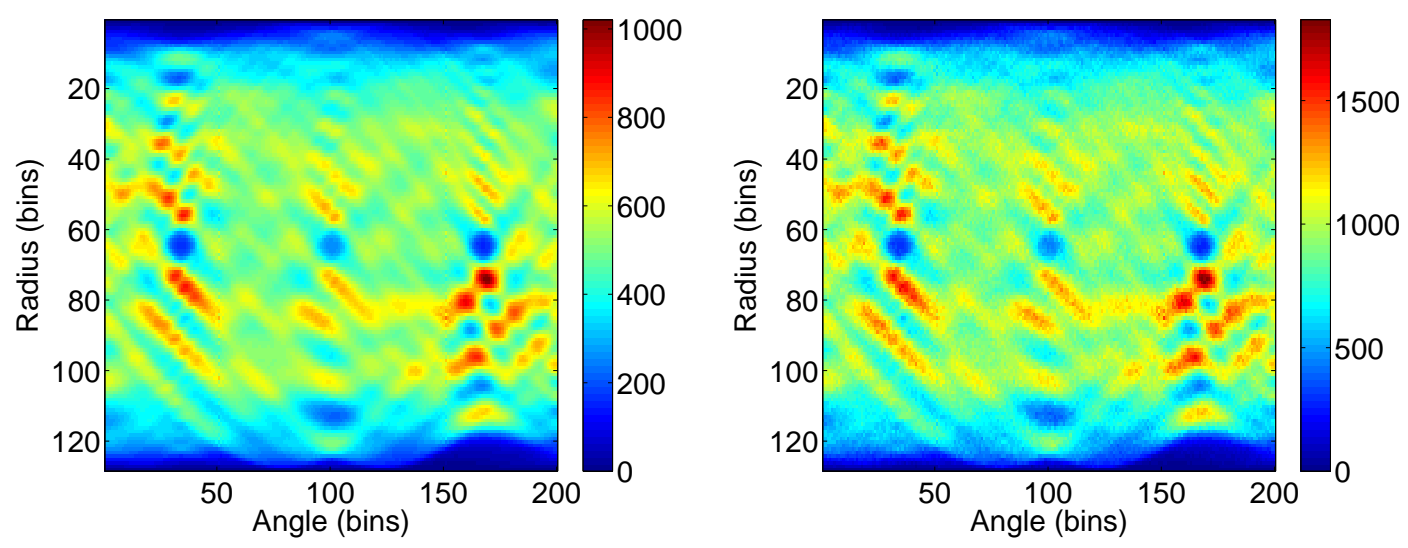

Figure 7.16: Left: Example sinogram created by forward projecting plane 5 of the $0 \mathrm{~T}^{68} \mathrm{Ga}$ blurred phantom. Right: The same sinogram after varying each pixel by a random number from the Poisson distribution. 

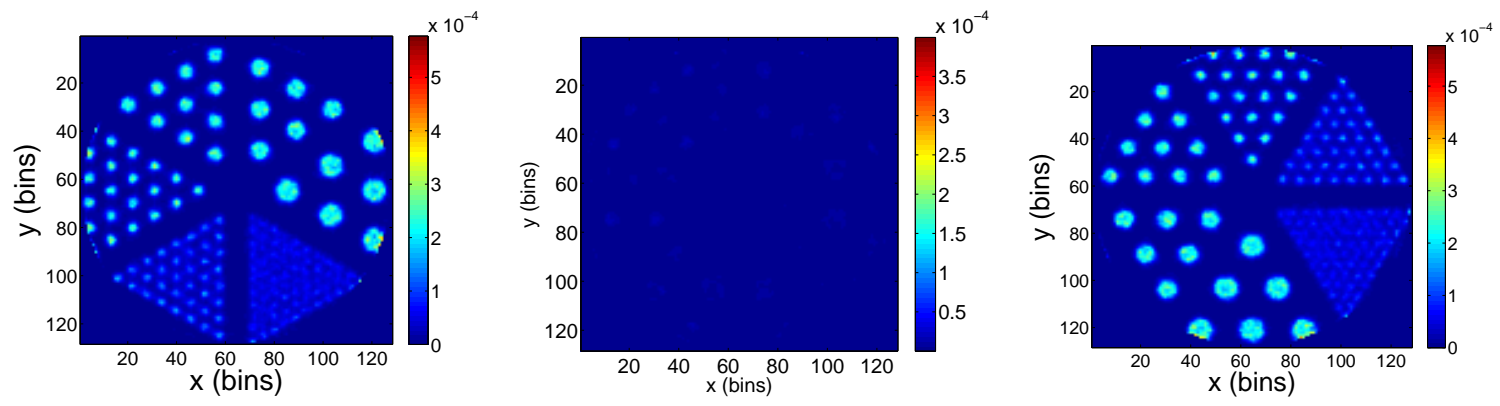

Figure 7.17: Phantom planes 5, 6, and 7 using the $0 \mathrm{~T}^{68} \mathrm{Ga}$ positron range in water for the 3D ML-EM positron range correction algorithm, respectively
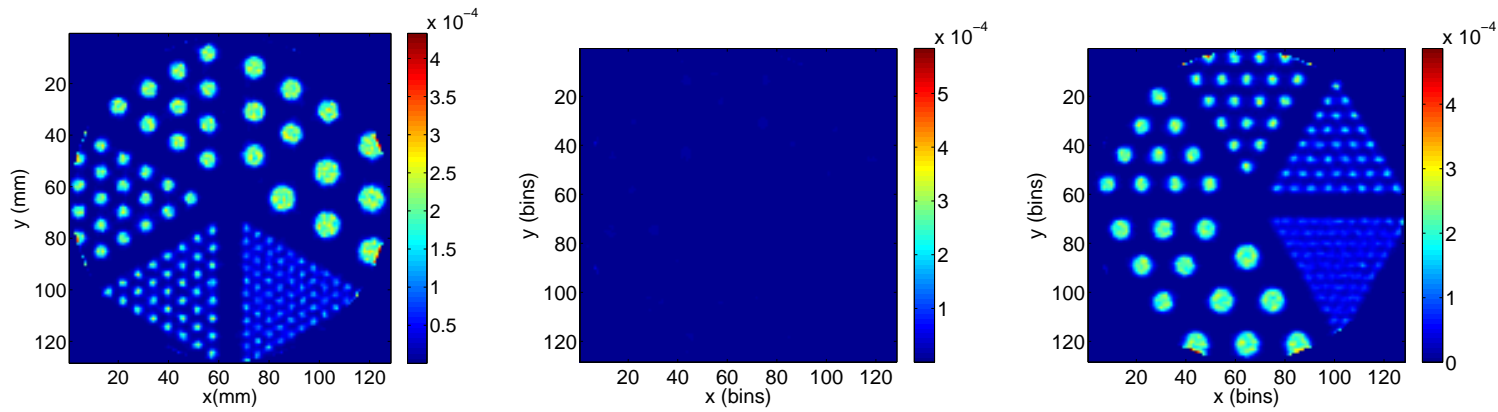

Figure 7.18: Phantom planes 5, 6, and 7 using the $7 \mathrm{~T}^{68} \mathrm{Ga}$ positron range with the magnetic field oriented in the axial z-direction using the 3D ML-EM positron range correction algorithm.

a significant contribution from the neighboring planes, indicating the proper removal of the positron range. From these images it is unclear which, if any, case exhibits improved image quality.

Figures 7.20 displays the zoomed in images of the $1.2 \mathrm{~mm}$ diameter rods from the 3D ML-EM reconstructions shown in Figs. 7.17 through 7.19. Fig. 7.23 are the same images after having been blurred by the 3D $0.7 \mathrm{~mm}$ FWHM Gaussian system resolution. For both of the referenced figures the image on the left is the $0 \mathrm{~T}$ 

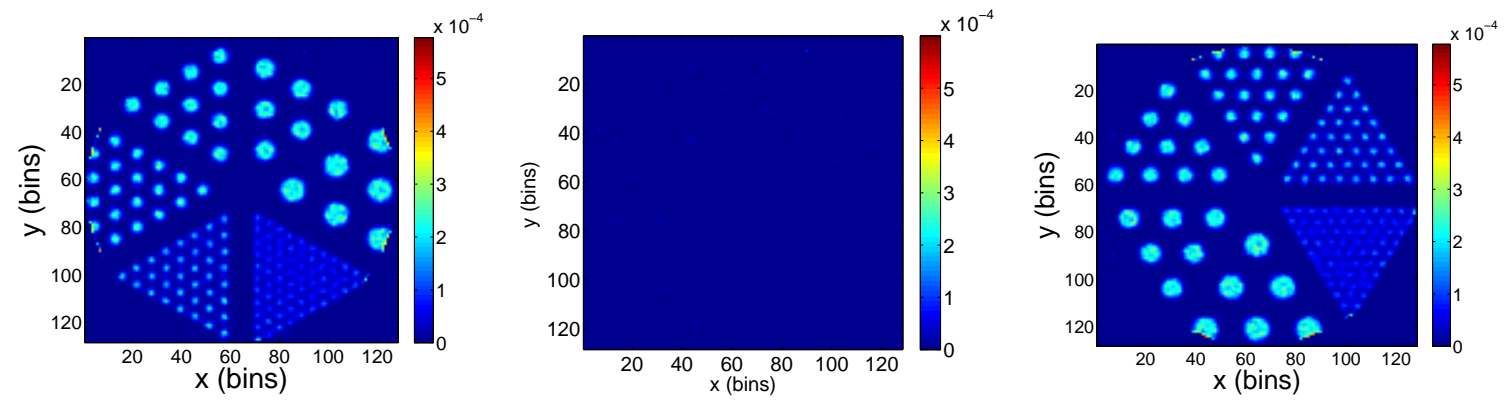

Figure 7.19: Phantom planes 5, 6, and 7 using three orthogonal $7 \mathrm{~T}$ magnetic field orientations of the ${ }^{68} \mathrm{Ga}$ positron range in water for the 3D ML-EM positron range correction algorithm, respectively.

reconstruction, the middle image is from the $7 \mathrm{~T}$ (3 orientation reconstruction), and the image on the right is the $7 \mathrm{~T}$ axial reconstruction. From qualitative observation the $1.2 \mathrm{~mm}$ rods are more easily distinguished using the three orthogonal $7 \mathrm{~T}$ magnetic field orientation reconstruction. This observation is corroborated by investigating a slice that passes through the maxima of a single line of the $1.2 \mathrm{~mm}$ rods as shown in Fig. 7.22. Figures 7.24 through 7.26 are projections along the line shown in Fig. 7.22 where the images on the left are from Fig. 7.20 and the images on the right are from the reconstructions blurred by the system resolution from Fig. 7.23. The black line in these plots represents the true phantom activity, while the blue, red, and green lines represent the $0 \mathrm{~T}, 7 \mathrm{~T}$ (3 orientations), and $7 \mathrm{~T}$ axial reconstructions, respectively. Comparing the projections that have not been blurred by the system resolution (the left plots) it can be seen that the $7 \mathrm{~T}$ (3 orientation) reconstruction has the most defined peaks. However, once the images are blurred for the positron range, all three projections exhibit similar performance. 

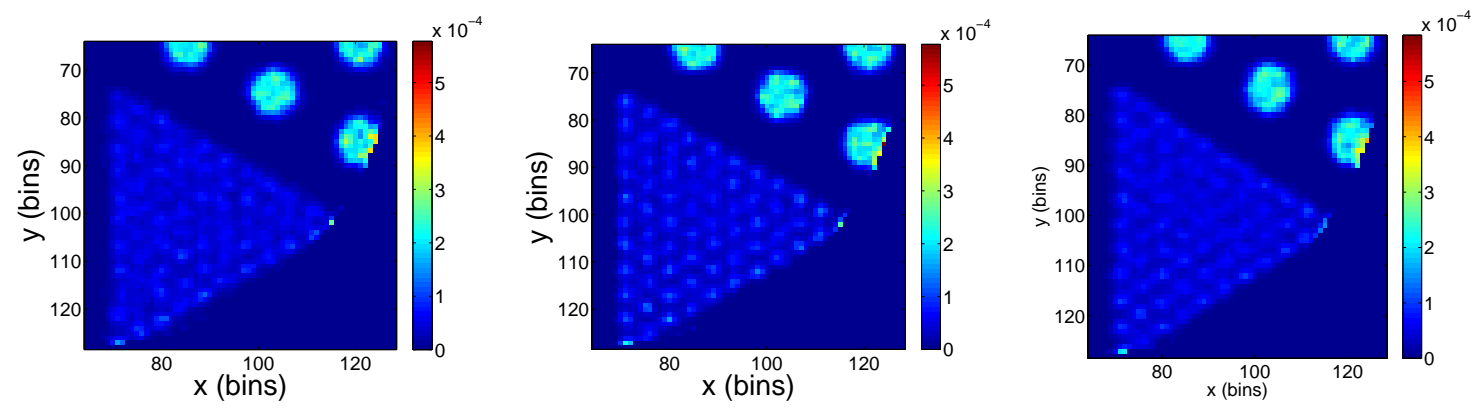

Figure 7.20: $0 \mathrm{~T}$ (left), $7 \mathrm{~T}$ (3 orientations) (middle), and $7 \mathrm{~T}$ (axial) images zoomed in on the small $1.2 \mathrm{~mm}$ diameter sources from the reconstructed images shown in Figs. 7.17 through 7.19 .
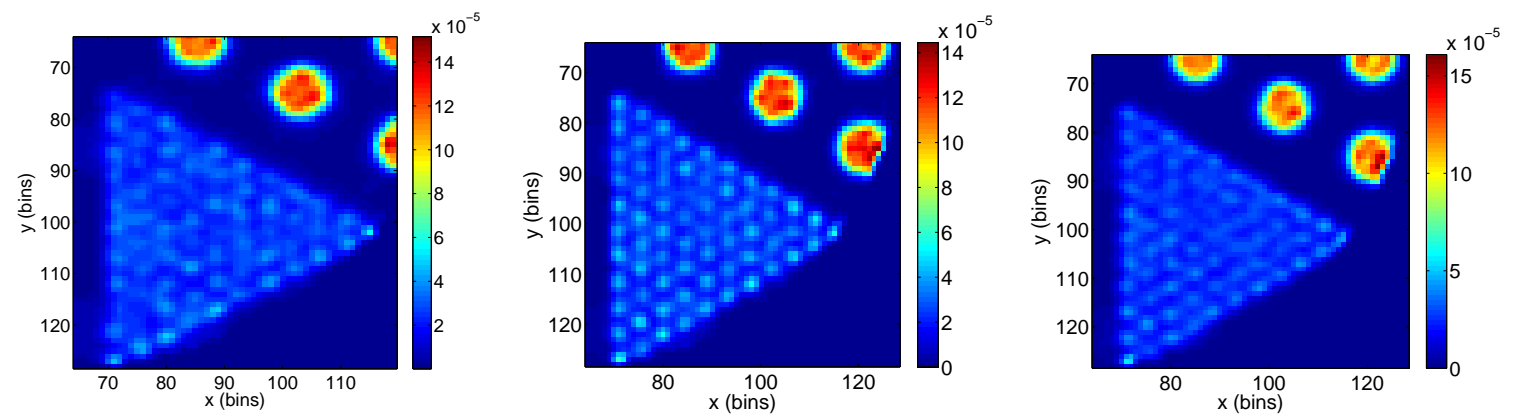

Figure 7.21: Images of $1.2 \mathrm{~mm}$ rods that have been corrected for the positron range with the 3D ML-EM algorithm positron range correction and post blurred for a $0.7 \mathrm{~mm}$ Gaussian system resolution: (left) $0 \mathrm{~T}$, (middle) $7 \mathrm{~T}$ with three orientations, and (left) a $7 \mathrm{~T}$ magnetic field in the axial z-direction. 


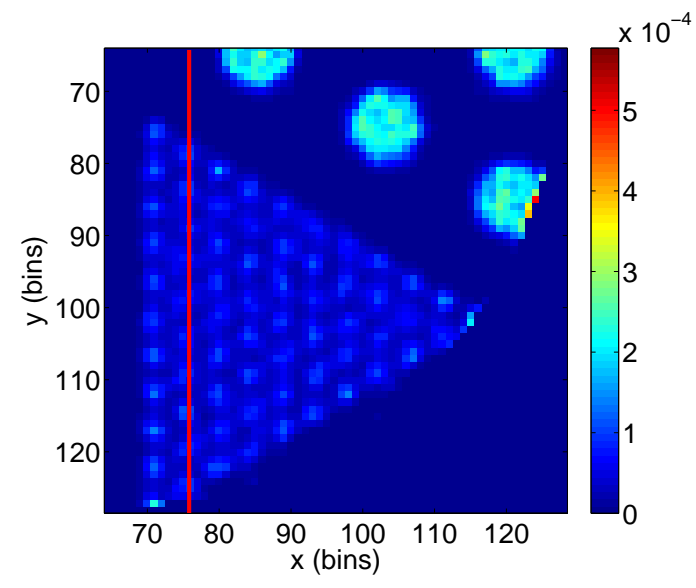

Figure 7.22: The red line represents the projections shown in the following figures for the $1.2 \mathrm{~mm}$ rods shown in Figs. 7.24 through 7.26.
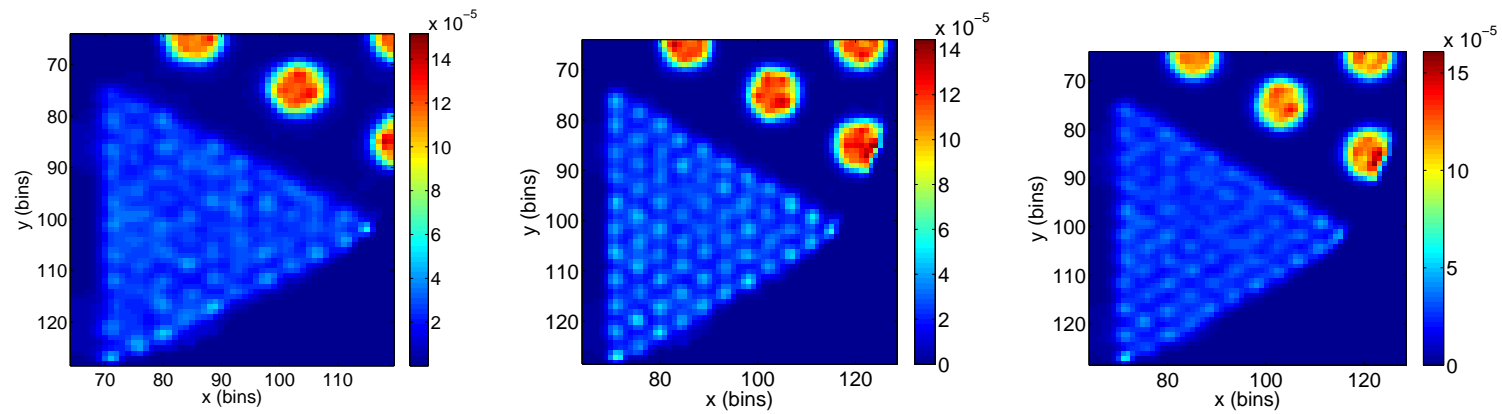

Figure 7.23: Images of $1.2 \mathrm{~mm}$ rods that have been corrected for the positron range with the 3D ML-EM algorithm positron range correction and post blurred for a $0.7 \mathrm{~mm}$ Gaussian system resolution: (left) $0 \mathrm{~T}$, (middle) $7 \mathrm{~T}$ with three orientations, and (left) a $7 \mathrm{~T}$ magnetic field in the axial z-direction. 

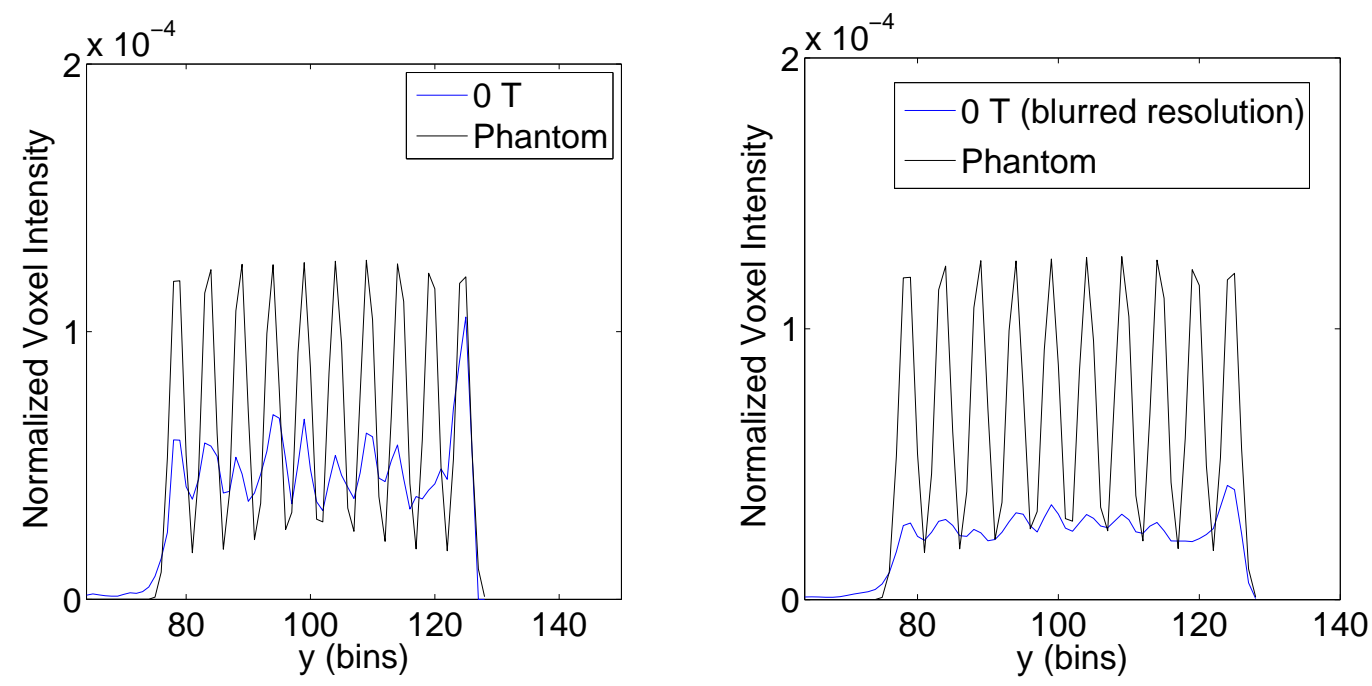

Figure 7.24: Slices through the $1.2 \mathrm{~mm}$ rods of the positron range corrected images at $0 \mathrm{~T}$ without blurring for the $0.7 \mathrm{~mm}$ FWHM system resolution (left) and with blurring for the system resolution (right).
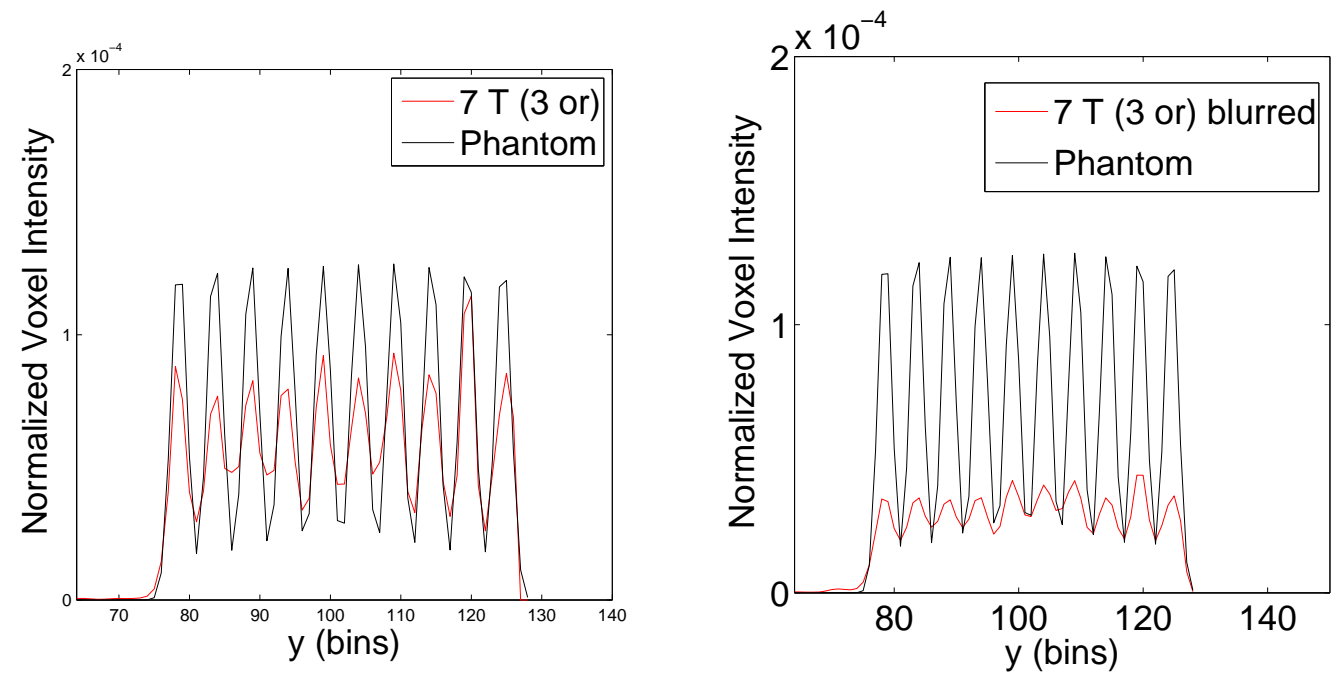

Figure 7.25: Slices through the $1.2 \mathrm{~mm}$ rods of the positron range corrected images at $7 \mathrm{~T}$ (three magnetic field orientations) without blurring for the $0.7 \mathrm{~mm} \mathrm{FWHM}$ system resolution (left) and with blurring for the system resolution (right). 

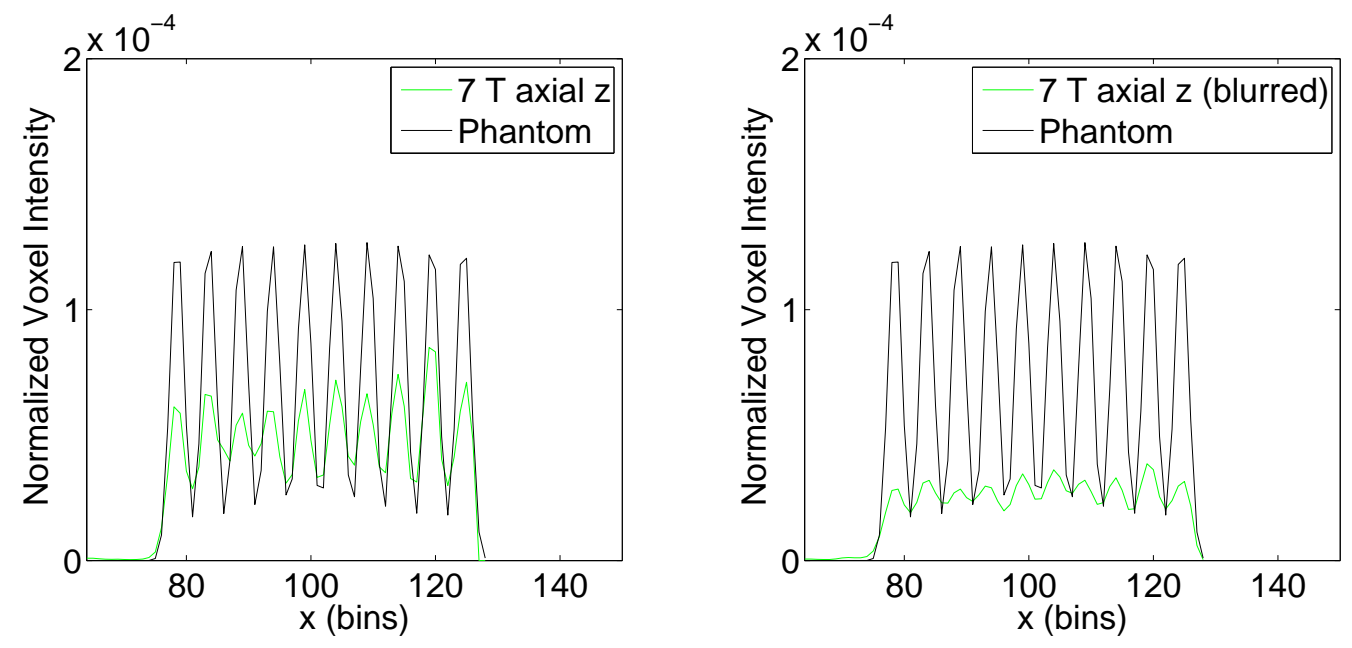

Figure 7.26: Slices through the $1.2 \mathrm{~mm}$ rods of the positron range corrected images at $7 \mathrm{~T}$ (axial z-direction) without blurring for $0.7 \mathrm{~mm}$ FWHM the system resolution (left) and with blurring for the system resolution (right).

Thus far it has been shown that the positron range can be completely removed for simple source configurations using either $0 \mathrm{~T}$ or $7 \mathrm{~T}$ data, but for more complex sources there is a slight advantage to collecting data at $7 \mathrm{~T}$. However, when other sources of image blur, such as the system resolution, are taken into account this advantage is not apparent. Visually examining images and slices can only qualitatively display the benefits or drawbacks of using one imaging modality over another. The following section seeks to quantify the imaging performance of data collected in a strong magnetic field compared to data collected at $0 \mathrm{~T}$ by investigates the tradeoff between the noise and the resolution for PET images reconstructed with a 3D ML-EM positron range correction algorithm. 


\subsection{Resolution versus Noise Study}

Thus far all of the positron sources have been localized in a background devoid of positron emitters. In an actual PET environment the radioactive tracer will not only congregate in the target cells. Instead, there will be a constant background source of the radioactive tracer that has not and will not be processed by the target cells. The real challenge in PET is to resolve the difference in the intensity of the number of annihilation pairs emitted from the target cells in the presence of a background distribution. In the case of detecting cancer, the more cancerous cells congregated in a location will result in a larger buildup of the radioactive tracer above the activity level of the radioisotope that has not been processed by the cancerous cells (this is the background source). However, as the number of cancer cells decrease, so will the difference between the number of photon pairs emitted from the target cells and the background distribution. At some point the photon pairs from the cancer cells will be concealed by deviations in the background distribution due to the Poisson distribution of annihilation events. High resolution PET detectors allow us to probe smaller and smaller volumes which translates into the ability to detect the onset of cancer sooner. However, there is a draw back to operating at high resolutions when the activity of the source is on the same order (or less) as the background distribution. As previously discussed, the distribution of the detected LORs is Poisson. As the resolution of the system increases, variations in the number of detected events along each LOR will become more apparent. In fact, as the resolution continues to improve large differences in the Poisson distributed background will be reconstructed as actual sources. The ideal situation is to have a high resolution PET system that can detect very small 
sources while also minimizing the variation in the Poisson distributed events. This variation in events is termed the noise for the remainder of this study.

To illustrate the above argument, consider a cylindrical background distribution ${ }^{6}$ filled with a ${ }^{68} \mathrm{Ga}$ positron range distribution in water at $0 \mathrm{~T}$. Within the constant background a ${ }^{68} \mathrm{Ga}$ point source possessing a tenth of the activity of the background distribution is superimposed onto the background distribution within a single voxel. A 3D ML-EM positron range correction algorithm is used to reconstruct the data simulated from this source distribution for two cases. One, the constant background is not varied by the Poisson nature of the PET data. Two, the background distribution of events are varied by Poisson statistics. The same number of events are used in both reconstructions $\left(10^{8}\right)$. Figs. 7.27 through 7.29 are both reconstructed with the 3D ML-EM positron range correction algorithm using the ${ }^{68} \mathrm{Ga} 0 \mathrm{~T}$ positron range distribution in water. In these figures the final images/projections are the results after 1000 (left) and 6000 (right) iterations of the reconstruction algorithm, respectively. For PET systems with sufficient resolutions the small point source can be resolved within the background distribution as shown in the images contained in Fig. 7.27 (a black circle indicates the location of the embedded source). However, when the background is varied to take into account the Poisson nature of the PET data the small point source can no longer be resolved as can be seen in the images in Fig. 7.28 (the red circle indicates the location of the embedded source, which can be resolved in the noiseless background, but is completely obscured by the variation of the background.). Figure 7.29 contains the slice along the x-direction through the source position for both the constant noiseless background and in the presence of Poisson distributed

\footnotetext{
${ }^{6}$ The source distribution used in this example is described in detail in section 7.5.2.
} 

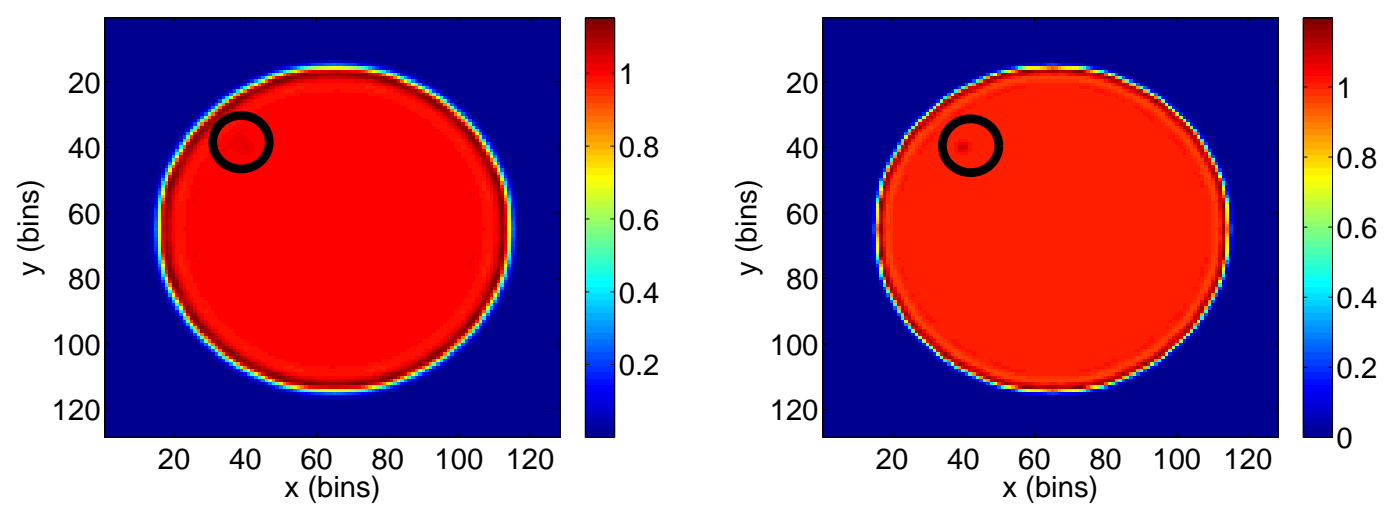

Figure 7.27: The 3D ML-EM positron range corrected image of a ${ }^{68} \mathrm{Ga}$ point source possessing $1 / 10$ of the activity of the ${ }^{68} \mathrm{Ga}$ constant background distribution at $0 \mathrm{~T}$ (in water) in the absence of Poisson distributed events (left) at 1000 iterations and (right) at 6000 iterations. The source is located in voxel $(40,40)$, and is surrounded by the the black ring in the images.

events using 1000 (left) and 6000 (right) iterations of the reconstruction algorithm. The different number of iteration numbers is used to illustrate that images with higher resolution will possess larger variation in the reconstructed voxel intensities. This plot also demonstrates that the variation in the voxel intensity (noise) is greater for higher resolution images.

A technique used to quantify and compare the image quality of various imaging modalities (0 T, $7 \mathrm{~T}$ axial, $7 \mathrm{~T} 3$ orientations) is used to create plots relating the noise and the resolution. The noise, in this context, is the Poisson variation in the number of detected events from a particular voxel, while the resolution is the width of a small test source, or perturbation, inserted into a constant background distribution that is not varied by Poisson statistics. Figure 7.30 is a generic resolution-noise graph that is used to illustrate this idea. The resolution is calculated by extracting the width of a reconstructed test object superimposed in a single voxel of the background 

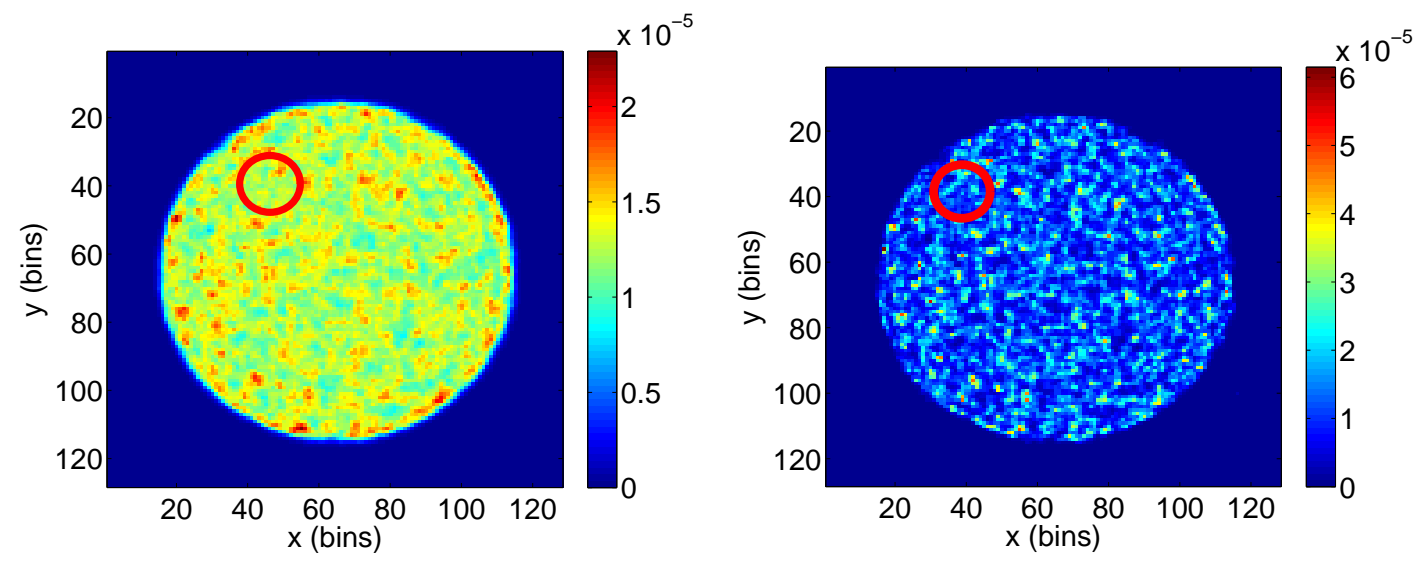

Figure 7.28: The 3D ML-EM positron range corrected image of a ${ }^{68} \mathrm{Ga}$ point source possessing $1 / 10(1 / 100$ is used later in the study - 1/10 is used here such that the source is more easily identified) of the activity of the ${ }^{68} \mathrm{Ga}$ constant background distribution at $0 \mathrm{~T}$ (in water) with Poisson distributed events (left) at 1000 iterations and (right) at 6000 iterations. The source is located in voxel $(40,40)$, and is surrounded by the red ring in the images. For both cases the source is obscured by the variation in the background events. This plot also demonstrates that the variation in the noise increases as the resolution increases.

distribution. The noise contribution of the graph is found by reconstructing $N$ noise background realizations and then extract the voxel intensity for each realization from the same voxel location that contained the perturbative source for the resolution image, and finally calculate the standard deviation of the $N$ voxel intensity values. Section 7.5.1 addresses the generation of a noise-resolution curve in more detail.

The noise resolution graph is used to compare two or more systems by asking the question: at a given resolution, which system has the lowest noise? This question is easily answered by observing which resolution-noise curve is lower on the graph. The lower curve will yield images with less variation in the noise at a given resolution, i.e. cleaner images. The general feature of all noise-resolution curves is that there is a trade-off between the noise and the resolution (higher resolution images will reveal 

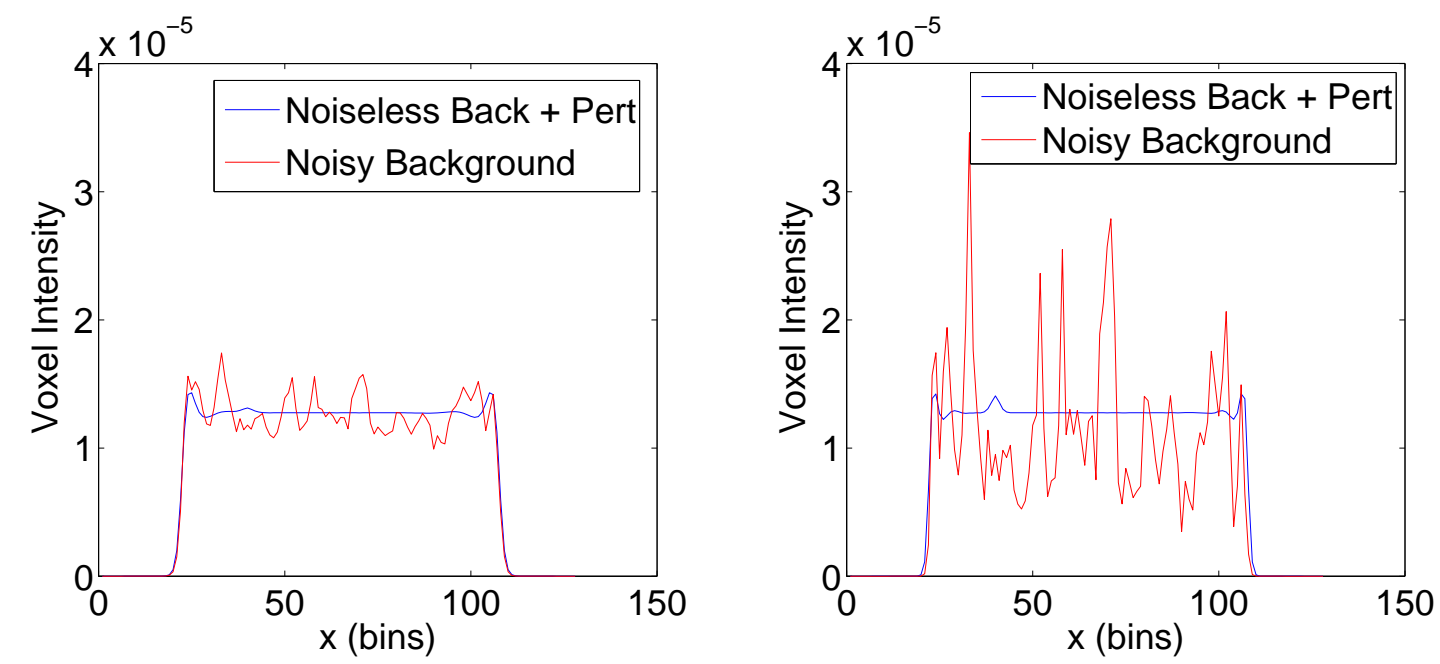

Figure 7.29: A projection along the bin passing through the ${ }^{68} \mathrm{Ga}$ point source in the constant ${ }^{68} \mathrm{Ga}$ background distribution from the images in Fig. 7.27 without (blue) and with (red) Poisson distributed events. The projection on the left is for 1000 iterations and the image on the right is for 6000 iterations of the 3D ML-EM positron range correction algorithm. For both cases the source is obscured by the variation in the background events. This plot also demonstrates that the variation in the noise increases as the resolution increases. 


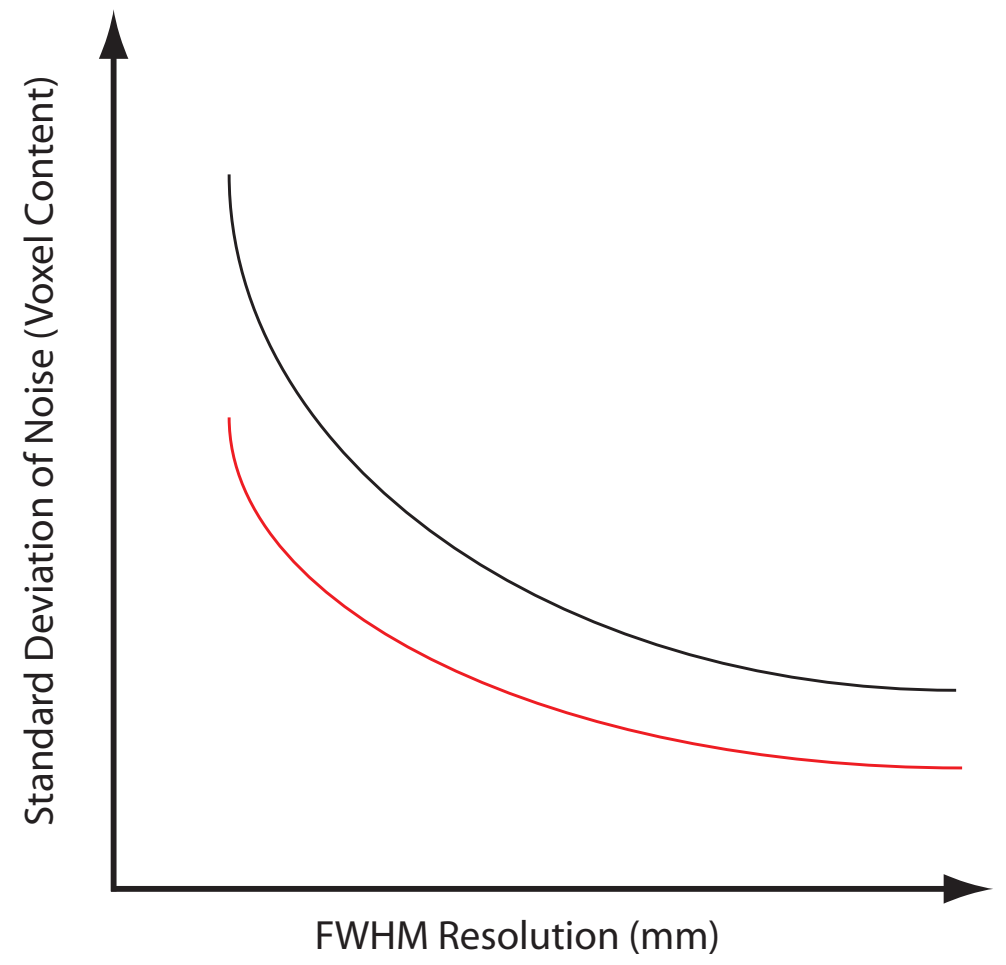

Figure 7.30: Example noise versus resolution plot that displays the characteristic trade-off between the resolution and the noise for two hypothetical systems. The system with the red resolution noise curve will yield images with lower noise for any given system resolution. 
more noise while lower resolution images will further blur out deviations in the Poisson distributed events resulting in less noise - as shown by the projections in Fig. 7.29.).

This study only includes reconstructed voxel noise due to the Poisson nature of the PET data and does not include noise due to random or scattered coincidence events.

\subsubsection{An algorithm for creating noise-resolution trade-off curves}

A "brute force" algorithm (as described by Fessler and Rogers [60]) is used to generated the resolution-noise trade-off curves. The purpose of this algorithm is to determine the resolution response (or point spread function - PSF) of a low activity, single voxel source embedded in a constant background distribution and to determine the variance in the noise for the same voxel in the presence of the Poisson statistics of the measured LORs. The same voxel location that contains the perturbative source used to determine the PSF is also used in the $N$ noisy realizations to eliminate variations in the noise that may be location sensitive. Variations in the noise due to the geometric location of the test voxel should not be an issue because the voxel of interest is positioned far from the edge of a constant, uniform background. It is only near the edge where reconstruction artifacts will appear.

The following three part algorithm describes the steps used to generate a noiseresolution curve.

- Step 1: Generate $N$ noisy background realizations (without a perturbative source). A 3D background object $\boldsymbol{\lambda}$, blurred by the desired positron range distribution, is forward projected into sinogram space via a Radon transform creating a stack of $2 \mathrm{D}$ sinograms. Each sinogram pixel is assigned a random 
Poisson value as described in section 7.2. A reconstruction algorithm creates a image that attempts to matches the data contained in the sinogram stack. $N$ realizations are performed with each realization possessing a different random set of Poisson distributed data. These $N$ images are later used (in step 3) to determine the standard deviation in the reconstructed noise.

\section{- Step 2: Generate the system response (PSF) in a constant, noiseless} background. Independent of the $N$ noisy images, two noiseless images that have not been varied by Poisson statistics are reconstructed to produce the system response (PSF). The first image is a reconstruction of the noiseless, background object. The second image is identical to the first but with a small perturbative source placed at a known, single voxel location. In this study, the perturbation is $1 / 100$ the intensity of a background voxels. Both the noiseless background with and without the perturbation are reconstructed with the same reconstruction algorithm that was used to create the images of the noisy data. The image containing the PSF is found by taking the difference between the two images on a voxel-by-voxel basis. The PSF is a measure of the current image resolution (at a particular iteration) due to the reconstruction algorithm (until the images have fully converged, more iterations lead to a higher resolution PSF). Both the noiseless data with the perturbation and the noisy Poisson distributed data converge at the same rate, thus the PSF is used as a probe to determine the resolution of the noisy data.

- Step 3: Blur the images in steps 1 and 2 by the desired system resolution and use the resulting images to calculate the noise-resolution 
curve The resolution-noise curve is created by blurring both the $N$ noisy realizations and the PSF by the same desired system resolution. The standard deviation of the voxel of interest is calculated from the $N$ realizations of the noisy Poisson distributed data (this gives the noise value of each point on the curve). The resolution portion of the curve is extracted from the FWHM of the convolution of the PSF with the desired system resolution. Both the noise and resolution are plotted for convolutions with various detector resolutions. In this study the images are blurred by 3D Gaussian system resolutions with FWHM resolution of $0.4 \mathrm{~mm}, 0.8 \mathrm{~mm}, 1.2 \mathrm{~mm}, 1.6 \mathrm{~mm}$, and $2.0 \mathrm{~mm}$.

Ideally, each image created with this algorithm would be fully converged, i.e. the positron range has been completely corrected for. For the case of fully converged images, the system response calculated is step 2 would approach a delta-like function. Also, the variation in the noise due to the Poisson statistics would be at a maximum. Extracting the width of the fully converged PSF and calculating the standard deviation of the noise from the $N$ realizations would give a point on the noise-resolution curve that yields the best resolution, but also has the highest noise. Using the fully converged PSF and the $N$ realizations possessing the maximum amount of noise, the following question can be easily answered: what is the variation in the noise for any given system resolution. To answer this question both the fully converged PSF and the $N$ maximum noisy realizations are blurred by the desired system resolution. Blurring the PSF with the detector resolution will simply return the detector resolution if the PSF is truly a delta-like function. The variation in the noise is blurred with the same detector resolution as the PSF, which smoothes the variations in the noise. The width of the blurred PSF yields the resolution portion of the point, while the 
standard deviation is calculated from the single voxel intensities extracted from the $N$ blurred realizations. In this manner, the noise-resolution curve can be calculated by blurring both the ideal PSF and the $N$ noisy realizations for any desired system resolution.

\subsubsection{Source geometry}

The geometry of the background source used to study the noise-resolution properties of our systems is a cylinder of uniform activity, 100 bins (40 mm) in diameter and 10 bins $(4 \mathrm{~mm})$ in height (each of the ten $0.4 \mathrm{~mm}$ thick bins that constitute the height of the cylinder correspond to a different 2D image plane). The background distribution is blurred by the positron range using either the $0 \mathrm{~T}$ or $7 \mathrm{~T}$ magnetic field directed in the axial direction, or in three independent, orthogonal orientations. Figure 7.31 displays the central slice of the background without any blurring (left) and blurred with the $0 \mathrm{~T}^{68} \mathrm{Ga}$ positron range in water (right). Figure 7.32 and 7.33 show the background distribution blurred for the $7 \mathrm{~T}$ positron range oriented along the $\mathrm{x}, \mathrm{y}$, and $\mathrm{z}$ directions, respectively. Note the widening of the positron range on the edge of the background due to the magnetic fields directed along the $\mathrm{x}$ and $\mathrm{y}$ axes (Fig. 7.32). An example of a sinogram created from the central $0 \mathrm{~T}$ background slice shown in Fig. 7.31 without and with Poisson distributed events is shown in Fig. 7.34 on the left and right, respectively. Figure 7.35 shows examples of the central slice of the reconstruction images of the background distribution at 1000 iterations using the 3D ML-EM positron range correction algorithm at $0 \mathrm{~T}$ (left) and $7 \mathrm{~T}$ using 3 orthogonal magnetic field orientations (right). 

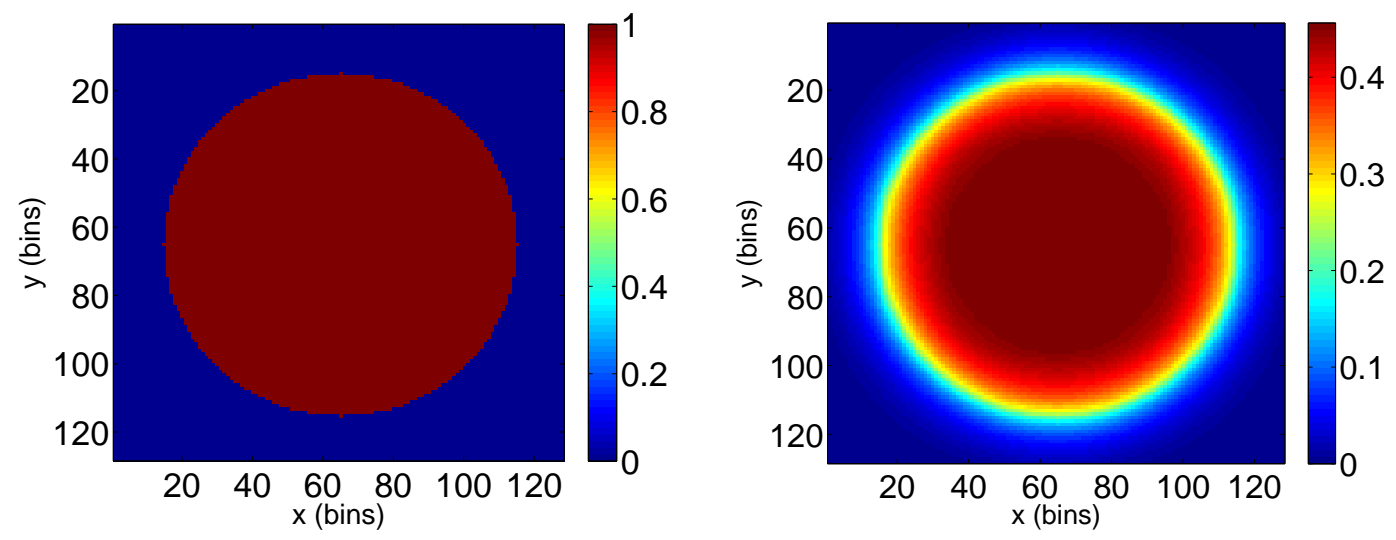

Figure 7.31: Left: Central slice of the uniform background source. Right: Central slice of the uniform background source blurred for the $0 \mathrm{~T}^{68} \mathrm{Ga}$ positron range in water.
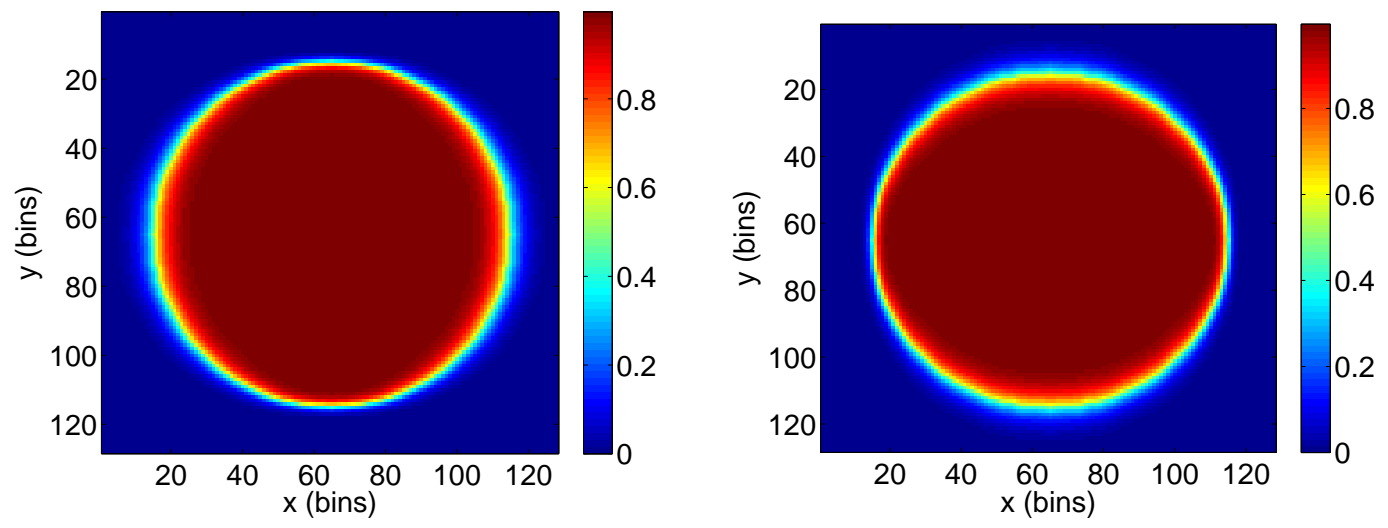

Figure 7.32: Background blurred with two orientations of the $7 \mathrm{~T}$ magnetic field. The magnetic field is oriented in the positive y-direction for the image on the left, and in the positive $\mathrm{x}$-direction for the image on the right. Note the blurring of the background distribution in the directions transverse to the magnetic field vector. 


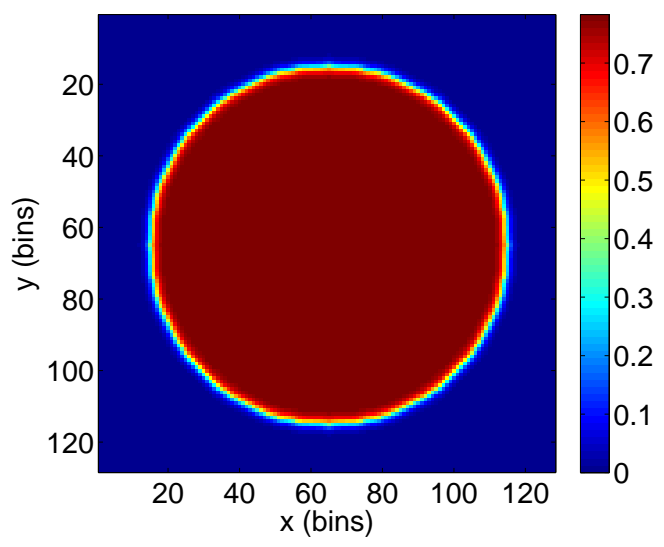

Figure 7.33: Background blurred with the ${ }^{68} \mathrm{Ga}$ positron range distribution in water with a $7 \mathrm{~T}$ magnetic field in the axial z-direction. Note that the blurring along the edges of the source have been significantly reduced.
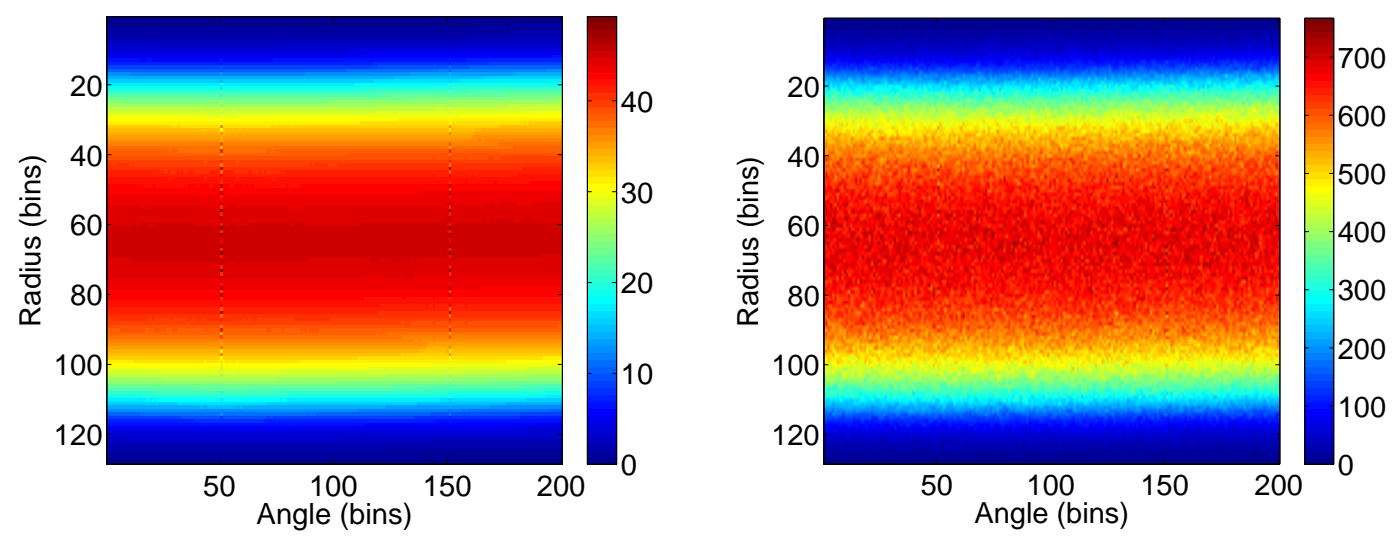

Figure 7.34: Sinogram of the $0 \mathrm{~T}^{68} \mathrm{Ga}$ positron range blurred background without (left) and with (right) Poisson distributed events applied. 

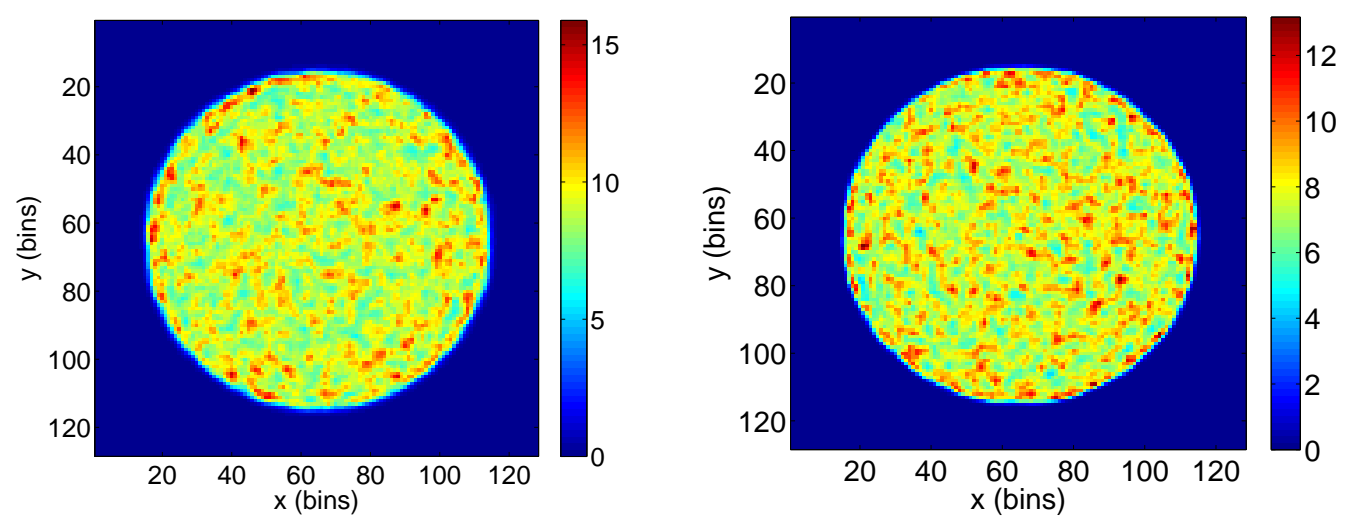

Figure 7.35: Reconstructed images with $10^{8}$ Poisson distributed events for $0 \mathrm{~T}$ and 7 T (3 orientations) simulated data for 1000 iterations of the 3D ML-EM positron range correction algorithm.

\subsubsection{Creating the PSFs}

The PSFs are used as the probe that tells us the resolution that has been recovered in the noisy images. Ideally, all of the PSFs would have identical shapes such that any variation in the noise is due to the imaging mode (i.e. the case we are using) and not due to unintentional blurring due to differences in the PSF widths. However, achieving identical PSFs in not possible using the current 3D ML-EM positron range correction algorithm. The larger the difference between the PSF shapes, the larger the uncertainty in the recovered noise. To see this, recall the plots shown in Fig. 7.29. In these plot, two different PSFs are created by running the 3D ML-EM reconstruction algorithm for different numbers of iterations. The plot on the left is made using 1000 iterations while the plot on the right uses 6000 iterations. Running the reconstruction algorithm for additional iterations reveals more variation in the Poisson distributed events. Analogously, the wider PSF obscures variations in the Poisson distributed 


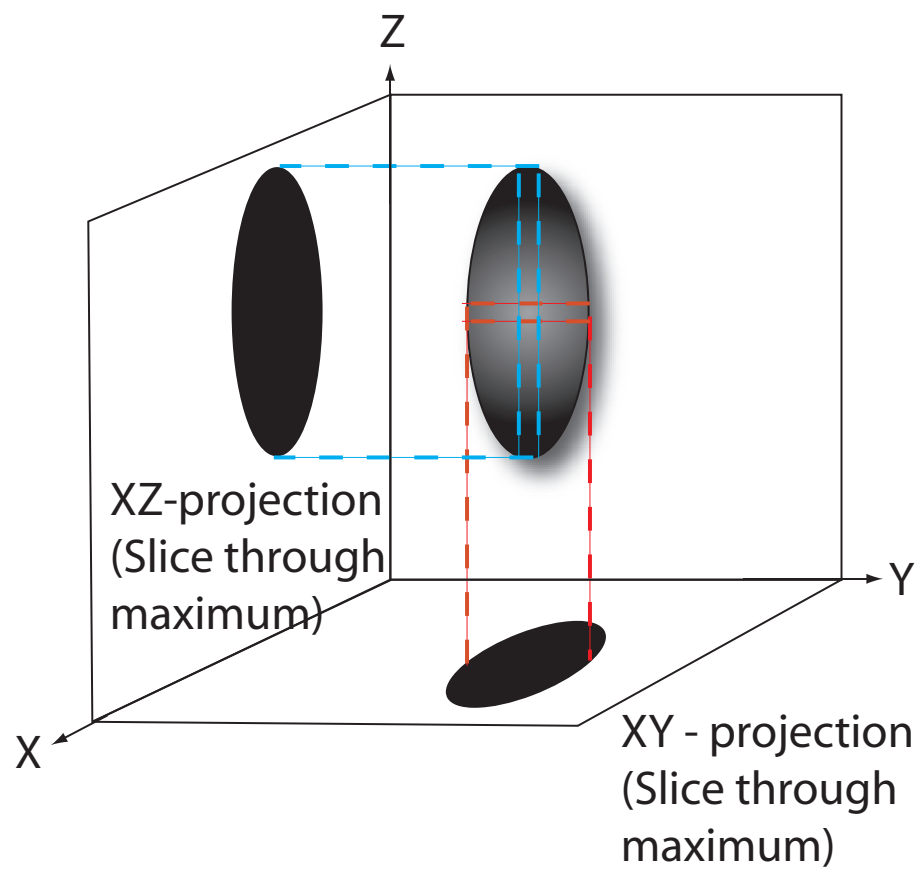

Figure 7.36: A graphical representation of the projection slices shown in this section.

events, returning results that indicate the system has less variation in the noise than it actually does. In this chapter we seek to minimize the difference between the PSFs in order to get the best estimate of the image performance between the three different data collection cases. This section outlines the steps that have been followed to create PSFs that resemble each other as closely as possible using the current reconstruction algorithm, and outlines the differences in the PSFs and their possible effects on the final results. This section gives an initial comparison between the various data collection cases and outlines avenues for future research that will yield more accurate results.

As discussed in section 7.5.1, a small perturbative source is inserted into the noiseless background in order to determine the PSF of the system (either $0 \mathrm{~T}, 7 \mathrm{~T}$ 
(axial), or $7 \mathrm{~T}$ (3 orientations)). The magnitude of the perturbation used in this study is 0.01 , which is one percent of the background intensity. The PSFs are displayed in this section by cross-sections ( $0.4 \mathrm{~mm}$ thick, or a single voxel thickness) through the central maxima as illustrated in Fig. 7.36. The central ellipse represents the three dimensional PSF and the two ellipses on the xy and xz plane are projections of slices that bisect the maximum of the PSF.

The following set of images are not the final PSFs used to create the noiseresolution curves. Instead, these PSFs are used to illustrate some of the difficulties in achieving PSFs of similar shape. The following plots compare the $0 \mathrm{~T}$ and $7 \mathrm{~T}$ (3 orientation) PSFs created using 1000 iterations of the 3D ML-EM positron range correction algorithm for both cases. Figure 7.37 contains the xy-plane passing through the maximum of the $0 \mathrm{~T}$ (left) and $7 \mathrm{~T}$ (3 orientations) (right) PSFs. The range of the plot has been zoomed into the region containing the perturbation. Figure 7.38 contains the zoomed in images of the xz plane of the PSFs. Fig. 7.39 are slices through the center of the PSFs for the $0 \mathrm{~T}$ and $7 \mathrm{~T}$ data in the $\mathrm{x}$ and $\mathrm{z}$ direction after 1000 iterations. It can be seen that width of the PSFs in the axial, z-direction, is tighter than the planar, $\mathrm{x}$-direction for both the $0 \mathrm{~T}$ and $7 \mathrm{~T}$ PSFs. This is likely due to the cylindrical symmetry of the setup. These figures show that there are major differences between the two PSFs. The maximum height of the $0 \mathrm{~T}$ PSF demonstrates that it is not converging as quickly as the $7 \mathrm{~T}$ (3 orientation) PSF. This means that less variations in the noise will be revealed. Also, the $0 \mathrm{~T}$ PSF is symmetric in the 
xy-plane while the $7 \mathrm{~T}$ (3 orientation) PSF possess a shape resembling a $\operatorname{cross}^{7}$. Depending on the severity of the difference between the two PSF shapes, various noise properties may be masked.
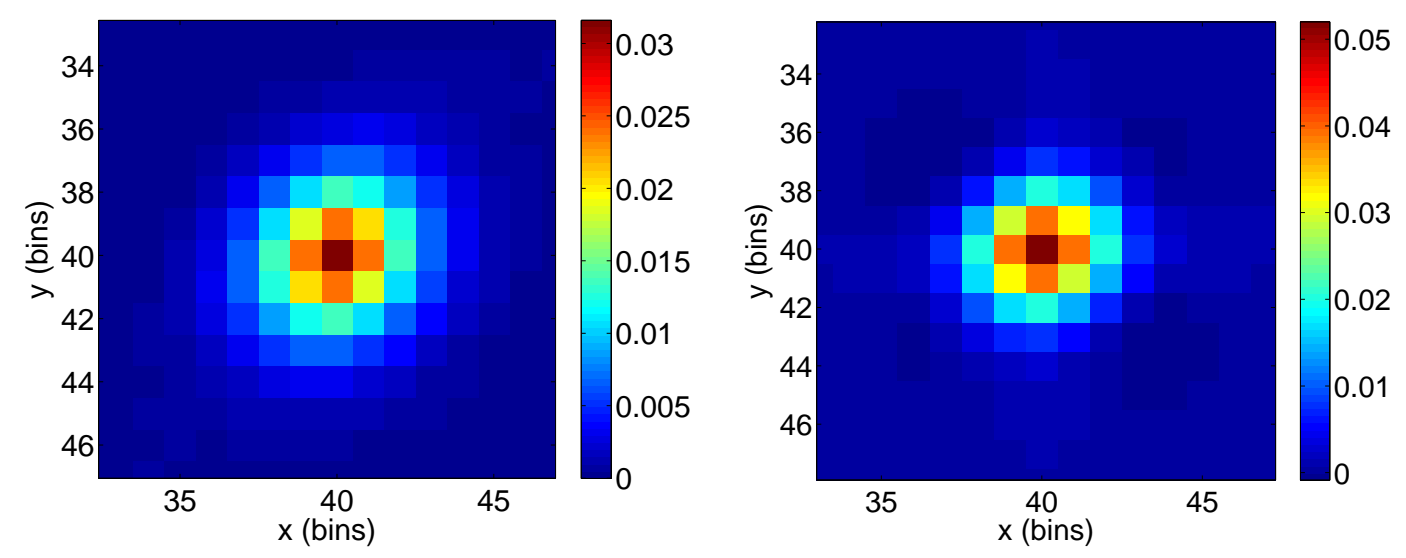

Figure 7.37: $0 \mathrm{~T}$ and $7 \mathrm{~T}$ (3 orientations) PSFs in the xy-plane. Note that the positron range correction is having difficulty in this environment. In particular note the crossing artifact in the $7 \mathrm{~T}$ data suggesting that the algorithm is slow to remove the positron range.

It has been shown that both the $0 \mathrm{~T}$ and $7 \mathrm{~T}$ (3 orientation) PSFs are far from convergence (0.4 mm FWHM for full positron range removal) at 1000 iterations of the 3D ML-EM positron range correction algorithm. In order to study the rate of convergence of both PSFs the FWHM resolution in the x-direction versus iteration number was studied. The results are shown in Fig. 7.40 up to 6000 iterations. This plot shows that both the $0 \mathrm{~T}$ and $7 \mathrm{~T}$ (3 orientations) PSFs are both converging slowly and at the same rate. The $7 \mathrm{~T}$ PSFs possess a better resolution than the $0 \mathrm{~T}$ PSFs when the same number of iterations are used. Thus, in order to have PSFs of

${ }^{7}$ This may be difficult to see depending on the quality of printer; however, the difference is clear when viewing the pdf on a computer monitor. 

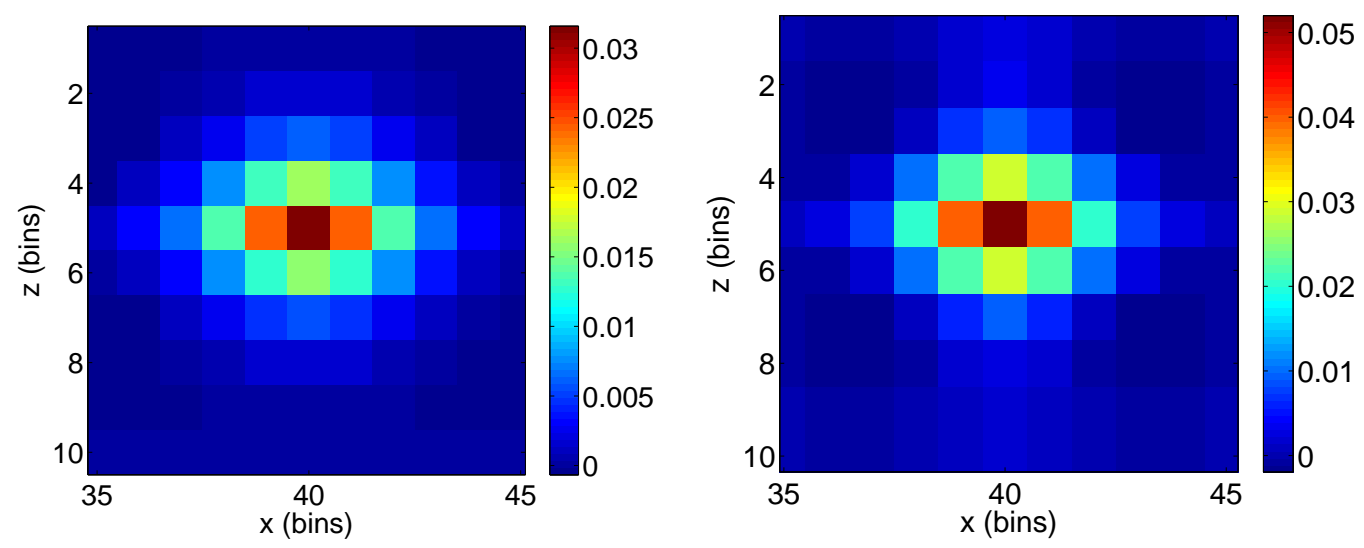

Figure 7.38: $0 \mathrm{~T}$ and $7 \mathrm{~T}$ (3 orientations) PSFs in the xz-plane. Note that the positron range correction is having difficulty in this environment. In particular note the crossing artifact in the $7 \mathrm{~T}$ data suggesting that the algorithm is slow to remove the positron range.
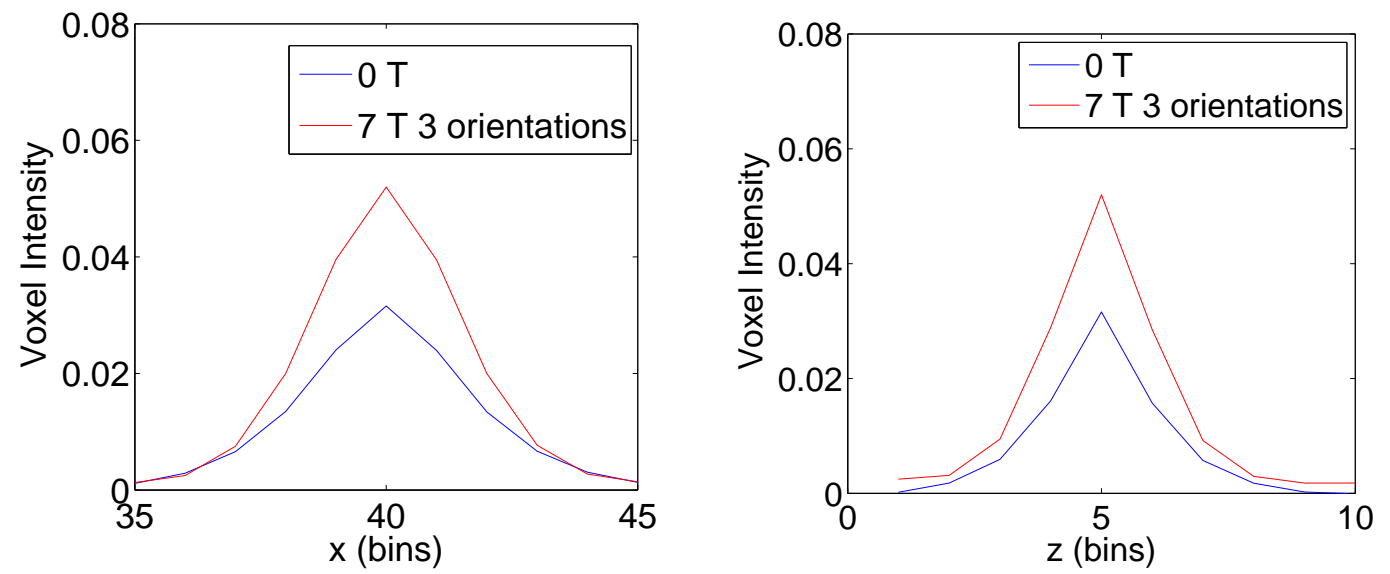

Figure 7.39: Slice through maximum in $\mathrm{x}$ and $\mathrm{z}$ direction for $0 \mathrm{~T}$ and $7 \mathrm{~T}$ (3 orientation) data. The widths for the x-slices are: $\mathrm{FWHM}_{0 T}=1.50 \mathrm{~mm} \mathrm{FWTM}_{0 T}=3.19$ $\mathrm{mm}, \mathrm{FWHM}_{7 T}=1.40 \mathrm{~mm}, \mathrm{FWTM}_{7 T}=2.84 \mathrm{~mm}$. The width for the z-slices are: $\mathrm{FWHM}_{0 T}=0.87 \mathrm{~mm}, \mathrm{FWTM}_{0 T}=2.13 \mathrm{~mm}, \mathrm{FWHM}_{7 T}=0.96 \mathrm{~mm}, \mathrm{FWTM}_{7 T}=1.58$ mm. 
similar widths more iterations are need for the $0 \mathrm{~T}$ PSF than the $7 \mathrm{~T}$ PSF. A plot of the 0 T PSF's FWHM resolution in the x-direction versus iteration number is shown in Fig. 7.41 up to 12,000 iterations. This plot shows that the PSF is converging at a nearly constant rate. Assuming the same rate of convergence seen in the figure it would take approximately 30000 iterations to fully remove the positron range, or 1.25 days of processing per realization for the $0 \mathrm{~T}$ and 3.75 days per realization for the 7 T data running on eight $2.5 \mathrm{GHz}$ processors. For 25 noisy realizations, this translates into 31 and 94 days of processing for the $0 \mathrm{~T}$ and $7 \mathrm{~T}$ data, respectively.

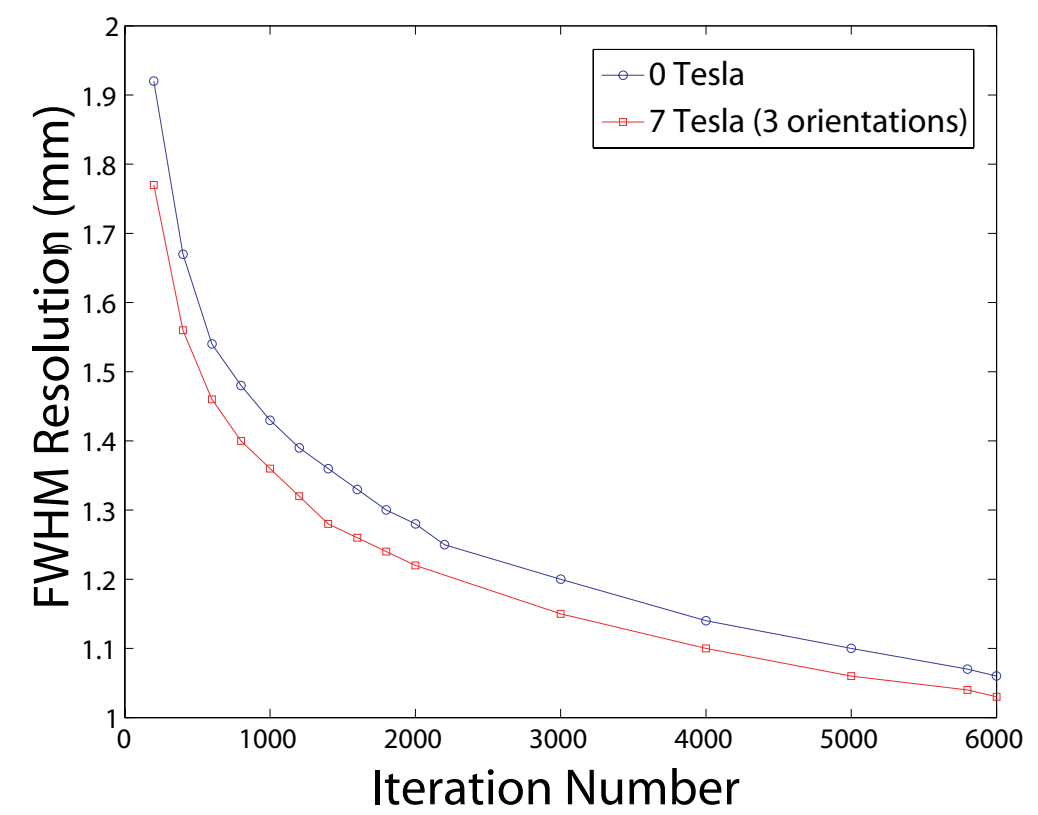

Figure 7.40: FWHM resolution along the x-axis versus iteration for a small perturbative source in a constant background at both $0 \mathrm{~T}$ and $7 \mathrm{~T}$ using three orthogonal magnetic field orientations.

It is clear that a reconstruction algorithm with faster convergence properties is needed. For the current study it was decided that the systems will be compared 


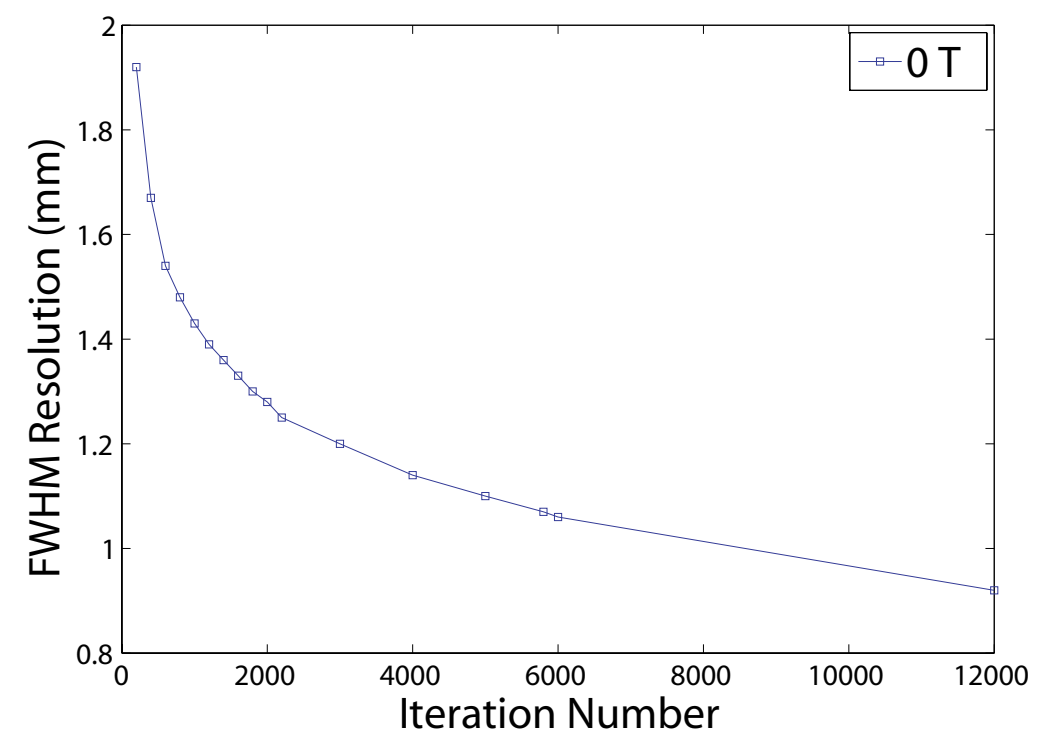

Figure 7.41: Resolution versus iteration number for 0 T ML-EM 3D positron range correction algorithm.

for PSFs that are similar to the $0 \mathrm{~T}$ PSF at iteration 6000 . This PSF is reasonably converged to extract meaningful noise-resolution plots while still being able to process the data in a reasonable amount of time. The challenge is to find PSFs for the $7 \mathrm{~T}$ (axial) and the $7 \mathrm{~T}$ (3 orientations) that closely match the $0 \mathrm{~T}$ PSF. This study will demonstrate the $7 \mathrm{~T}$ (3 orientation) PSF can be matched more closely than the $7 \mathrm{~T}$ (axial) PSF and has a result will yield a more confident result.

The PSFs that most closely match the 0 T PSF was found by examining the shape of the projection plots along the $\mathrm{x}, \mathrm{y}$, and $\mathrm{z}$ axis for various iteration numbers and finding a criteria that best describes the closeness of the fit. Figure 7.42 shows the $0 \mathrm{~T}$ PSF along with the three closest matching $7 \mathrm{~T}$ (3 orientation) PSFs for the $\mathrm{x}$ and $\mathrm{z}$ slice through the maximum (the $\mathrm{y}$ slice is identical to the $\mathrm{x}$ slice for all of the cases in this study, and as a result is not shown). It can be seen in this plot that 
major differences between the the $0 \mathrm{~T}$ PSF at iteration 6000 and the 7 T PSFs at various iterations is the height of the peak. This observation is more apparent in (Fig 7.44 which is a zoomed in image of the peak along the x-slice). Due to the fact that the edges of the distribution are nearly identical for all three closest matching PSFs, the height of the each peak is the determining factor for choosing the closest PSF. From the figure is it clear that iteration 3600 for the $7 \mathrm{~T}$ (3 orientation) plot is the closest match. Also shown in Fig. 7.42 is the projection of the PSFs along the z-slice through the maximum. This plot demonstrates that the PSF in the z-direction is not as close of a match to the $\mathrm{x}$ and $\mathrm{y}$ slices. This difference is unavoidable using the current reconstruction algorithm. If the width of the z-slices is made to match the $0 \mathrm{~T}$ PSF at iteration 6000, then the $7 \mathrm{~T}$ (3 orientation) PSF in $\mathrm{x}$ and $\mathrm{y}$ would be much tighter, resulting in a poor match in the $\mathrm{x}$ and $\mathrm{y}$ plane. This small deviation in the z-slice will still yield comparable results.

The $7 \mathrm{~T}$ (axial) PSFs do not match as well to the $0 \mathrm{~T}$ PSFs as do the $7 \mathrm{~T}$ (3 orientation) PSFs. This is due to the asymmetry introduced by only using one magnetic field orientation. Fig. 7.43 shows $7 \mathrm{~T}$ axial PSFs that are the closest matches of the $\mathrm{x}$ and $\mathrm{z}$ slices to the $0 \mathrm{~T}$ PSF at iteration 6000 . From these plots it is seen that iteration 3200 of the reconstruction algorithm yields the closest match to the 0 T PSF.

A comparison of the PSF widths for each case for the $\mathrm{x}$ and $\mathrm{z}$ projections are shown in Figure 7.45. These plots demonstrate that the $0 \mathrm{~T}$ and $7 \mathrm{~T}$ (3 orientation) PSFs are in reasonably good agreement. However, the $7 \mathrm{~T}$ (axial) PSF is significantly wider than the other two PSFs along the z-axis. The wider PSF will reduce deviations in the noise, leading to lower points on the noise-resolution curve. It is expected that 
the actual performance of the $7 \mathrm{~T}$ (axial) system will be noisier if a better PSF match could be found. Again, a better reconstruction algorithm needs to be developed to address these issues. Table 7.2 lists the FWHM and FWTM values of the PSFs shown in Fig. 7.45 for the projections along the $\mathrm{x}$ and $\mathrm{z}$, respectively.
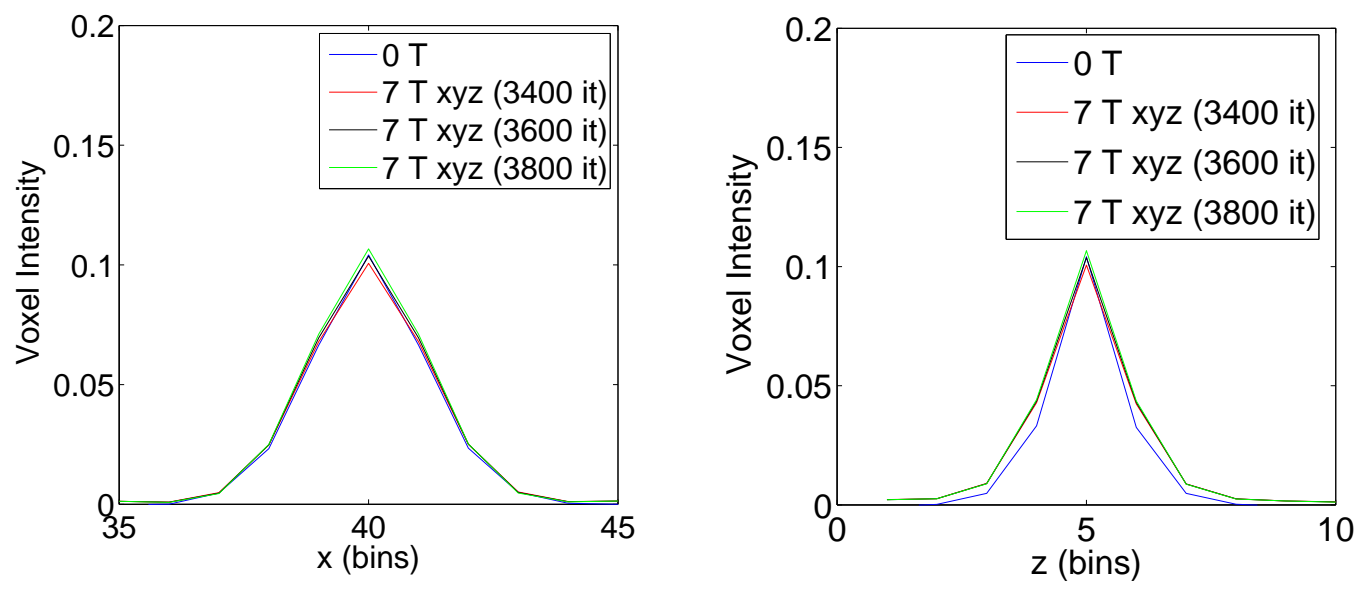

Figure 7.42: The $\mathrm{x}$ and $\mathrm{z}$ projections through the maxima of the $0 \mathrm{~T}$ and $7 \mathrm{~T}(3$ orientation) PSFs reconstructed with 3400, 3600, and 3800 iteration. The purpose of this plot is to show that all three iterations numbers are close matches to the $0 \mathrm{~T}$ PSF. Fig. 7.44 shows why iteration 3600 iterations (black line) of the 3D ML-EM positron range correction algorithm yields the closest match of the $7 \mathrm{~T}$ (3 orientations) PSF to the $0 \mathrm{~T}$ PSF.

Differences also exist between the voxel content of the PSFs from voxels located off axis. Figure 7.46 through 7.49 are the reconstructed images along the planes passing through the center of each PSF as illustrated in Fig. 7.36. Figure 7.46 are the projections of the reconstructed images along the xy-plane for the three seperate cases. The full FOV is shown in this plots in order to display the relative size of each point spread function. It is not until you zoom in on the PSFs that the subtle differences become apparent. Figures 7.47 and 7.49 are zoomed in images of the 

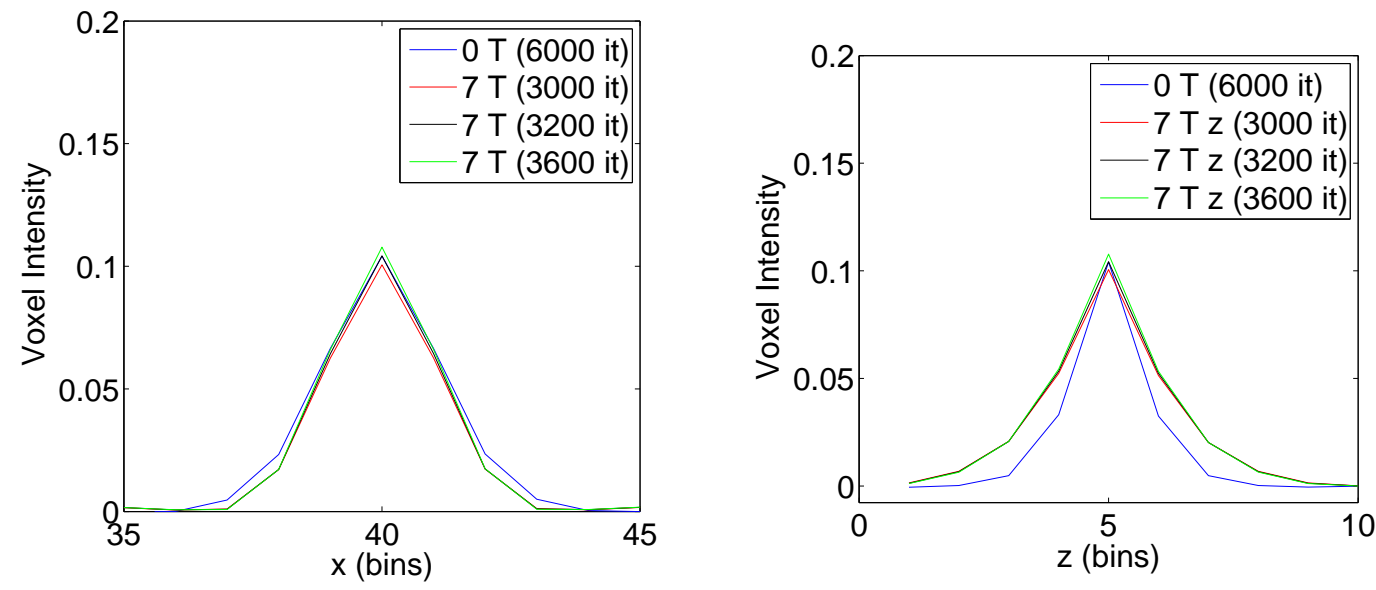

Figure 7.43: The $\mathrm{x}$ and $\mathrm{z}$ projections through the maxima of the $0 \mathrm{~T}$ and $7 \mathrm{~T}$ (axial) PSFs reconstructed with 3200, 3400, and 3600 iterations. The purpose of this plot is to show that all three iterations numbers are the closest match to the $0 \mathrm{~T} \mathrm{PSF}$. Fig. 7.44 shows why iteration 3200 iterations (black line) of the 3D ML-EM positron range correction algorithm yields the closest match of the $7 \mathrm{~T}$ (axial) PSF to the 0 T PSF.
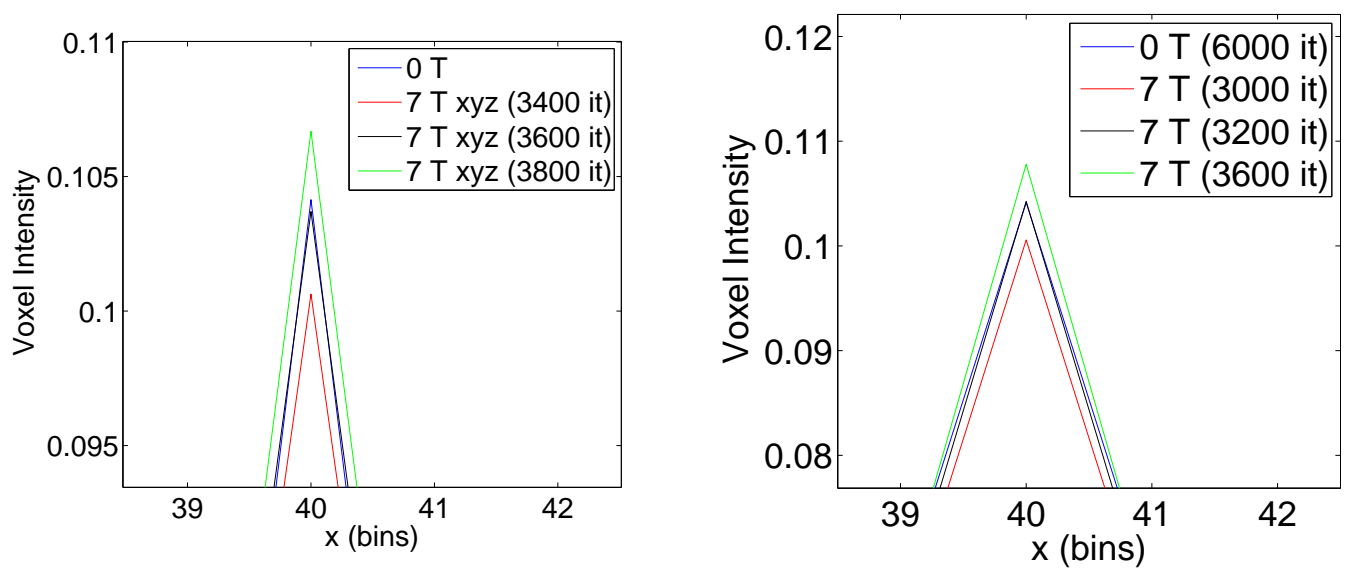

Figure 7.44: Zoomed in images on the peaks of the $\mathrm{x}$ projection from $7 \mathrm{~T}$ (3 orientations) and $7 \mathrm{~T}$ (axial) PSFs. The purpose of this plot is to show that 3600 and 3200 iterations (black line) of the 3D ML-EM positron range correction algorithm yields the closest match of the $7 \mathrm{~T}$ (3 orientation) PSF to the $0 \mathrm{~T}$ PSF. 

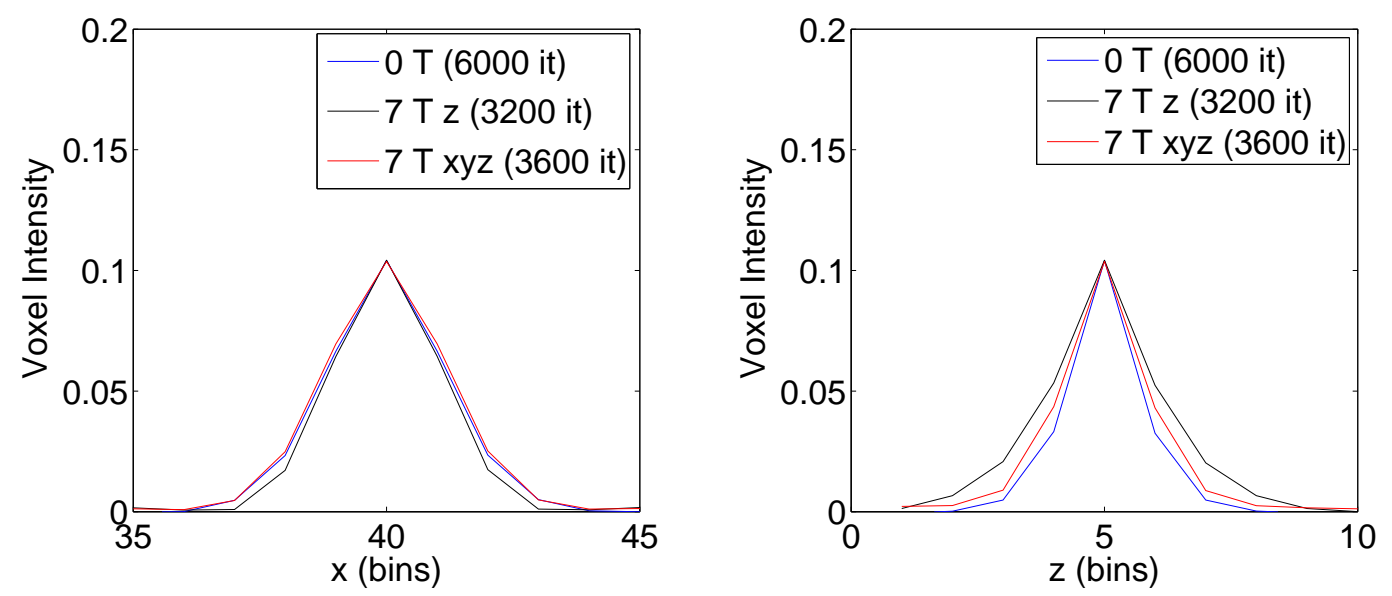

Figure 7.45: Projections through the maximum of the PSFs along the $\mathrm{x}$ and $\mathrm{z}$ directions of a 0 T PSF (iteration 6000) and a $7 \mathrm{~T}$ three B-field orientation PSF (iteration 3600) using the 3D ML-EM positron range correction algorithm. The difference between these two point spread functions is small, and can thus be used as a better estimate of the difference in the resolution-noise curves between the two data types. Table 7.2 contains the FWHM and FWTM values for the $\mathrm{x}$ and $\mathrm{z}$ projections.

\begin{tabular}{lccc} 
& $0 \mathrm{~T}$ & $7 \mathrm{~T}$ z $($ axial $)$ & $7 \mathrm{~T}$ xyz \\
\hline FWHM-X (mm) & $1.06 \pm 0.10$ & $1.01 \pm 0.10$ & $1.10 \pm 0.10$ \\
FWTM-X (mm) & $2.12 \pm 0.08$ & $1.96 \pm 0.08$ & $2.19 \pm 0.09$ \\
FWHM-Z (mm) & $0.61 \pm 0.09$ & $0.88 \pm 0.13$ & $0.68 \pm 0.10$ \\
FWTM-Z (mm) & $1.44 \pm 0.20$ & $2.22 \pm 0.31$ & $1.57 \pm 0.24$ \\
\hline
\end{tabular}

Table 7.2: Table of PSF of FWHM and FWTM in the $\mathrm{x}$ and $\mathrm{z}$ directions. 
PSFs along the xy plane and the xz plane, respectively. It should be noted that, depending on the quality of this printed document, it may be difficult to discern any differences in the PSFs. However, when viewed on a standard pdf viewer on a PC, the slight differences are more easily seen. From Fig. 7.47 it can be seen that the $0 \mathrm{~T}$ data is symmettric in the xy-plane, while the $7 \mathrm{~T}$ (3 orientation) data has a vague cross shaped pattern due to the constriction of the positron range along each applied magnetic field vector.

In order to better demonstrate the subtly differences between the PSFs, the difference between the $7 \mathrm{~T}$ (3 orientation) and the $0 \mathrm{~T}$ PSFs is shown on the left of Fig. 7.48. The maximum voxel intensity of the PSF is 0.1 , while the maximum differences in the voxel intensity are on the order of 0.003 (or a 3 percent difference). This plot is shown to illustrate that although the PSFs are very similar, that there is still some differences that can be corrected for in future work. The right image shows the difference between $7 \mathrm{~T}$ (axial) and the $0 \mathrm{~T}$ PSFs in the xy plane. Here the difference between the plots is more pronounced with a maximum six percent difference between the two PSFs' voxel intensities.

Figure 7.48 are images of the PSFs from the xz plane through the maximum of each PSF. Images of the difference between the $7 \mathrm{~T}$ (3 orientation) and $7 \mathrm{~T}$ (axial) PSF with the $0 \mathrm{~T}$ PSF are shown if Fig. 7.48. These images show that there is a larger difference between the $7 \mathrm{~T}$ PSFs and the $0 \mathrm{~T}$ PSF in the axial direction. The largest difference between individual voxel intensities is 10 percent for the $7 \mathrm{~T}$ (3 orientation) PSF, and up to 20 percent difference for the $7 \mathrm{~T}$ (axial) PSFs. A detailed study of the effects of the symmetry of the PSF on the resolved noise is left 

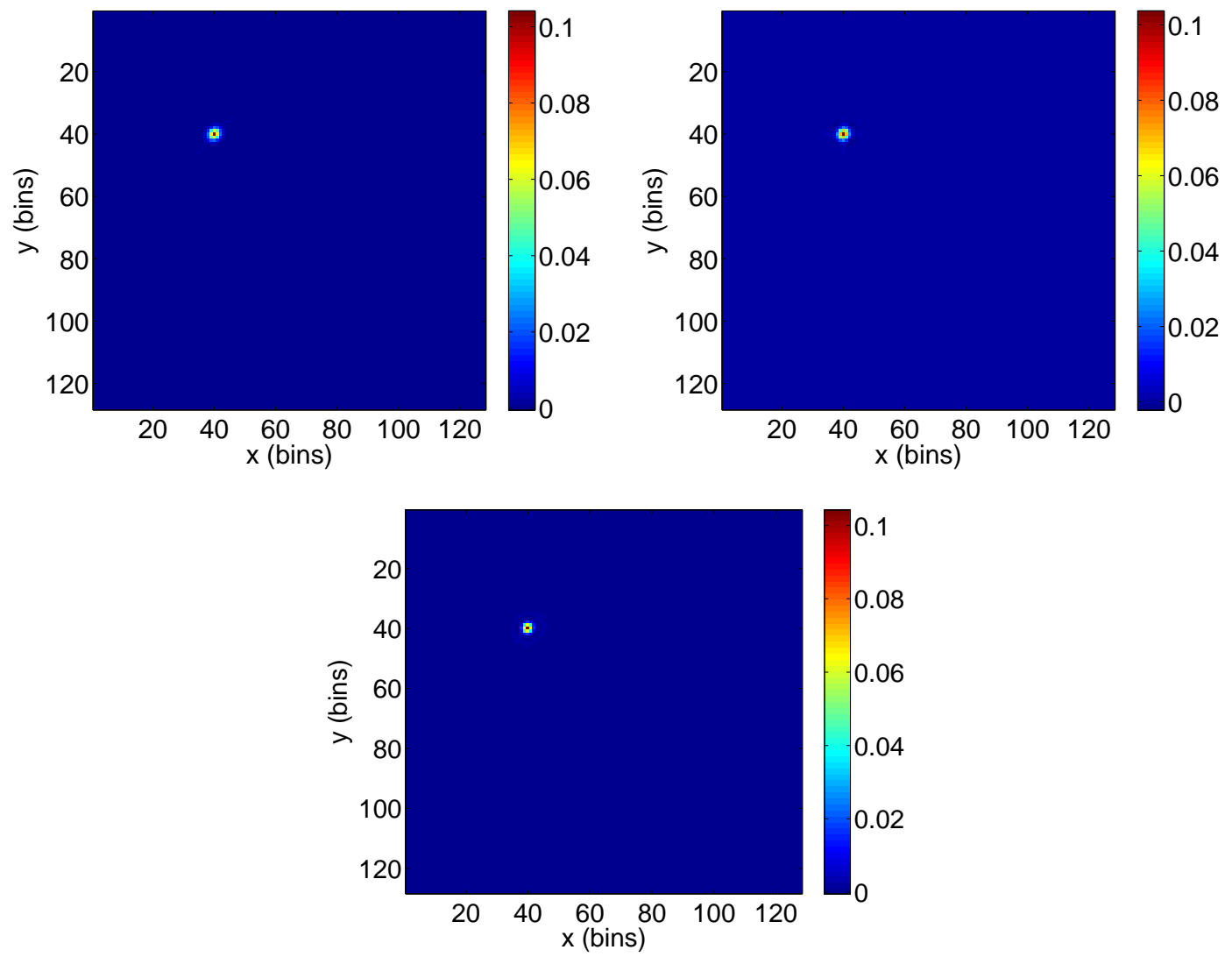

Figure 7.46: Reconstructed $0 \mathrm{~T}, 7 \mathrm{~T}$ (3 orientations), and $7 \mathrm{~T}$ (axial) PSFs reconstructed to iterations 6000,3600 , and 3200, respectively. The purpose of the plot is to shown that the difference discussed between the PSF is relatively small when compared to the entire FOV. 

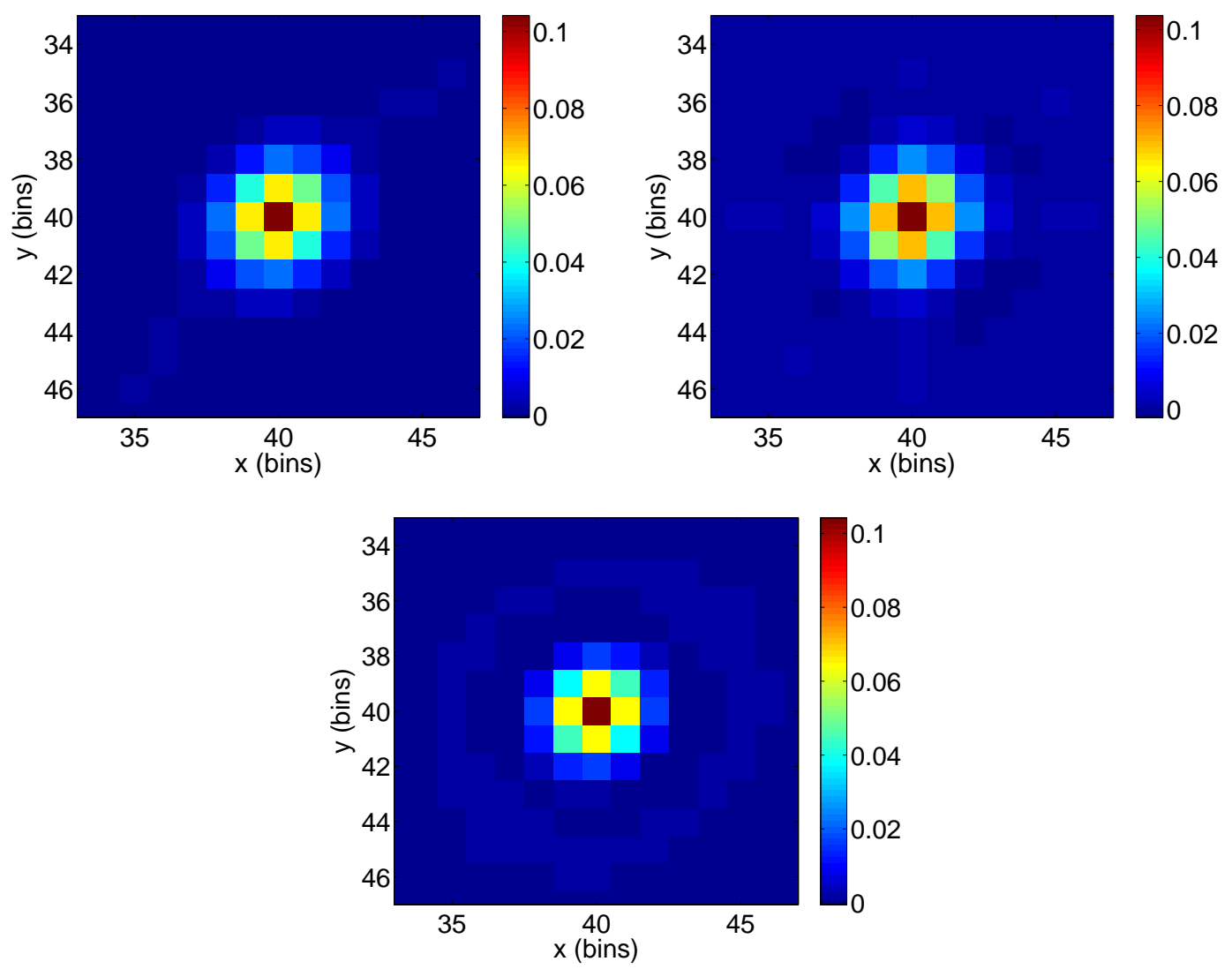

Figure 7.47: Zoomed in images of the reconstructed $0 \mathrm{~T}, 7 \mathrm{~T}$ (3 orientations), and $7 \mathrm{~T}$ (axial) PSFs reconstructed with iterations 6000, 3600, and 3200, respectively. 

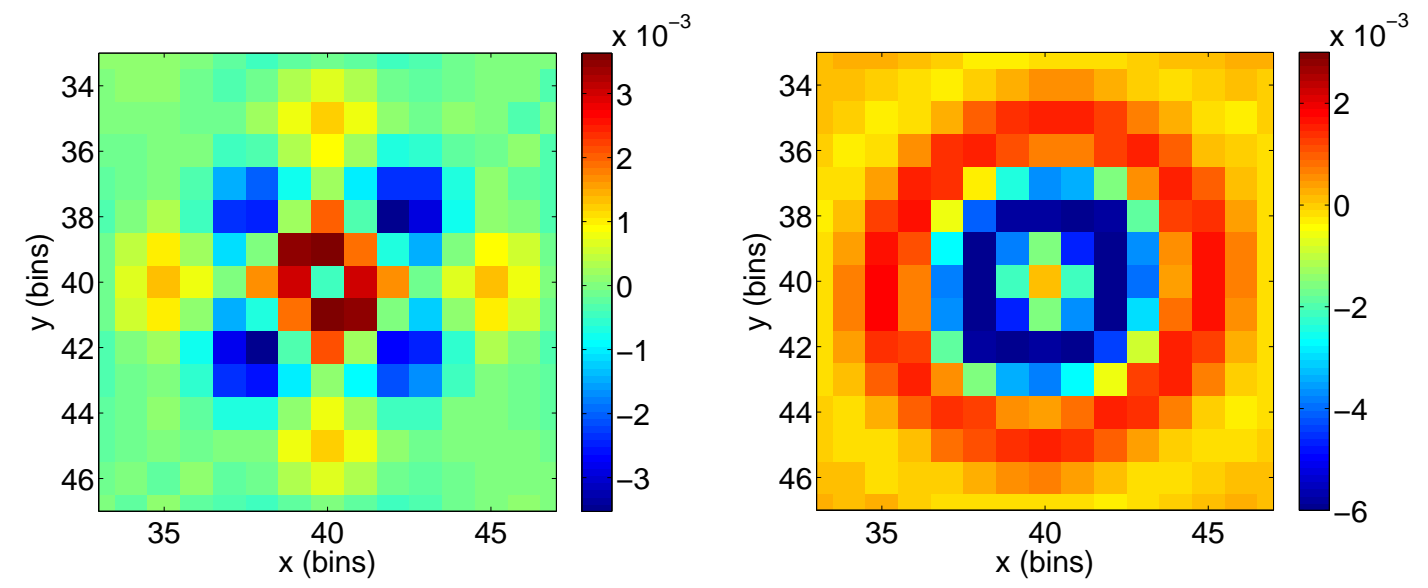

Figure 7.48: Left: the image of the difference between the $7 \mathrm{~T}$ (3 orientations) PSF and the 0 T PSF shown in Fig. 7.46. Right: the image of the difference between the $7 \mathrm{~T}$ (axial) PSF and the $0 \mathrm{~T}$ PSF shown in Fig. 7.46.

open for future studies. Ideally, the positron range can be completely corrected for, and thus there would be no differences in the PSFs.

Now that the number of iterations for each case that gives similar PSFs are known, the 25 realizations of the noisy data can be simulated and reconstructed for each case. Each 0 T noisy realization is reconstructed to 6000 iterations, each 7 T (3 orientation) noisy realization is reconstructed to 3600 iterations, and each $7 \mathrm{~T}$ (axial) noisy realization is reconstructed to 3200 iterations. Fig 7.51 are two example reconstructed noisy realizations using the $0 \mathrm{~T}^{68} \mathrm{Ga}$ positron range distribution in water, and the $7 \mathrm{~T}$ (3 orientation) ${ }^{68} \mathrm{Ga}$ positron range distribution in water. Twenty-five realizations are performed for each case and are used to create the final noise-resolution curve as outlined in the following section. 

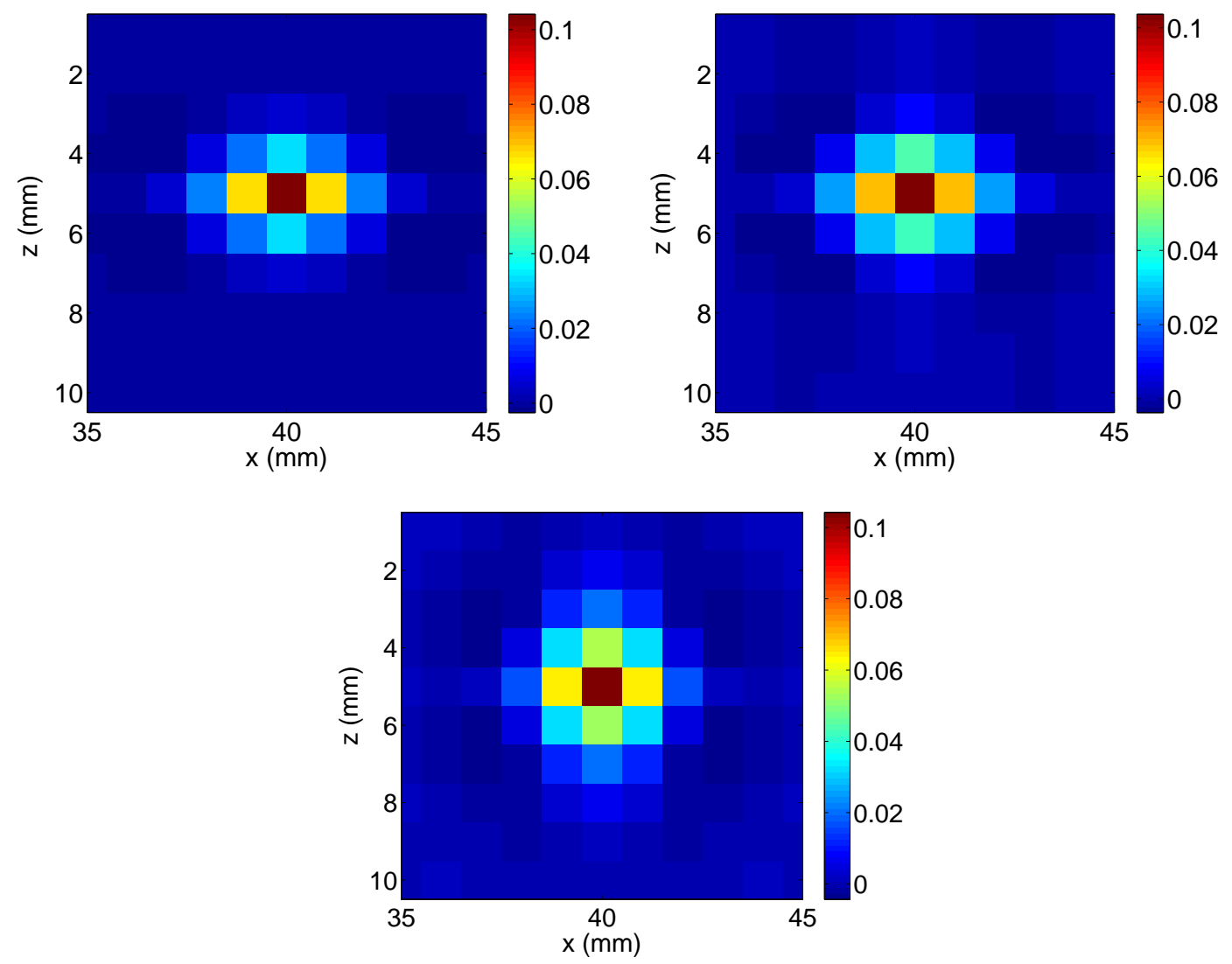

Figure 7.49: Difference plot for $0 \mathrm{~T}$ and $7 \mathrm{~T}$ small perturbative source in a constant background yz plane. The sum of the voxel content (in 3D) sums to 1.0 for both $0 \mathrm{~T}$ and $7 \mathrm{~T}$. Note that the positron range correction is having difficulty in this environment. In particular note the crossing artifact in the $7 \mathrm{~T}$ data suggesting that the algorithm is slow to remove the positron range. 

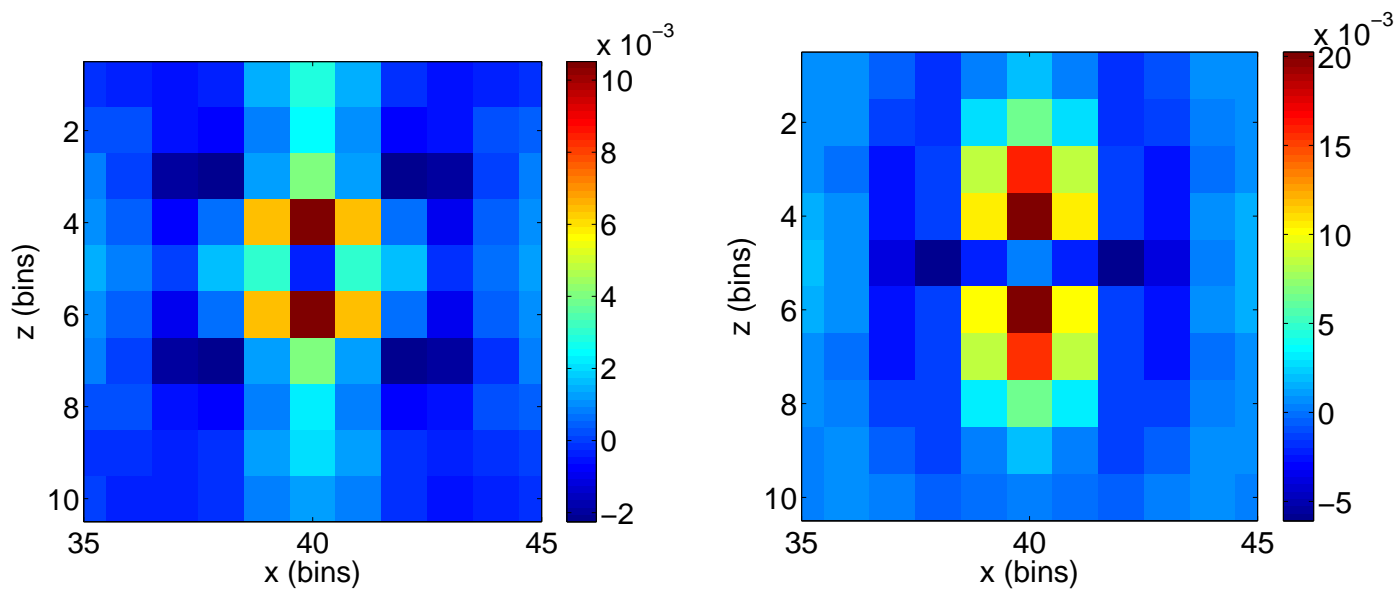

Figure 7.50: Left: the image of the difference between the $7 \mathrm{~T}$ (3 orientations) PSF and the 0 T PSF shown in Fig. 7.49. Right: the image of the difference between the $7 \mathrm{~T}$ (axial) PSF and the $0 \mathrm{~T}$ PSF shown in Fig. 7.46.
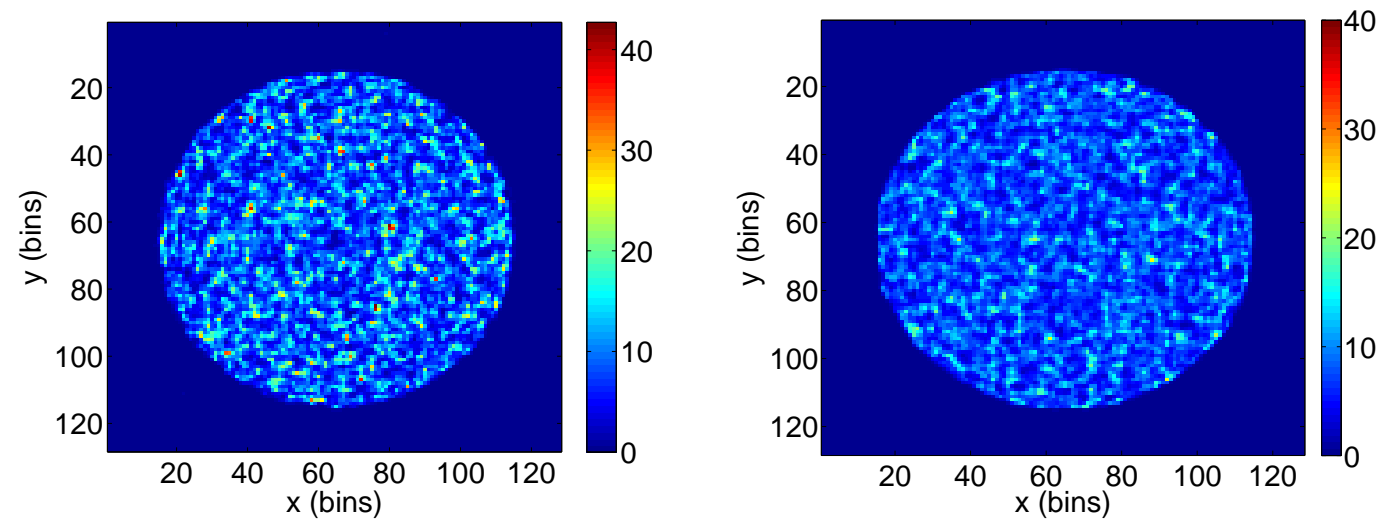

Figure 7.51: Images of the central slice of a uniform background using $10^{8}$ Poisson distributed events reconstructed for $0 \mathrm{~T}$ and $7 \mathrm{~T}$ data in three orientations. For the $0 \mathrm{~T}$ data 6000 iterations were used, and 3600 iterations for the $7 \mathrm{~T}$ (3 orientations) data. 


\subsubsection{Calculating the Noise-Resolution Curve}

This section explains the steps that are taken to create the noise-resolution curves once the PDF and the 25 noisy realizations have been reconstructed for each case. Each point on the graph is composed of two values (or coordinates), the resolution and the noise. Each of the resolution and noise coordinates are calculated separately. The two coordinates are paired by the Gaussian system resolution that blurs either the PSF or the noisy realizations. In other words, the resolution calculated from the blurred PSF and the noise calculated by the blurred set of noisy images constitute a paired (resolution, noise) coordinate.

As previously stated, in order to extract the resolution coordinates the PSF is blurred with the system resolution. The FWHM widths of the symmetric Gaussian system resolutions used in this study are $0.4 \mathrm{~mm}, 0.8 \mathrm{~mm}, 1.2 \mathrm{~mm}, 1.6 \mathrm{~mm}$, and $2.0 \mathrm{~mm}$. Figure 7.52 shows the projection along the $\mathrm{x}$-axis through the maximum of the 0 T PSF without any system resolution blurring and blurred for both system resolutions of $0.4 \mathrm{~mm}$ and $2.0 \mathrm{~mm}$. The resolution coordinate is the FWHM of each blurred PSF.

The noise value for each point is calculated by extracting the voxel intensity from voxel location (bin $\mathrm{x}=40$, bin $\mathrm{y}=40$, bin $\mathrm{z}=5$ which is the voxel that contained the perturbation that was used to created the PSF) for each of the convolutions of the 25 noisy realizations with the desired Gaussian system resolution. Figures 7.53 and 7.54 are bar graphs containing example voxel intensities of the 25 realizations used to calculate the standard deviation of the noise for the three cases addressed in this study. The voxel intensities in these plot were extracted from the 3D ML-EM positron range corrected images blurred with a $0.4 \mathrm{~mm}$ (Fig. 7.53) and a $2.0 \mathrm{~mm}$ 


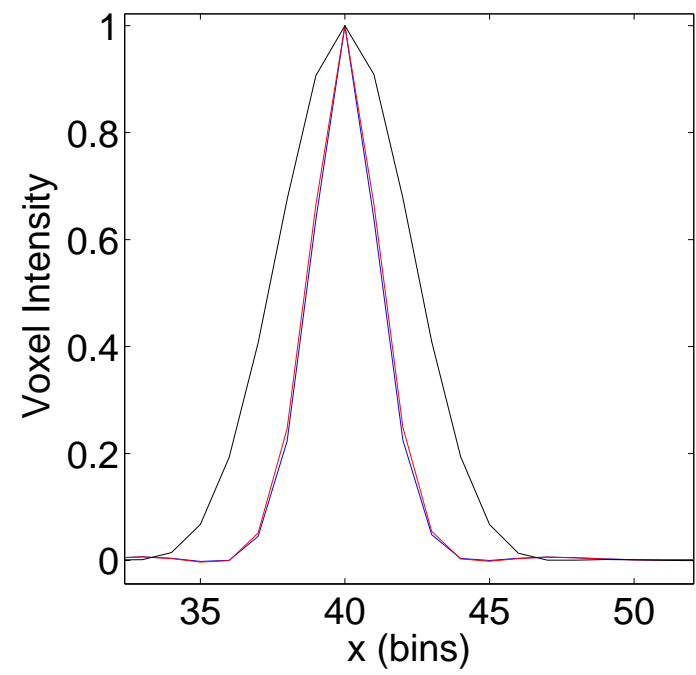

Figure 7.52: Example of the x-projection through the central maximum of the $0 \mathrm{~T}$ PSF without any system blurring (blue) and blurred with either a $0.4 \mathrm{~mm}$ (red) or $2.0 \mathrm{~mm}$ (black) FWHM 3D Gaussian.

(Fig. 7.54) FWHM Gaussian system resolution. The voxel location of the extracted values is the same voxel that contained the perturbative source for the portion of the algorithm that calculated the PSF such that any reconstruction artifacts will be present in both the PSF and in each noise realization. As expected, the variation in the noise decreases as the realizations are blurred with wider system resolutions.

The standard deviation is calculated for each blurred set of realizations using the following equation:

$$
\sigma=\sqrt{\frac{1}{N-1} \sum_{i=1}^{N}\left(x_{i}-\bar{x}\right)^{2}}
$$

where $x_{i}$ is the voxel intensity of the target bin for each realization and $\bar{x}$ is the mean value of the voxel intensities for $N$ realizations. The uncertainty in the calculated standard deviation of the noise can be calculated using the fractional uncertainty (this will give the size of the error bars in the noise-resolution plots). The fractional 


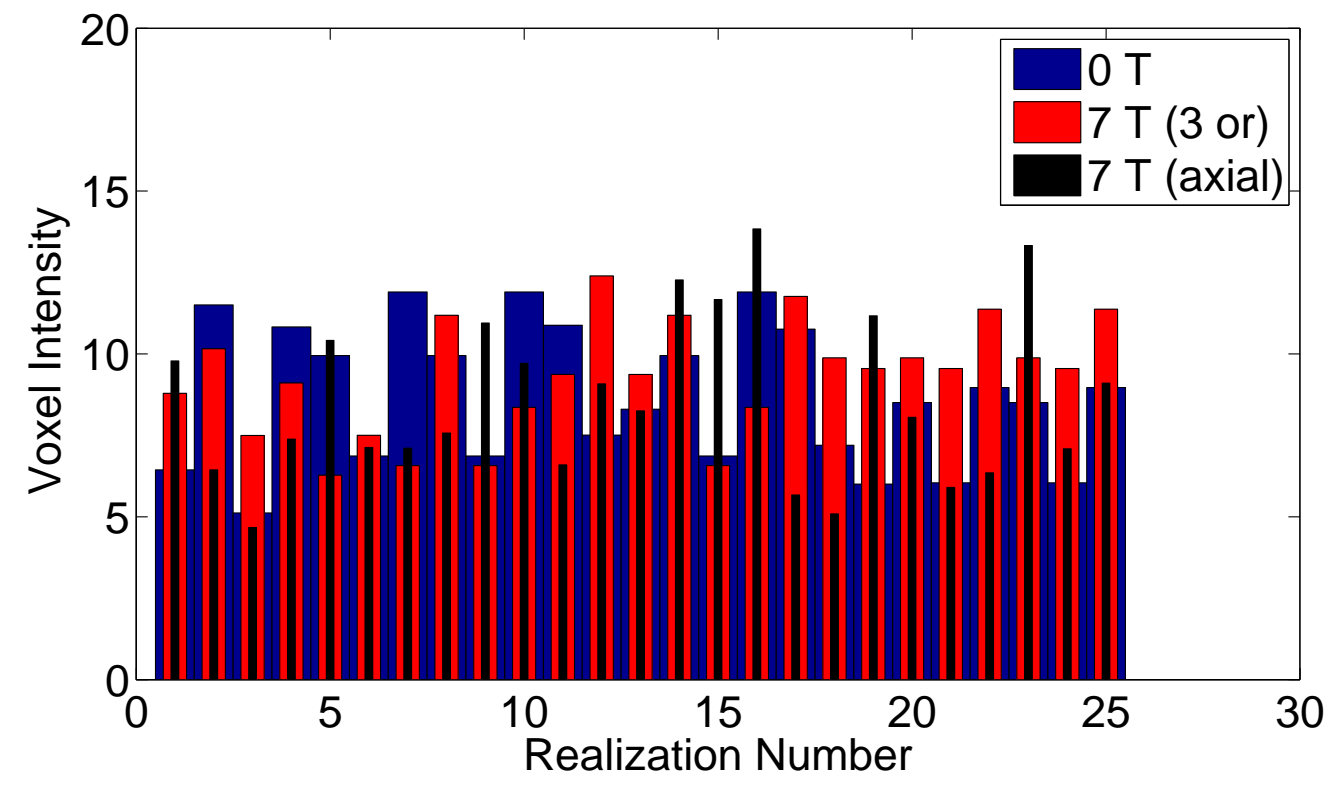

Figure 7.53: Bar graph of the voxel intensities blurred for a $0.4 \mathrm{~mm}$ FWHM Gaussian system resolution for the $0 \mathrm{~T}$ and $7 \mathrm{~T}$ (3 orientation) for $253 \mathrm{D}$ ML-EM reconstructions realizations used to calculate the first standard deviation in the noise value.

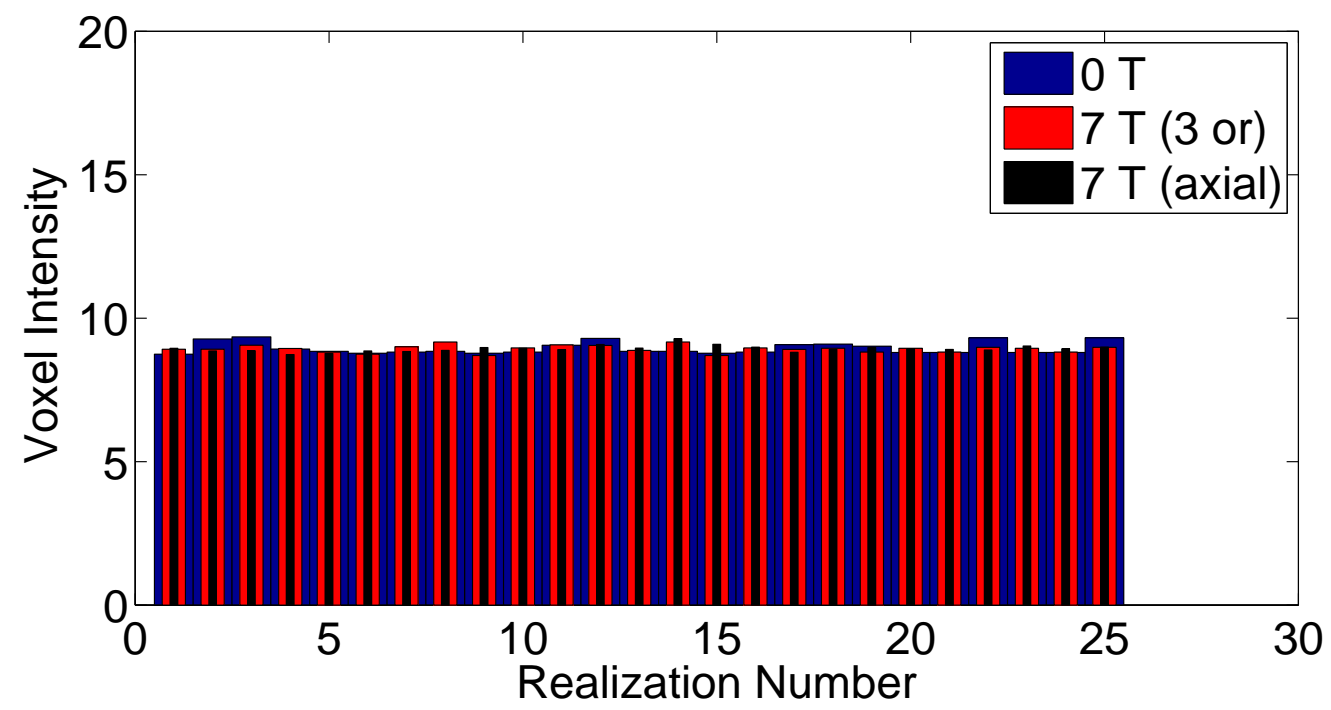

Figure 7.54: Bar graph of the voxel intensities blurred for a $2.0 \mathrm{~mm}$ FWHM Gaussian system resolution for the $0 \mathrm{~T}$ and $7 \mathrm{~T}$ (3 orientation) 3D ML-EM reconstructions realizations used to calculate the first standard deviation in the noise value. 
uncertainty is given by

$$
\delta=\frac{1}{\sqrt{2(N-1)}}
$$

where $N$ is the number of realizations (in this case $N=25$ ) [61]. For 25 realizations the fractional uncertainty in the standard deviation is 14.43 percent.

The results of the resolution noise study are shown in Figs. 7.55 and 7.56 where the resolution coordinates are from either the FWHM of the $\mathrm{x}$ or $\mathrm{z}$ blurred PSF.

Comparing the $0 \mathrm{~T}$ and $7 \mathrm{~T}$ (3 orientation) results one can see that there is a slight reduction in the standard deviation in the noise when operating in strong magnetic fields. This study has not addressed the uncertainty in the noise-resolution plots due to the wider width of the $7 \mathrm{~T}$ (3 orientation) PSF with respect to the $0 \mathrm{~T}$ PSF. The wider width will obscure some of the structure in the noise leading to artificially lower noise-resolution properties for the $7 \mathrm{~T}$ (3 orientation) curve. However, regardless of this slight uncertainty, it is clear from this plot that there is not a major advantage (or disadvantage) to collecting PET data with the magnetic field in three orientations for images reconstructing with a 3D ML-EM positron range correction algorithm.

Comparing the noise-resolution curves of the $7 \mathrm{~T}$ (axial) data along the $\mathrm{x}$ and $\mathrm{z}$ directions gives conflicting results. In the $\mathrm{x}$-direction, the $7 \mathrm{~T}$ (axial) case has the best noise-resolution properties; however, the $7 \mathrm{~T}$ (axial) system possess the poorest noise-resolution performance when viewed along the z-axis. These curves demonstrate the importance of having a uniform PSF. Depending on which FWHM value is used, the conclusion of the noise-resolution properties will differ. Also, recall that the $7 \mathrm{~T}$ axial PSF has a significantly wider PSF in the z-direction which will obscure some of the noise that would otherwise be revealed for a tighter PSF. This effect likely causes 


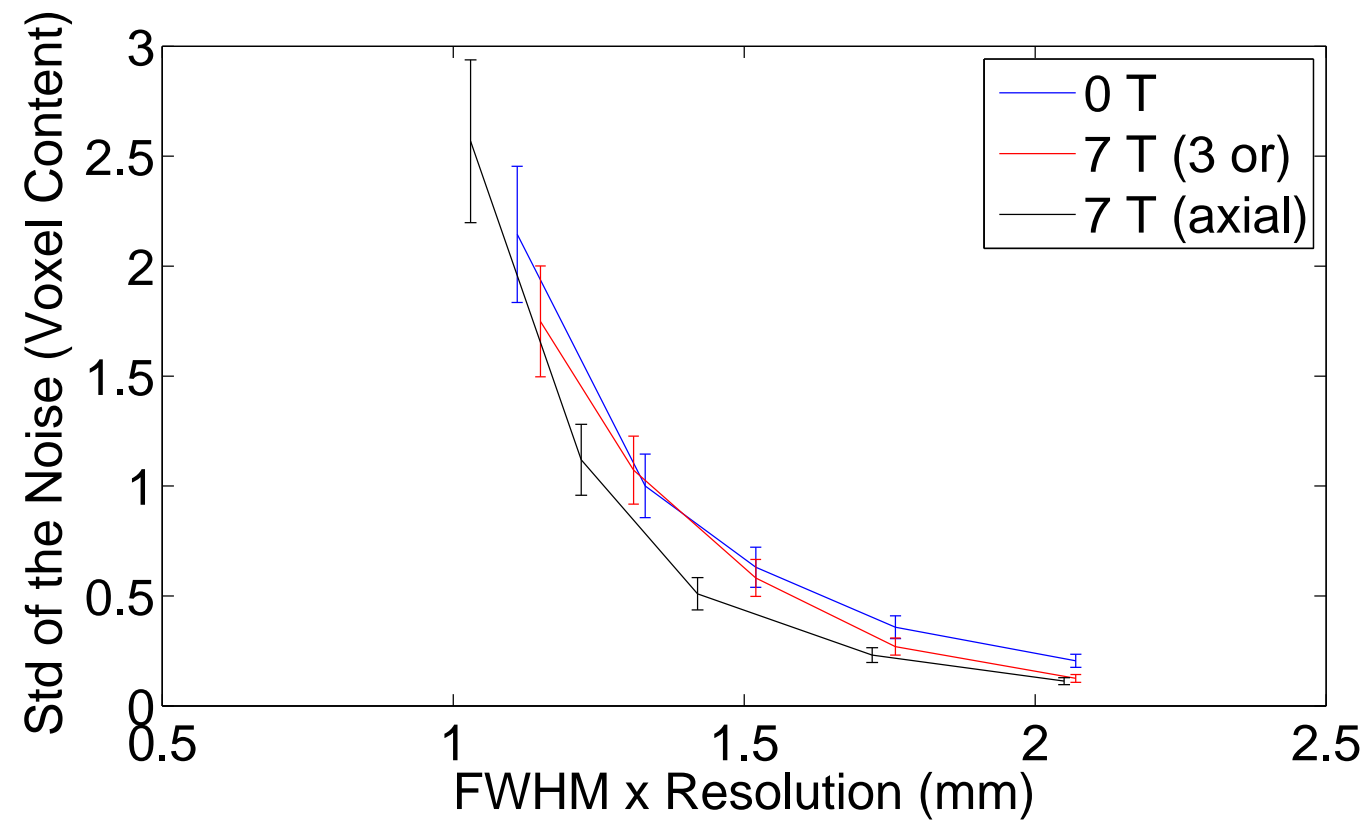

Figure 7.55: Plot of the standard deviation of the noise versus FWHM resolution in the x-direction. The error bars represent the uncertainty of the standard deviation. For 25 realization the uncertainty for every noise point is 14.43 percent.

the $7 \mathrm{~T}$ axial noise-resolution curve to be lower than it would be for a PSF that more resembles the $0 \mathrm{~T}$ or $7 \mathrm{~T}$ (3 orientations) PSFs.

Although it is not immediately apparent from the noise-resolution plots, there is an advantage to operating with a $7 \mathrm{~T}$ (3 orientation) for system possessing poorer resolutions. To see this, consider the relative noise advantage. The relative noise advantage is defined as the ratio of the standard deviation of the noise between the $0 \mathrm{~T}$ and $7 \mathrm{~T}$ (3 orientation)

$$
\text { Relative Noise Advantage }=\frac{\sigma_{0 T}}{\sigma_{7 T}} .
$$

A ratio of one means that there is no noise advantage to collecting data with three orthogonal orientations of a $7 \mathrm{~T}$ magnetic field. A ratio of less than one means that 


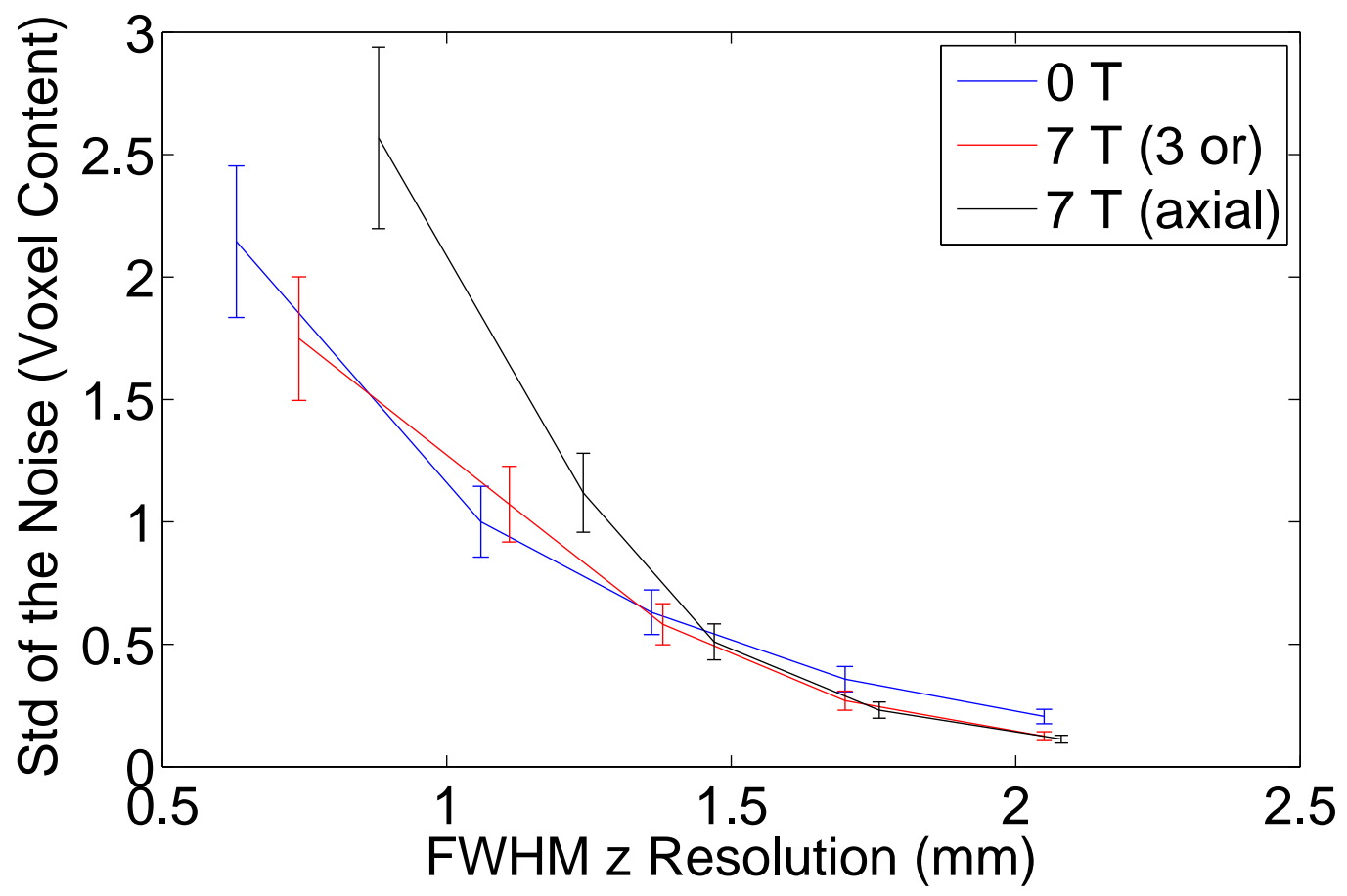

Figure 7.56: Plot of the standard deviation of the noise versus FWHM resolution in the z-direction. The error bars represent the uncertainty of the standard deviation. For 25 realization the uncertainty for every noise point is 14.43 percent. 
the $7 \mathrm{~T}$ (3 orientation) data has more noise at a given system resolution while a ratio greater than one means that there is less noise in the $7 \mathrm{~T}$ (3 orientation) data than in the $0 \mathrm{~T}$ data.

The ratio between the standard deviation in the noise at $0 \mathrm{~T}$ and $7 \mathrm{~T}$ (3 orientations), $\sigma_{0 T} / \sigma_{7 T}$, is plotted versus the width of the PSF in Fig. 7.57. The fractional uncertainty $\delta_{f}$ in the ratio is calculated using the standard uncertainty of quotients with known fractional uncertainties [61]:

$$
\delta_{f}=\frac{\sigma_{0 T}}{\sigma_{7 T}}\left[\frac{\delta_{0 T}}{\sigma_{0 T}}+\frac{\delta_{7 T}}{\sigma_{7 T}}\right]
$$

where $\delta_{0 T}$ and $\delta_{7 T}$ are the uncertainties of the standard deviation (calculated using eq. 7.2). Figure 7.57 shows that although there is little to no advantage in collecting data in three magnetic field orientations with respect to the data collected for high resolution systems, that there is a factor of two benefit in collecting data in three magnetic field orientations for poor resolution systems on the order of $2.5 \mathrm{~mm}$ FWHM. It should be noted that a $2.5 \mathrm{~mm}$ FWHM resolution is unacceptably large for small animal PET scanners; however, this is actually a fairly high resolution for full body human scanners. Viewed in this light there is a major noise advantage to collecting data in multiple orientations for human full body scans. This result is very interesting; however, the implementation of rotating a body into three orthogonal orientations within an MRI scanner is not feasible due to the relative rigidity of the human body.

\subsection{Conclusions and Future Work}

This work has addressed the question: how do magnetic fields affect the image quality of high resolution PET systems? This question has been addressed using both Monte Carlo simulation methods and experimental measurements. 


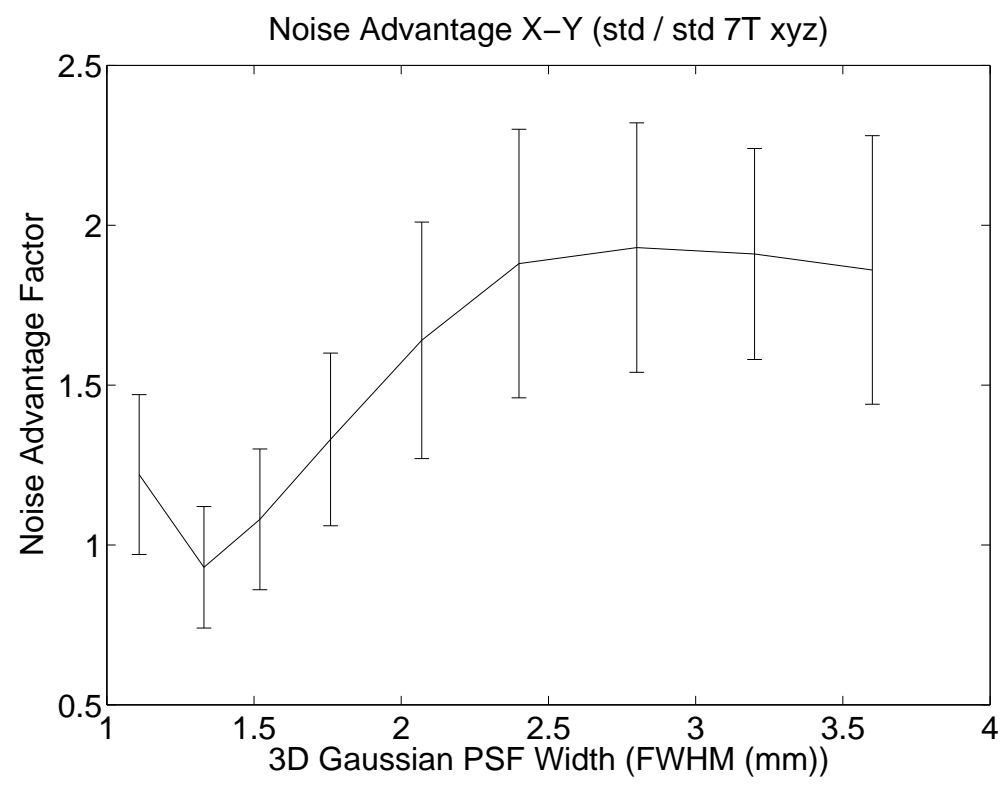

Figure 7.57: Relative advantage

The simulations were performed using the EGS4 Monte Carlo code and gave results for the positron range in water that are consist with the literature in the absence of a magnetic field. The flight path of the positron as it penetrated matter in the presence of a magnetic field was modeled in the user written HOWFAR routine using the UCBEND subroutine. The EGS4 simulations suggested that the tails of the positron-electron annihilation distributions for high energy positron emitters can be greatly reduced by the application of a strong magnetic field directed transverse to the image plane. Simulation results for the reduction of the positron range in lung tissue were especially impressive. Although, a study of the improvement in lung tissue in strong magnetic fields was not a focus of this study, this effect deserves further study and will likely lead to larger relative improvements in the image quality in the presence of magnetic fields than in water due to the much larger positron spread at 
$0 \mathrm{~T}$ as compared to $7 \mathrm{~T}$. A full EGS4 simulation was performed that described the geometry of the experimental small animal PET prototype such that the full data could be simulated to compare to the experimental results.

This work also demonstrates that with only a slight modification silicon detectors can operate without any change in performance within magnetic fields up to $8 \mathrm{~T}$. The silicon detectors used in this study are composed of 512 pads $(1.4 \mathrm{~mm} \times 1.4 \mathrm{~mm}$ in area and $1.0 \mathrm{~mm}$ thick) readout by four VATAGP3 front-end ASICs. A small animal PET test bench was constructed with two such silicon detectors positioned edge on, defining a $1.0 \mathrm{~mm}$ thick detection plane. A pneumatic pump that can operate in strong magnetic fields was used to rotate the source distribution to various angular positions in order to collect data with full angular sampling.

The small animal PET test bench was used to measure the image resolution of low and high energy positron emitters, ${ }^{22} \mathrm{Na}$ and ${ }^{68} \mathrm{Ga}$, respectively. Both the ML-EM reconstructions (without positron range corrections) between the measured experimental data and the EGS4 simulated data are in good agreement. It has also been demonstrated, both through measurements and simulations, that the artifacts from high kinetic energy positron sources positioned off-plane lead to more defined artifacts when a $7 \mathrm{~T}$ magnetic field is applied in the axial direction than in the absence of a magnetic field. This is due to the constriction of the positron range in the direction transverse to the applied magnetic field vector. However, this effect is likely more severe when using a stack of $2 \mathrm{D}$ PET images to create a 3D image in contrast to images created from fully 3D PET systems (see section 6.5).

In this work the initial results and characteristics of a 3D ML-EM positron range correction algorithm are explored. This reconstruction algorithm was used to show 
that the positron range can be completely corrected for when reconstructing simple sources at both $0 \mathrm{~T}$ and in strong magnetic fields. Qualitative comparisons of simulated phantom images reconstructed with the 3D ML-EM positron range correction algorithm suggest a slight improvement in the image quality using three orientations of a $7 \mathrm{~T}$ magnetic field simultaneously to reconstruct the images in comparison to correcting for only the $0 \mathrm{~T}$ positron range. For all three cases the 3D ML-EM positron range correction algorithm performed well. These result show that artifacts from high energy positron emitters can be statistically corrected for in PET/MRI systems using a 3D ML-EM correction algorithm to reconstruct the data.

A study of the noise versus resolution was performed to evaluate the relative advantages/disadvantages of collecting data for three cases (see Fig. 7.1) when using the 3D ML-EM positron range correction algorithm. In this study it was found that in the presence of a background source the images reconstructed with the 3D ML-EM positron range correction algorithm converge slowly and yield asymmetric PSFs that can be difficult to compare depending on the number of magnetic field orientations used to reconstruct the data. In particular the 3D ML-EM positron range correction algorithm could not yield a PSF for the $7 \mathrm{~T}$ (axial) study that closely matched both the $0 \mathrm{~T}$ and $7 \mathrm{~T}$ (3 orientation) PSFs. As a result, the $7 \mathrm{~T}$ (axial) noise-resolution curve could not be compared to the $0 \mathrm{~T}$ and $7 \mathrm{~T}$ (3 orientation) resolution-noise curve. This study has demonstrated that there is neither an obvious advantage (or disadvantage) to collecting data at $0 \mathrm{~T}$ compared to $7 \mathrm{~T}$ (3 orientations) when a 3D ML-EM positron range algorithm is used (see Figs. 7.55 and 7.56) (these results may differ for fully 3D PET systems). Although the noise-resolution curves are very 
similar, there is a factor of two improvement in the noise at poorer resolutions as can be seen by plotting the relative noise advantage curve (see Fig. 7.57).

It should be noted that reconstructing images using additional magnetic field orientations (more than three) may yield improved noise-resolution properties. This study is left open for future research; however, the implementation of building a device that can collect data in many magnetic field orientations in the confined space of an MRI will present significant engineering hurtles.

In conclusion, the image blur due to the range of the positron can be reduced in the plane transverse to a strong magnetic field; however, artifacts aligned with the magnetic field vector can arise due to high kinetic energy positron emitters located off-plane ${ }^{8}$. It has been demonstrated that these artifacts can be statistically corrected for by using a 3D ML-EM positron range correction algorithm. This result has direct implications for PET/MRI systems attempting to image high kinetic energy positron emitters. Finally, it has been shown that there is not a major advantage to collecting data in three orthogonal magnetic field orientations relative to data collected at $0 \mathrm{~T}$ for high resolution systems (on the order of a $1.0 \mathrm{~mm}$ FWHM). However, for poorer resolution systems $(2.5 \mathrm{~mm} \mathrm{FWHM})$ there is a factor of two reduction in the noise using data collected with three orthogonal $7 \mathrm{~T}$ magnetic field orientations in comparison to data collected at $0 \mathrm{~T}$.

\footnotetext{
${ }^{8}$ These artifacts are likely to be more severe for PET systems using a stack of 2D planar PET images to create a 3D image than in comparison to a fully 3D PET system
} 


\section{BIBLIOGRAPHY}

[1] M. N. W. EDITOR and J. N. A. EDITOR, Emission Tomography: The Fundamentals of PET and SPECT. Elsevier Academic Press, 525 B Street, Suite 1900, San Diego, California 92101-4495, USA, 2004.

[2] W. W. Moses, "Recent advances and future advances in time-of-flight PET," Nucl. Inst. Meth. Phys. Res. A 580 (October, 2007) 919-924.

[3] C. S. Levin, "Design of a high-resolution and high-sensitivity scintillation crystal array for PET with nearly complete light collection," IEEE Transactions on Nuclear Science 49 (October, 2002) 2236-2243.

[4] J. R. Stickel and S. R. Cherry, "High-resolution PET detector design: modeling components of intrinsic spatial resolution," Physics in Medicine and Biology $\mathbf{5 0}$ (2005) 179-195.

[5] C. S. Levin, "New imaging technologies to enhance the molecular sensitivity of positron emission tomography," Preceedings of the IEEE 96 (March, 2008) 439-467.

[6] G. F. Knoll, Radiation Detection and Measurement Third Edition. John Wiley and Sons Inc, 2000.

[7] S. Park, W. Rogers, S. Huh, H. Kagan, K. Honscheid, D. Burdette, E. Chesi, C. Lacasta, G. Llosa, M. Mikuz, A. Studen, P. Weilhammer, and N. Clinthorne, "A prototype of very high-resolution small animal PET scanner using silicon pad detectors," Nucl. Instr. and Meth. A 570 (2007) 543-555.

[8] A. Studen, D. Burdette, E. Chesi, N. Clinthorne, S. Huh, K. Honscheid, H. Kagan, C. Lacasta, G. Llosa, M. Mikuz, W. Rogers, and P. Weilhammer, "Timing in thick silicon pad detectors," IEEE Nucl. Sci. Symp. Conf. Record 8 (2006) 1087-1090.

[9] G. C. Giakos, S. Vedantham, S. Chowdhury, J. Odogba, A. Dasgupta, R. Guntupalli, S. Suryanarayanan, V. Vega-Lozada, M. Sridhar, M. Khyati, and N. Shah, "Timing characteristics of a $c d_{1-x} z n_{x}$ te detector-based x-ray imaging system," IEEE Transactions on Instrumentation and Measurement 48 (October, 1999) 909-914. 
[10] D. Burdette and et al., "A study of the effects of strong magnetic fields on the image resolution of PET scanners," IEEE Conference Records (2007).

[11] K. Shibuya, D. Yoshida, F. Nishikido, T. Suzuki, T. Tsuda, N. Inadama, and T. Yamaya, "Annihilation photon acollinearity in PET: volunteer and phantom FDG studies," Phys. Med. Biol. 52 (2007) 5249-5261.

[12] E. M. Rohren, T. G. Turkington, and E. Coleman, "Clinical applications of PET in oncology," Radiology 231 (2004) 305-332.

[13] Y. Tai and P. Piccini, "Applications of positron emission tomography PET in neurology," J Neurol Neurosurg Psychiatry 75 (May, 2004) 669-676.

[14] F. M. Bengel, "Positron emission tomography and magnetic resonance imaging in heart failure," Journal of Nuclear Cardiology 13 (March/April, 2006).

[15] R. Myers, "The biological application of small animal PET imaging," Nuclear Medicine and Biology 28 (2001) 585-593.

[16] M. Larobina, A. Brunetti, and M. Salvatore, "Small animal PET: A review of commercially available imaging systems," Curr. Med. Imag. Rev., 2 (2006) 187-192.

[17] K. C. Schmidt and C. B. Smith, "Resolution, sensitivity and precision with autoradiography and small animal positron emission tomography: implications for functional brain imaging in animal research," Nuclear Medicine and Biology 32 (2005) 719-725.

[18] T. Budinger, D. Beneron, and A. Koretsky, "Imaging transgenic animals," Annu. Rev. Biomed Eng. 1 (1999) 611-648.

[19] A. F. Chatziioannou, "PET scanners dedicated to molecular imaging of small animal models," Molecular Imaging and Biology 4 (2002), no. 1, 47-63.

[20] R. Raylmann, B. Hammer, and N. Christensen, "Combined MRI-PET scanner: A Monte Carlo evaluation of the improvements in PET resolution due to the effects of a static homogeneous magnetic field," IEEE Trans. Nucl. Sci. 43 (1996) 2406-2412.

[21] W. Nelson, H. Hirayama, and D. Rogers, The EGS4 Code System. Stanford Linear Accelerator Center Report SLAC-265, Stanford, CA, 1985.

[22] A. F. Bielajew and D. W. O. Rogers, "PRESTA: The parameter reduced electron-step transport algorithm for the electron Monte Carlo transport," Nucl. Instr. and Meth. B 18 (1987) 165-181.

[23] C. Wu and S. Moskowski, Beta Decay. Interscience, New York, 1966. 
[24] C. Levin and E. Hoffman, "Calculation of positron range and its effect on the fundamental limit of positron emission tomography system spatial resolution," Phys. Med. Biol. 44 (1999) 781-799.

[25] H. Bethe, "Moliere's theory of multiple scattering," Phys. Rev. 89 (1953) $1256-1266$.

[26] H. Koch and J. Motz, "Bremsstrahlung cross-section formulas and related data," Rev. Mod. Phy. 31 (1959) 920-955.

[27] R. Sternheimer, "The density effect for the ionization loss in various materials," Phyical Review 88 (1952), no. 4, 851-859.

[28] R. Sternheimer, "Density effect for ionization loss of charged particles," Physical Review 145 (1966), no. 1, 247-145.

[29] M. Berger and S. M. Seltzer, "Table of energy losses and ranges of electrons and positrons," report number nasa-sp-3012, National Aeronautics and Space Administration, 1964.

[30] K. Kase and W. Nelson, Concepts of Radiation Dosimetry. Pergamon Press, New York, 1979.

[31] A. Sánchez-Crespo, P. Andreo, and S. A. Larsson, "Positron flight in human tissues and its influence on PET image spatial resolution," Eur. J. Nucl. Med. Mol. Imaging 31 (2004), no. 1, 44-51.

[32] C. Champion and C. L. Loirec, "Positron follow-up in liquid water: II. spatial and energetic study for the most important radioisotopes used in PET," Phys. Med. Biol. 52 (2007) 6605-6625.

[33] G. Bertolini and A. Coche, Semiconductor Detectors. John Wiley and Sons, Inc., 1968. 4.

[34] A. Studen, Compton Camera with Position Sensitive Silicon Detectors. PhD thesis, University of Ljubljana, 2005.

[35] N. Clinthorne, S. Park, W. Rogers, P. Chiao, and the CIMA collaboration, "Multi-resolution image reconstruction for a high-resolution small animal PET device," IEEE Nucl. Sci. Symp. Conf. Record 6 (2004) 1997-2001.

[36] A. Studen, D. Burdette, E. Chesi, V. Cindro, N. Clinthorne, W. Dulinski, J. Fuster, L. Han, H. Kagan, C. Lacasta, G. Llosa, A. Marques, N. Malakhov, D. Meier, M. Mikuz, S. Park, S. Roe, W. Rogers, J. Steinberg, P. Weilhammer, S. Wilderman, L. Zhang, and D. Zontar, "First coincidences in pre-clinical compton camera prototype for medical imaging," Nucl. Instr. and Meth. A 531 (2004) 258-264. 
[37] C. S. Levin and H. Zaidi, "Current trends in preclinical PET system design," PET Clin 2 (2007) 125-160.

[38] C. Jacoboni, C. Canali, G. Ottaviani, and A. Quaranta, "A review of some charge transport properties of silicon," Solid-State Electron. 20 (1977) 77-89.

[39] C. A. Klein, "Semiconductor particle detectors: A reassessment of the Fano factor situation," IEEE Trans. Nucl. Sci. 15 (1968) 214-225.

[40] U. Fano, "Ionization yield of radiations. II. the fluctuations of the number of ions," Phys. Rev. 72 (1947), no. 1, 26-29.

[41] G. D. Geronimo, P. O'Connor, V. Radeka, and B. Yu, "Front-end electronics of imaging detectors," Pres. Imaging 2000 Conference (2000).

[42] H. Kagan, J. Alexander, A. Bean, C. Bebek, G. Brandenburg, C. Darling, J. Duboscq, J. Fast, A. Foland, K. Gan, P. Hopman, R. Kass, P. Kim, N. Menon, D. Miller, B. Nemati, J. Oliver, C. Rush, I. Shipsey, P. Skubic, M. Spencer, C. Uhl., C. Ward, R. Wilson, M. Yurko, and M.M.Zoeller, "Low noise electronics for the CLEO III silicon detector," Nucl. Inst. Meth. A 383 (1996) 189-192.

[43] SINTEF, "Foundation of Scientific and Industrial Research at the Norwegian Institute of Technology, Electronics and Cybernetics, microsystems." http://www.oslo.sintef.no/ecy/. Oslo and Trondheim, Norway.

[44] S.-J. Park, A Very High Resolution Small Animal PET Based on the Compton PET Concept. PhD thesis, The University of Michigan, 2005.

[45] M. Hirasawa and T. Tomitani, "Effect of compensation for scattering angular uncertainty in analytical compton camera reconstruction," Physics in Medicine and Biology 49 (2004) 2083-2093.

[46] J. Gaboian, "A survey of common-mode noise," Report SKKA057, Texas Instruments, 1999.

[47] C. Lacasta, E. Cochran, K. Honscheid, G. Llosa, and A. Student, "DAQ++: A c++ data acquisition software framework," IEEE Conference Records (2007).

[48] A. C. Kak and M. Slaney, Principles of Computerized Tomographic Imaging. SIAM, 2001.

[49] A. Dempster, N. Laird, and D. Rubin, "Maximum likelihood from incomplete data via the EM algorithm," Journal of the Royal Statistical Society 39 (1977), no. 1, 1-38.

[50] L. Shepp and Y. Vardi, "Maximum likelihood reconstruction for emission tomography," Trans. on Medical Imaging MI-1 (October, 1982) 113-122. 
[51] W. I. Zangwill, Nonlinear Programming. Prentice-Hall, Englewoods Cliffs, NJ, 1969.

[52] D. J. Horen, "Decay of the radioisotopes Ge $\mathrm{Ge}^{68}$ and Ga ${ }^{68}$," Phys. Rev. 113 (January, 1959) 572-578.

[53] "Eckert and Ziegler Isotope Products." http://www.analyticsinc.com/.

[54] W. Moses, P. Virador, S. Derenzo, R. Huesman, and T. Budinger, "Design of a high-resolution, high-sensitivity PET camera for human brains and small animals," IEEE Transactions on Nuclear Science 44 (August, 1997) 1487-1491.

[55] B. E. Hammer, N. L. Christensen, and B. G. Heil, "Use of a magnetic field to increase the spatial resolution of positron emission tomography," Med. Phys. 21 (December, 1994) 1917-1920.

[56] B. E. Hammer and N. L. Christensen, "Measurement of positron range in matter in strong magnetic fields," IEEE Trans. Nucl. Sci. 42 (August, 1995) $1371-1376$.

[57] J. Gaa, E. J. Rummeny, and M. Seemann, "Whole-body imaging with PET/MRI," Eur. J. Med. Res. 9 (2004) 309-312.

[58] R. Jaszczak, J. Li, H. Wang, M. Zalutsky, and E. Coleman, "Pinhole collimation for ultra-high-resolution, small-field-of-view SPECT," Phys. Med. Biol. 39 (1994) 425-437.

[59] C. Knoess, S. Siegel, A. Smith, D. Newport, N. Richerzhagen, A. Winkeler, A. Jacobs, R. Goble, R. Graf, K. Wienhard, and W. Heiss, "Performance evaluation of the micropet R4 pet scanner for rodents.," Eur J Nucl Med Mol Imaging 5 (2003) 737-47.

[60] J. A. Fessler and W. L. Rogers, "Resolution properties of regularized image reconstruction methods," Technical Report 297, The University of Michigan, Dept. of Electrical Engineering and Computer Science, 1997.

[61] J. R. Taylor, An Introduction to Error Analysis: The Study of Uncertainties in Physical Measurements. University Science Books, 55D Gate Five Road Sausalito, CA 94965, 2 ed., 1997. 